\title{
BOUNDARY LAYER TRANSITION IN ATTACHED AND SEPARATED FLOWS AT LOW REYNOLDS NUMBERS
}

\author{
by \\ Stephen K. Roberts \\ B.Eng., M.A.Sc. (Carleton University)
}

SUBMITTED IN PARTIAL FULFILLMENT OF THE

REQUIREMENTS FOR THE DEGREE OF

DOCTOR OF PHILOSOPHY IN AEROSPACE ENGINEERING

AT

CARLETON UNIVERSITY

OTTAWA-CARLETON INSTITUTE OF MECHANICAL AND AEROSPACE ENGINEERING OTTAWA, ONTARIO, CANADA

MAY 24, 2005

(C) Copyright by Stephen K. Roberts, 2005 


\section{Abstract}

The transition of a boundary layer from a laminar to a turbulent state is known to be affected by several flow and surface conditions, including flow Reynolds number, streamwise pressure gradient, free-stream turbulence, surface roughness, and periodic oscillations in free-stream velocity. Although the effects of these parameters on the transition process have been the subject of numerous investigations over the past decades, the interactions between these conditions, when present simultaneously, have yet to be fully documented. The current research program was undertaken to increase our understanding of the process of boundary layer transition, and to quantify the relative importance of these conditions.

The majority of the present study consists of hot-wire anemometer measurements of a two-dimensional boundary layer developing over a flat surface. The ranges of Reynolds number, pressure distribution, free-stream turbulence, periodicunsteadiness, and surface roughness were representative of the suction side of low pressure turbine blades, and resulted in both attached-flow and separation-bubble transition processes. Based on the experimental results, an improved transition model to predict these effects is presented. This model is applicable to a broader range of flow and surface conditions than available alternatives.

The experiments are complemented by numerical simulation of one of the test cases, by means of large-eddy simulation. The simulation provides an opportunity for detailed study of the unsteady flow phenomena and vortex shedding processes that accompany transition and reattachment of the separation bubble. 


\section{Acknowledgements}

I would first and foremost like to thank my thesis supervisor, Prof. Metin Yaras, for his enthusiastic participation and continuous support during this research. I consider myself very fortunate that many thankless tasks, such as the majority of design and commissioning of the experimental test section, had already been completed before I joined this project.

I am also very grateful for the cooperation of Profs. J. Dey (Indian Institute of Science, Bangalore, India), J.P. Gostelow (University of Leicester, UK), and R.J. Volino (U.S. Naval Academy, Annapolis, MD) for providing additional experimental data complementing that of the current study.

The assistance that has been provided over the course of my studies by the many members of the administrative and technical staff at the Department of Mechanical and Aerospace Engineering is greatly appreciated.

The financial support of Pratt \& Whitney Canada and of the Natural Science and Engineering Research Council (NSERC) is gratefully acknowledged.

I am especially grateful for the support I have received over the years from my family and friends. Finally, this achievement would not have been possible without the patience and support of my partner, Carol Weller.

Stephen K. Roberts

Ottawa, Ontario

May 24, 2005 


\section{Contents}

Abstract iii

Acknowledgements iv

Figures $\quad$ ix

Tables $\quad$ xiv

$\begin{array}{ll}\text { Nomenclature } & \text { xvi }\end{array}$

Chapter 1 Introduction 1

Chapter 2 Literature Review 3

2.1 Modes of Transition . . . . . . . . . . . . . . . . . . . 3

2.1.1 Natural Transition . . . . . . . . . . . . . . 4

2.1.2 Bypass Transition . . . . . . . . . . . . . 5

2.1.3 Separation Bubble Transition ... . . . . . . . . . . . 7

2.2 The Theory of Turbulent Spots . . . . . . . . . . . . . . . 11

2.3 Review of Transition Modeling Techniques ... . . . . . . . . . 15

2.3.1 Direct Numerical Simulation . . . . . . . . . . . . . . 15

2.3.2 Low Reynolds number Turbulence Models . . . . . . . . . . . 15

2.3.3 Large Eddy Simulation . . . . . . . . . . . . . . . . . 17

2.4 Semi-Empirical Transition Models . . . . . . . . . . . . . . . . . 18

2.4 .1 Transition Inception . . . . . . . . . . . . . . . . 18

2.4 .2 Transition Length . . . . . . . . . . . . . . . . . . . 23 
2.5 Additional Conditions Influencing Transition . . . . . . . . . . . . . . 30

2.5.1 Surface curvature . . . . . . . . . . . . . . . . . . . 30

2.5.2 Periodic Unsteady and Wake-Induced Transition . . . . . . . . 32

2.5.3 Surface Roughness . . . . . . . . . . . . . . . . . 35

2.6 Numerical Simulation . . . . . . . . . . . . . . . . . . . . . . . . . . . 39

Chapter $3 \quad$ Experimental Setup and Data Analysis 42

3.1 Test Section Configuration . . . . . . . . . . . . . . . . . . . 42

3.1.1 Control of Flow Incidence . . . . . . . . . . . . . . . 43

3.1.2 Two-Dimensionality of the Flow . . . . . . . . . . . . . . . 44

3.1.3 Test Surfaces . . . . . . . . . . . . . . . . . . 44

3.1.4 Variation of Free-stream Turbulence . . . . . . . . . . . . . . . 47

3.1.5 Periodic-Unsteady Flow Generator . . . . . . . . . . . . . . 50

3.2 Instrumentation . . . . . . . . . . . . . . . . . . . 51

3.2 .1 Surface Instrumentation . . . . . . . . . . . . . . . 51

3.2.2 Hot-Wire Measurements . . . . . . . . . . . . . . . . 52

3.3 Data Analysis . . . . . . . . . . . . . . . . . . . . 56

3.3.1 Intermittency Distributions . . . . . . . . . . . . . 56

3.4 Summary of Experimental Uncertainties . . . . . . . . . . . . . 62

Chapter 4 Effects of Pressure Gradient and Free-Stream Turbulence on Attached Flow Transition $\quad 64$

4.1 Test Matrix . . . . . . . . . . . . . . . . . . . . . 65

4.2 Results and Discussion . . . . . . . . . . . . . . . . . 70

4.2 .1 Transition Inception _. . . . . . . . . . . . . . . 71

4.2.2 Streamwise Intermittency Distributions . . . . . . . . . . . . . 78

4.3 Summary . . . . . . . . . . . . . . . . . . . . . . 86

Chapter 5 Effects of Free-Stream Turbulence and Periodic Unsteadiness on Separation Bubble Transition 88 
5.1 Test Matrix . . . . . . . . . . . . . . . . . . . . . . . . . . . . . . 90

5.2 Results and Discussion . . . . . . . . . . . . . . . . . . . 93

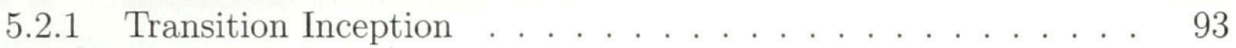

5.2.2 Streamwise Intermittency Distributions . . . . . . . . . . . . 94

5.2.3 Comparison to Existing Models . . . . . . . . . . . . . . 98

5.2.4 Transient Response of the Separation Bubble . . . . . . . . 100

5.3 Summary . . . . . . . . . . . . . . . . . . . . . . 107

Chapter 6 Effects of Surface Roughness and Free-Stream Turbulence on Boundary Layer Transition 109

6.1 Geometry of the Rough Surfaces . . . . . . . . . . . . . . . . . 110

6.2 Test Matrix . . . . . . . . . . . . . . . . . . . . . . . . . . 113

6.3 Results and Discussion . . . . . . . . . . . . . . . . . . . 116

6.3.1 Effects of Surface Roughness Height and Free-Stream Turbulence116

6.3 .2 Transition Inception . . . . . . . . . . . . . . . . . . . 122

6.3.3 Effects of Roughness Geometry _. . . . . . . . . . . . 125

6.3 .4 Spectral Analysis . . . . . . . . . . . . . . . . 130

6.3.5 Transition Length . . . . . . . . . . . . . . . . . 136

6.4 Summary . . . . . . . . . . . . . . . . . . . . . . . . 141

Chapter 7 Large-Eddy Simulation of Transition in a Separation Bubble 143

7.1 Numerical Method . . . . . . . . . . . . . . . . . . . . . . . . 144

7.1.1 Computational Domain and Boundary Conditions . . . . . . 144

7.1 .2 Solution Method . . . . . . . . . . . . . . . . . . 146

7.1 .3 Computational Grid . . . . . . . . . . . . . . 146

7.1 .4 Initial Conditions . . . . . . . . . . . . . . . . . . . 149

7.2 Results and Discussion . . . . . . . . . . . . . . . . . . . . 149

7.2 .1 Time-Averaged Flow Field . . . . . . . . . . . . . . . . 149

7.2 .2 Transient Flow Development . . . . . . . . . . . . . . . 152 
7.3 Summary . . . . . . . . . . . . . . . . . 158

$\begin{array}{lll}\text { Chapter } 8 & \text { Modeling Boundary Layer Transition } & 159\end{array}$

8.1 Description of Experiments . . . . . . . . . . . . . . . . . 160

8.2 Proposed Model . . . . . . . . . . . . . . . . . . . . . . 163

8.2.1 Location of Transition Inception . . . . . . . . . . . . . . 163

8.2 .2 Length of Transition . . . . . . . . . . . . . . . . 169

8.2.3 Effect of Surface Roughness on Transition Length . . . . . . . 177

8.3 Summary . . . . . . . . . . . . . . . . . . . . 179

$\begin{array}{ll}\text { Conclusions } & 181\end{array}$

$\begin{array}{ll}\text { References } & 184\end{array}$

$\begin{array}{lll}\text { Appendix A Test Section Ceiling Geometry } & 201\end{array}$

Appendix B Streamwise Variation of Free-Stream Turbulence 203

$\begin{array}{lll}\text { Appendix C Wavelet Analysis } & 208\end{array}$

Appendix D Digital Material rear cover

D.1 Data Reduction Programs

D.2 Large Eddy Simulation - Definition and Results Files 


\section{Figures}

Figure 2.1 Neutral stability curve for a Blasius Boundary Layer . . . . . 5

Figure 2.2 The Kelvin-Helmholtz Instability . . . . . . . . . . . . . . . . . 8

Figure 2.3 Schematic representation of long and short separation bubbles 10

Figure 2.4 Inception and growth of turbulent spots . . . . . . . . . . . 11

Figure 2.5 Destabilizing influence of concave curvature . . . . . . . . . . 31

Figure 2.6 Wake impingement in an axial turbine . . . . . . . . . . . . . . 33

Figure 2.7 Surface roughness at the leading edge of an HP turbine nozzle and near the suction peak of an LP turbine blade . . . . . . 36

Figure 3.1 Schematic of the wind tunnel test section . . . . . . . . . . . 43

Figure 3.2 Surface pressure measurements at three spanwise locations . . 45

Figure 3.3 Surface-normal variation of reference free-stream turbulence $\left(R e_{L}=470,000\right) \ldots \ldots \ldots \ldots$

Figure 3.4 Sample hot-wire calibration curve . . . . . . . . . . . . . . . . 53

Figure 3.5 Typical hot-wire signal in the boundary layer, and the deduced local flow state . . . . . . . . . . . . . . . . . . 59

Figure 3.6 Comparison of $\gamma(x)$ values obtained through $v-t$ traces and $H(x)$ distribution . . . . . . . . . . . . . . . 62

Figure 4.1 Surface static pressure distributions . . . . . . . . . . . . . 66

Figure 4.2 Streamwise variation of the acceleration parameter . . . . . . 69

Figure 4.3 Upstream movement of the transition inception location with increasing flow Reynolds number . . . . . . . . . . . 72 
Figure 4.4 Upstream movement of the transition inception location with increasing turbulence intensity . . . . . . . . . . . . . .

Figure 4.5 Comparison of experimental values of $R e_{\theta_{t s}}$ to the correlation of Abu-Ghannam \& Shaw (1980) . . . . . . . . . . . . 75

Figure 4.6 Comparison of $R e_{\theta_{t s}}$ to the correlations of Hourmouziadis (1989) and Mayle (1991) . . . . . . . . . . . . . .

Figure 4.7 Streamwise variation of the pressure gradient parameter $\lambda_{\theta}$ within the transition region . . . . . . . . . . . 80

Figure 4.8 Sensitivity of the intermittency distribution to $\lambda_{\theta} \ldots \ldots . . \quad 80$

Figure 4.9 Effect of turbulence intensity on the rate of transition . . . . . 81

Figure 4.10 Effect of flow Reynolds number on the rate of transition . . . 82

Figure 4.11 Comparison of measured intermittency distributions to the model of Solomon et al. (1996) . . . . . . . . . . . . . .

Figure 5.1 Streamwise variation in acceleration parameter . . . . . . . . . 93

Figure 5.2 Variation in the time-averaged streamwise intermittency distribution with Strouhal number . . . . . . . . . . . 96

Figure 5.3 Periodic turbulent spot development in the boundary layer . . 98

Figure 5.4 Observed experimental variations in $R e_{t s}$ and $R e_{r}$ with $R e_{s}$, with predictions of Hatman \& Wang and Yaras . . . . . . . . 99

Figure 5.5 Ensemble-averaged velocity profiles at the phases of maximum and minimum free-stream velocity $\left(R e_{L}=350,000\right) \ldots . . .101$

Figure 5.6 Ensemble-averaged velocity profiles at the phases of maximum and minimum free-stream velocity $\left(R e_{L}=470,000\right) \ldots . . .102$

Figure 5.7 Streamwise variation of steady and ensemble-averaged displacement thickness at the maximum and minimum free-stream velocity phases $-R e_{L}=350,000 \ldots \ldots$. . . . 104 
Figure 5.8 Streamwise variation of steady and ensemble-averaged displacement thickness corresponding to the maximum and minimum free-stream velocity phases $-R e_{L}=470,000 \ldots \ldots 105$

Figure 6.1 Streamwise distribution of the acceleration parameter . . . . 115

Figure 6.2 Variation of the time-averaged streamwise velocity field with pressure distribution . . . . . . . . . . . . . . 115

Figure 6.3 Distributions of boundary layer displacement thickness . . . . 119

Figure 6.4 Distributions of boundary layer shape factor . . . . . . . . . . 120

Figure 6.5 Sensitivity of the transition inception location in separation bubbles to surface roughness and free-stream turbulence . . . 124

Figure 6.6 Fourier power spectrum of $u^{\prime} / U_{\text {ref }}$ for $R e_{L}=350,000, C_{P 1}$ pressure distribution . . . . . . . . . . . . . . . . . . . 131

Figure 6.7 Comparison of observed dominant frequency to the correlation of Walker (1989) (Eq. 6.5) . . . . . . . . . . . . . . . . 133

Figure 6.8 Growth rate of disturbance energy in the $u^{\prime} / U_{\text {ref }}$ spectrum at $f_{M A} \ldots \ldots \ldots \ldots \ldots \ldots$

Figure 6.9 Comparison of hot-wire signals in the separation bubble for the $C_{P 1}$ and $C_{P 4}$ pressure distributions $\ldots \ldots \ldots \ldots$

Figure 6.10 Streamwise distributions of intermittency - separation bubble

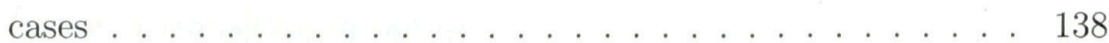

Figure 6.11 Streamwise distributions of intermittency - attached flow cases 139

Figure 6.12 Cross-stream distributions of intermittency for $k_{r m s}=53 \mu \mathrm{m}$ and $k_{r m s}=185 \mu \mathrm{m} \ldots \ldots \ldots \ldots \ldots$

Figure 7.1 Schematic of the computational domain . . . . . . . . . . . . . 145

Figure 7.2 LES and experimental time-averaged velocity field in the region of the separation bubble . . . . . . . . . . . . 150

Figure 7.3 LES and Experimental power spectra of $u^{\prime} / U_{\text {ref }}$ at various locations in the separation bubble ............ 15 
Figure 7.4 Growth rates of disturbances at the dominant frequency . . . 152

Figure 7.5 Streamwise velocity contours at mid-span during one full cycle of the dominant frequency . . . . . . . . . . . . . 153

Figure 7.6 Magnified view of streamwise velocity contours at mid-span and $t=t_{0}+3 T / 4 \ldots \ldots \ldots \ldots \ldots \ldots \ldots \ldots \ldots \ldots \ldots \ldots \ldots \ldots$

Figure 7.7 Instantaneous streamlines demonstrating shear layer wavering at the T-S wavelength $\left(\lambda_{f}\right) \ldots \ldots \ldots \ldots$

Figure 7.8 Contours of spanwise vorticity at mid-span at $t_{0}$ and $t_{0}+T / 2 \quad 155$

Figure 7.9 Magnified view of spanwise vorticity at mid-span and $t=t_{0} \quad . \quad 156$

Figure 7.10 Spanwise vorticity at $x-x_{s}=81 \mathrm{~mm}$ and $t=t_{0} \ldots \ldots$

Figure 7.11 Schematic representation of the vortex shedding process . . . . 157

Figure 8.1 Comparison of present experimental results to the experimental data and correlation of Abu-Ghannam and Shaw . . . . . . . 166

Figure 8.2 Sensitivity of the transition inception location in the separation bubble to free-stream turbulence (smooth surfaces) . . . . . . 168

Figure 8.3 Sensitivity of the transition inception location to surface roughness and free-stream turbulence . . . . . . . . . . . . 169

Figure 8.4 Variation of the spot inception parameter with $\lambda_{\theta_{t s}}$ and $T F \quad . \quad 171$

Figure 8.5 Variation of the spot production parameter with the shape factor at transition inception . . . . . . . . . . . . . 173

Figure 8.6 Variation of shape factor in the laminar boundary layer with free-stream turbulence . . . . . . . . . . . . . . . . . . . 174

Figure 8.7 Variation of $H_{t s}$ with turbulence level and $R e_{\theta_{s}}$ for separation bubble transition .................... . . 175

Figure 8.8 Intermittency distributions for two separated flow transition cases 176 Figure 8.9 Intermittency distributions for two attached flow transition cases 178

Figure A.1 Schematic of test section ceiling geometry . . . . . . . . . 202 
Figure B.1 Variation of free-stream turbulence intensity $-C_{P 1}$ pressure distribution; no grid installed . . . . . . . . . . . . . . 204

Figure B.2 Variation of free-stream turbulence intensity $-C_{P 1}$ pressure distribution; turbulence grid \#1 . . . . . . . . . . . . . . . . 204

Figure B.3 Variation of free-stream turbulence intensity $-C_{P \mathbf{1}}$ pressure distribution; turbulence grid \#2 . . . . . . . . . . . . . . . . 204

Figure B.4 Variation of free-stream turbulence intensity $-C_{P \mathbf{1}}$ pressure distribution; turbulence grid \#4 . . . . . . . . . . . . .

Figure B.5 Variation of free-stream turbulence intensity $-C_{P \mathbf{2}}$ pressure distribution; turbulence grid \#4 . . . . . . . . . . . . . . 205

Figure B.6 Variation of free-stream turbulence intensity $-C_{P 2}$ pressure distribution; turbulence grid \#5 . . . . . . . . . . . . . 205

Figure B.7 Variation of free-stream turbulence intensity $-C_{P 3}$ pressure distribution; no turbulence grid . . . . . . . . . . . . .

Figure B.8 Variation of free-stream turbulence intensity - $C_{P 3}$ pressure distribution; turbulence grid \#1 . . . . . . . . . . . . . . . 206

Figure B.9 Variation of free-stream turbulence intensity $-C_{P 3}$ pressure distribution; turbulence grid \#2 . . . . . . . . . . . . . . . 206

Figure B.10 Variation of free-stream turbulence intensity $-C_{P \mathbf{3}}$ pressure distribution; turbulence grid \#3 . . . . . . . . . . . . . . . .

Figure B.11 Variation of free-stream turbulence intensity $-C_{P 3}$ pressure distribution; turbulence grid \#4 . . . . . . . . . . . . . . 207

Figure B.12 Variation of free-stream turbulence intensity $-C_{P 4}$ pressure distribution; no grid installed . . . . . . . . . . . 207

Figure C.1 Schematic representation of the wavelet transform process . . 210

Figure C.2 Comparison of wavelet-averaged and Fourier power spectra . . 211 


\section{Tables}

Table 3.1 Surface scan areas and resolutions . . . . . . . . . . . . . . . . 47

Table 3.2 Turbulence generator specifications . . . . . . . . . . . . . . . . . 48

Table 3.3 Summary of experimental uncertainties . . . . . . . . . . 63

Table 4.1 Flow Reynolds number, type of pressure distribution, and freestream turbulence levels . . . . . . . . . . . . . . . . 67

Table 4.2 Streamwise position and flow parameters at transition inception 71

Table 5.1 Free-stream conditions . . . . . . . . . . . . . . . . . . 92

Table 5.2 Time-averaged streamwise positions and flow parameters at points of separation, reattachment, transition inception, and transition completion . . . . . . . . . . . . . . . 95

Table 6.1 Measured surface roughness parameters . . . . . . . . . . . . . 111

Table 6.2 Test matrix for rough surface measurements . . . . . . . . . . . . 114

Table 6.3 Rough surface separation bubble transition data $\left(C_{P 1}\right.$ pressure distribution) . . . . . . . . . . . . . . . . . 117

Table 6.4 Rough surface attached flow transition data $\left(C_{P 1}\right.$ pressure distribution) . . . . . . . . . . . . . . . . . . 118

Table 6.5 Variation in transition inception locations $(\mathrm{mm})$ with surface roughness and free-stream turbulence: $R e_{L}=350,000 \ldots 123$

Table 6.6 Variation in transition inception locations $(\mathrm{mm})$ with surface roughness and free-stream turbulence: $R e_{L}=470,000$. . . . 
Table 6.7 Low free-stream turbulence separation bubble flow and surface conditions $\left(C_{P 1}\right.$ pressure distribution, $\left.T u_{\text {ref }}=0.4-0.9 \%\right) \ldots .127$

Table 6.8 Low free-stream turbulence separation bubble flow and surface conditions $-C_{P 4}$ pressure distribution, $T u_{\text {ref }}=0.4-0.9 \% \ldots 128$

Table 7.1 Comparison of computational grids used in LES and DNS studies of transitional and turbulent flows . . . . . . . . . . . 147

Table 8.1 Summary of experimental configurations . . . . . . . . . . . . 162

Table 8.2 Flow parameters at transition inception - attached flow test cases 164

Table 8.3 Flow parameters at separation and transition inception $-C_{P 1}$ pressure distribution . . . . . . . . . . . . . . . . 165

Table 8.4 Flow parameters at separation and transition inception $-C_{P 4}$ pressure distribution . . . . . . . . . . . . . . 165

Table A.1 Test section ceiling geometry adjustment bar spacing . . . . . . 202

Table A.2 Test section ceiling geometry settings (angles in degrees) . . . . 202 


\title{
Nomenclature
}

\author{
$c$ \\ blade chord \\ $C_{P}$ \\ pressure coefficient $C_{P}=\frac{P-P_{r e f}}{\frac{1}{2} \rho U_{r e f}^{2}}$ \\ $C_{P 1} \ldots C_{P 4}$ \\ identifiers for the type of pressure distribution \\ $d$ \\ parameter quantifying the thickness of a hyperbolic tangent \\ shear layer $(\mathrm{m})$ \\ $F\left(\lambda_{\theta}\right)$ \\ pressure gradient function in the model of Abu-Ghannam \& \\ Shaw (Eq. 2.5) \\ $f$ \\ frequency $(\mathrm{Hz})$ \\ $g_{x}, g_{z}$ \\ growth functions of turbulent spots in Emmons model (Eq. 2.1) \\ $H$ \\ boundary layer shape factor $H=\delta^{*} / \theta$ \\ $k_{r m s}$ \\ RMS roughness height ( $\mu \mathrm{m}$, Eq. 6.1) \\ $L$ \\ length of test surfaces $(L=1220 \mathrm{~mm})$ \\ $N$ \\ number of surface measurement samples, (Eqs. 6.1 \& 6.3); \\ spot production rate parameter, (Eq. 2.22) \\ $n$ \\ number of times the roughness distribution crosses the mean \\ elevation, (Eq. 6.3); \\ turbulent spot production rate $(m s)^{-1}$, (Eqs. 2.3 and 2.17); \\ $P_{\text {ref }}$ \\ reference static pressure, measured $10 \mathrm{~mm}$ upstream of the test \\ surface leading edge \\ $P S D$ \\ power spectral density of $u^{\prime} / U_{\text {ref }}$ \\ $R e_{L}$ \\ reference Reynolds number based on $L$ and $U_{r e f}$ \\ $R e_{s}$ \\ Reynolds number based on $x_{s}$ and $U_{e_{s}}$
}




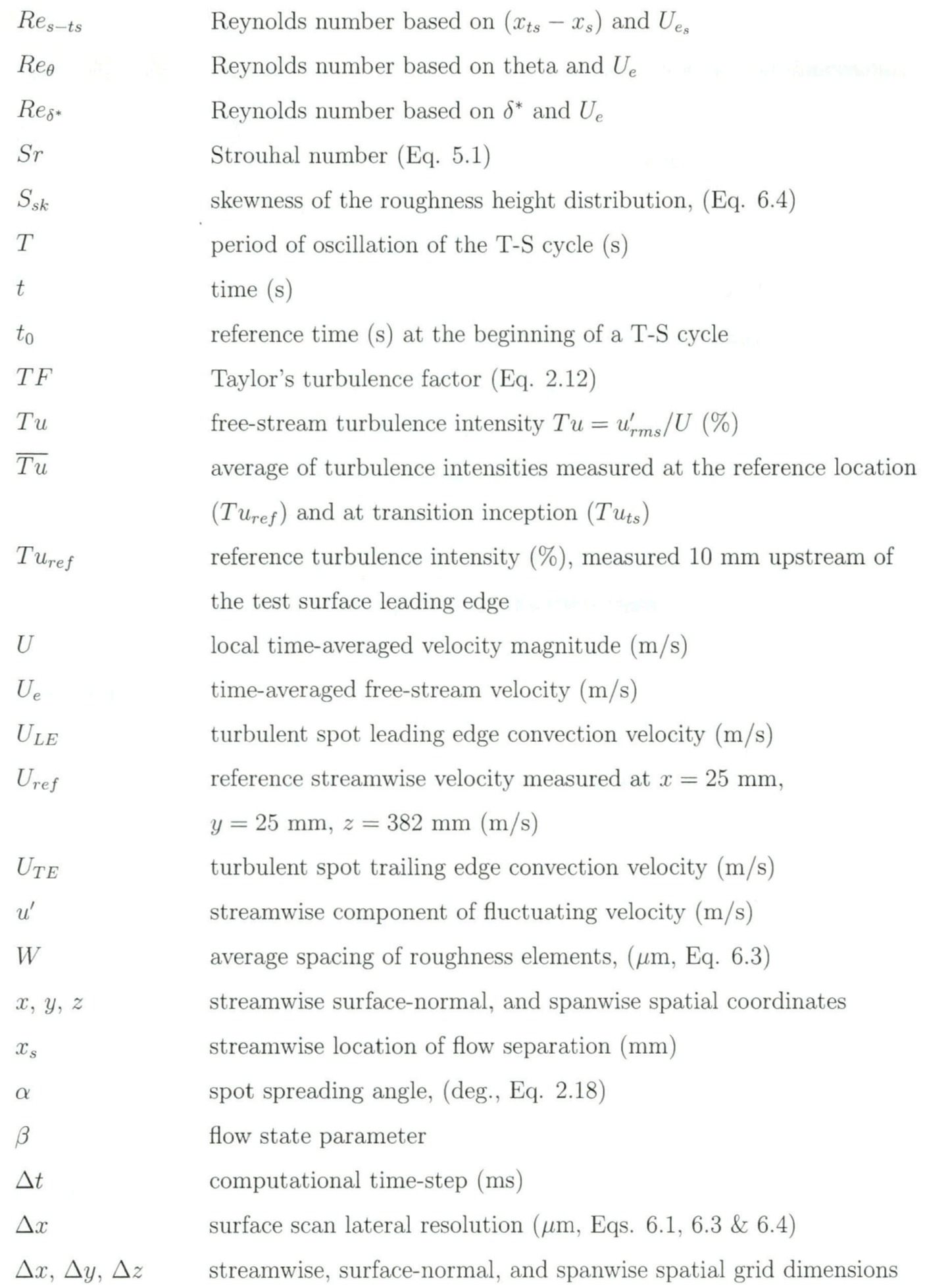


$(\mathrm{m})$

$\Delta x^{+}, \Delta y^{+}, \Delta z^{+}$streamwise, surface-normal, and spanwise spatial grid dimensions, expressed in wall units

$\delta^{*} \quad$ boundary layer displacement thickness $(\mathrm{mm})$

$\gamma \quad$ boundary layer intermittency

$\eta \quad$ acceleration parameter (Eq. 4.1)

$\kappa \quad$ wave number corresponding to $f_{M A}\left(\kappa=2 \pi / \lambda_{f} \mathrm{~m}^{-1}\right)$

$\Lambda_{s} \quad$ integral length scale of free-stream turbulence (mm)

$\lambda_{f} \quad$ fundamental instability wavelength $(\mathrm{m})$

$\begin{array}{ll}\lambda_{\theta} & \text { Thwaites' pressure-gradient parameter (Eq. 2.7) }\end{array}$

$\mu \quad$ mean elevation of rough surfaces (Eq. 6.2)

$\nu \quad$ kinematic viscosity $\left(\mathrm{m}^{2} / \mathrm{s}\right)$

$\sigma \quad$ spot propagation parameter, (Eq. 2.19)

$\theta \quad$ boundary layer momentum thickness ( $\mathrm{mm}$ )

$\omega_{z} \quad$ spanwise vorticity $\left(\mathrm{s}^{-1}\right)$

\section{Subscripts:}

$e$

$M A$

$r$

ref

$s$

ts

Acronyms:

$\mathrm{K}-\mathrm{H}$

RMS

T-S free-stream

maximum amplification rate of T-S waves

reattachment

reference condition

separation

start of transition

\section{Miscellaneous:}

Kelvin-Helmholtz

root-mean-square

Tollmien-Schlichting

over-bar denotes time averaging of variable 


\section{Chapter 1}

\section{Introduction}

Ever since the flow visualization experiments performed by Reynolds (1883), it has been well known that an initially laminar flow may spontaneously become turbulent. This transition process occurs more rapidly and readily as the flow Reynolds number is increased. At the high Reynolds numbers typical of aircraft airfoils, the transition region typically covers only a small portion of the airfoil surface, and the assumption of a point transition from a laminar to a turbulent state is adequate for predicting distributions of skin friction and pressure. However, at the relatively low Reynolds numbers encountered in gas turbine blade rows, the transition of the boundary layer from a laminar to a fully turbulent state can account for up to $80 \%$ of the blade surface (Turner, 1971). Therefore, for turbomachines, accurate modeling of the transition region is crucial to predicting aerodynamic losses and heat transfer rates at the blade surfaces. However, prediction of the transition process is greatly complicated by the wide range of flow and surface conditions with which these machines operate. The relative importance of these conditions has been 
the focus of numerous research efforts for several decades, and will likely remain so for several more decades to come.

For the purposes of engineering predictions, the parameters of primary interest when considering transitional boundary layers are the locations of transition inception and completion. Among the earliest known systematic investigations are the studies of Schubauer \& Skramstad (1948) and Hall \& Hislop (1938), who documented Reynolds numbers at the start and end of transition of the boundary layer developing over a flat surface. However, these early experiments were conducted under low freestream turbulence conditions, and in the absence of streamwise pressure gradients or additional disturbances. The transition process is known to depend on a number of flow and surface conditions, as will be discussed in Section 2.5. These conditions include the pressure distribution imposed on the boundary layer, surface curvature and roughness, and free-stream turbulence. In turbomachines, transition is also significantly affected by periodic impingement of wakes shed from upstream components, resulting in periodic variations in the free-stream velocity and turbulence levels. Although recent transition studies have examined the effects of an increasing number of these parameters, many aspects of boundary layer transition are still poorly understood. The present research program has been undertaken toward an improved understanding, and improved prediction, of the transition process. 


\section{Chapter 2}

\section{Literature Review}

\subsection{Modes of Transition}

Boundary layer transition has traditionally been classified as occurring in one of three modes: natural transition, bypass transition, and separation bubble transition. Each of these modes is likely to occur in turbomachines, and each is affected differently by the free-stream and surface disturbances imposed on the flow. Regardless of whether transition takes place in an attached boundary layer or in a separated shear layer, and in an environment with low or high levels of disturbances, the transition process is typically preceded by one or more types of instability. Additionally, in order for such an instability to grow, a disturbance must first be present. The initial response of the boundary layer to external disturbances is governed by the problem of receptivity. Since the nature of the instability has a profound effect on the ensuing transition process, the mechanisms encountered in the present research are described briefly in the following sections. As the focus of this research is on the transition process itself, detailed discussions of receptivity and 
stability are beyond the scope of the current work. The stability of fluid flows is discussed in a number of textbooks, such as those of Schlichting \& Gersten (2000), Chandrasekhar (1961), and Schmid \& Henningson (2001), and also in the report of Mack (1984). Discussions of receptivity can be found in the works of Reshotko (1976), Goldstein (1985), Ruban (1985), and Johnson (2003).

\subsubsection{Natural Transition}

Natural transition is the dominant mode of transition in attached flows with low or moderate free-stream turbulence. Near the leading edge of a streamlined body, the boundary layer is very thin, and any small disturbances that penetrate into the boundary layer are quickly damped by viscous forces. However, as the laminar boundary layer grows in thickness, it becomes more sensitive even to very small disturbances. If the thickness of the boundary layer, or more precisely the Reynolds number based on momentum thickness $\left(R e_{\theta}\right)$, reaches a certain critical value, disturbances within a certain range of frequencies can be amplified rather than damped, and form two-dimensional instability waves. These waves were first predicted theoretically by Tollmien and Schlichting (Tollmien, 1929; Schlichting \& Gersten, 2000), after whom these instabilities are now named, and were later produced in the experiments of Schubauer \& Skramstad (1948). For a zero-pressure-gradient boundary layer, the critical value of $R e_{\theta}$ at which these Tollmien-Schlichting (T-S) waves may begin to be amplified is $R e_{\theta}=163$, as shown by the locus of neutral stability in Fig. 2.1. After a relatively long stage of linear spatial growth, the T-S waves are subject to a secondary instability, characterized by a three-dimensional 


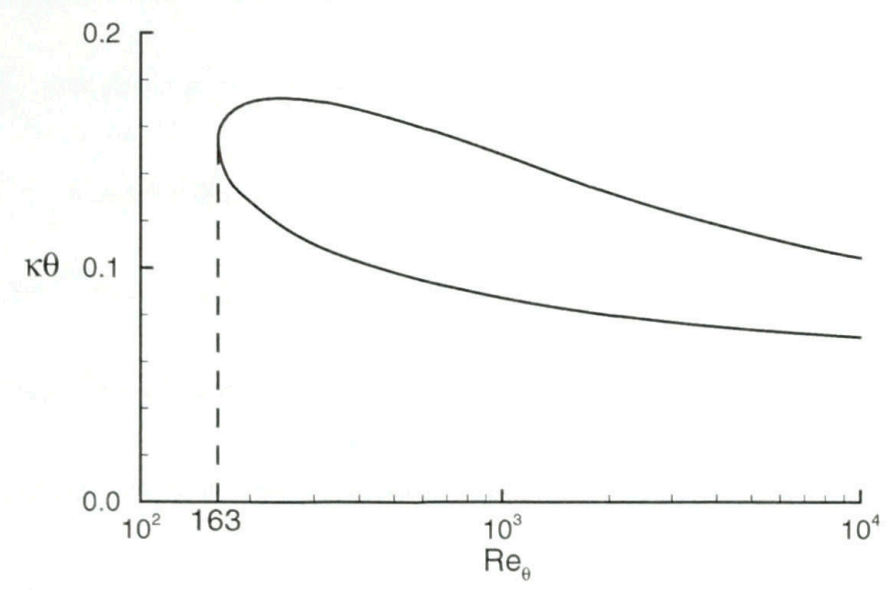

Figure 2.1: Neutral stability curve for a Blasius boundary layer (from Harrar \& Osborne, 2003)

disturbance field and exponential amplification of disturbances. The shape of the vortical structures produced during this phase initially resembles the Greek letter $\Lambda$. These $\Lambda$ vortices stretch and evolve into more longitudinal "hairpin" vortex structures, from which the first truly turbulent flow structures are formed. After their inception, these turbulent structures grow as they are convected downstream, until they merge and form a fully turbulent boundary layer, as will be described in Section 2.2.

\subsubsection{Bypass Transition}

Bypass transition is a process in which the stages of linear growth of instabilities are bypassed, due to the influence of large amplitude disturbances (Morkovin, 1969). This type of transition occurs commonly in turbomachines, due to the relatively high free-stream turbulence levels typically present in these flows. Bypass transition may also occur in flows over rough surfaces. Although the mechanism by which bypass transition is initiated is not yet fully understood, it is known that some of the assumptions in the stability analysis of natural transition 
are not valid when large disturbances are present. For example, disturbances at frequencies that are damped for the infinitesimally small initial amplitudes assumed in linear stability of natural transition (i.e. above or below the neutral stability curve of Fig. 2.1) have been observed to undergo a period of linear growth, followed by exponential decay (Reshotko, 2001; Butler \& Farrell, 1992; Schmid \& Henningson, 2001). Alternatively, certain wavelengths of disturbances may grow temporarily prior to the boundary layer reaching the critical momentum thickness Reynolds number for which Tollmien-Schlichting waves may be amplified (i.e. to the left of the neutral stability curve of Fig. 2.1). This so-called transient growth mechanism (Reshotko, 2001; Butler \& Farrell, 1992) has recently emerged as a mechanism that may possibly help to explain the phenomenon of bypass transition.

Regardless of how this process is initiated, the large amplitude disturbances introduced into the boundary layer, by turbulence or surface roughness, cause the stage of linear growth of natural instabilities, as described in the previous section, to be bypassed. In these circumstances, regions of three-dimensional turbulence appear in the boundary layer without any significant prior growth of two-dimensional Tollmien-Schlichting instability waves. Because of the environment of large disturbances, bypass transition inception typically occurs further upstream than in natural transition. However, once formed, the process by which the threedimensional disturbances grow into a fully turbulent boundary layer is similar to that for natural transition. 


\subsubsection{Separation Bubble Transition}

The aerodynamic loading of low-pressure turbine blades has increased dramatically in recent years, as designers strive to reduce the blade count, and thus the weight of these components, while maintaining a high work output. These efforts have led to more frequent occurrences of laminar separation on the suction side of these blades (Hodson, 1991), particularly in aircraft engines operating at high altitudes, where the blade chord Reynolds number may be as low as 20,000. The flow in a separated shear layer is inherently unstable, and often undergoes transition shortly after separation. After transition, the enhanced momentum exchange in the turbulent shear layer often causes reattachment of the flow. Since turbulent boundary layers are more tolerant of adverse pressure gradients, the turbulent boundary layer typically remains attached to the trailing edge of the blade.

\subsubsection{Stability Considerations for Separation Bubbles}

The majority of velocity distributions having an inflection point are unstable with respect to the inviscid Kelvin-Helmholtz (K-H) mechanism (Chandrasekhar, 1961). Thus, the K-H instability is the dominant transition mechanism in free shear layers. As with the T-S instability, the locus of neutral stability for the K-H mechanism can be predicted through linear stability analysis (e.g. Chandrasekhar, 1961). The K-H instability is characterized by a roll-up of the vortex sheet at the inflection point of the velocity profile, resulting in regions of concentrated vorticity, connected by a thin "braid" of weaker vorticity, as shown schematically in Fig. 2.2-b. The spacing between concentrated vortices is the fundamental wavelength of the K-H 


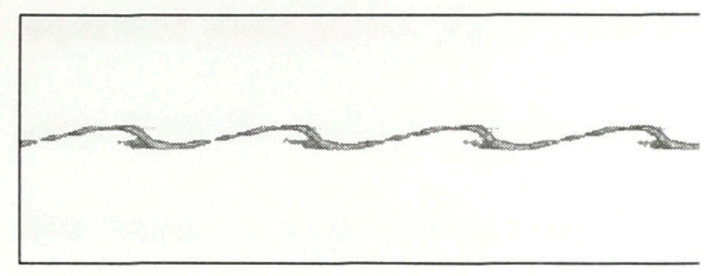

(a) Shear layer wavering

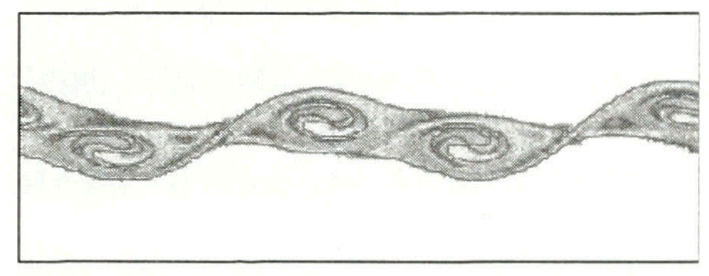

(c) Pairing

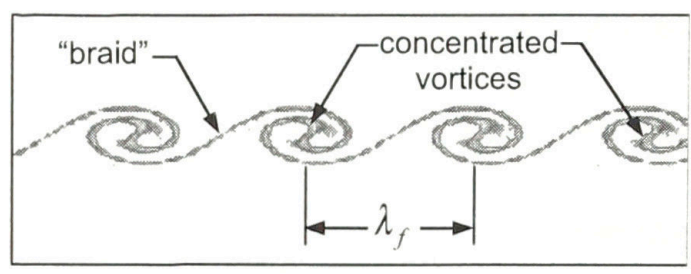

(b) Roll-up

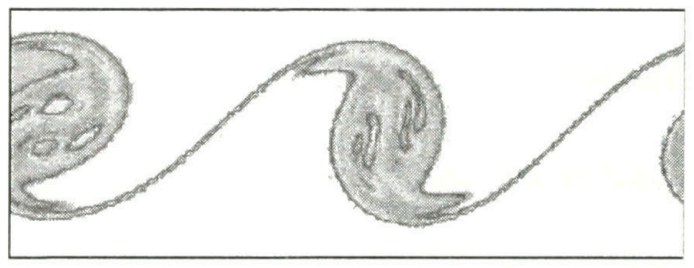

(d) Secondary roll-up

Figure 2.2: The Kelvin-Helmholtz Instability (adapted from Fringer 2004)

instability ( $\lambda_{f}$ in Fig. 2.2-b). As they grow in size, adjacent vortices may themselves undergo one or more similar roll-up, or pairing, processes, resulting in larger regions of vorticity, spaced at sub-harmonics of the fundamental wavelength (Fig. 2.2-d). Eventually, the vortex structure itself grows sufficiently to become unstable, at which point it breaks down into three-dimensional turbulent eddies.

Transition in a separation bubble occurs in the separated shear layer, and it has long been believed that separation bubble transition occurs exclusively via the KelvinHelmholtz instability, as in free shear layers. However, in small separation bubbles, the shear layer is located relatively close to the surface, and a significant effect of wall damping on the shear layer remains. In these instances, transition inception may be preceded by the growth of Tollmien-Schlichting (T-S) instability waves (e.g. present work, Volino \& Bohl, 2004; Volino, 2002-c), which break down and form turbulent spots characteristic of natural transition in attached boundary layers. The $\Lambda$ vortices normally associated with natural attached flow transition have also been observed in 
separated shear layers (Bao \& Dallmann, 2004). As the separated shear layer moves away from the surface, the damping of the wall becomes less pronounced, and the flow begins to more closely resemble a free shear layer, in which the inviscid KelvinHelmholtz (K-H) instability mechanism is expected to dominate (e.g Malkiel \& Mayle, 1996; Watmuff, 1999; Yang \& Voke, 2001; Spalart \& Strelets, 2000). Computational studies of separated boundary layers have shown that the distance of the shear layer from the surface, the thickness of the shear layer, and the flow Reynolds number are all factors affecting the dominant instability mode (e.g. Rist \& Maucher, 2002; Chandrasekhar, 1961). Laminar separation bubbles therefore share similarities with both attached boundary layers and free shear layers, and transition may be initiated by either the Kelvin-Helmholtz or Tollmien-Schlichting mechanisms. The relative roles of these instability mechanisms in the transition process are discussed further in Chapters 6 and 7 .

\subsubsection{Classification of Separation Bubbles}

Laminar separation bubbles have historically been classified as being either "long" or "short" (Gaster, 1967). Long separation bubbles are characterized by the existence of multiple recirculation zones (Fig. 2.3-a) and affect the overall pressure distribution over the surface. In contrast, short separation bubbles have only a local effect on the pressure distribution, and consist of a single laminar recirculating region within the bubble (Fig. 2.3-b), although a much smaller, turbulent recirculation zone (not shown in figure) was also observed by Hatman \& Wang (1999). Short separation bubbles typically occur at higher Reynolds numbers, or when milder adverse pressure 


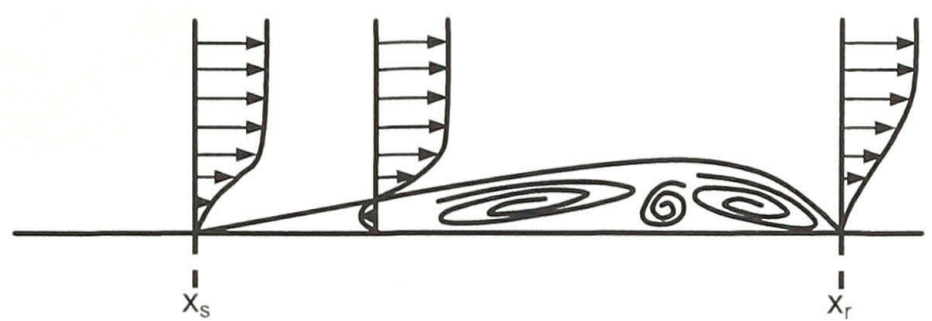

(a) Long bubble

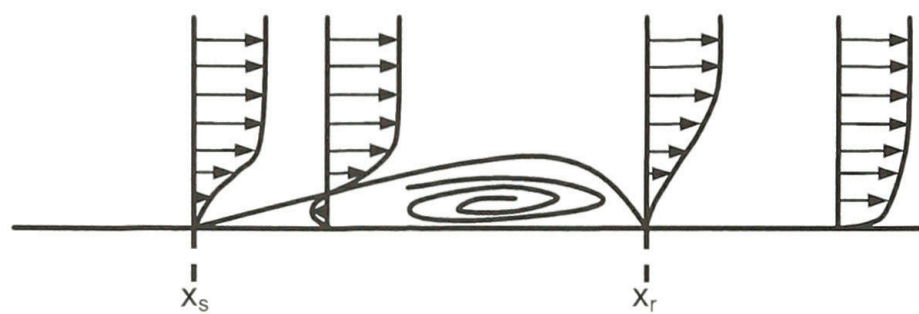

(b) Short bubble

Figure 2.3: Schematic representation of long and short separation bubbles (after Hatman \& Wang, 1999)

gradients are imposed upon the boundary layer. These conditions promote earlier reattachment of the transitional or turbulent shear layer. As the Reynolds number is decreased, or the pressure gradient is increased, a short separation bubble can suddenly "burst", and become a long bubble (Gaster, 1967), or fail to reattach altogether.

In addition to laminar separation bubbles, at relatively high flow Reynolds numbers and mild adverse pressure gradients, transition inception may precede the separation point. Other than the fact that transition inception precedes the separation point, this type of separation bubble exhibits a very similar behaviour to that of a "short" separation bubble (Hatman \& Wang, 1999). 


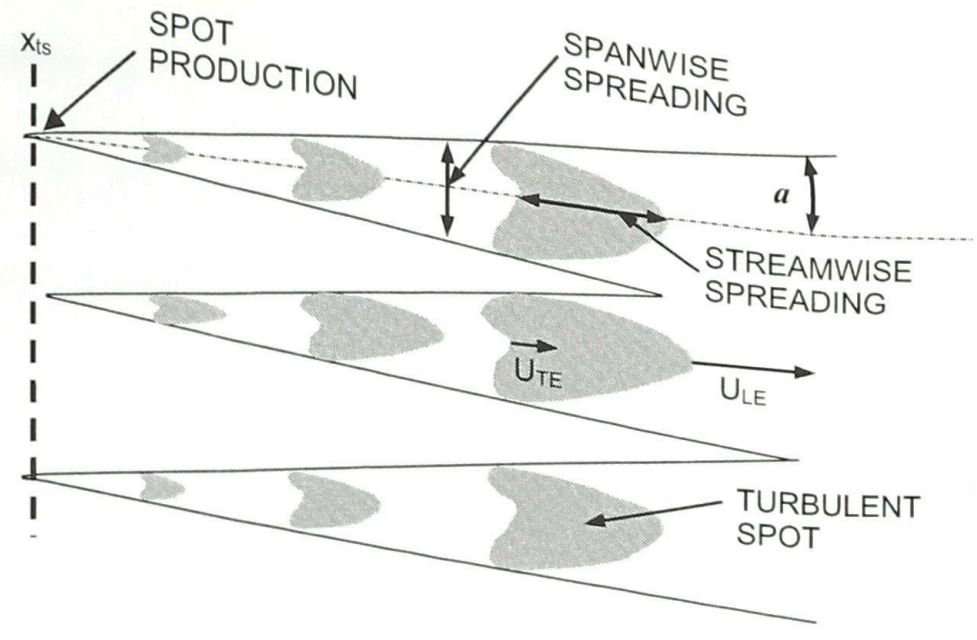

Figure 2.4: Inception and growth of turbulent spots

\subsection{The Theory of Turbulent Spots}

Fluid flows typically do not immediately become fully turbulent at the point where instability occurs, and as noted earlier, the transition region can account for a significant portion of the boundary layer flow field. Since the characteristics of laminar, transitional, and turbulent boundary layers are significantly different, it is important for designers to be able to locate the beginning and end of the transition zone. Emmons (1951) was the first to recognize that transition in attached boundary layers occurs through the production of turbulent "spots" that are roughly triangular when viewed in planform, with the tip pointing in the downstream direction (e.g. Emmons, 1951; Gostelow et al., 1996; Chong \& Zhong, 2003). Once formed, these spots spread in the streamwise, spanwise, and cross-stream directions as they are convected downstream, until they merge with each other to form a fully turbulent boundary layer (Fig. 2.4).

Emmons concluded that the flow at any point in the transition region at times 
appears laminar, and at other times turbulent, depending on whether the point lies within a turbulent spot at the instant considered. This was a significant discovery, since the accepted theory at the time was that the transition region consisted of a monotonic variation from a laminar to a turbulent state. Emmons showed that the length of the transition zone is dictated by the inception, convection and spreading rates of these turbulent spots. The parameter of primary interest in transition studies is the intermittency $(\gamma)$ of the boundary layer, defined as the probability that at any given time a point in the flow field is contained within a turbulent spot. In the laminar region, $\gamma=0$, and in a fully turbulent boundary layer, $\gamma=1$, except near the free-stream edge of the boundary layer, and in the viscous sub-layer near the solid boundary. Thus, in the transition region, the intermittency varies between 0 and 1 .

Ignoring the variations in the cross-stream direction, the intermittency of a two-dimensional (spanwise uniform) boundary layer may be mathematically expressed as (Emmons, 1951):

$$
\gamma(x)=1-e^{-\int_{0}^{x} n(x) d x \int_{0}^{x} g_{x}(x) d x \int_{0}^{x} g_{z}(x) d x}
$$

where $n$ represents the average inception rate of turbulent spots per unit span at the streamwise location $x$, and $g_{x}(x)$ and $g_{z}(x)$ respectively represent the local streamwise and spanwise spreading rates of these spots. Emmons assumed the spot production and spreading rates to be constant over the entire surface, leading to the following expression for the intermittency: 


$$
\gamma(x)=1-e^{-n g_{x} g_{z} x^{3}}
$$

Emmons' assumption of uniform spot production is not supported by the observation of Schubauer \& Klebanoff (1955), where a laminar boundary layer development was followed by sudden appearance of turbulent spots. These experiments led Narasimha (1957) to propose a "universal" intermittency model based on a hypothesis of concentrated breakdown, whereby spot inception occurs at random times and spanwise locations at a single streamwise position:

$$
\gamma(x)=1-e^{-\frac{n \sigma}{U_{e}}\left(x-x_{t s}\right)^{2}} \quad\left\{x>x_{t s}\right\}
$$

In this expression, $\sigma$ is a spot propagation parameter, given by:

$$
\sigma=U_{e}\left(\frac{1}{U_{T E}}-\frac{1}{U_{L E}}\right) \tan (\alpha)
$$

In Eq. 2.4, $\alpha$ is the lateral spot spreading half-angle, and $U_{L E}$ and $U_{T E}$ are the spot leading and trailing edge convection velocities, respectively (Fig. 2.4). Narasimha assumed these quantities to have constant values within the transition region to derive Eq. 2.3.

Recently, Johnson \& Fashifar (1994) observed that spot inception takes place within a finite band rather than at a single streamwise location. Another underlying assumption in the theories of Emmons and Narasimha is that the inception of a turbulent spot is not affected by the proximity of other spots. However, it 
has been demonstrated experimentally that turbulent spots are less likely to be produced in close proximity to each other (Johnson \& Fashifar, 1994). This is likely due to the existence of a calmed region following the passage of a turbulent spot (Johnson \& Fashifar, 1994; Chong \& Zhong, 2003). Despite evidence of deviations from Narasimha's assumptions, as will be demonstrated in Chapters 4 and 8, the level of success that has been achieved with transition models based on the concentrated breakdown hypothesis suggests these deviations to be of little importance in engineering predictions.

Since the publications of Emmons (1951) and Narasimha (1957), it has become clear that there are four parameters required to model the transition region:

1. The location of transition inception: $x_{t s}$

2. The rate of turbulent spot production: $n$

3. The spanwise spreading rate of turbulent spots: $\alpha$

4. The streamwise spreading rate of turbulent spots: $U_{e}\left(\frac{1}{U_{T E}}-\frac{1}{U_{L E}}\right)=\frac{\sigma}{\tan (\alpha)}$

The location of transition inception and the spot production rate are fixed for a given transition process. However, the spreading rates of turbulent spots may vary significantly within the transition region. Several methods for modeling these parameters are available, ranging from purely empirical models to highly refined numerical simulations. 


\subsection{Review of Transition Modeling Techniques}

\subsubsection{Direct Numerical Simulation}

The ultimate mathematical model for predicting the transition process already exists in the form of the unsteady Navier-Stokes equations. Until relatively recently however, lack of computing power has prevented the numerical solution of these equations with sufficient resolution to predict the details of turbulence production, convection, diffusion and dissipation processes in transitional or turbulent flows. Direct numerical simulations (DNS) are now being used as numerical wind tunnels to shed light onto fundamental features of transition and turbulence (Lang et al., 2004; Alam \& Sandham, 2000; Rai \& Moin, 1993; Maucher et al., 1999), which are extremely difficult to observe through physical experiments. However, the available computing power has imposed limits on the range of flow Reynolds numbers that can be considered in such simulations (Maucher et al., 1999; Alam \& Sandham, 2000), which will remain so for the foreseeable future.

\subsubsection{Low Reynolds number Turbulence Models}

There has been considerable research focusing on predicting transition through the use of turbulence models designed for low Reynolds numbers (e.g. Wilcox, 1977; Rodi \& Sheuerer, 1985; Schmidt \& Patankar, 1991). However, there appear to be no fundamental grounds for these models to properly capture the physics of the transition process, since they have been tailored to predict the near-wall region of fully turbulent boundary layers. Amplification of instabilities, and intermittent flow phenomena 
unique to the transition process, cannot as easily be modeled in a time-averaged sense as equilibrium boundary layer turbulence. The low-Re turbulence modeling approach also requires free-stream disturbances to be present, which limits this method to predicting bypass transition processes, triggered by diffusion of free-stream turbulence into the boundary layer. Savill (1992) showed that low-Re turbulence models fail to predict both the transition onset location and the length of the transition zone reliably. The latter trend was noted by Schmidt \& Patankar (1991), who also showed these models to be sensitive to the location of the inlet boundary to the computational domain and initialization of turbulence quantities. The poor performance of low Reynolds number turbulence models in attached flow is likely to prevail in separated flow transition as well.

There have been attempts to improve the transition prediction performance of low Reynolds number turbulence models through the use of intermittency functions (e.g. Savill, 1992). Such methods are more general than the basic low Reynolds number turbulence modeling approach, in the sense that they explicitly account for spot formation and growth rates. However, they retain the underlying assumption of similarity between the turbulence structure in the turbulent spots and in the fully turbulent boundary layer, and the location of transition inception needs to be estimated through other means, typically from empirical correlations. Hobson \& Weber (2000) attempted to use the turbulence model of Spalart \& Allmaras (1992), together with the intermittency function of Solomon et al. (1996) for separation bubble transition, and found that the internal structure of the bubble was not predicted well. More recently, transport equations have been developed for predicting 
the streamwise variation of intermittency rather than relying on empirical relations (e.g., Steelant \& Dick, 1996; Suzen \& Huang, 2000; Menter et al., 2004). The models of Suzen \& Huang (2000) and Menter et al. (2004) also account for the cross-stream variation of flow intermittency. The authors reported reasonable accuracy for the prediction of the transition length, with the transition inception location determined through an empirical correlation. However, in the context of the previous discussion on the use of low-Re turbulence models to predict the turbulence generation within turbulent spots, the author remains doubtful whether consistent prediction accuracy can be obtained with such models.

\subsubsection{Large Eddy Simulation}

Large eddy simulation (LES) has relatively recently emerged as a compromise between the excessive computing requirements of DNS, and the lack of general applicability of Reynolds-averaged Navier-Stokes (RANS) formulations to turbulent flows. In this method, the larger turbulent structures are computed explicitly, while the smaller scale turbulence is modeled, which is commonly referred to as sub-grid scale modeling (e.g. Lesieur \& Métais, 1996). This approach has been shown to have the potential to provide substantially more consistent prediction of turbulent flows than has been accomplished by modeling the complete range of turbulence scales (Rodi et al., 1997). Since a large range of turbulence scales naturally develop during such simulations, prediction of transition inception and turbulent spot propagation is theoretically within the capability of this method. This has been confirmed through case studies in the published literature (e.g. Rodi et al., 1997; Huai et al., 1997). 
However, the LES computing requirements for high-Re flows are within a range that makes LES unattractive for regular use in industry. Additionally, the feasibility of sub-grid scale turbulence models that are equally effective away as well as in close proximity of solid boundaries remains the focus of extensive research. Hence, it will be some time before this technique matches the reliability and efficiency of the well proven semi-empirical modeling approach for the prediction of the transition process.

Based on these observations, for the foreseeable future, semi-empirical tools appear to be the most accurate and time efficient means for modeling turbulent spot inception and propagation. The following sections review existing such models for predicting the inception location and length of transition. These studies provide the starting point for a new model that is proposed in Chapter 8, that accounts for the effects of a broader range of flow and geometric parameters, calibrated against an extensive set of experimental data.

\subsection{Semi-Empirical Transition Models}

\subsubsection{Transition Inception}

The first step in predicting a transition process is determining where transition inception will occur. For transition processes triggered by viscous (T-S) instability, this is equivalent to predicting the location at which turbulent spots are first produced. For transition processes initiated by the inviscid $(\mathrm{K}-\mathrm{H})$ instability, transition inception is the location where breakdown of the two-dimensional vortex structure described in Section 2.1.3 occurs. 
One of the shortcomings of linear stability analysis (Section 2.1) is that it predicts only whether a disturbance, if present, may be amplified. Thus, linear stability, on its own, is not a means by which transition inception may be predicted. Whether or not transition inception occurs depends on the disturbance environment of the flow. To predict this, we typically rely on empirical correlations derived from experiments.

\subsubsection{Attached Flows}

Perhaps the first parameter to be considered in studies related to boundary layer transition was the effect of the free-stream turbulence level. Early investigations, such as those of Hall \& Hislop (1938) and Schubauer \& Skramstad (1948), documented a significant effect of free-stream turbulence intensity on the development of the boundary layer over a flat plate. Although the turbulence levels considered in these studies were fairly low by turbomachinery standards $(T u<3 \%)$, increases in freestream turbulence levels were seen to cause a notable upstream movement of the transition inception point, as well as the location of transition completion. Later studies (e.g. Abu-Ghannam \& Shaw, 1980, and Martin et al., 1978) extended the range of turbulence levels to include those more representative of turbomachinery, and also included the effects of streamwise variations in pressure gradient on the development of the boundary layer. In these studies, adverse pressure gradients were seen to promote earlier transition inception, while favourable pressure gradients were noted to delay transition. This is largely attributable to the reduced rate of growth of the laminar boundary layer thickness in accelerated flows, as compared to decelerating 
flows.

In attached boundary layers, the widely accepted parameter for predicting the location of transition inception is the Reynolds number based on momentum thickness $\left(R e_{\theta}\right)$. Among the numerous transition inception formulations, the following one of Abu-Ghannam \& Shaw (1980) is cited most frequently in turbomachinery blade studies:

$$
R e_{\theta_{t s}}=163+e^{F\left(\lambda_{\theta}\right)\left(1-\frac{\overline{T u} \%}{6.91}\right)}
$$

where $\overline{T u}$ is the average of the turbulence intensities at the leading edge and the location of transition inception $\left(\overline{T u}=\frac{1}{2}\left(T u_{t s}+T u_{r e f}\right)\right)$, and $F\left(\lambda_{\theta}\right)$ is given by the following:

$$
\begin{array}{cc}
F\left(\lambda_{\theta}\right)=6.91+12.75 \lambda_{\theta}+63.64 \lambda_{\theta}{ }^{2} & \left\{\lambda_{\theta} \leq 0\right\} \\
F\left(\lambda_{\theta}\right)=6.91+2.48 \lambda_{\theta}+12.27 \lambda_{\theta}{ }^{2} & \left\{\lambda_{\theta}>0\right\}
\end{array}
$$

The pressure gradient parameter $\lambda_{\theta}$ is defined as (Thwaites, 1960):

$$
\lambda_{\theta}=\frac{\theta^{2}}{\nu} \frac{d U_{e}}{d x}
$$

Mayle (1991) and Hourmouziadis (1989) noted that for free-stream turbulence intensities above about $3 \%$, there is little effect of the pressure distribution on the location of transition inception, and that at turbulence levels typical 
of turbomachinery, the turbulence intensity is the dominant parameter affecting transition inception. These authors developed the following alternative formulations for use in moderate and high free-stream turbulence environments:

Hourmouziadis (1989):

$$
R e_{\theta_{t s}}=460 \overline{T u}^{-0.65}
$$

Mayle (1991):

$$
R e_{\theta_{t s}}=400 \overline{T u}^{-0.625}
$$

As noted by Mayle (1991), the correlation of Abu-Ghannam \& Shaw (1980) is artificially forced to correspond to the stability limit for natural transition $\operatorname{Re}_{\theta_{s}}=163$ for flows with high turbulence intensity, and therefore does not allow for prediction of bypass transition. Although the models of Mayle (1991) and Hourmouziadis (1989) do not account for the effects of pressure gradient, they are not limited by the stability criterion of $R e_{\theta_{s}}=163$.

\subsubsection{Separation Bubbles}

For transition in short separation bubbles, the location of transition inception has been found to correlate well with the state of the boundary layer at the point of separation. Several examples are given below:

Mayle (1991): 


$$
R e_{s-t s}=300 R e_{\theta_{s}}^{0.7}
$$

where $R e_{s-t s}$ is the Reynolds number based on the streamwise distance between the separation and transition inception locations $\left(R e_{s-t s}=U_{e_{s}}\left(x_{t s}-x_{s}\right) / \nu\right)$.

Roberts (1975):

$$
R e_{s-t s}=2.5 \times 10^{4} \log _{10}\left(\operatorname{coth} \frac{T F(\%)}{10}\right)
$$

In this equation, $T F$ is Taylor's (1939) turbulence factor, defined as:

$$
T F=T u_{r e f}\left(L / \Lambda_{s}\right)^{0.2}
$$

where $\Lambda_{s}$ is the integral length scale of free-stream turbulence, and $L$ is the length of the surface along which the boundary layer develops.

Davis et al. (1987)

$$
R e_{s-t s}=2.5 \times 10^{4} \log _{10}\left(\operatorname{coth}\left(0.1732 T u_{t s}(\%)\right)\right)
$$

Hatman \& Wang (1999):

$$
R e_{t s}=1.0816 R e_{s}+26,805
$$

Yaras (2002): 


$$
R e_{t s}=1.04 R e_{s}+6.3 \times 10^{4}\left(1-\tanh ^{3}\left(T F^{\prime}(\%)\right)\right)
$$

where $T F^{\prime}=\max (T F(\%), 1 \%)$

Among these models, Eqs. 2.14 and 2.15 have been found to be in better agreement with the experimental data measured during the course of the current study. However, this is not surprising, since the Reynolds number based on streamwise distance from the leading edge used in these models $\left(R e_{t s}\right)$ is not as sensitive to changes in the transition inception location as is $R e_{s-t s}$. These models are therefore not able to resolve the transition inception location with as high a degree of precision as those of Mayle (1991) and Roberts (1975). A substantial improvement to these models is presented in Chapter 8 .

\subsubsection{Transition Length}

\subsubsection{Attached Flows}

The length of the transition zone in attached flows (and in separated flows dominated by viscous instability) is dictated by the rates of production and spreading of turbulent spots. Since the work of Emmons (1951), numerous studies have documented the production and development of turbulent spots as they are convected downstream.

Many studies have documented the structure and spreading rates of spots in the horizontal plane (e.g. Gostelow et al., 1996; Zhong et al., 2002), or in the vertical plane of symmetry (Gostelow et al., 1996). More recently, measurements have 
been undertaken to fully document the three-dimensional spot structure (Chong \& Zhong, 2003). Such three-dimensional measurements have identified distinct regions of turbulence generation at the leading edge and along the lateral extremes of the spots. Through these studies, the local pressure gradient has been confirmed as the parameter dominating the spreading rate of turbulent spots.

Experimental studies have shown that the rate of turbulent spot production is affected by the local free-stream turbulence and streamwise pressure gradient (Fraser et al., 1994; Gostelow et al., 1994), and by surface roughness, as indicated indirectly by the measurements of Pinson \& Wang (1997). The random occurrence of turbulent spots prevents direct measurement of their inception rates. Thus, conclusions regarding the trends in spot inception rates must be deduced from the transition length and known or assumed spot propagation characteristics in the transition zone. The level of success in experimentally quantifying the inception rate of turbulent spots is therefore closely coupled to the extent of our understanding of their convection and spreading characteristics.

The intermittency model of Narasimha (Eq. 2.3) has been found to agree well with measurements in flows with both favourable and mild adverse pressure gradients (e.g. Narasimha, 1985; Gostelow et al., 1994; Devasia, 1974), provided that the pressure gradient does not vary significantly within the transition region. However, in turbomachines, transition inception may occur in a region of strong adverse or favourable pressure gradient, and the pressure gradient may also vary significantly within the transition region. Strong variations in pressure gradient within 
the transition region have been found to result in intermittency distributions which differ significantly from those described by Eq. 2.3. A model to predict this departure from the universal intermittency distribution, through local adjustment of the spot convection velocity, was first proposed by Chen \& Thyson (1971).

$$
\gamma(x)=1-e^{n \sigma\left(x-x_{t s}\right) \int_{x_{t s}}^{x} \frac{d x}{U(x)}}
$$

However, Walker et al. (1988) noted that this adjustment is insufficient to establish good agreement with experimental results. The failure of the model of Chen \& Thyson (1971) is attributed to the fact that the streamwise and spanwise spreading rates of turbulent spots vary significantly with $\lambda_{\theta}$, as was documented by Gostelow et al. (1996). Based on these findings, Solomon, Walker, \& Gostelow (1996) proposed the following expression:

$$
\gamma(x)=1-e^{-n \int_{x_{t s}}^{x} \frac{\sigma}{\tan (\alpha) U} d x \int_{x_{t s}}^{x} \tan (\alpha) d x}
$$

In this equation, $\frac{\sigma}{\tan (\alpha) U}$ and $\tan (\alpha)$ represent the streamwise and spanwise spreading of turbulent spots, respectively. The following expressions for the spot spreading characteristics were proposed by Gostelow et al. (1996), for $\lambda_{\theta}$ values between -0.06 and +0.08 , based on experimental data gathered from several studies (van Hest et al., 1994; Gostelow et al., 1993; Sankaran et al., 1986; Wyganski, 1981; Wyganski et al., 1982; Schubauer \& Klebanoff, 1955; Katz et al., 1990): 


$$
\begin{aligned}
\alpha & =4+\frac{22.14}{0.79+2.72 e^{47.63 \lambda_{\theta}}} \\
\sigma & =0.03+\frac{0.37}{0.48+3 e^{52.9 \lambda_{\theta}}}
\end{aligned}
$$

More recently, Eq. 2.19 was updated by (D'Ovidio et al., 2001) to extend the range of $\lambda_{\theta}$ to -0.12 :

$$
\sigma=0.024+\frac{0.604}{1+5 e^{66 \lambda_{\theta}}}
$$

The correlation proposed by Solomon et al. (1996) for the spot production rate is based on the measurements of Gostelow et al. (1994) for $\lambda_{\theta}<0$, and of Fraser et al. (1994) for $\lambda_{\theta}>0$ :

$$
\begin{array}{ll}
N=8.6 \times 10^{-4} e^{2.134 \lambda_{\theta_{t s}} \ln \left(T u_{t s}\right)-59.23 \lambda_{\theta_{t s}}-0.564 \ln \left(T u_{t s}\right)} & \left\{\lambda_{\theta t s} \leq 0\right\} \\
N=8.6 \times 10^{-4} e^{-0.564 \ln \left(T u_{t s}\right)-10 \sqrt{\lambda_{\theta_{t s}}}} & \left\{\lambda_{\theta t s}>0\right\}
\end{array}
$$

where $N$ is a non-dimensional spot production parameter proposed by Narasimha (1957), and defined as:

$$
N=\frac{n \sigma \theta^{3}}{\nu}
$$

Although the model of Solomon et al. (1996) was developed from experiments in attached flows, the pressure distributions upon which the model is based are similar 
to those which may cause laminar separation in turbomachines. This has allowed for realistic prediction of separation bubble transition using this model (e.g. Sanz \& Platzer, 1997; Müller et al., 2000). However, as will be discussed in Chapter 4, the model of Solomon et al. (1996) consistently under-predicts the streamwise rate of increase of intermittency, or transition rate, of the current experiments in attached flows. Poor prediction of the transition rate also resulted in separated flows, although this may be attributed to the fact that the model of Eqs. 2.17 and 2.21 was not originally intended to predict separation bubble transition. Modeling spot production rates are discussed further in Chapter 8 .

Johnson \& Ercan (1997) proposed an alternative approach for modeling the intermittency distribution in the transition zone, with a focus on conditions with elevated levels of free-stream turbulence $(T u>2 \%)$. The model does not assume a concentrated spot production, and bases the spot production rate on the near-wall and free-stream turbulence length scales in addition to the local free-stream turbulence intensity and pressure gradient. The model also includes new expressions for the spot propagation parameter, $\sigma$, and spreading angle, $\alpha$, although these differ from the predictions based on Eqs. 2.18 and 2.20 mostly for high adverse pressure gradients $\left(\lambda_{\theta}<-0.10\right)$. The model was validated through comparisons with experimental data published by Gostelow and his co-workers. Compared to the model of Solomon et al. (Eq. 2.17), prediction of the intermittency distribution was improved in the early and late stages of transition. 


\subsubsection{Separation Bubbles}

Since the transition process in separation bubbles normally occurs very rapidly, many early prediction models (e.g. Horton, 1968; Roberts, 1980) assumed a point transition. This allows for reasonable predictions in instances where the separated region consists of a small fraction of the airfoil or blade surface. However, in turbomachines, and particularly in low pressure turbines, the length of a separation bubble may extend up to $30 \%$ of the suction surface length of the blade before bursting or massive separation occurs (e.g. Volino, 2002-b). In such instances, the assumption of a point transition is not always adequate for the purposes of predicting aerodynamic losses on these blades.

There appears to be a substantial lack of effort in modeling the transition length in separation bubbles, in part due to the fact that the transition length tends to be shorter than in attached flows. In addition, since the location of reattachment generally corresponds closely to the location of transition completion, and the reattachment location is more relevant to the prediction of the downstream development of the turbulent boundary layer, the tendency has been to develop correlations for the reattachment location instead, such as the one proposed by Hatman \& Wang (1999):

$$
R e_{r}=1.0608 R e_{s}+34,890
$$

The experiments upon which Eq. 2.23 is based were conducted under low free-stream turbulence and steady mean flow conditions, and as a result, are not directly relevant 
to most turbomachinery applications. More recently Volino \& Hultgren (2001) and Yaras (2002) have studied the separation bubble under free-stream turbulence conditions more representative of gas turbine engines. A more recent model, which agrees with that of Hatman \& Wang at low turbulence levels, was proposed by Yaras (2002) to account for the effects of free-stream turbulence on the behaviour of short separation bubbles:

$$
R e_{r}=1.04 R e_{s}+8.05 \times 10^{4}\left(1-\tanh ^{3}\left(T F^{\prime}(\%)\right)\right)-2.0 \times 10^{4}
$$

where $T F^{\prime}=\max (T F(\%), 1 \%)$.

Because of the unsteady nature of the reattachment location, the uncertainty of the experimental data upon which these correlations are based is expected to be somewhat higher than for the locations of separation and transition inception. Additionally, interpreting the location of reattachment through the shape of the time-averaged velocity profile and/or a peak in turbulence intensity involves a high degree of subjectivity. It would therefore be useful to establish correlations for the intermittency distributions in the separated shear layer from which the end of the transition process can be deduced with higher precision. In the current study, and in the recent studies of Volino (2002-b), and Gostelow \& Thomas (2005), intermittency distributions in separation bubbles have been measured with sufficient streamwise resolution to allow the development of such models, as will be presented in Chapter 8 . 


\subsection{Additional Conditions Influencing Transition}

Without exception, the models described in the previous section have assumed that the only factors affecting transition are free-stream turbulence, pressure gradient, and Reynolds number. It is well known however that there are a number of additional conditions having a significant influence on the transition process, such as streamwise surface curvature, periodic flow unsteadiness, and surface roughness. These factors, and some of the important research efforts associated with each, are introduced in the following sections.

\subsubsection{Surface curvature}

The studies outlined up to this point have for the most part been concerned with the boundary layer developing over a flat surface. However, effects of streamwise surface curvature on the development of the boundary layer have also been noted independently of the effects of pressure distribution. Reynolds noted in 1884 that convex curvature, such as that on the suction surface of a turbine blade, provided a stabilizing influence on the boundary layer, whereas concave curvature, such as on the pressure surface of turbine blades, represented a destabilizing influence. These effects are related to the pressure and velocity gradients normal to the surface, as demonstrated in the following section.

For a fluid particle traveling along an equilibrium streamline, the pressure gradient normal to the surface provides the centripetal acceleration required for the particle to maintain its position relative to the solid boundary (i.e. $d P / d r=\rho v^{2} / r$ ). 


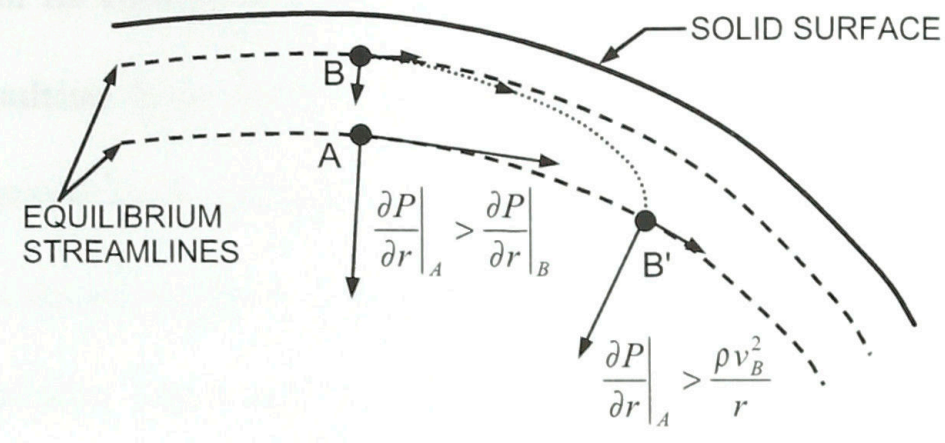

Figure 2.5: Destabilizing influence of concave curvature

However, a particle may be disturbed from its equilibrium flowpath, for example by free-stream turbulence fluctuations, disrupting the balance between the pressure gradient and centripetal acceleration. Whether the imbalance tends to restore the particle to its original state, or causes further motion away from its equilibrium position, depends on whether the surface is convex or concave. In the example of Fig. 2.5, two particles (A and B) are initially travelling along their respective equilibrium streamlines, represented by the dashed lines. Particle B has lower velocity than particle A, since its unperturbed position is closer to the solid boundary, and is therefore deeper in the boundary layer. To remain in equilibrium, the pressure gradient acting on particle $\mathrm{A}$ must be greater than that acting on particle $\mathrm{B}$ $\left(\left.\frac{d P}{d r}\right|_{A}>\left.\frac{d P}{d r}\right|_{B}\right)$. Now suppose a perturbation causes particle B to move from its equilibrium streamline to that initially occupied by particle A. For convenience, we may assume that the particle moves from $\mathrm{B}$ to $\mathrm{B}^{\prime}$ instantaneously, and therefore its momentum is unchanged. In the perturbed state $\left(\mathrm{B}^{\prime}\right)$, the pressure gradient acting normal to the surface is greater than that required for the particle to travel along this streamline $\left(d P / d r>\rho v^{2} / r\right)$, causing continued motion toward the centre of curvature, 
and away from its equilibrium position. Conversely, for convex curvature, the force imbalance resulting from disturbing a particle from its equilibrium path tends to restore the particle to its equilibrium flowpath. A similar analysis led Görtler (1940) to predict the development of longitudinal vortices in flows with concave curvature. These vortices (now called Görtler vortices) were later observed experimentally.

The effects of streamwise curvature typical of turbomachinery blades on the development of the boundary layer are generally believed to be much weaker than those of free-stream turbulence and pressure gradient. Thus, curvature effects have received less attention in the turbomachinery literature. Nonetheless, several recent studies have examined the effects of surface curvature on the transition process in the boundary layer, including effects of turbulence intensity (Kim et al., 1992), and streamwise pressure gradient (Wang \& Simon, 1987). Volino \& Simon (1995) reviewed the experimental data that were available at the time, and noted significant changes in turbulent spot production rates for flows over concave surfaces, but little effect on these parameters in the case of convex curvatures. The pressure distributions in the current study are similar to those prevailing on the suction (convex) side of low pressure turbines. Thus, the use of a flat surface, and exclusion of curvature effects in the modeling efforts presented in Chapter 8, is justified for the purposes of the present investigation.

\subsubsection{Periodic Unsteady and Wake-Induced Transition}

In recent years, discrepancies in the time-averaged heat transfer and aerodynamic losses have been observed in comparisons between cascade measurements 


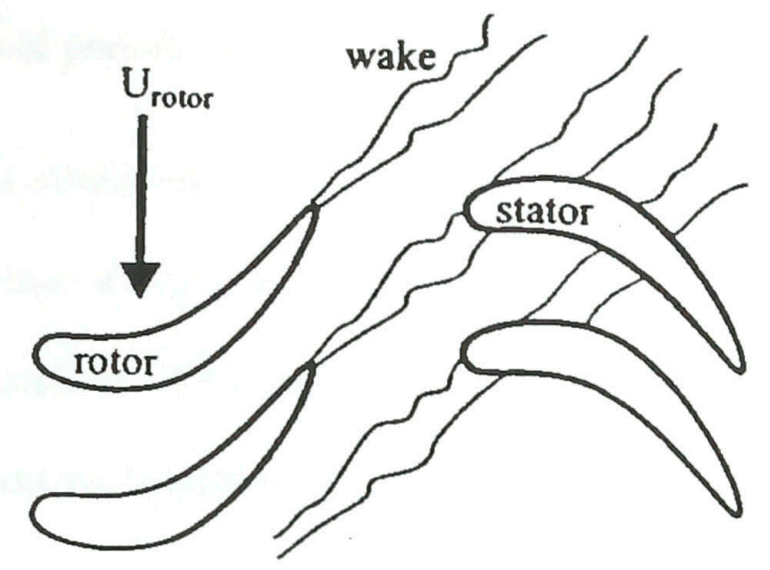

\section{Figure 2.6: Wake impingement in an axial turbine (from Ham et al., 2000)}

and tests conducted in more realistic gas turbine environments (Mayle, 1991). These discrepancies have been attributed to differences in the flow behaviour, including the transition process, caused by the periodic passing of the wakes shed by upstream components (Fig. 2.6). This phenomenon consists of two separate effects: a periodic fluctuation in the free-stream velocity, due to the momentum deficit in the wake, and a periodic disturbance imposed on the boundary layer as the wake eddies interact with the boundary layer.

Recently, research efforts to predict the transition process in turbomachinery applications have included the effects of periodic-unsteady free-stream flow and boundary layer-wake interactions. Pfeil et al. (1983) noted that the flow in the boundary layer affected by the wake became turbulent with each wake passing, and that the length of the turbulent "strip" grew as the wake impingement occurred further downstream along the blade surface. In between the passing of these wakes, the flow was seen to exhibit a region of natural transition, similar to that which would 
be seen in the absence of periodic-unsteadiness.

The more recent studies of Addison \& Hodson (1990-a,--b) and Hodson (1991) have documented the time-averaged and ensemble-averaged shear stresses on both the suction and pressure surfaces of turbine blades. Schobeiri \& Radke (1994) studied the effects of wake induced transition on a concave curved plate, and Solomon \& Walker (1995) have documented ensemble-averaged shear stress and intermittency on the surface of compressor blades. Although these studies have provided useful insight into the behaviour of the boundary layer in instances of wake induced transition, the effects of periodic free-stream unsteadiness and wake induced transition on separation bubble transition remain an area of active research. For example, Dong \& Cumpsty (1990-a,-b), Kaszeta et al. (2001), Schobeiri et al. (1995) and Stieger \& Hodson (2004) noted that the periodic passing of wakes caused the suppression and subsequent reappearance of the separation bubble. Lou \& Hourmouziadis (2000) used rotating flaps downstream of the test surface to generate periodic free-stream velocity fluctuations, an experimental setup that was also used in the present research (Chapter 5). Although periodic fluctuations in the separation bubble were noted by Lou \& Hourmouziadis (2000), the complete suppression of the separation bubble noted by Dong \& Cumpsty (1990-a) and Stieger \& Hodson (2004) was not observed. The results of the study of Lou \& Hourmouziadis (2000) suggest the effects of wake eddies to be more significant than those of the free-stream velocity fluctuations. However, the above-noted studies were performed with low free-stream turbulence levels. The response of a separation bubble to periodic free-stream velocity fluctuations, as in the experiments of Lou \& Hourmouziadis (2000), but with a range of free-stream 
turbulence levels, constitutes part of the present research (Chapter 5).

\subsubsection{Surface Roughness}

Surface imperfections that gradually develop on the blades of gas turbine engines can result in substantial reductions in stage aerodynamic efficiency (Leipold et al., 2000; Kind et al., 1998; Boynton et al., 1992; Suder et al., 1995; Boyle \& Senyitko, 2003), and also potentially in thermal damage to the blades due to changes in the surface heat transfer rates (Bons \& McClain, 2003; Blair, 1994; Pinson \& Wang, 1997; Wang \& Rice, 2003). Surface heat transfer rates may also be affected by changes in the surface material properties when the roughness is due to hydrocarbon deposits, or when a protective coating is eroded. On turbine blades, the source of surface imperfections is typically hydrocarbon deposits and/or pitting and spalling damage caused by the impact of small particles. The level of surface roughness on a gas-turbine blade, which can vary in height from $2 \mu \mathrm{m}$ to $160 \mu \mathrm{m}$ (Bons et al., 2001; Taylor, 1990), depends on the location of the blade within the engine, and the length of time it has been in service. The distribution of roughness over the blade surface is typically non-uniform, with significant variations in both the streamwise and spanwise directions (Bons et al., 2001; Taylor, 1990). For example, the roughness level at the leading edge of a high-pressure-turbine stator blade (Fig. 2.7-a) is noted to be of larger scale than that observed near the suction peak of a low-pressure turbine rotor blade (Fig. 2.7-b) from the same engine. Closer proximity to the combustor and more rapid accumulation of particles causing roughness in stagnation regions are responsible for the rougher surface conditions noted near the leading edge of the 


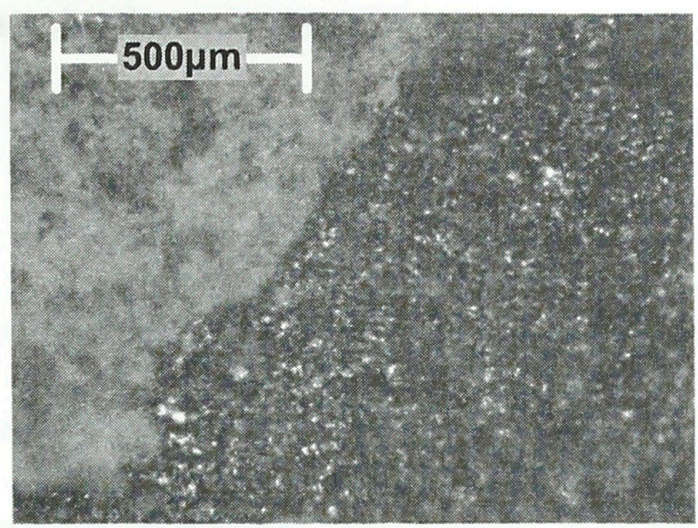

(a) HP Nozzle LE

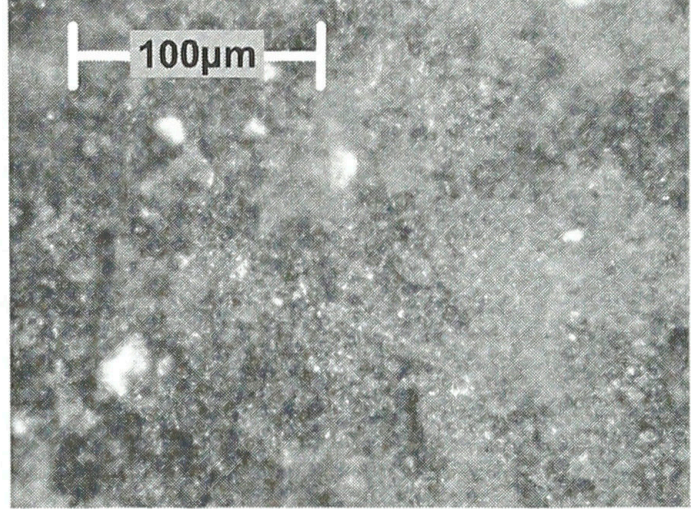

(b) LPT Suction Peak

Figure 2.7: Surface roughness levels at the leading edge of an HP turbine nozzle (a) and near the suction peak of an LP turbine blade (b)

NOTE: Magnification is higher in (b)

stator blade.

Understanding the effects of surface roughness is essential for predicting the performance of turbines, and thus optimizing blade designs for maximum efficiency and component life. One means by which surface roughness affects the aerodynamics and heat transfer rates is through alteration of the boundary layer transition process. Many published studies have examined the effects of isolated surface disturbances on the transition process (e.g. Würz et al., 2003; Kachanov, 2000; Lang et al., 2004). These studies have reported increased receptivity of the boundary layer to disturbances in the presence of surface roughness, and increased amplification rates of T-S waves. These trends were noted to be applicable to attached (Würz et al., 2003; Kachanov, 2000) as well as separated flow transition (Lang et al., 2004). However, these studies do not reproduce the conditions of non-uniform streamwise pressure gradients, elevated free-stream turbulence and periodic unsteadiness that are typical in turbomachinery blade passages. Thus, while such studies offer considerable insight 
into the flow physics of boundary layer transition over rough surfaces, they are not of direct use in engineering. Several studies (e.g. Kerho \& Bragg, 1997; Cummings \& Bragg, 1996) have examined the effects of large scale leading-edge roughness on boundary layer transition over aircraft airfoils. However, the intent of such studies was to simulate the effects of leading edge icing on aircraft wings, and the roughness patterns considered makes them less applicable to turbomachinery applications.

\subsubsection{Roughness Height}

The presence of sufficiently large surface roughness is known to "trip" the laminar boundary layer, after which a very rapid transition process typically takes place (e.g. Kerho \& Bragg, 1997; Gibbings et al., 1986-a,--b,-c; Klebanoff \& Tidstrom, 1982). According to the roughness measurements compiled by Bons et al. (2001), in the mid-span/mid-chord region of the suction side of in-service turbine blades, the RMS roughness height varies between approximately 2 and $9 \mu \mathrm{m}$. For typical ranges of Reynolds numbers and pressure gradients, this level of surface roughness tends to be insufficient to cause bypass transition. It is however expected that the disturbances created by the roughness elements would tend to accelerate the natural transition process. For example, the location of transition inception in attached boundary layers has been noted to move progressively upstream with increases in surface roughness (e.g. Pinson \& Wang, 1997; Gibbings \& Al-Shukri, 1997; Kerho \& Bragg, 1997). Similar trends have also recently been observed for transition in separation bubbles (Volino \& Bohl, 2004). 


\subsubsection{Roughness Geometry}

For a given surface roughness height, the geometry of the roughness pattern may also have a significant effect on how the boundary layer development is affected by the surface conditions. It was suggested by Morris (1955) that the effect of roughness on the boundary layer occurs primarily through vorticity and turbulence shed in the wakes of roughness elements. The influence of this wake turbulence on the surrounding flow, and the extent by which neighbouring wakes interact, are affected by the shape, size, and spacing of the roughness elements. The effects of these features of roughness patterns on turbulent boundary layers have been studied extensively for both deterministic (e.g. Waigh \& Kind, 1998; Dirling, 1973; Dvorak, 1969), and random (e.g. Bons, 2002; Belnap et al., 2002; Sigal \& Danberg, 1990) roughness. The recent experiments of Stripf et al. (2005) over deterministic rough surfaces suggest the roughness geometry to be less influential in transitional flows than in fully turbulent boundary layers. Nonetheless, movement of the location of transition inception with variations in roughness spacing is evident in their experimental data, with the effect of spacing becoming less pronounced at the highest Reynolds numbers and turbulence levels tested.

\subsubsection{Non-Uniform Roughness Height Distribution}

As noted earlier, the measurements of Bons et al. (2001) and Taylor (1990) indicate the distribution of roughness over turbine blade surfaces to be highly nonuniform. Based on these measurements, Pinson \& Wang (1997) examined the effects of streamwise variation in roughness conditions in order to better simulate 
the roughness patterns typical of gas-turbine blades. They concluded that the abrupt change in roughness height between the leading edge and the remainder of the surface is more influential on attached flow transition than the downstream roughness level. The combined influence of surface roughness and free-stream turbulence has been studied recently by Wang \& Rice (2003). This study demonstrated that the process of attached flow boundary layer transition remains sensitive to surface roughness at elevated levels of free-stream turbulence, although no models to predict these effects were proposed.

The experiments presented in Chapter 6 were performed to clarify the combined effects of surface roughness and free-stream turbulence on boundary layer transition, for conditions typical of low pressure turbines. These measurements form the basis for a transition inception model for separation bubbles presented in Chapter 8.

\subsection{Numerical Simulation}

Advances in computing power have allowed more refined numerical solutions of the Navier-Stokes equations such that they can be used as numerical wind tunnels. The most refined of such studies, known as direct numerical simulations (DNS), resolve the flow field down to the smallest scale of turbulence. While this technique has been successfully used in studying the transition process in attached (e.g. Rai \& Moin, 1993; Singer \& Joslin, 1994) and separated (e.g. Alam \& Sandham, 2000; Spalart \& Strelets, 2000; Maucher et al., 1999) boundary layers, the substantial computing 
requirements, proportional to the $3^{\text {rd }}$ power of flow Reynolds number (Pope, 2000), have limited such investigations to simplified computational domains at relatively low flow Reynolds numbers. To circumvent this limitation, one can resort to large-eddy simulations (LES). In LES, the spatial and temporal discretization of the flow provide adequate resolution only for the larger turbulence eddies. This is accomplished by filtering the Navier-Stokes equations, with the spatial grid typically serving as the filter. The lack of full resolution of the turbulence spectrum with this technique requires the use of a sub-grid scale (SGS) turbulence model. As a minimum, the SGS model is to provide the correct amount of energy transfer from the resolved scales of turbulence to the unresolved range (forward scatter). In transitional flows, the energy transfer from smaller to larger scales of turbulence (backscatter) has been argued to be important (Dubois \& Bouchon, 1998), which adds to the demand from the sub-grid scale model in these flows.

Many investigators have used large eddy simulations to predict transition in moderately complex flows (e.g. Huai et al., 1997; Ducros et al., 1996), similar to those encountered in the experimental phases of the current project. In addition, LES has been used by Ham et al. (2000) to model wake-induced transition over a flat plate, and by Cabot (1998) to simulate a separated boundary layer.

In typical large-eddy simulations of boundary layers, the scale of the resolved turbulence is typically of the order of 1000 wall units (Spalart, 2000). Due to the substantial effects the SGS models may have on the development of the transition process, a notable portion of published studies on LES of transitional flows have 
focused on the evaluation of these models (e.g. Rodi et al., 1997; Lesieur \& Métais, 1996). Despite the considerable progress in this research field, a model that allows the reliable use of LES to explore the physics of transition is yet to be developed. In the interim, many have resorted to resolving as much of the turbulence as possible (e.g. Yang \& Voke, 2001; Ducros et al., 1996), thereby minimizing the effects of the sub-grid scale model on the LES results.

To overcome the limitations of current measurement techniques, the experimental results of the current study are complemented by simulation of one of the test cases using LES. In the current work, the spatial and temporal grid of the solution is refined to the extent that the performance of the sub-grid scale model is of minor importance. This approach requires a considerably finer discretization of the computational domain than the Reynolds averaged Navier-Stokes (RANS) simulations with one- or two-equation turbulence models that are currently the standard in the turbomachinery industry. As a result, the computational effort is too great for this method to be of practical use in predicting the complex, threedimensional flows in turbomachines. This portion of the study is therefore not intended as an evaluation of the capabilities of LES in an industrial setting, but instead offers a unique perspective of the flow dynamics in a transitional separation bubble. 


\section{Chapter 3}

\section{Experimental Setup and Data Analysis}

\subsection{Test Section Configuration}

The test section used for the current study is installed in the closed-circuit wind tunnel in room 3224 of the Mackenzie Engineering building at Carleton University. The overall dimensions of the current test section are $762 \mathrm{~mm}, 508 \mathrm{~mm}$ and $1830 \mathrm{~mm}$ in width, height and length, respectively. The test section had been commissioned, and used extensively in transition studies, prior to the experiments of the present study (e.g. Yaras 2001, 2002). Streamwise pressure distributions are imposed on the flat test surface by adjusting the geometry of the flexible ceiling of the test section (Fig. 3.1). During the course of the study, four different ceiling geometries were used, and the resulting pressure distributions are designated $C_{P 1}$ through $C_{P 4}$. The geometric settings for each of these configurations are presented in Appendix A.

The test plate is supported by four vertically mounted threaded rods contained 


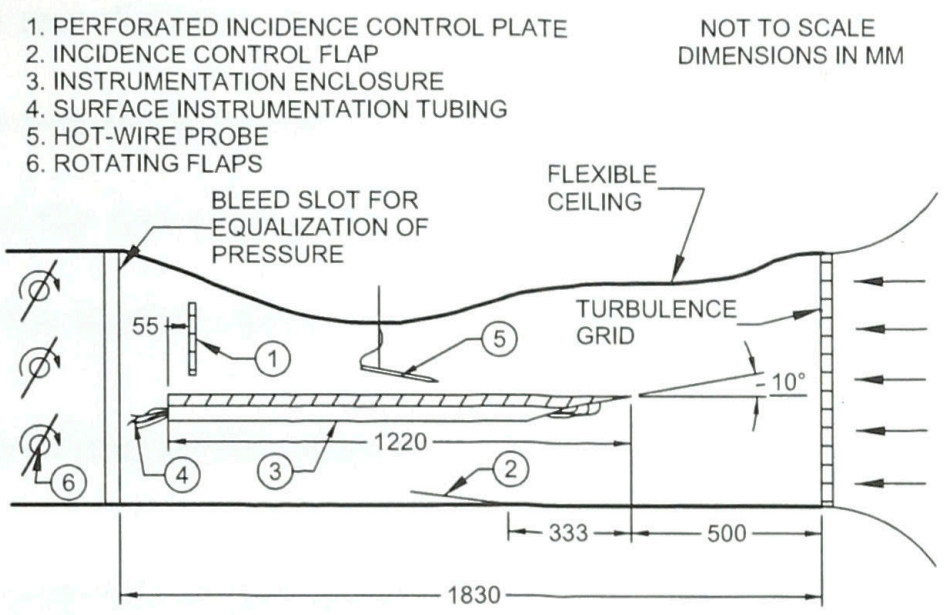

Figure 3.1: Schematic of the wind tunnel test section

in U-channels placed on the sides of the test section. The support assembly is located outside of the test section, resulting in aerodynamically clean inner side walls. The threaded rods allow adjustment of the pitch angle, and position relative to the ceiling, of the test surface. The boundary layer is tripped at the upstream end of the flexible ceiling to ensure a turbulent state. Based on flow visualization experiments, this measure is sufficient to prevent flow separation from the ceiling.

The present testing arrangement has been used by a number of research groups for transition measurements (e.g. Gaster, 1967; Passchier, 1997; Volino \& Hultgren, 2001; Lou \& Hourmouziadis, 2000). It allows for investigation of the transition process in the absence of surface curvature, and is more suitable for accurate positioning of measurement probes than airfoil geometries.

\subsubsection{Control of Flow Incidence}

The flow incidence at the leading edge of the test surface is controlled by partial blockage of the flow passing over the test plate with a 22 -gauge perforated steel ( $51 \%$ 
open area; $4.76 \mathrm{~mm}$ diameter holes on $6.35 \mathrm{~mm}$ staggered centres) installed $55 \mathrm{~mm}$ upstream of the test-plate trailing edge, as well as through the use of a $255 \mathrm{~mm}$ long flap mounted on the test section floor, the leading edge of which is located $333 \mathrm{~mm}$ downstream of the leading edge of the test plate (Fig. 3.1).

\subsubsection{Two-Dimensionality of the Flow}

For the purposes of the present study, it is essential that any effects of flow three dimensionality on the transition process be minimized. In the present test section, one potential source of such three dimensionality is spanwise variation in flow incidence at the test plate leading edge. Spanwise uniformity of flow incidence was confirmed through 7 -hole pressure probe measurements $30 \mathrm{~mm}$ upstream of the plate leading edge.

Another potential source of flow three-dimensionality is the secondary flow that may develop along the sidewalls due to the presence of cross-stream pressure gradients. In the smooth surface experiments, surface static pressure measurements taken at three spanwise locations were in excellent agreement with each other, thus confirming two-dimensionality of the flow. An example of the spanwise uniformity of surface static pressure coefficient $\left(C_{P}=\frac{P-P_{\text {ref }}}{\frac{1}{2} \rho U_{r e f}^{2}}\right)$ is shown in Fig. 3.2.

\subsubsection{Test Surfaces}

\subsubsection{Smooth Surface}

The test plate used for the smooth surface measurements is of $1220 \mathrm{~mm}$ length, $756 \mathrm{~mm}$ width, and $25.4 \mathrm{~mm}$ thickness, and is made of CHART-MIC-6 aluminium. 


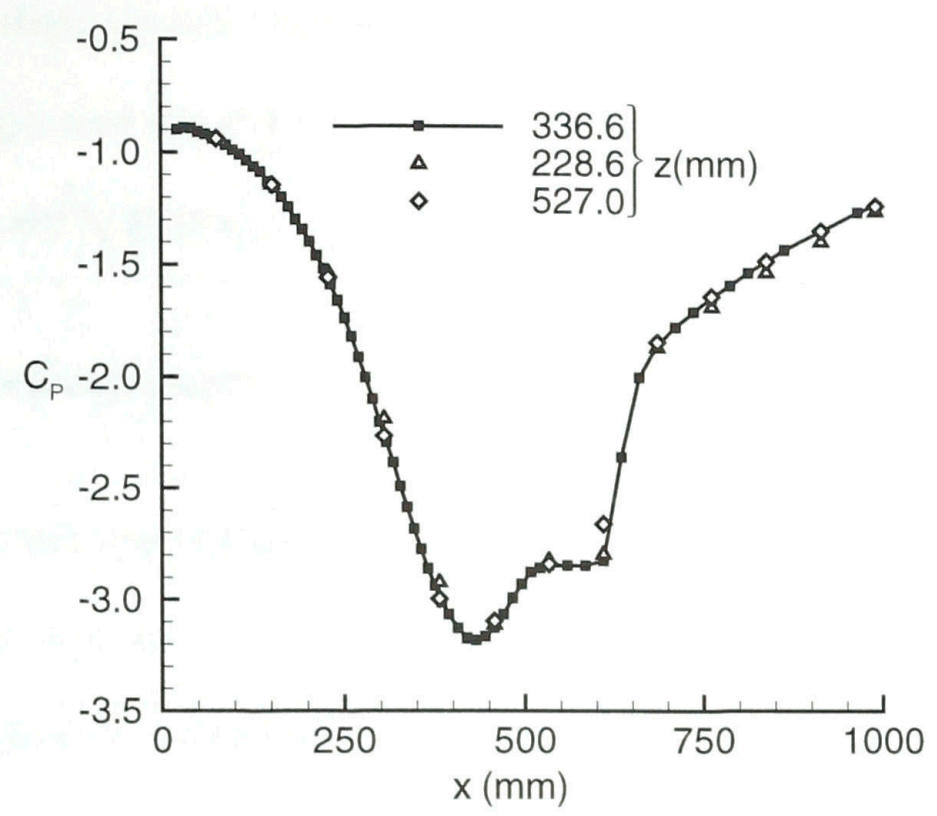

Figure 3.2: Surface pressure measurements at three spanwise locations $\left(R e_{L}=350,000\right.$; $T u_{r e f}=0.5 \% ; C_{P 1}$ pressure distribution)

The root-mean-square roughness height of this test surface is less than $0.7 \mu \mathrm{m}$ and the flatness is within $0.12 \mathrm{~mm}$ over the length of the plate. This is the only test surface with static pressure taps, and has an instrumentation enclosure to contain the tubing of these taps, as shown in Fig. 3.1. The leading edge is machined to a knife-edge of $10^{\circ}$ from the lower side of the plate. The tip of this knife-edge is of elliptic geometry, with major and minor axis dimensions of $2.54 \mathrm{~mm}$ and $0.5 \mathrm{~mm}$ for the measurements conducted under steady mean flow conditions (Chapter 4). Such a sharp leading edge was chosen to minimize curvature and acceleration effects on the initial development of the boundary layer. An elliptic geometry was chosen due to the greater margin of incidence without boundary layer separation compared to double circular arc forms (Davis, 1980).

For the experiments conducted under periodic-unsteady free-stream conditions 
(Chapter 5), slight periodic fluctuations in flow incidence are likely. Thus, a leading edge with larger axis dimensions $(15.9 \mathrm{~mm}$ and $3.2 \mathrm{~mm})$ was chosen to prevent flow separation at the leading edge.

\subsubsection{Rough Surfaces}

The rough test surfaces were constructed by bonding materials of desired roughness patterns onto a $25.4 \mathrm{~mm}$-thick MDF board. Throughout this document, the rough surfaces are identified by their value of root-mean-square roughness height $\left(k_{r m s}\right)$. These roughness layers consist of two countertop laminates $\left(k_{r m s}=31 \mu \mathrm{m}\right.$, $\left.k_{r m s}=33 \mu \mathrm{m}\right)$, vinyl flooring material $\left(k_{r m s}=48 \mu \mathrm{m}\right)$, tar paper $\left(k_{r m s}=53 \mu \mathrm{m}\right)$, fine asphalt shingle $\left(k_{r m s}=107 \mu \mathrm{m}\right)$, and coarse asphalt shingle $\left(k_{r m s}=185 \mu \mathrm{m}\right)$ surfaces. The leading edge of these rough test-surfaces consists of a $50.8 \mathrm{~mm}$ long aluminium section with a smooth surface, mounted onto the test plate such that its top surface is aligned with the peaks of the roughness elements. As with the smooth plate, the leading edge attachment is machined to a knife-edge from its lower side. However, the angle of the knife-edge was increased to $15^{\circ}$ to minimize the distance between the leading edge and the beginning of the rough surface. Its tip geometry is elliptic with the same major and minor axis dimensions as for the smooth test plate used in the periodic-unsteady flow measurements (i.e. $15.9 \mathrm{~mm}$ and $3.2 \mathrm{~mm}$ ).

The geometries of the rough surfaces were measured through 3-D laser triangulation scans ${ }^{1}$. Because of the different roughness properties of each of the

\footnotetext{
${ }^{1}$ Surface scans for the $k_{r m s}=31,33,53,107$, and $185 \mu \mathrm{m}$ surfaces were performed by LaserVision Technologies, Inc. of Maple Park MN. The measurements for the $k_{r m s}=31,33,107$ and $185 \mu \mathrm{m}$ surfaces were repeated by XYZ-RGB, Inc. of Ottawa, ON, along with several additional surfaces,
} 
Table 3.1: Surface scan areas and resolutions

\begin{tabular}{|l|c|c|}
\hline Surface & $\begin{array}{c}\text { Scan Area } \\
(\mathrm{mm} \times \mathrm{mm})\end{array}$ & $\begin{array}{c}\text { Scan Resolution } \\
(\mu \mathrm{m})\end{array}$ \\
\hline Smooth Surface & N/A & N/A \\
\hline Blue Laminate & $10 \times 10$ & $40 \mu \mathrm{m}$ \\
\hline White Laminate & $10 \times 10$ & $40 \mu \mathrm{m}$ \\
\hline Vinyl & $30 \times 32$ & $100 \mu \mathrm{m}$ \\
\hline Tar Paper & $8 \times 8$ & $20 \mu \mathrm{m}$ \\
\hline Fine Shingle & $8 \times 8$ & $25 \mu \mathrm{m}$ \\
\hline Coarse Shingle & $12 \times 12$ & $40 \mu \mathrm{m}$ \\
\hline
\end{tabular}

surfaces, the scan area and scanning resolution is adjusted from one surface to the next. These scanning areas and resolutions are summarized in Table 3.1. The statistical roughness parameters calculated from the scan data are defined and given in Chapter 6, where the experimental results for these rough surfaces are discussed.

\subsubsection{Variation of Free-stream Turbulence}

The level of free-stream turbulence in the test section is adjusted by placing a perforated plate in the flow path at the test-section inlet. The plates, made of 3.18 mm-thick aluminum, are designed to cover the whole cross-section and the openings in the plates consist of non-staggered, square holes. The specifics of the perforated plates are summarized in Table 3.2.

With the exception of perforated plate \#4, the plates are placed at the inlet plane of the test section which is $500 \mathrm{~mm}$ upstream of the test-plate leading edge. For perforated plate \#4, this distance is increased to $600 \mathrm{~mm}$. Based on single

including the $k_{r m s}=48 \mu \mathrm{m}$ surface. The results of the repeated surface scans were substantially the same as the original measurements $( \pm 6 \%)$. To avoid inconsistency with publications that appeared prior to the repeated measurements, for surfaces where duplicate measurements exist, quantities based on the scan data of LaserVision Technologies are quoted in the text. 
Table 3.2: Turbulence generator specifications

\begin{tabular}{|l|c|c|c|c|c|c|}
\hline & \multicolumn{7}{|c|}{ Perforated Plate \# } \\
\cline { 2 - 7 } & $0^{*}$ & 1 & 2 & 3 & 4 & 5 \\
\hline Square Hole Size (mm) & - & 12.7 & 25.4 & 12.7 & 25.4 & 50.8 \\
\hline Hole Spacing (mm) & - & 15.9 & 31.8 & 18.0 & 35.9 & 63.5 \\
\hline Open-Area Ratio (\%) & 100 & 64 & 64 & 50 & 50 & 64 \\
\hline Range of Tu ref $\left._{\text {ryyyyy}} \%\right)$ & $0.5-0.9$ & $2.2-2.5$ & $4.3-4.6$ & $3.7-3.9$ & $6.1-6.8$ & $8.9-9.0$ \\
\hline
\end{tabular}

* No grid installed

sensor hot-wire centreline traverses $10 \mathrm{~mm}$ upstream of the leading edge of the test plate, covering a height from the level of the test plate to the ceiling, these turbulence generator locations have been found to produce nearly uniform mean-flow and turbulence distribution at the test plate leading edge, with variations of less than $5 \%$ in both $\bar{U}$ and $T u_{r e f}$, as shown in Fig. 3.3-a. The uniformity of free-stream turbulence is noted to be independent of the Reynolds number, pressure distribution, and surface roughness. With the exception of perforated plate \#4, the distribution of turbulence length scale is also noted to be reasonably uniform up to a point $100 \mathrm{~mm}$ above the test surface, as shown in Fig. 3.3-b. This observation is consistent with about 10 mesh spacings that is suggested to be the required distance for achieving homogeneous turbulent flow behind a grid (Roach, 1997). The turbulence was less uniform with perforated plate $\# 4$, which is recessed into the contraction of the wind tunnel by $100 \mathrm{~mm}$. The steps at the top and bottom of the inlet to the test section, created by this arrangement, cause an increase in turbulence intensity, and especially in the length scale, as the probe is moved away from the surface (Fig. 3.3).

In addition to the reference intensity $\left(T u_{r e f}\right)$, and the free-stream turbulence intensity at the transition inception location $\left(T u_{t s}\right)$ documented in the chapters of 


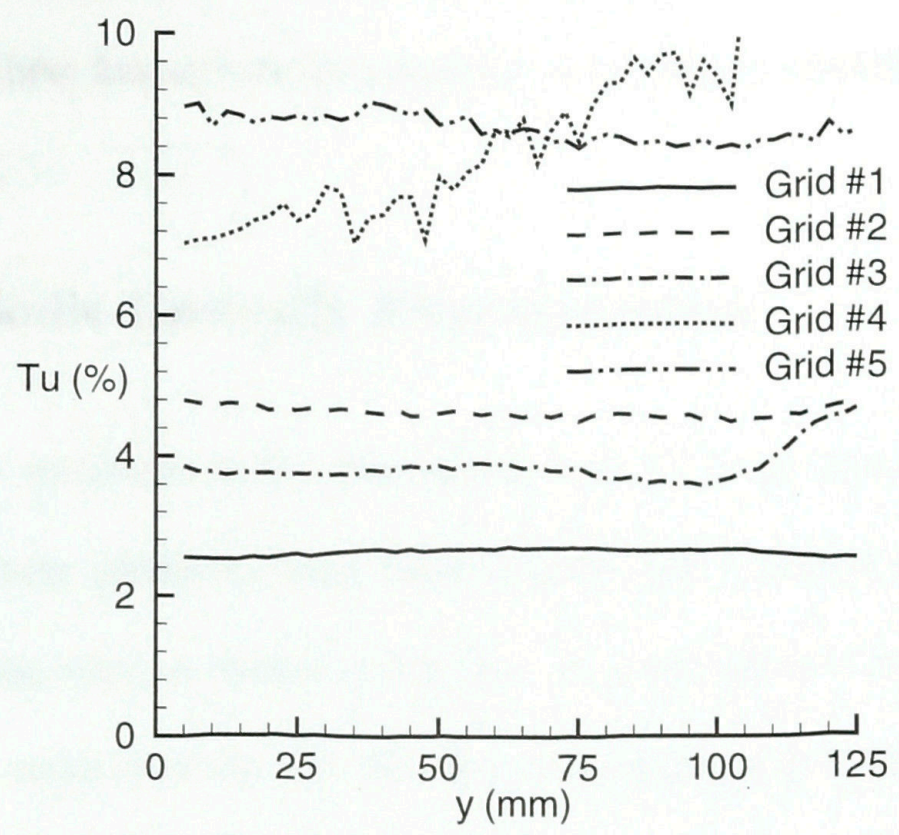

(a) Turbulence intensity $\left(T u_{r e f}\right)$

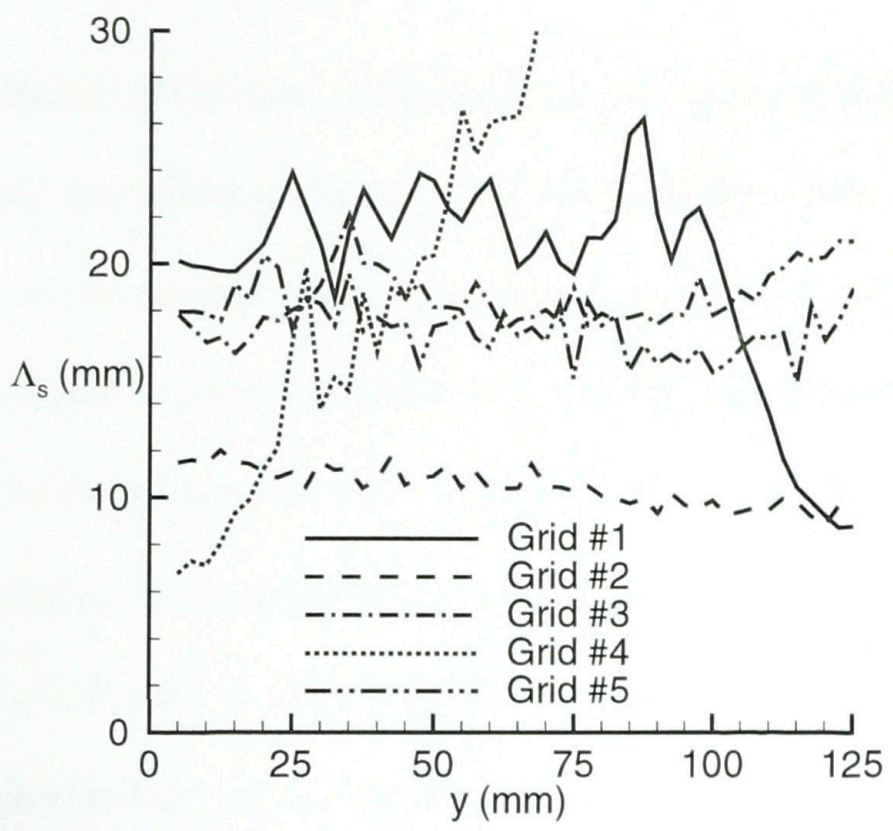

(b) Integral length scale $\left(\Lambda_{s}\right)$

Figure 3.3: Surface-normal variation of reference freestream turbulence $\left(\operatorname{Re}_{L}=470,000\right)$ 
the thesis, the streamwise variation of free-stream turbulence intensity is often of importance in characterizing certain trends related to the transition process. For completeness, these free-stream distributions of turbulence intensity are documented in Appendix B.

\subsubsection{Periodic-Unsteady Flow Generator}

Periodic variations in the free-stream velocity in the test section (discussed in Chapter 5) were produced using three rotating flaps, located downstream of the test plate trailing edge, as shown in Fig. 3.1. Precise control of the rotational speed of the flaps is maintained by the use of a variable-frequency $\mathrm{AC}$ motor drive. In addition, the rotating flaps are driven using cogged belts to eliminate any variation in rotational speed due to slippage.

The amplitude of the flow oscillations can be varied by changing the width of the rotating flaps, thus altering the extent of flow blockage when the flaps are in the closed position. In the present setup, flaps of $114.3 \mathrm{~mm}$ width are used, mounted on three 28.6 mm-diameter axles, resulting in a blockage of approximately $61 \%$ of the flow area when the flaps are in the fully closed position, as compared to the fully open position. The spacing of the three axles are chosen such that the open areas at the top and bottom of the test section, and those between the flaps, are of the same size when the flaps are in their maximum blockage position. The resulting peak-to-peak amplitude of the flow oscillations measured $10 \mathrm{~mm}$ upstream of the leading edge of the test plate varies approximately from $3 \%$ to $30 \%$, depending on the frequency of oscillation, and the flow Reynolds number. For comparison, typical values of the 
velocity deficit in the wakes of passing turbomachinery blades, as they are about to impinge on the downstream blades, range from approximately $8 \%$ to $15 \%$ of the freestream velocity (e.g Dong \& Cumpsty, 1990-b; Schobeiri \& Radke, 1994). For the baseline steady-flow measurements of Chapter 5, the flaps were set to their minimum blockage position.

It should be noted that, unlike wake-generated periodic flow oscillations, the velocity fluctuations produced in the present experiments decay in the upstream, rather than in the downstream direction. However, the rate of decay within the region of interest in this study was measured to be minimal. The difference in normalized amplitude of the velocity fluctuations between the leading edge of the test plate and the end of the transition zone was less than $5 \%$ for all test cases.

\subsection{Instrumentation}

\subsubsection{Surface Instrumentation}

The smooth test surface is instrumented with three streamwise rows of pressure taps of $0.635 \mathrm{~mm}$-diameter. The primary row of pressure taps is located at $z=337 \mathrm{~mm}$ (41 $\mathrm{mm}$ from the centreline) and consists of 40 taps of $9.5 \mathrm{~mm}$ (3/8 in.) spacing, starting $22 \mathrm{~mm}$ from the leading edge, followed by 11 taps of $12.7 \mathrm{~mm}$ (1/2 in.) spacing, 11 taps of $25.4 \mathrm{~mm}$ (1 in.) spacing, and 7 taps of $50.8 \mathrm{~mm}$ (2 in) spacing. This row is placed a small distance off the centreline to ensure that the hot-wire traverses at the plate centreline involve a boundary layer that is not affected by the presence of pressure taps in any way. The side rows, located at $z=229 \mathrm{~mm}$ 
and $z=527 \mathrm{~mm}$ consist of 15 taps of $76.2 \mathrm{~mm}$ (3 in.) spacing starting at $76.2 \mathrm{~mm}$ (3 in.) from the leading edge. The primary row near the centre of the plate is intended for quantifying the streamwise pressure distribution imposed on the boundary layer developing on the test plate surface, whereas the side rows assist in the verification of the two dimensionality of this boundary layer. Tygon ${ }^{\mathrm{TM}}$ tubing connected to these pressure taps is contained in a sheet-metal enclosure (Fig. 3.1) to ensure that the flow below the test plate experiences a uniform resistance across the span of the test section. A portion of the tubing connected to the pressure taps in close vicinity of the test plate leading edge remain outside the enclosure. Smaller diameter tubing (4 mm OD) is used in these cases to minimize their influence on the flow field at the leading edge.

\subsubsection{Hot-Wire Measurements}

A hot-wire probe with a single sensor of $5 \mu \mathrm{m}$ diameter and $1.3 \mathrm{~mm}$ length is traversed across the boundary layer at several streamwise positions along the centreline of the test plate. The hot-wire probe is calibrated specifically for the velocity range encountered in the present measurements (0 to $18 \mathrm{~m} / \mathrm{s}$ ). A polynomial curve-fit (typically $9^{\text {th }}$-order) is used to obtain the calibration coefficients, an example of which is shown in Fig. 3.4. Flow temperature inside the wind tunnel is measured in conjunction with the hot-wire signals to allow for compensation of temperature effects. Slight drifts in the hot-wire anemometer signal-conditioning circuitry is the main source of uncertainty in the measured velocity. To minimize the effect of this drift, the calibration curve was checked frequently, usually after boundary layer traverses 


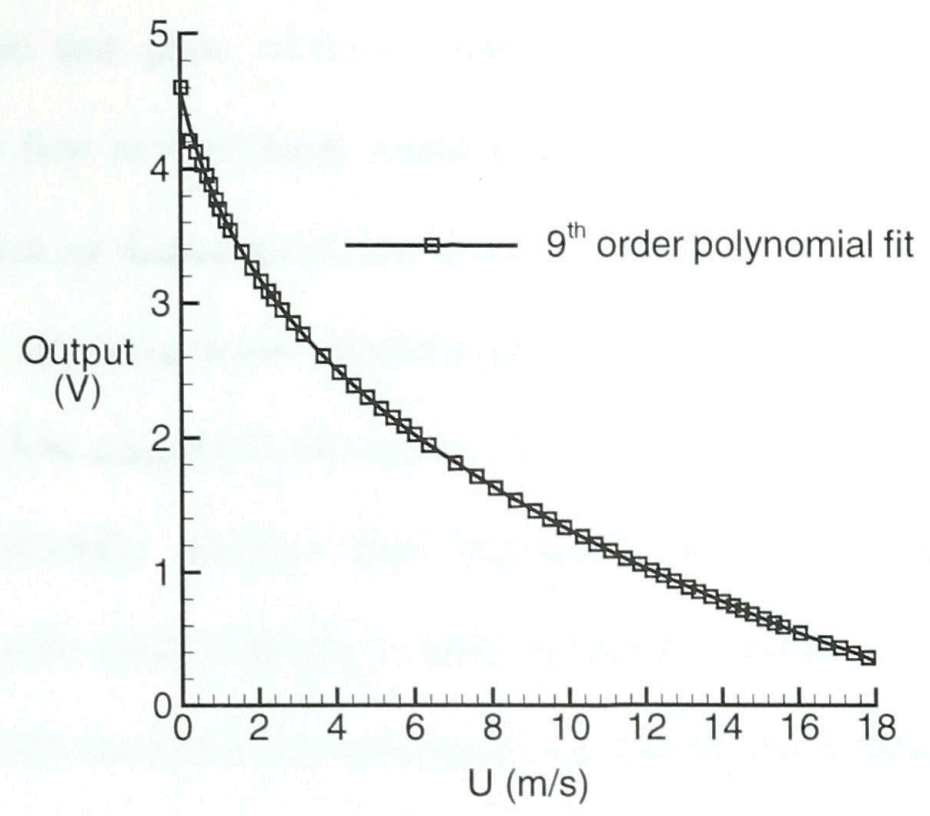

Figure 3.4: Sample hot-wire calibration curve

at two to three streamwise positions for multiple Reynolds numbers. The resultant uncertainty in the velocity measurements is estimated to be $\pm 2 \%$ of the local velocity, except for the relatively small velocity magnitudes $(<0.5 \mathrm{~m} / \mathrm{s})$ encountered very close to the surface, for which the uncertainty is judged to be closer to $\pm 4 \%$.

The probe stem supporting the hot-wire is suspended vertically through a longitudinal slot located at the centre of the flexible ceiling. The portion of this slot not occupied by the probe stem is covered with a thin packing tape from the inside. Traverse of the probe in the streamwise and vertical directions is performed using a precision traversing system.

The intrusive nature of the probe may be a concern when measuring the flow development in a small separation bubble. To minimize flow disturbances 
caused by the presence of the probe, the probe support is mounted at 10 degrees relative to the test plate surface. Additionally, single hot-wire probes cannot detect reverse flow in their basic configuration, which affects the values of integral parameters such as displacement and momentum thickness if the recirculation zone is included in the integration. Preliminary trials confirmed this effect to be small owing to the low magnitude of velocity in the recirculation region $(<5 \%$ of the free-stream velocity). Despite these limitations of single hot-wire anemometers, comparisons with results obtained using multi-wire probes (e.g. Passchier, 1997) and non-intrusive measurement techniques (e.g. Brendel \& Mueller, 1987) have been favourable. Furthermore, the locations of transition inception detected by the single hot-wire have been found to be in excellent agreement with those deduced from lasertwo-focus measurements in the present test section.

The number of measurement locations in each cross-stream traverse across the boundary layer varies between 25 near the leading edge of the test surface and about 80 further downstream, in the transition and/or separation regions. The streamwise spacing of the hot-wire traverses is normally chosen to be $50 \mathrm{~mm}$, except within the expected transition region where the spacing is set to $25 \mathrm{~mm}$, and in a few cases $12.5 \mathrm{~mm}$. In the test cases where a separation bubble is present, flow separation typically occurs approximately $500 \mathrm{~mm}$ downstream of the leading edge, and the distance between the time-averaged separation and reattachment locations varies between $50 \mathrm{~mm}$ and $125 \mathrm{~mm}$. Thus, the separation bubbles in the current study are resolved by two to five cross-stream traverses. 
In addition to the cross-stream traverses described above, for the test cases in which a separation bubble is present, traverses were performed in the streamwise direction from $x=475 \mathrm{~mm}$ to $x=599 \mathrm{~mm}$ in $2.0 \mathrm{~mm}$ intervals. The purpose of these streamwise traverses is to resolve the transition inception and completion locations, and growth rates of disturbances, with greater precision than is possible using the more sparsely spaced cross-stream traverses. For reference, the boundary layer thickness at the separation point is typically about $5 \mathrm{~mm}$, and the maximum thickness of the separation bubble is typically less than $8 \mathrm{~mm}$. To avoid clutter in the figures, only the intermittency distributions at $y=1.25 \mathrm{~mm}$ will be presented in this document, for test cases in which a separation bubble is present. However, because the intermittency distributions obtained from traverses at different elevations from the test surface $(1.25,2.0,4.0,6.0$, and $8.0 \mathrm{~mm})$ are in good agreement for all test cases, basing the intermittency distributions on measurements at a single elevation is justified. Although the spatial resolution of the streamwise traverses is $2 \mathrm{~mm}$, due to slight variations in the observed locations of transition inception and completion at different cross-stream locations, the uncertainty in these quantities is conservatively estimated to be $\pm 4 \mathrm{~mm}$.

An Analog Devices RTI834-H data acquisition system is used to sample the output of the hot-wire anemometer at $8 \mathrm{kHz}$ over 20 measurement cycles, with each cycle containing 8192 samples. The signal is low-pass filtered at $3.8 \mathrm{kHz}$ prior to A/D conversion. Averaging with different numbers of cycles confirmed the chosen 20 cycles to be more than adequate to achieve statistical convergence for the mean velocity, turbulence, and intermittency measurements. Furthermore, spectral analysis of the 
hot-wire signal sampled at $20 \mathrm{kHz}$ confirmed the sampling rate of $8 \mathrm{kHz}$ and $\mathrm{LP}$ cutoff frequency of $3.8 \mathrm{kHz}$ to be sufficient for resolving the turbulent fluctuations. For the cases with periodic free-stream unsteadiness, the measurement cycles were synchronized with the free-stream oscillations by triggering the data acquisition system once per revolution of the flaps on the unsteady-flow generator.

\subsection{Data Analysis}

A data-reduction program was developed by the author specifically for processing the raw hot-wire signals of the present study. This program computes the mean flow velocity, turbulence intensity and length scale, flow intermittency, and the Fourier and wavelet spectra at each position the hot-wire is traversed. A second program was developed to compute the boundary layer integral parameters at each of the streamwise locations where the boundary layer is traversed. Many of the quantities output by these programs, such as mean velocity, boundary layer integral parameters, and Fourier spectra, are straightforward to compute using standard techniques. The methods used to obtain the less commonly used parameters are described in the following sections. The source code of the data processing software is included on the compact disc provided with the thesis.

\subsubsection{Intermittency Distributions}

Streamwise and cross-stream intermittency distributions in the boundary layer are computed by conditional sampling of the stored hot-wire signals. For the attached flow test cases, the intermittency distributions presented represent the peak values 
from the cross-stream traverses. For separation bubble test cases, the intermittency distributions presented are at a fixed distance from the surface, as described in Section 3.2.2 above.

The algorithm used to computed the intermittency is that described by Volino et al. (2001). This algorithm differentiates between turbulent and non-turbulent portions of the hot-wire trace by evaluating the magnitudes of the first and second derivatives of the velocity signal $\left(d U / d t\right.$ and $\left.d^{2} U / d t^{2}\right)$. A large slope $(d U / d t)$ indicates a rapidly changing velocity (i.e. a turbulent fluctuation), and the second derivative is included in the analysis to avoid "drop-outs" at local maxima and minima. For each sample in the hot-wire trace, these derivatives are computed by central differencing, and compared to threshold values. If either derivative exceeds its threshold, the flow is declared to be instantaneously turbulent. The result is stored in a flow state parameter, $\beta$, which is set to 1 if the flow is deemed turbulent, and 0 otherwise. The intermittency is then obtained as the time-average of this flow state parameter.

Volino et al. (2001) propose a value of the slope threshold that scales on the local free-stream velocity. Their suggested scaling factor is used by default in the current work, although it can easily be adjusted by the user, if necessary. The threshold for the second derivative, initially set to 0 , is adjusted automatically by the algorithm until the time-averages of $\beta$ based solely on $d^{2} U / d t^{2}$ and on $d U / d t$ are equal. These two distributions are then combined, and the resulting $\beta$ distribution is lowpass filtered to further eliminate drop-outs in the signal, using the cutoff frequency suggested by Volino et al. (2001). 
Based on comparison of the hot-wire signals and the output of the algorithm, the threshold scaling factor suggested by Volino et al. (2001) is found to be appropriate for low and moderate values of free-stream turbulence intensity. However, in the test cases with the highest free-stream turbulence levels $(T u>5 \%)$, significant velocity fluctuations caused by buffeting of the boundary layer by the turbulent eddies in the free-stream were observed. An example of such a case is shown in Fig. 3.5. These fluctuations can easily be misinterpreted by the algorithm as turbulence generated within the boundary layer, resulting in inaccurate estimates of the point of transition inception and of the intermittency distribution. Fortunately, the frequency and amplitude of these fluctuations were in most cases distinctly different from those associated with the onset of transition, as shown in Fig. 3.5. Digital high-pass, lowpass, and notch filters were incorporated into the algorithm as options to remove the undesired frequencies from the signal prior to processing, reducing the number of instances where buffeting of the boundary layer was misinterpreted as turbulence activity in the boundary layer. The filtering frequencies were determined by visual inspection of the velocity traces.

In addition to the problems outlined above, there was sometimes a decrease in both the frequency and amplitude of the turbulent velocity fluctuations toward the end of transition, and in the fully turbulent boundary layer. In these cases, the use of a constant scaling factor for the thresholds as suggested by Volino et al. (2001) was found to provide intermittency values in the fully turbulent boundary layer as low as 0.7 . To avoid this problem, at each streamwise location a preliminary cross-stream distribution of intermittency was first obtained with the default setting 


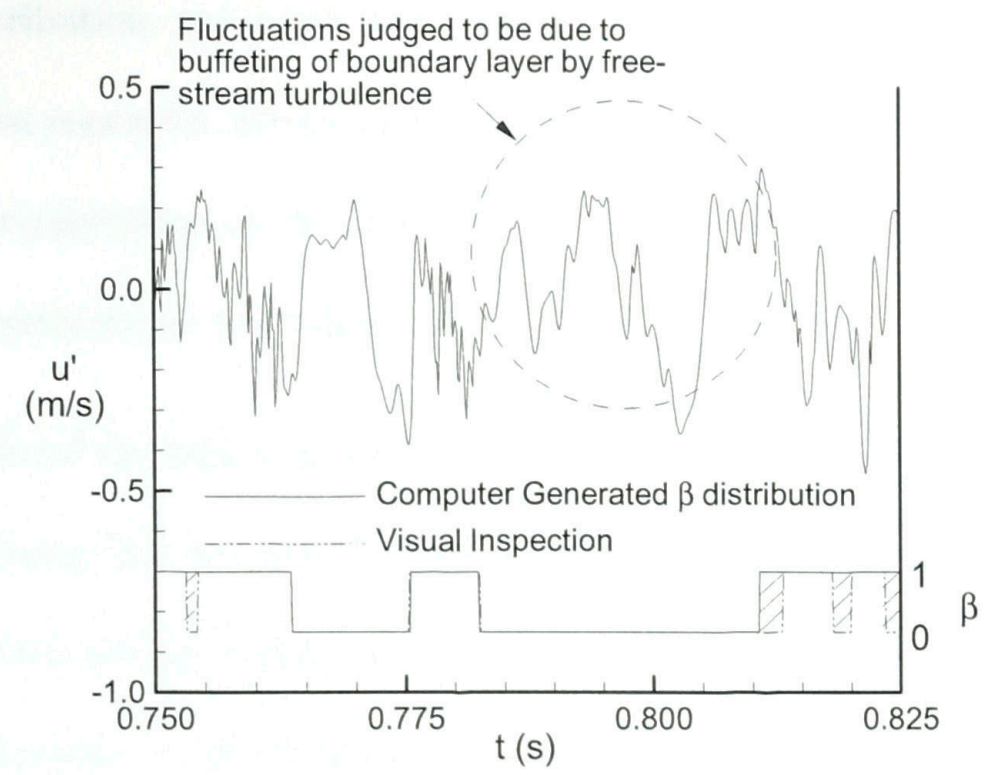

Figure 3.5: Typical hot-wire signal in the boundary layer, and the deduced local flow state

of the velocity slope threshold. Next, the temporal variation of the local flow state parameter, $\beta$ (0: laminar; 1 : turbulent), was determined visually for one of the twenty measurement cycles at the cross-stream location of peak intermittency. The algorithm was then provided with this distribution and searched for the value of the threshold that yielded the closest match to the visually determined distribution. The final values of intermittency at the streamwise location in question were then computed using this optimized threshold value.

The cross-hatched regions in Fig. 3.5 represent the extent of disagreement between the visually determined distribution of the flow state parameter $\beta$, and the computed version. In cases with low or moderate free-stream turbulence, the agreement between visually and computationally established distributions of $\beta$ was better than that displayed in Fig. 3.5. Recognizing that there is uncertainty in the visually established $\beta$ distribution itself, a conservative estimate for the uncertainty 
in the $\beta$ distribution, and hence the computed intermittency, is about \pm 0.05 in the mid-transition region for all test cases. Near the transition inception and completion locations, interpretation of the hot-wire signals is typically much less difficult, and thus the uncertainty in $\gamma$ becomes negligible as the intermittency approaches 0 or 1 .

In spite of the high level of sophistication of the automated data processing, it was still necessary in a few cases to determine the intermittency values solely by visual inspection of the velocity traces. This was particularly true in the fully laminar region, where the algorithm tended to produce occasional non-zero intermittency values under high free-stream turbulence conditions. The algorithm was also found to be unreliable in computing the intermittency for some of the longest transition processes. In these cases, the signature of the free-stream and boundary layer turbulence fluctuations changed significantly from the beginning to the end of the transition process. This is believed to be caused by distortion of turbulent eddies by flowpath contraction as the flow is accelerated, as well as by decay of free-stream turbulence eddies with downstream distance.

For the case with $6.1<T u_{r e f}<6.8 \%$ (Grid \#4), the velocity fluctuations due to buffeting of the boundary layer by the free-stream turbulence were extremely difficult to distinguish from the turbulent fluctuations generated within the boundary layer. For this test case, it was not possible to compute the intermittency distributions using the conditional sampling technique to any level of confidence, even after careful visual inspection of the velocity traces. It was therefore decided to estimate the transition inception point and streamwise variation of intermittency for this test case 
by comparing the experimental shape factor $\left(H=\delta^{*} / \theta\right)$ distribution to the laminar version predicted using the method of Thwaites (1949). An example is shown in Fig. 3.6 for $2.2<T u_{\text {ref }}<2.5 \%$ (Grid \#1), $R e_{L}=350,000$. Due to the influence of the free-stream turbulence, the shape factor in the laminar portion of the boundary layer does not correspond precisely to the prediction. However, the transition inception location was found to correspond reasonably well to the point at which the measured shape factor begins to diverge rapidly from the laminar prediction. Additionally, for the test cases in question, the measured shape factor tends toward a constant value in the fully turbulent region of the boundary layer, which can then be used to indicate transition completion. Between the points of inception and completion of transition, the $\gamma$ distribution is estimated by linear interpolation between the experimental shape factors at the deduced locations of transition inception and completion. In Fig. 3.6, the intermittency computed using this method is seen to be in reasonable agreement with the intermittency based on the time variation of the velocity signal.

The transition inception locations presented in the following chapters are the locations at which turbulent spots were first observed, by visual inspection of the velocity traces. In many of the test cases, the intermittency distribution differs significantly from the "universal" intermittency distribution of Narasimha (1957, 1985). In these cases, establishing transition inception through visual inspection of the velocity traces is deemed more reliable than alternative methods such as extrapolation of the linear portion of the universal intermittency function (Narasimha, 1957, 1985) or using an arbitrary low value of intermittency. 


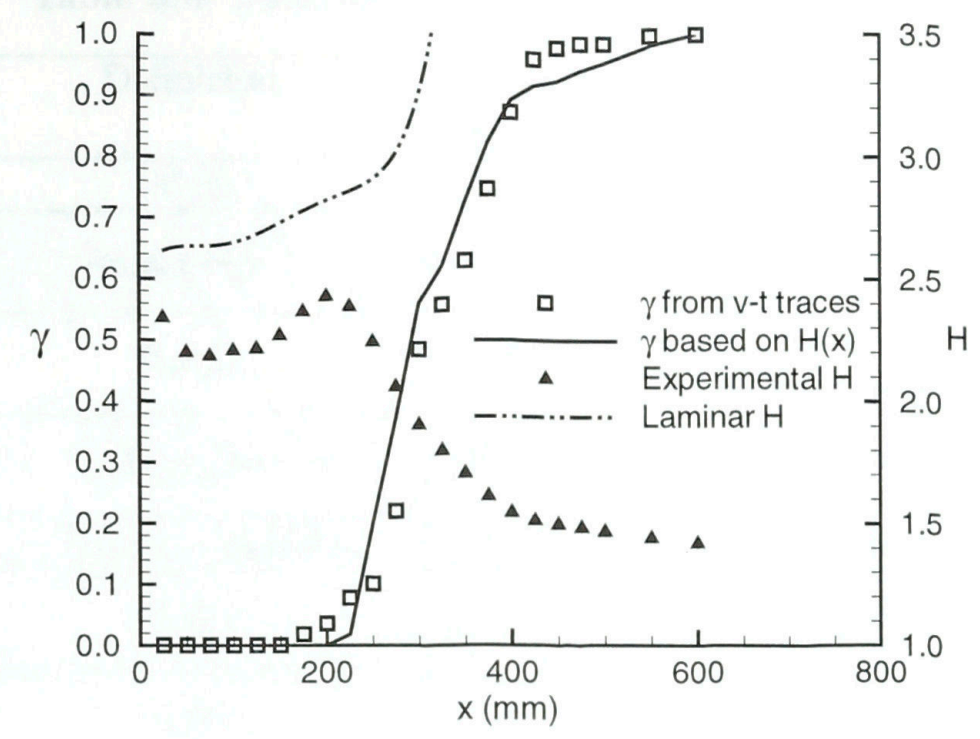

Figure 3.6: Comparison of $\gamma(x)$ values obtained through $v-t$ traces and $H(x)$ distribution ( $C_{P 3}$ pressure distribution; $T u_{r e f}=2.2-2.5 \% ; R e_{L}=350,000$ )

\subsection{Summary of Experimental Uncertainties}

The estimated uncertainties of several measured quantities are quoted in the text of the previous section. However, when developing transition models based on the current experimental data, it is necessary also to estimate the uncertainty in the computed quantities upon which such models are based. For reference, the uncertainties in the measured and computed quantities are summarized in Table 3.3, along with a brief description of the most significant factor contributing to the respective uncertainty values of these quantities, and/or the means by which the uncertainty is estimated. 
Table 3.3: Summary of experimental uncertainties

\begin{tabular}{|c|c|c|c|}
\hline Qty. & Definition & $\begin{array}{l}\text { Estimated } \\
\text { Uncertainty }\end{array}$ & Primary source \\
\hline$H$ & $\delta^{*} / \theta$ & $\pm 0.1^{a}, \pm 0.2^{b}$ & Uncertainty in $\delta^{*}$ and $\theta$ \\
\hline $\log _{10}(N)$ & $\log _{10}\left(\frac{n \sigma \theta^{3}}{\nu}\right)$ & \pm 0.1 & $\begin{array}{l}\text { Combined uncertainty in } \\
\gamma \text { and } \theta\end{array}$ \\
\hline$R e_{L}$ & $U_{e} L / \nu$ & $\pm 5 \%$ & $\begin{array}{l}\text { Level of control of } U_{r e f} \\
\text { during experiments }\end{array}$ \\
\hline$R e_{s}$ & $U_{e} x_{t s} / \nu$ & $\pm 5 \mathrm{~mm}$ & $\begin{array}{l}\text { Extrapolation of dividing } \\
\text { streamline to the surface }\end{array}$ \\
\hline$R e_{s-t s}$ & $U_{e}\left(x_{t s}-x_{s}\right) / \nu$ & $\pm 3 \times 10^{-3}$ & Uncertainty in $x_{s}$ and $x_{t s}$ \\
\hline$R e_{\theta}$ & $U_{e} \theta / \nu$ & \pm 10 & $\begin{array}{l}\text { Combined uncertainties in } \\
U_{e} \text { and } \theta\end{array}$ \\
\hline $\operatorname{Re}_{\delta^{*}}$ & $U_{e} \delta^{*} \nu$ & \pm 20 & $\begin{array}{l}\text { Combined uncertainties in } \\
U_{e} \text { and } \delta^{*}\end{array}$ \\
\hline$S r$ & $f L / U_{e}$ & $\pm 5 \%$ & Uncertainty in $U_{e}$ \\
\hline$S_{s k}$ & $\frac{1}{k_{r m s}^{3} N} \sum_{i=1}^{N}\left(y_{i}-\mu\right)^{3}$ & \pm 0.1 & $\begin{array}{l}\text { Precision of surface scan } \\
\text { measurements }\end{array}$ \\
\hline$T F$ & $T u_{r e f}\left(\frac{L}{\Lambda_{s}}\right)^{1 / 5}$ & $\pm 0.4^{c}$ & $\begin{array}{l}\text { Combined uncertainties in } \\
T u \text { and } \Lambda_{s}\end{array}$ \\
\hline$T u$ & $\sqrt{\frac{1}{N} \sum_{i=1}^{N}\left(U_{i} / U-1\right)^{2}}$ & $\pm 0.2^{c}$ & $\begin{array}{l}\text { Repeatability of } \\
\text { measurement }\end{array}$ \\
\hline$U$ & $\frac{1}{N} \sum_{i=1}^{N} U_{i}$ & $\pm 2 \%^{d}, \pm 4 \%^{e}$ & $\begin{array}{l}\text { Drift of hot-wire calibration } \\
\text { constants }\end{array}$ \\
\hline$W$ & $\frac{2 N \Delta x}{n}$ & $\pm 7 \%$ & $\begin{array}{l}\text { Repeatability of } \\
\text { measurement }\end{array}$ \\
\hline$\delta^{*}$ & $\int_{0}^{\infty}\left(1-\frac{U}{U_{e}}\right) d y$ & $\begin{array}{l} \pm 0.05 \mathrm{~mm}^{a}, \\
\pm 0.15 \mathrm{~mm}^{b}\end{array}$ & $\begin{array}{l}\text { Uncertainty in probe } \\
\text { position at } y=0\end{array}$ \\
\hline$\gamma$ & $\frac{1}{N} \sum_{i-1}^{N} \beta_{i}$ & $\pm 5 \%$ & $\begin{array}{l}\text { Uncertainty in } \beta \\
\text { distribution (note: } \\
\text { uncertainty in } \gamma \text { approaches } \\
0 \text { as } \gamma \rightarrow 1 \text { and } \gamma \rightarrow 0 \text { ) }\end{array}$ \\
\hline$\eta$ & $\frac{\nu}{U_{e}^{2}} \frac{d U_{e}}{d x}$ & $\pm 15 \%$ & $\begin{array}{l}\text { Differentiation of velocity } \\
\text { distribution }\end{array}$ \\
\hline$\lambda_{\theta}$ & $\frac{\theta^{2}}{\nu} \frac{d U_{e}}{d x}$ & $\pm 15 \%$ & $\begin{array}{l}\text { Differentiation of velocity } \\
\text { distribution }\end{array}$ \\
\hline$\Lambda_{s}$ & turbulence length scale & $\pm 30 \%$ & $\begin{array}{l}\text { Based on scatter in data at } \\
\text { different measurement } \\
\text { positions }\end{array}$ \\
\hline$\theta$ & $\int_{0}^{\infty} \frac{U}{U_{e}}\left(1-\frac{U}{U_{e}}\right) d y$ & $\begin{array}{l} \pm 0.02 \mathrm{~mm}^{a} \\
\pm 0.05 \mathrm{~mm}^{b}\end{array}$ & $\begin{array}{l}\text { Uncertainty in probe } \\
\text { position at } y=0\end{array}$ \\
\hline
\end{tabular}

a: Smooth surface; b: Rough surfaces; c: $T F$ and $T u$ expressed in $\%$; d: $U \geq 0.5 \mathrm{~m} / \mathrm{s}$; e: $U<0.5 \mathrm{~m} / \mathrm{s}$ 


\section{Chapter 4}

\section{Effects of Pressure Gradient and Free-Stream Turbulence on Attached Flow Transition}

As described in the Introduction, streamwise variations in pressure gradient within the transition region can result in a significant departure from the universal intermittency distribution described by Narasimha (1957). The most recent model to predict this so-called sub-transition is that of Solomon et al. (1996), which allows for variations in the spot propagation characteristics with pressure gradient. As will be shown in this chapter, the effectiveness of this model is limited by inconsistent accuracy in predicting the turbulent spot production characteristics. In addition, Solomon et al. (1996) acknowledged that their model may need adjustment to account for the effects of free-stream turbulence and Reynolds number. This portion of the study addresses these issues in the context of transition in attached boundary layers. 


\subsection{Test Matrix}

The purpose of this portion of the study is to establish the effects of freestream turbulence and streamwise pressure gradient on the process of attached flow transition. Accordingly, the test matrix is based primarily on systematic variations in the free-stream turbulence level and pressure distribution. To establish sensitivity of these effects to Reynolds number, up to four variations in the flow Reynolds number are considered for each of six free-stream turbulence levels and three pressure distributions. These Reynolds numbers, based on the reference velocity $U_{r e f}$ and plate length $L$, are $930,000,650,000,470,000$, and 350,000 , which were kept constant to within $\pm 5 \%$. The corresponding values of $U_{\text {ref }}$ are about $12.0,8.4,6.0$ and $4.5 \mathrm{~m} / \mathrm{s}$, which were adjusted slightly during the course of the experiments to compensate for flow temperature variations of about $\pm 3^{\circ} \mathrm{C}$.

The surface pressure distributions are shown for three of the test cases in Fig. 4.1. For the $C_{P 1}$ and $C_{P 2}$ pressure distributions, a strong adverse pressure gradient is imposed on the boundary layer by adjusting the ceiling to first converge towards the test plate, and diverge from it further downstream. Both of these pressure distributions resulted in a separation bubble for low and moderate freestream turbulence conditions $\left(T u_{r e f} \leq \sim 5 \%\right)$. The initial accelerations for these two pressure distributions are roughly the same, but a stronger adverse pressure gradient is imposed on the test plate with the $C_{P 1}$ pressure distribution. For the $C_{P 3}$ pressure distribution, a milder adverse pressure gradient is imposed on the boundary layer, with no significant initial acceleration. 


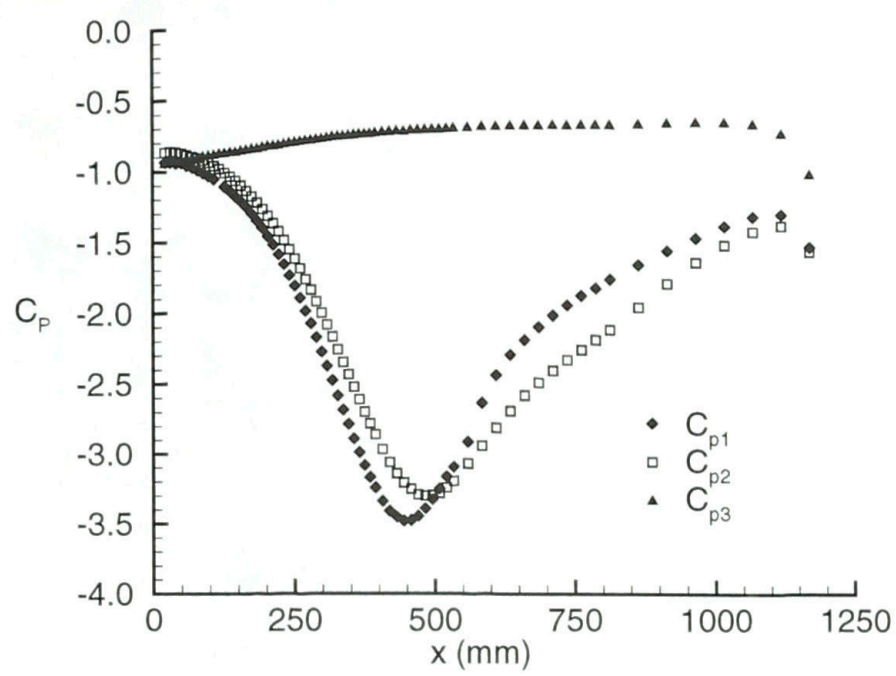

\section{Figure 4.1: Surface static pressure distributions $\left(R e_{L}=\right.$ $350,000)$}

The type of pressure distribution, and the intensity and integral length scale of free-stream turbulence for each of the test cases are summarized in Table 4.1. The parameter $T F$, listed in the last column of this table is Taylor's turbulence factor, $T F=T u\left(\frac{L}{\Lambda_{s}}\right)^{0.2}$ (Eq. 2.12).

For some of the test cases with the $C_{P 1}$ and $C_{P 2}$ pressure distributions, transition occurred in a separation bubble at the lowest flow Reynolds number $\left(R e_{L}=350,000\right)$. These cases were discussed in previous studies by Yaras (2001, 2002), and are therefore not included in Table 4.1.

As noted earlier, the reference free-stream turbulence level is quantified through $y$-direction traverses of the flow $10 \mathrm{~mm}$ upstream of the test-plate leading edge. The sampling rates and sample counts are chosen to be the same as those used in the boundary layer traverses. Since a single-wire sensor, oriented parallel to the test surface, is used for the measurements, the tabulated turbulence quantities 
Table 4.1: Flow Reynolds number, type of pressure distribution, and free-stream turbulence levels

\begin{tabular}{|c|c|c|c|c|c|}
\hline $\begin{array}{c}C_{P} \\
\text { Dist. }\end{array}$ & $\begin{array}{c}R e_{L} \\
\left(\times 10^{3}\right)\end{array}$ & $\begin{array}{c}\text { Grid } \\
\#\end{array}$ & $\begin{array}{c}T u_{\text {ref }} \\
(\%)\end{array}$ & $\begin{array}{c}\Lambda_{s} \\
(\mathrm{~mm})\end{array}$ & $\begin{array}{l}\text { TF } \\
(\%)\end{array}$ \\
\hline 1 & 470 & 2 & 4.4 & 11 & 11.2 \\
\hline 1 & 350 & 4 & 6.4 & 24 & 14.0 \\
\hline 1 & 470 & 4 & 6.8 & 18 & 15.7 \\
\hline 2 & 470 & 4 & 6.8 & 23 & 15.1 \\
\hline 2 & 350 & 5 & 8.9 & 17 & 20.8 \\
\hline 2 & 470 & 5 & 9.0 & 18 & 21.0 \\
\hline 3 & 650 & 0 & 0.7 & 340 & 0.9 \\
\hline 3 & 930 & 0 & 0.5 & 530 & 0.6 \\
\hline 3 & 650 & 1 & 2.5 & 12 & 6.3 \\
\hline 3 & 350 & 1 & 2.3 & 6 & 6.7 \\
\hline 3 & 470 & 1 & 2.2 & 8 & 6.0 \\
\hline 3 & 650 & 3 & 3.8 & 17 & 9.0 \\
\hline 3 & 930 & 3 & 3.8 & 12 & 9.6 \\
\hline 3 & 350 & 3 & 3.7 & 26 & 8.0 \\
\hline 3 & 470 & 3 & 3.9 & 15 & 9.4 \\
\hline 3 & 650 & 2 & 4.6 & 10 & 11.9 \\
\hline 3 & 350 & 2 & 4.3 & 10 & 11.3 \\
\hline 3 & 470 & 2 & 4.4 & 11 & 11.4 \\
\hline 3 & 930 & 4 & 6.5 & 26 & 14.0 \\
\hline 3 & 350 & 4 & 6.2 & 28 & 13.2 \\
\hline 3 & 470 & 4 & 6.1 & 26 & 13.1 \\
\hline
\end{tabular}

are the result of only the streamwise fluctuations in velocity. Nonetheless, based on the geometry of the flowpath, the turbulence is expected to be nearly isotropic, and the measured turbulence intensities are within about $0.5 \%$ of those predicted by a correlation proposed by Frenkiel (1948), with an empirical scaling coefficient taken as suggested by Roach (1997) for square-mesh arrays of square bars. The integral length scales are calculated by autocorrelation of the hot-wire signals, until the first zero-crossing during the integration process. These length scales are generally in reasonable agreement with the empirical correlation proposed by Roach (1997). The 
given turbulence intensities and integral length scales are the average of the values obtained at 49 evenly spaced points over $120 \mathrm{~mm}$ in height, starting $5 \mathrm{~mm}$ above the test plate. Based on these measurements, the turbulence and mean velocity fields are deemed to be reasonably homogeneous. The exceptions are the cases using perforated plate \#4 (Table 3.2) as the free-stream turbulence generator. The turbulence intensity and length scale values quoted for these cases were calculated as the average of the measurements over the first $60 \mathrm{~mm}$ length of the traverse, within which the spatial uniformity of turbulence is comparable to those of the remaining test cases.

The pressure distributions $C_{P 1}, C_{P 2}$, and $C_{P 3}$ in Table 4.1 were obtained by adjusting the ceiling geometry of the test section. The variations in the flow Reynolds number and free-stream turbulence properties may cause changes in the growth rate of the boundary layers on the flexible ceiling and test-plate surfaces. For the same geometric setting, this would affect the effective free-stream flow area and its streamwise variation in the test section, hence yield variations in the pressure distribution imposed upon the test-plate boundary layer upstream of the transition region. The extent of these variations are found to be small, as is evident from the trends up to about $x=500 \mathrm{~mm}$ in Figs. 4.2-a and $-\mathrm{b}$. Since the streamwise pressure gradient is the more relevant quantity in relation to the development of a boundary layer than the pressure value itself, an acceleration parameter, $\eta$, rather than the conventional pressure coefficient, $C_{P}$, is presented in these figures. This acceleration parameter is defined as: 

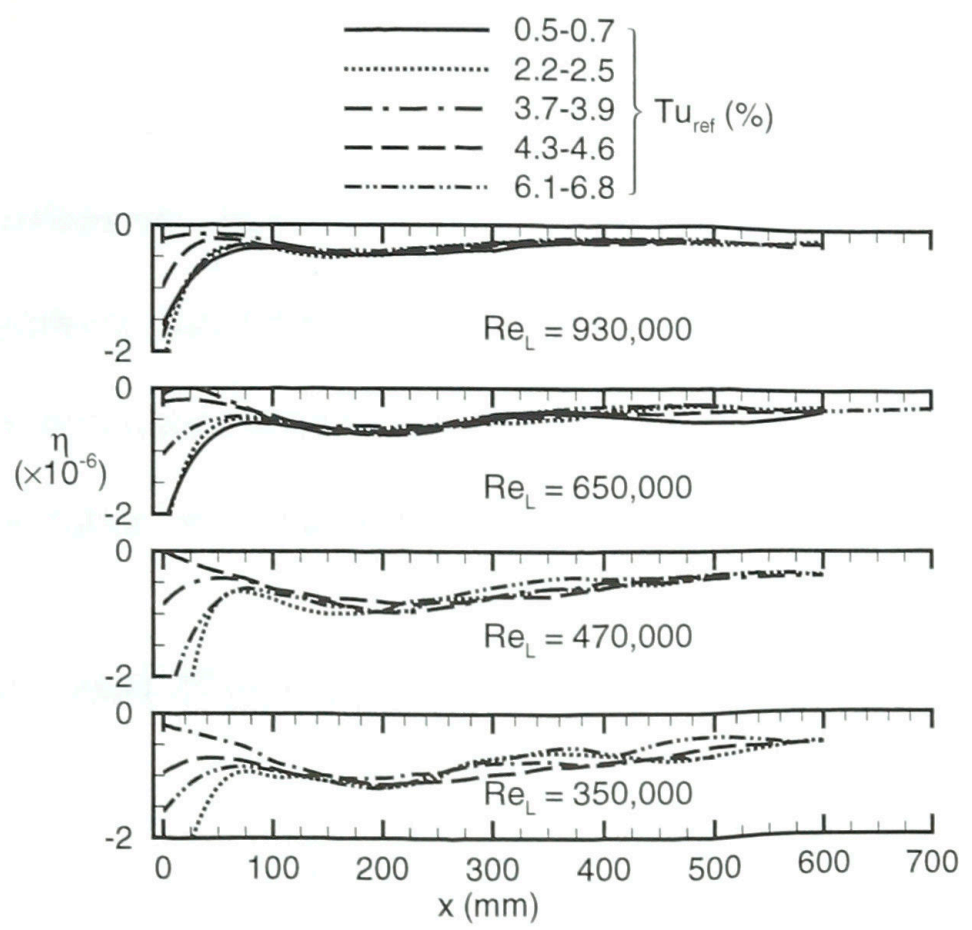

(a) $C_{P 3}$ pressure distribution

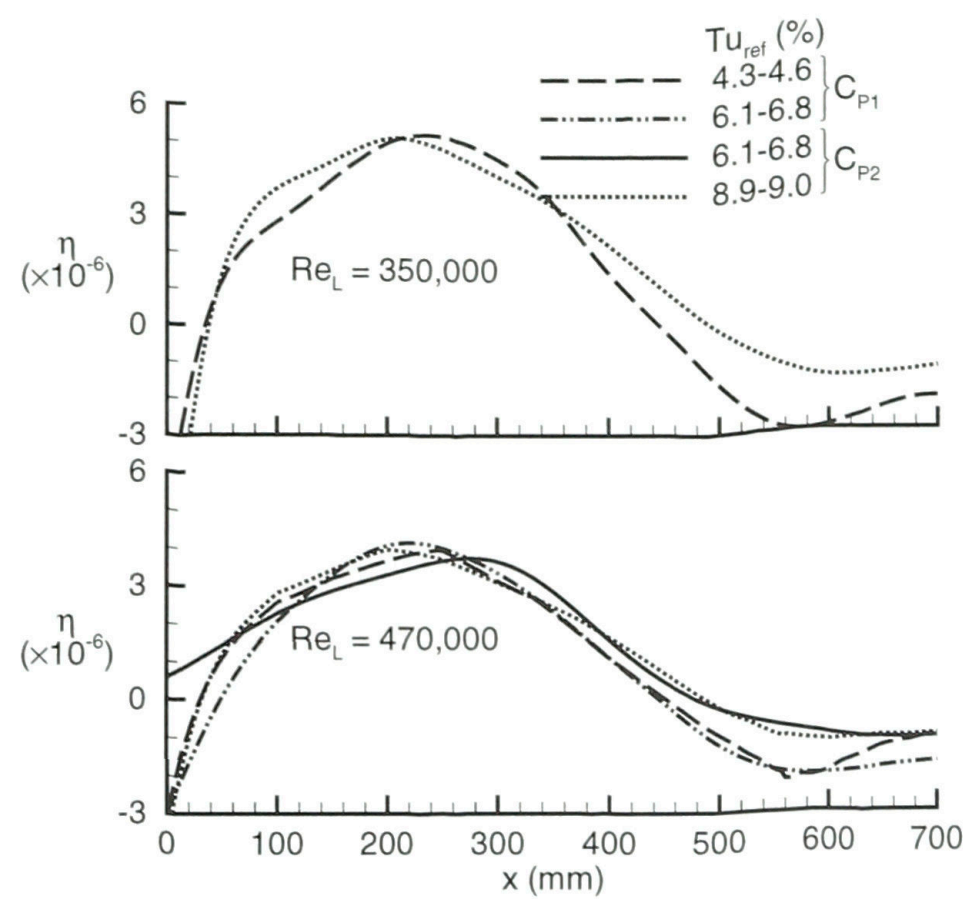

(b) $C_{P 1} \& C_{P 2}$ pressure distributions

Figure 4.2: Streamwise variation of the acceleration parameter 


$$
\eta=\frac{\nu}{U_{e}^{2}} \frac{d U_{e}}{d x}
$$

These $\eta$ distributions are obtained through least-square polynomial curve fits to the surface static pressure distributions. The curve fits are typically performed in three or four partially overlapping segments between $x=0 \mathrm{~mm}$ and $x=1000 \mathrm{~mm}$ to ensure accurate representation of the measured pressure distributions.

\subsection{Results and Discussion}

The location of transition inception, and a number of flow parameters at the point of transition which are known to have an influence on the development of the boundary layer are summarized in Table 4.2. The test cases corresponding to the $C_{P 1}$ distribution are listed first, followed by the $C_{P 2}$ distribution and the $C_{P 3}$ distribution. For each pressure distribution, the test cases are presented in the order of increasing turbulence intensity, and for each turbulence intensity, the cases are presented in the order of increasing flow Reynolds number.

The additional turbulence intensities listed in Table 4.2 are $T u_{t s}$, the local value at the transition inception point, and $\overline{T u}$, the average of the turbulence intensity at transition inception $\left(T u_{t s}\right)$ and just upstream of the leading edge of the test plate $\left(T u_{\text {ref }}\right)$ listed in Table 4.1. This average turbulence intensity is required in the comparison of the present results to the model of Abu-Ghannam \& Shaw (1980). It is worthwhile noting that in cases where transition inception occurred in a region of accelerated flow $\left(\eta_{t s}>0\right)$, the local turbulence intensity at transition inception is 
Table 4.2: Streamwise position and flow parameters at transition inception

\begin{tabular}{|c|c|c|c|c|c|c|c|}
\hline $\begin{array}{c}C_{P} \\
\text { Dist. }\end{array}$ & $\begin{array}{c}R e_{L} \\
\left(\times 10^{3}\right)\end{array}$ & $\begin{array}{c}T u_{\text {ref }} \\
(\%)\end{array}$ & $\begin{array}{c}T u_{t s} \\
(\%)\end{array}$ & $\begin{array}{c}\overline{T u} \\
(\%)\end{array}$ & $\begin{array}{c}x_{t s} \\
(\mathrm{~mm})\end{array}$ & $R e_{\theta_{t s}}$ & $\begin{array}{c}\lambda_{\theta_{t s}} \times 10^{-2} \\
(\mathrm{Eq.} \mathrm{2.7)}\end{array}$ \\
\hline 1 & 470 & 4.4 & 1.7 & 3.1 & 300 & 191 & 11.8 \\
\hline 1 & 350 & 6.4 & 5.0 & 5.7 & 350 & 167 & 9.5 \\
\hline 1 & 470 & 6.8 & 6.0 & 6.4 & 150 & 199 & 12.7 \\
\hline 2 & 470 & 6.8 & 4.1 & 5.5 & 250 & 171 & 11.1 \\
\hline 2 & 350 & 8.9 & 4.1 & 6.5 & 350 & 177 & 10.1 \\
\hline 2 & 470 & 9.0 & 5.9 & 7.5 & 200 & 185 & 13.7 \\
\hline 3 & 650 & 0.7 & 0.7 & 0.7 & 450 & 364 & -6.6 \\
\hline 3 & 930 & 0.5 & 0.5 & 0.5 & 275 & 351 & -5.1 \\
\hline 3 & 350 & 2.3 & 1.9 & 2.1 & 325 & 227 & -3.4 \\
\hline 3 & 470 & 2.2 & 1.9 & 2.1 & 250 & 239 & -4.3 \\
\hline 3 & 650 & 2.5 & 2.0 & 2.3 & 175 & 216 & -3.2 \\
\hline 3 & 350 & 3.7 & 3.3 & 3.5 & 275 & 226 & -4.5 \\
\hline 3 & 470 & 3.9 & 3.3 & 3.6 & 225 & 258 & -5.6 \\
\hline 3 & 650 & 3.8 & 3.4 & 3.6 & 150 & 226 & -3.2 \\
\hline 3 & 930 & 3.8 & 3.3 & 3.6 & 75 & 175 & -0.6 \\
\hline 3 & 350 & 4.3 & 3.5 & 3.9 & 175 & 176 & -3.4 \\
\hline 3 & 470 & 4.4 & 3.7 & 4.1 & 100 & 141 & -1.2 \\
\hline 3 & 650 & 4.6 & 4.0 & 4.3 & 75 & 139 & -0.6 \\
\hline 3 & 350 & 6.2 & 6.2 & 6.2 & 100 & 122 & -1.4 \\
\hline 3 & 470 & 6.1 & 6.1 & 6.1 & 50 & 98 & -0.8 \\
\hline 3 & 930 & 6.5 & 6.4 & 6.5 & 50 & 168 & -1.3 \\
\hline
\end{tabular}

seen to be considerably lower than the reference value at the leading edge of the test plate.

\subsubsection{Transition Inception}

\subsubsection{Effects of Reynolds Number and Free-Stream Turbulence}

For each of the pressure distributions and free-stream turbulence levels considered, the extent of upstream movement of the transition inception point with increasing Reynolds number is shown in Fig. 4.3. As seen in the lower half of this 


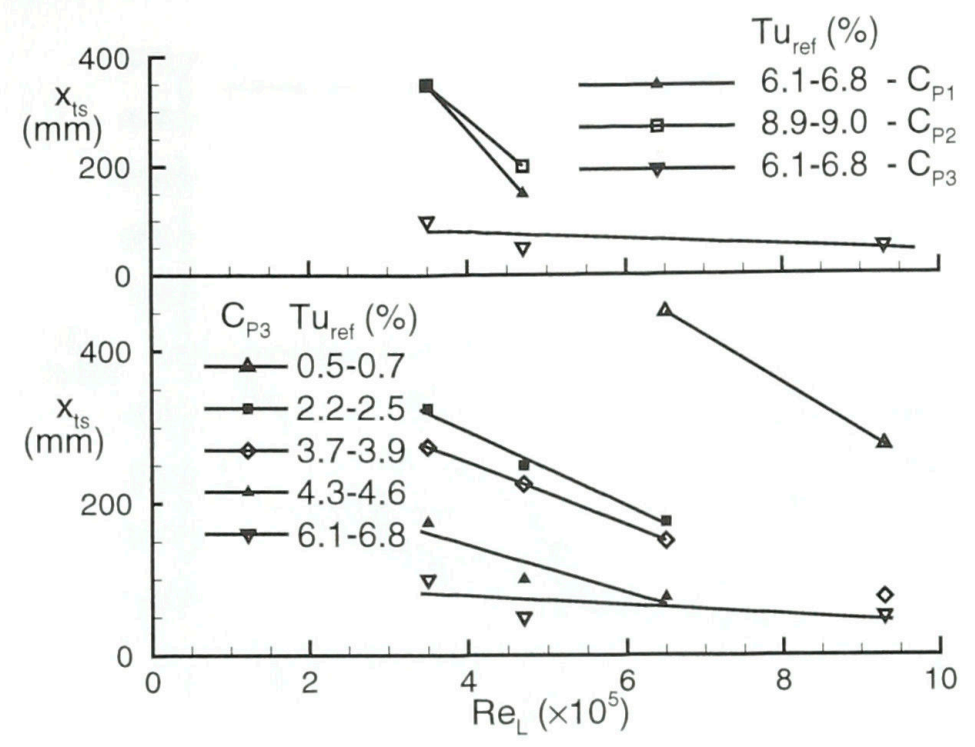

\section{Figure 4.3: Upstream movement of the transition inception location with increasing flow Reynolds number}

figure, the effect of $R e_{L}$ on the transition inception location is reduced significantly as free-stream turbulence is increased. For the highest turbulence intensity, $x_{t s}$ is noted to remain nearly constant for most of the range of flow Reynolds number. The test cases plotted in the upper half of the figure correspond to $\overline{T u}$ values ranging from $5.7 \%$ to $7.5 \%$. The location of transition inception is seen to be notably more sensitive to Reynolds number variations when the free-stream flow over the flat plate is initially accelerated ( $C_{P 1}$ and $C_{P 2}$ pressure distributions). This may be partly due to the fact that the free-stream turbulence intensity decreases more rapidly with distance from the leading edge in these instances.

To better illustrate the effect of free-stream turbulence on transition inception, the data is re-plotted in Fig. 4.4, with $\overline{T u}$ as the $\mathrm{x}$-axis variable. As $\overline{T u}$ is increased, the transition inception point moves upstream, as expected. This movement does not occur at a constant rate however, as is evident from a sudden shift in $x_{t s}$ as $\overline{T u}$ is 


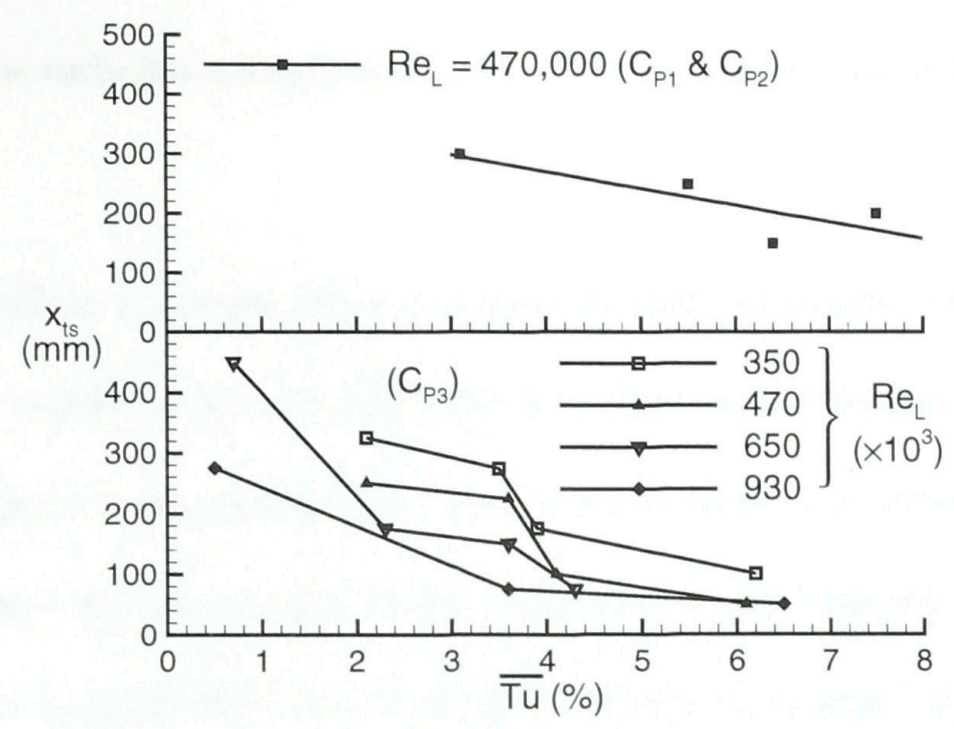

Figure 4.4: Upstream movement of the transition inception location with increasing turbulence intensity

increased from about $3.5 \%$ to $4 \%$, particularly at the lower flow Reynolds numbers. As listed in Table 3.2, the turbulence grids for the two test cases (\#2 and \#3) were of different open area ratios. This was done in an attempt to have some independent control of the intensity and length scale of free-stream turbulence. From the values of $T u_{r e f}$ and $\Lambda_{s}$ for these turbulence grids in Table 4.1 (Grid \#2: $3.9<\overline{T u}<4.3 \%$ and $10<\Lambda_{s}<12 \mathrm{~mm}$; Grid \#3: $3.5<\overline{T u}<3.6 \%$ and $\left.12<\Lambda_{s}<26 \mathrm{~mm}\right)$ it can be seen that the different open area ratios provided this control to some extent. The trend with $\overline{T u}$ in Fig. 4.4 for these two grids suggests that there a significant effect of the turbulence length scale on the location of transition inception.

Also of interest are the $C_{P 3}$ test cases at $T u_{\text {ref }}=2.2-2.5 \%$ and at $T u_{\text {ref }}=3.7-3.9 \%$ (Grids \#1 and \#3). As seen in Table 4.2, the locations of transition inception are very close for the two cases at Reynolds numbers $R e_{L}=350,000$, and $R e_{L}=470,000$. The similarity in these transition inception 
locations despite different free-stream turbulence intensities is believed to be due to differences in the turbulence length scale $\left(\Lambda_{s}=6-12 \mathrm{~mm}\right.$ for Grid \#1 vs. $12-26 \mathrm{~mm}$ for Grid \#3).

The trends in $x_{t s}$ noted above demonstrate that the smaller turbulence length scales promote earlier transition. Since the boundary layer thickness at the point of transition inception is approximately $7 \mathrm{~mm}$ in these cases, it is concluded that freestream turbulence with an integral length scale closer to the boundary layer thickness is more effective in promoting transition than turbulence of larger scales. This lends support for the use of a turbulence factor (e.g. Eq. 2.12) rather than the turbulence intensity in developing correlations for the location of transition inception.

\subsubsection{Comparison to Existing Models}

In Figures 4.5 and 4.6, the measured momentum thickness Reynolds numbers at transition onset are compared to the correlations of Abu-Ghannam \& Shaw (1980), Mayle (1991), and Hourmouziadis (1989), which were introduced in Section 2.4 .1 and are repeated below:

Abu-Ghannam \& Shaw (1980):

$$
R e_{\theta_{t s}}=163+e^{F\left(\lambda_{\theta}\right)\left(1-\frac{\overline{T u} \%}{6.91}\right)}
$$

where: 


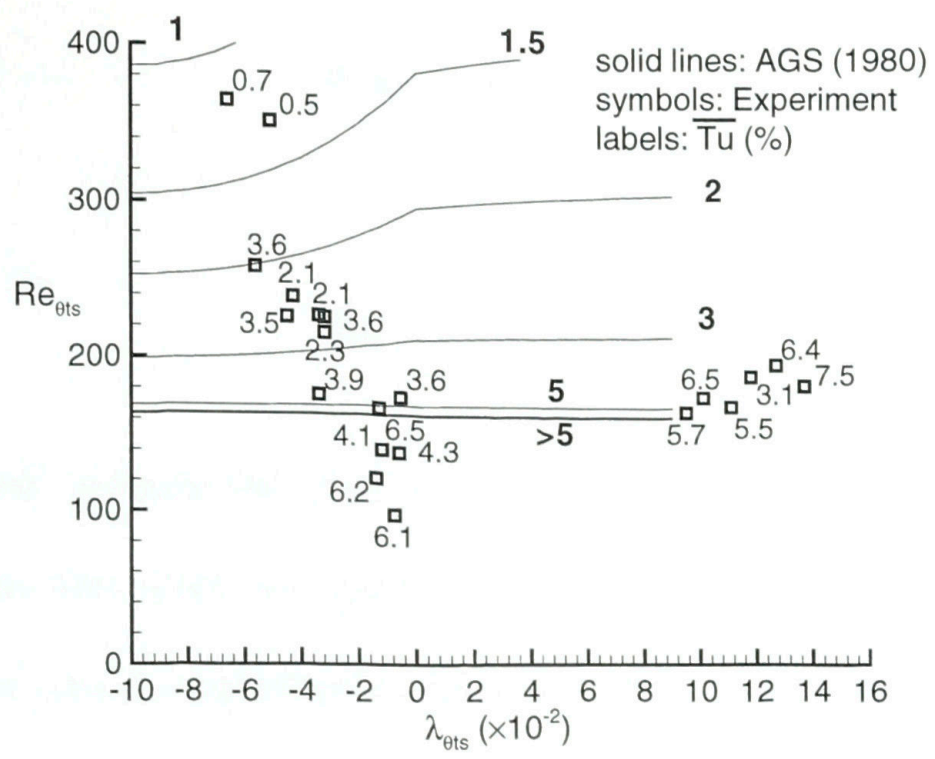

Figure 4.5: Comparison of experimental values of $R e_{\theta_{t s}}$ to the correlation of Abu-Ghannam \& Shaw (1980)

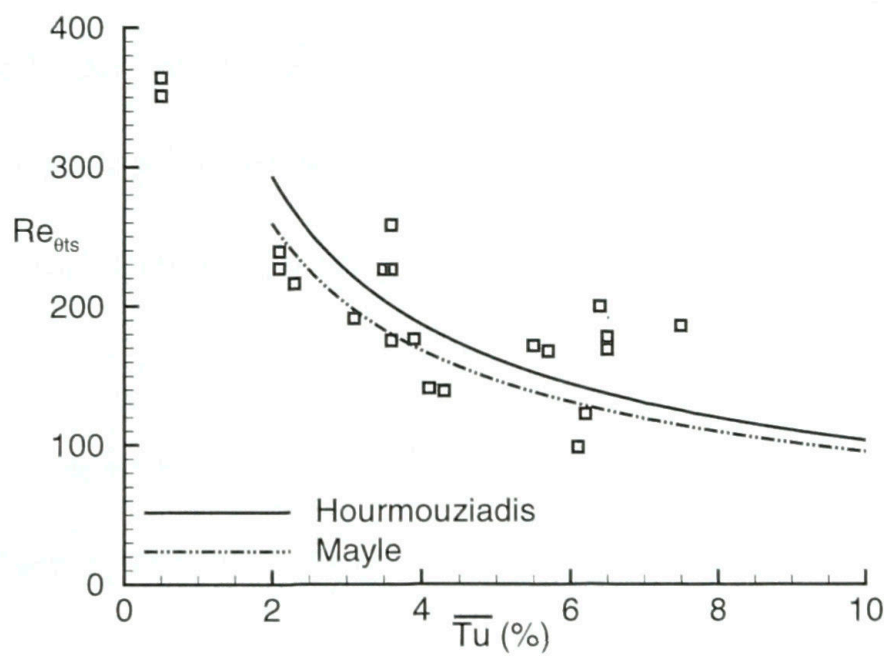

Figure 4.6: Comparison of $R e_{\theta_{t s}}$ to the correlations of Hourmouziadis (1989) and Mayle (1991)

$$
\begin{array}{cc}
F\left(\lambda_{\theta}\right)=6.91+12.75 \lambda_{\theta}+63.64 \lambda_{\theta}{ }^{2} & \left\{\lambda_{\theta} \leq 0\right\} \\
F\left(\lambda_{\theta}\right)=6.91+2.48 \lambda_{\theta}+12.27 \lambda_{\theta}{ }^{2} & \left\{\lambda_{\theta}>0\right\}
\end{array}
$$


Hourmouziadis (1989):

$$
R e_{\theta_{t s}}=460 \overline{T u}^{-0.65}
$$

Mayle (1991):

$$
\operatorname{Re}_{\theta_{t s}}=400 \overline{T u}^{-0.625}
$$

Of these models, perhaps the most widely used is that of Abu-Ghannam \& Shaw (1980). Unlike the other two models, it incorporates the effects of free-stream turbulence and pressure distribution, for both adverse and favourable gradients, on the transition inception point.

As was noted in Section 2.4.1 however, the correlation of Abu-Ghannam \& Shaw (1980) has been artificially forced to correspond to the stability limit $R e_{\theta_{t s}}=$ 163 for flows with high turbulence intensity. While Hourmouziadis' and Mayle's correlations are not similarly constrained, they do not account for effects of pressure gradient. For free-stream turbulence intensities typical of turbomachines, Mayle noted that transition inception in an attached boundary layer is not strongly affected by the pressure distribution. In keeping with this, Mayle suggests that Eq. 4.6 should only be used when the turbulence intensity exceeds $3 \%$. Such a restriction should also be imposed on the model of Hourmouziadis (Eq. 4.5).

In Fig. 4.5, the measured values of $R e_{\theta_{t s}}$ differ somewhat from those predicted by the correlation of Abu-Ghannam \& Shaw (1980) for many of the test cases, particularly under adverse pressure gradient conditions. As was noted in the preceding section, the transition inception locations were determined by visual inspection of 
the velocity traces. However, the data used by Abu-Ghannam \& Shaw (1980) were not based on intermittency measurements. Instead, transition inception was detected through velocity measurements at a fixed location near the surface, as the wind tunnel Reynolds number was gradually increased. A sharp increase in the rate at which this velocity increases with $R e$ provides an indication of the transition inception point passing through the measurement location. Fraser et al. (1994) and Dey \& Narasimha (1988) suggested that this measurement technique results in over-prediction of $R e_{\theta_{t s}}$ by up to $15 \%$. In addition to the different experimental methods, there is some uncertainty in the exact location of transition onset in the present measurements, and consequently in the value of $\lambda_{\theta_{t s}}$, primarily due to the streamwise resolution of the boundary layer traverses. This uncertainty in $\lambda_{\theta_{t s}}$ varies from approximately $15 \%$ for test cases in which $\lambda_{\theta_{t s}}>0$, to $30 \%$ for cases in which $\lambda_{\theta_{t s}}<0$. The increased uncertainty for adverse pressure gradients is due to the more rapid growth in $\theta$ after transition inception in these cases. When comparing the experimental values to the correlation of Abu-Ghannam \& Shaw (1980), the uncertainty in the experimental values of $R e_{\theta_{t s}}$ is judged to be \pm 40 for $\lambda_{\theta_{t s}}<0$, and \pm 20 for $\lambda_{\theta_{t s}}>0$, considering both the streamwise spacing of the boundary layer traverses, and the different methods by which transition inception was determined. This uncertainty is generally larger than the difference between the measured and predicted values in Fig. 4.5, and thus the transition inception locations predicted by the correlation of Abu-Ghannam \& Shaw (1980) are deemed to be supported by the present experiments. The exceptions are the test cases with the $C_{P 3}$ pressure distribution and Grid \#4 $\left(6.1 \%<T u_{r e f}<6.8 \%\right)$ at $R e_{L}=350,000$ and 470,000, and those for Grid \#2 $\left(4.3 \%<T u_{r e f}<4.6 \%\right)$ at 
$R e_{L}=650,000$ and 470,000. In these instances, transition inception occurred below the stability limit of $R e_{\theta_{s}}=163$, indicating that bypass transition occurred in these test cases.

In Fig. 4.6, most experimental data points with free-stream turbulence levels greater than $2 \%$ are seen to be in reasonable agreement with the predictions based on the models of Mayle (1991) and Hourmouziadis (1989). This observation supports the argument of a relatively weak influence of pressure gradient on transition onset for higher free-stream turbulence intensities.

\subsubsection{Streamwise Intermittency Distributions}

\subsubsection{Effect of Pressure Distribution on Transition Rate}

Streamwise distributions of the pressure gradient parameter $\lambda_{\theta}$ are compared in Fig. 4.7 for one test case with the $C_{P 1}$ pressure distribution, and one with the $C_{P 3}$ pressure distribution. The intermittency distributions for these test cases are shown in Fig. 4.8. The cases correspond to the same reference turbulence intensity (Grid \#4, $6.1 \%<T u<6.8 \%)$ and flow Reynolds number $\left(R e_{L}=470,000\right)$. However, due to the different pressure distributions, the transition inception locations are not the same. The noted cases were chosen such that the momentum thickness Reynolds numbers at transition inception are comparable, with the values of $\operatorname{Re}_{\theta_{t s}}$ being 191 and 141 for the $C_{P 1}$ and $C_{P 3}$ cases, respectively. Thus, the differences in the intermittency distributions seen in Fig. 4.8 are due mainly to the different pressure distributions imposed on the boundary layer. The trends in intermittency shown in Fig. 4.8 suggest 
a notable effect of $\lambda_{\theta}$ on the rate of transition. A much greater variation in transition rate is noted for the $C_{P 1}$ test case, for which there is also a much greater variation in the pressure gradient parameter $\lambda_{\theta}$. In particular, the transition process is seen to be accelerated with decreasing values of $\lambda_{\theta}$, as has also been noted by many other researchers (e.g. Chen \& Thyson, 1971; Solomon et al., 1996; Gostelow et al., 1994; Mayle, 1991; Narasimha, 1985; Gostelow et al., 1996).

\subsubsection{Effect of Turbulence Intensity on Transition Rate}

The effect of turbulence intensity on the rate of transition is shown for two flow Reynolds numbers in Figs. 4.9-a \&-b, respectively. Since the goal is to isolate effects of turbulence intensity on transition rate, it is most convenient to consider test cases where variations in free-stream turbulence levels are not accompanied by significant changes in the $\lambda_{\theta}$ distribution in the transition region. The test cases with the $C_{P 3}$ pressure distribution are consistent with this criterion. Among these cases, those that correspond to free-stream turbulence intensity ranges of $2.2 \%<T u_{r e f}<2.5 \%$ (Grid \#1) and $3.7 \%<T u_{r e f}<3.9 \%($ Grid \#3) are directly comparable, since transition inception occurred within one spacing of the boundary layer traverses $(25 \mathrm{~mm})$ for these two turbulence levels. The trends shown in Fig. 4.9 do not indicate a consistent change in the intermittency distribution with free-stream turbulence, with the observed differences being close to the estimated uncertainty in $\gamma$. 


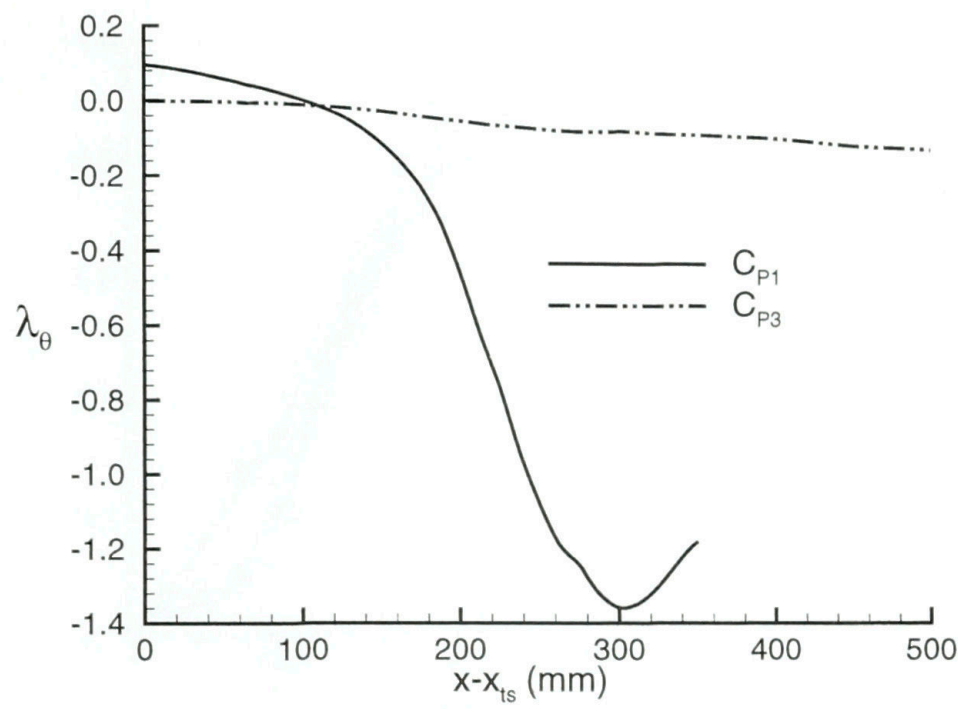

Figure 4.7: Streamwise variation of the pressure gradient parameter $\lambda_{\theta}$ within the transition region $\left(R e_{L}=470,000 ; T u_{r e f}=6.1-6.8 \%\right)$

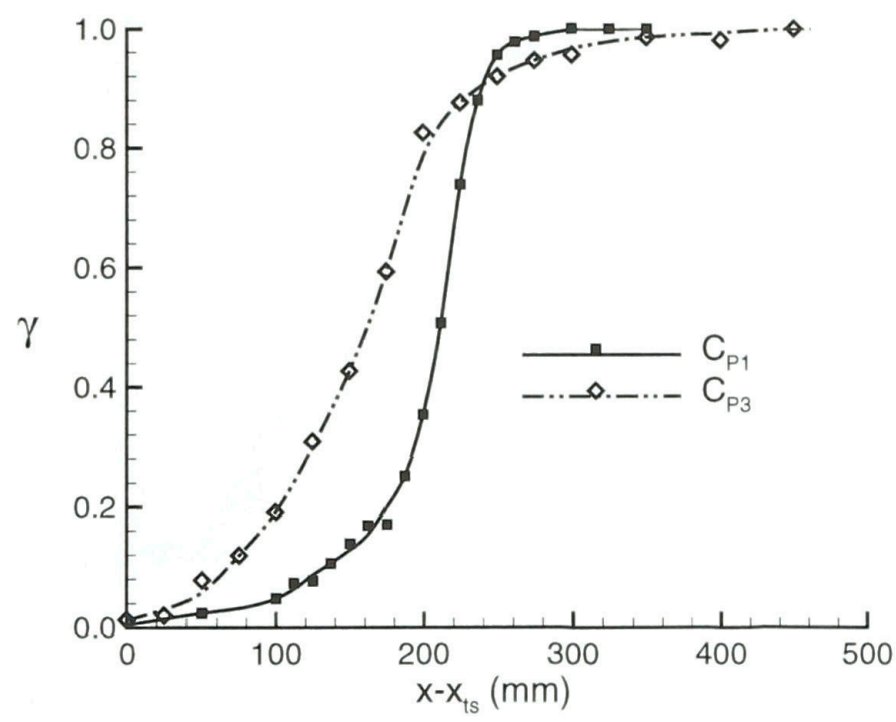

Figure 4.8: Sensitivity of the intermittency distribution to $\lambda_{\theta}\left(R e_{L}=470,000 ; T u_{r e f}=6.1-6.8 \%\right)$ 


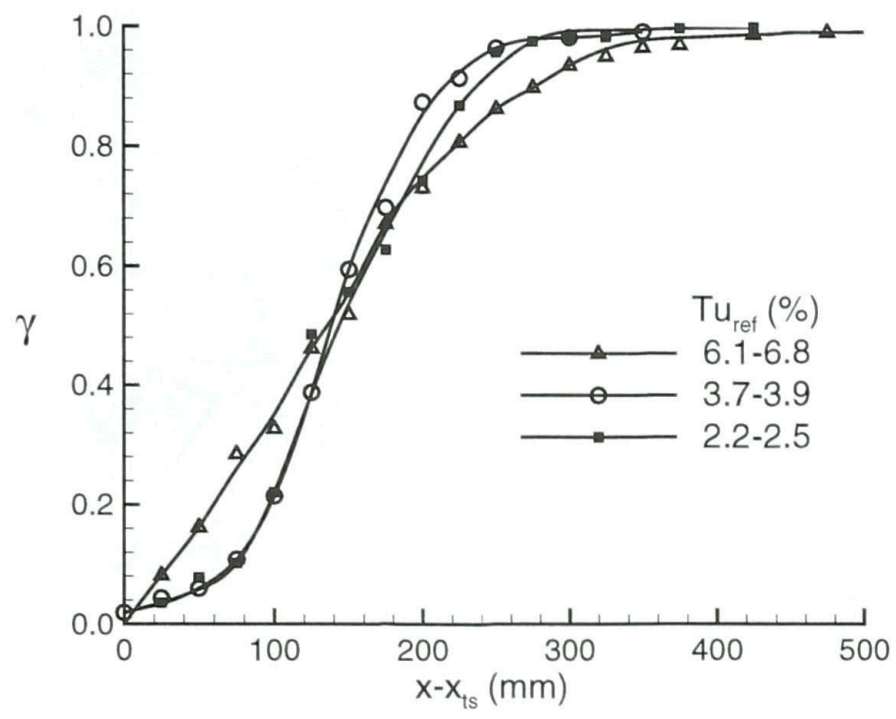

(a) $R e_{L}=650,000$

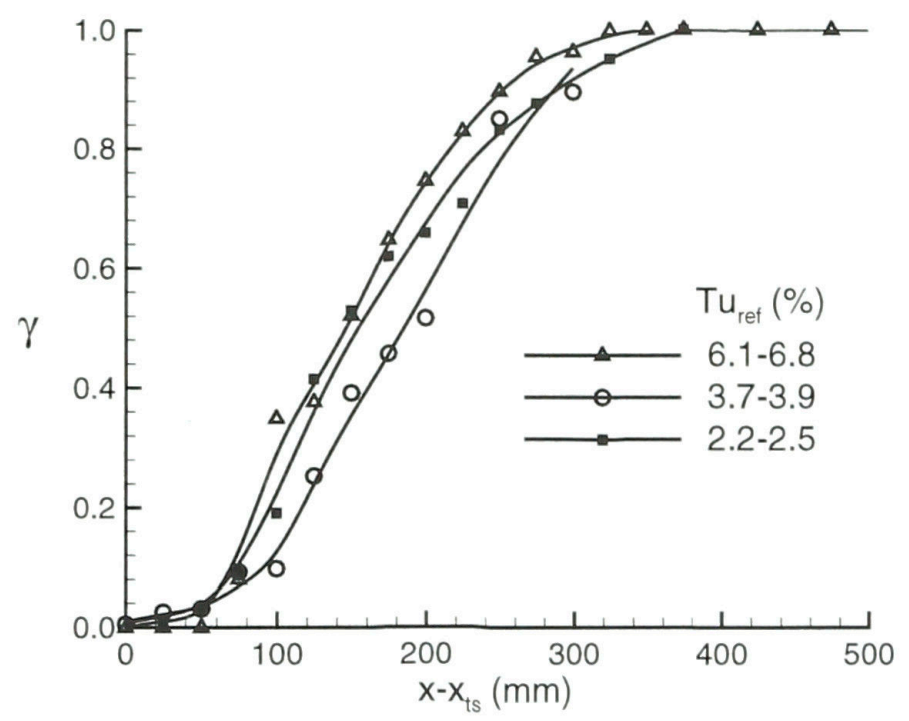

(b) $R e_{L}=470,000$

Figure 4.9: Effect of turbulence intensity on the rate of transition $\left(C_{P 3}\right.$ pressure distribution) 


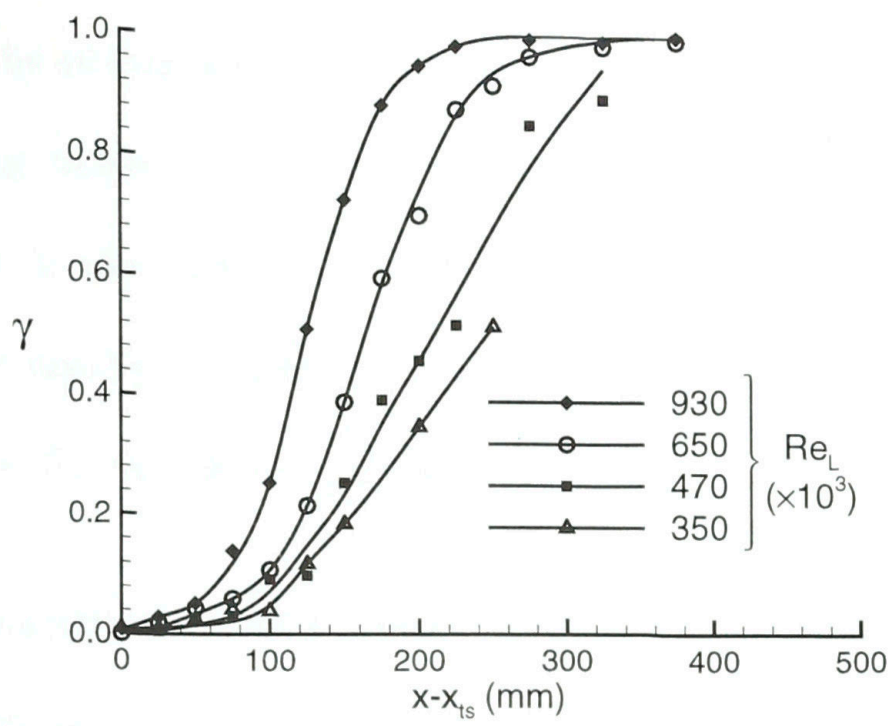

Figure 4.10: Effect of flow Reynolds number on the rate of transition ( $C_{P 3}$ pressure distribution; $T u_{\text {ref }}=3.7-3.9 \%$ )

\subsubsection{Effect of Reynolds Number on Transition Rate}

In Fig. 4.10, the intermittency distributions for the $C_{P 3}$ test cases using turbulence grid \#3 $\left(3.7 \%<T u_{\text {ref }}<3.9 \%\right)$ are compared over a range of flow Reynolds numbers. As with the study of the effect of turbulence intensity presented in the previous section, the comparison is based on the $C_{P 3}$ pressure distribution, to allow for evaluation of Reynolds number effects in the absence of significant variations in $\lambda_{\theta}$. The trends in this figure indicate an increase in the rate of transition with increasing flow Reynolds number. A similar trend was also observed among the test cases using turbulence grid \#1 $\left(2.2 \%<T u_{\text {ref }}<2.5 \%\right)$. However, the effect of Reynolds number on the transition rate was considerably less pronounced for the highest turbulence intensity cases, with turbulence grids \#2 $\left(4.3 \%<T u_{r e f}<4.6 \%\right)$ and \#4 $\left(6.1 \%<T u_{\text {ref }}<6.8 \%\right)$. 
In summary, the most significant factor influencing the rate of transition appears to be the local pressure gradient parameter, with the transition rate increasing with decreasing values of $\lambda_{\theta}$ within the transition zone. To a lesser extent, the transition rate is also influenced by the flow Reynolds number, with transition occurring more rapidly at higher Reynolds numbers, although this effect is not seen to be significant for turbulence intensities above $4 \%$.

\subsubsection{Comparison to Transition Length Model of Solomon, Walker \& Gostelow}

In Fig. 4.11, the intermittency distributions are compared, for selected cases, to the transition length model of Solomon, Walker, \& Gostelow (1996), which was introduced previously in Section 2.4.2, and repeated here for convenience:

$$
\gamma(x)=1-e^{-n \int_{x_{t s}}^{x} \frac{\sigma}{\tan (\alpha) U} d x \int_{x t s}^{x} \tan (\alpha) d x}
$$

$$
\begin{aligned}
& \alpha=4+\frac{22.14}{0.79+2.72 e^{47.63 \lambda_{\theta}}} \\
& \sigma=0.024+\frac{0.604}{1+5 e^{66 \lambda_{\theta}}}
\end{aligned}
$$

$$
\begin{array}{lll}
N=8.6 \times 10^{-4} e^{2.134 \lambda_{\theta_{t s}} \ln \left(T u_{t s}\right)-59.23 \lambda_{\theta_{t s}}-0.564 \ln \left(T u_{t s}\right)} & \left\{\lambda_{\theta t s} \leq 0\right\} & (4.10-\mathrm{a}) \\
N=8.6 \times 10^{-4} e^{-0.564 \ln \left(T u_{t s}\right)-10 \sqrt{\lambda_{\theta_{t s}}}} & \left\{\lambda_{\theta t s}>0\right\} & (4.10-\mathrm{b})
\end{array}
$$




$$
N=\frac{n \sigma \theta_{t s}^{3}}{\nu}
$$

For the $C_{P 3}$ pressure distribution, where transition inception occurred in a region of adverse pressure gradient, the model of Solomon et al. (1996) was found to predict a significantly faster transition rate than was observed experimentally (Fig. 4.11-a). For the $C_{P 1}$ and $C_{P 2}$ pressure distributions, transition inception occurred in a region of favourable pressure gradient, which was followed by a region of adverse pressure gradient. As shown in Fig. 4.11-b, the transition rate predicted by the model of Solomon et al. (1996) (SWG) is substantially more accurate for these test cases, particularly in the adverse pressure gradient region.

The discrepancies between the experimental results and the predictions for the test cases with the $C_{P 3}$ pressure distribution (Fig. 4.11-a), are believed to be the result of inaccurate prediction of the turbulent spot formation rate on the basis of Eq. 4.10a. Gostelow et al. (1994) arrived at this correlation by applying Narasimha's (1957) universal intermittency distribution to measured transition lengths. As is evident in Fig. 13 of the paper by Solomon et al. (1996), the scatter in the normalized spot formation rate when plotted against the variables $\lambda_{\theta}$ and $T u_{t s}$ is substantial. This results in many of the measured spot formation rates differing from the predictions of Eq. 4.10-a by more than 50 percent. An improved model for predicting the spot inception rate will be presented in Chapter 8, which will also be applicable to separation bubbles. 


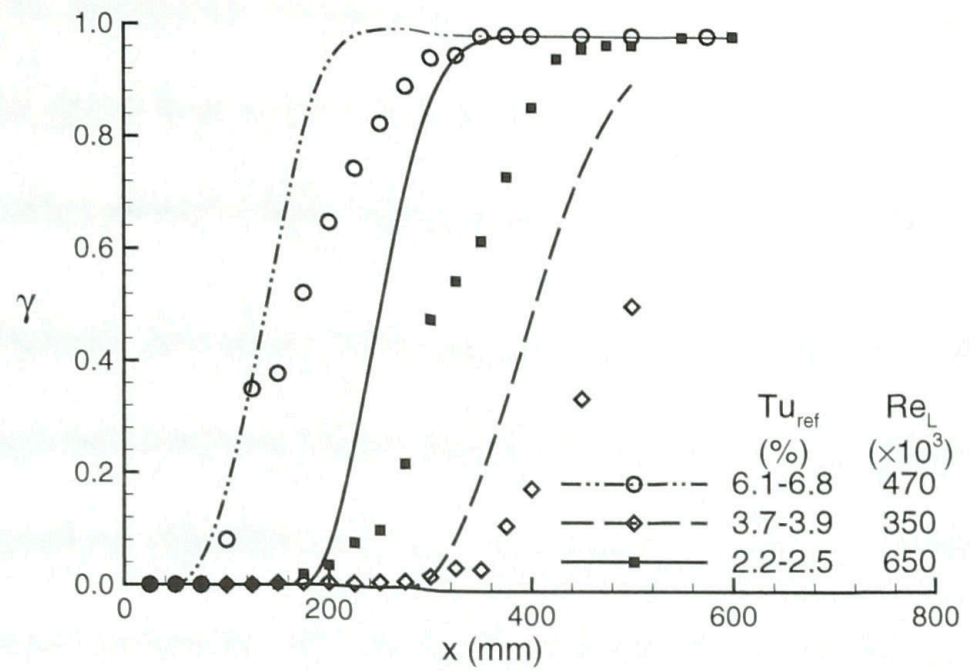

(a) $C_{P 3}$ pressure distribution

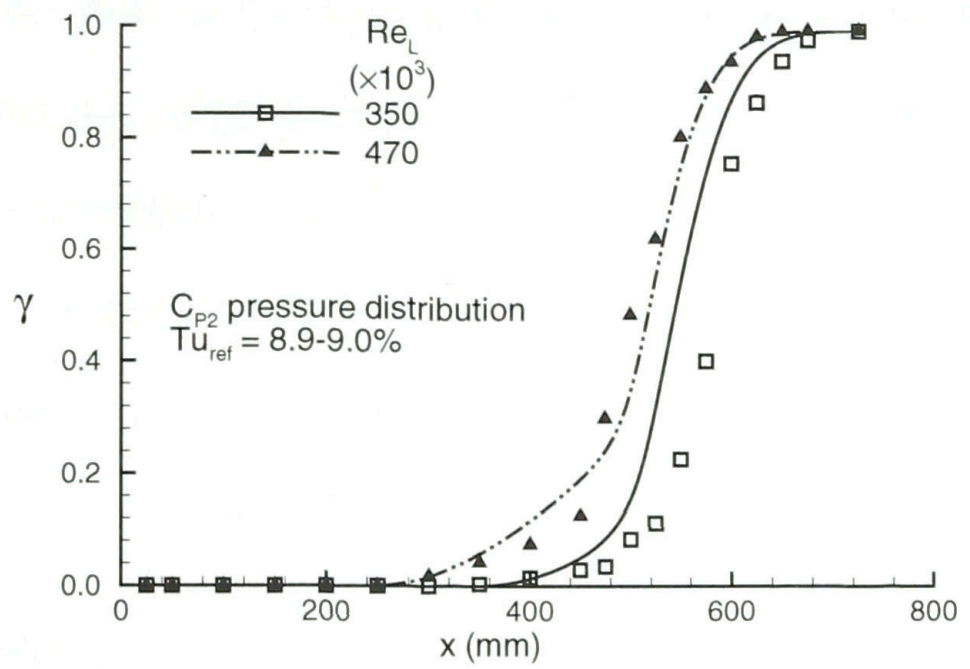

(b) $C_{P 2}$ pressure distribution

Figure 4.11: Comparison of measured intermittency distributions (symbols) to the model of Solomon et al. (1996) (lines) 


\subsection{Summary}

This chapter has presented experimental data on attached flow transition corresponding to systematic variations in the free-stream turbulence intensity and length scale, for up to four values of flow Reynolds number, and three variations of streamwise pressure distributions imposed upon the boundary layer.

For turbulence intensities below about $2 \%$, the momentum thickness Reynolds number at transition inception varies with both free-stream turbulence intensity and the pressure gradient described by $\lambda_{\theta}$. For higher turbulence intensities, $\operatorname{Re}_{\theta_{t s}}$ is judged to depend primarily on the level of free-stream turbulence. However, a significant effect of the turbulence length scale is also observed in several test cases, and length scales closer to the local boundary layer thickness are seen to be more effective at promoting transition inception. In most instances, the observed transition inception point corresponds reasonably well to the prediction of Abu-Ghannam \& Shaw (1980). For the test cases where transition inception occurs at a value of below the stability limit, the transition inception locations in general correspond more closely to the correlations of Mayle (1991), and of Hourmouziadis (1989).

In contrast to the trends seen in the transition inception location, the rate of transition is noted to be primarily dependent on the local pressure gradient parameter.

Although the transition model of Solomon et al. (1996) represents a significant improvement over its predecessors, its prediction accuracy is shown to be inconsistent due to the uncertainty in the predicted turbulent spot production rates. An improved 
spot production rate correlation is therefore required to achieve satisfactory prediction of the measured intermittency distributions. Such a model will be presented in Chapter 8 . 


\section{Chapter 5}

\section{Effects of Free-Stream Turbulence and Periodic Unsteadiness on Separation Bubble Transition}

The effects of periodic unsteadiness on boundary layer transition have recently been the focus of many research programs. In many of these studies, the periodic unsteadiness has been generated by the use of a periodic wake generator upstream of the test surface (e.g. Dong \& Cumpsty, 1990-b; Schobeiri \& Radke, 1994; Solomon \& Walker, 1995), or by performing tests on rotating rigs (e.g. Addison \& Hodson, 1990a,--b; Pullan, 2004). These arrangements result in reasonably accurate simulations of the conditions present in gas turbine engines. However, it is not possible under these conditions to separate the effects of free-stream velocity variations, caused by the momentum deficit in the blade wakes, from those of periodic variations in the turbulence levels. In a study by Lou \& Hourmouziadis (2000), the mean flow fluctuations were generated by periodic blockage of the flow downstream of the test surface, which allows for study of the boundary layer development in the absence of 
periodic variations in free-stream turbulence. In their study, Lou \& Hourmouziadis (2000) examined the effects of periodic free-stream unsteadiness on the separation bubble transition process under low turbulence conditions. In the current work, the effect of periodic variations in the free-stream velocity on separation bubble transition is documented under low turbulence conditions, and at a turbulence level that is more representative of those found on the suction surfaces of low pressure turbine blades. In this fashion, the relative importance of periodic variations in free-stream velocity and turbulence intensity on the transition process in separation bubbles is quantified.

It is important to emphasize that the combined presence of free-stream velocity variations, in the form of standing waves over the test plate, and freestream turbulence considered in the present study is not intended to reproduce the flow physics associated with the time-periodic passing of wakes with momentum deficit and high turbulence. In the latter situation, the turbulence level varies periodically along with the variation in velocity. The presence of a calmed region in the boundary layer immediately following the passing of a wake, as documented by many researchers (e.g. Dong \& Cumpsty, 1990-b; Schobeiri \& Radke, 1994; Solomon \& Walker, 1995; Addison \& Hodson, 1990-a,-b), is likely linked to this periodic variation of turbulence. As a result, such a region would not be observed in the current experiments. Nonetheless, by documenting the relative extent of the effects of periodic velocity variations and free-stream turbulence on the transition process inside separation bubbles, the present study provides indirect evidence on the relative significance of these two factors for the case of boundary layer transition under the periodic influence of wakes. 


\subsection{Test Matrix}

To establish the interaction between the effects of free-stream periodic velocity variations and turbulence on separation bubble transition, measurements were performed at two levels of free-stream turbulence, and three values of unsteadiness frequency. The oscillation frequencies corresponded to Strouhal numbers of $\mathrm{Sr}=0.0$, 2.4, and 4.0 with the Strouhal number defined as:

$$
S r=\frac{f L}{\bar{U}_{r e f}}
$$

The length of the test plate, $L$, is used as the length scale in the Strouhal number, for consistency with other published studies using similar experimental setups. However, replacement of the chord length, used in the definition of $\mathrm{Sr}$ in studies involving airfoils, with the length of the test plate in the current configuration is not quite correct. This is because the pressure distribution imposed on the test surface, and the length of the test plate can be controlled independently. The distance of the point of separation from the leading edge, $x_{s}$, or an integral thickness of the boundary layer at that point, may be argued to be fundamentally more suitable length scales for $S r$. Based on the $x_{s}$ value of about $500 \mathrm{~mm}$ for the present test cases, the non-zero values of the Strouhal number are about 1.0 and 1.6. This range compares reasonably well with those encountered in typical turbomachinery blade rows (approx. 0.6-1.2). These values are also close to the Strouhal numbers of about 0.4 to 1.25 that have been investigated by other researchers (e.g. Schobeiri \& Radke, 
1994; Addison \& Hodson, 1990-a,--b; Lou \& Hourmouziadis, 2000).

In order to establish the Reynolds number sensitivity of the effects of freestream periodic unsteadiness and turbulence on transition, measurements were performed at two Reynolds numbers, based on the plate length $L=1220 \mathrm{~mm}$, and $\bar{U}_{\text {ref }}$ values of about 4.5 and $6.0 \mathrm{~m} / \mathrm{s}$. In each case, the reference velocity setting was adjusted slightly during the course of the experiments to compensate for flow temperature variations of about $\pm 3^{\circ} \mathrm{C}$. The amplitude of the periodic velocity fluctuations, turbulence intensity, and integral length scale of turbulence for the test cases are summarized in Table 5.1. The tabulated amplitudes of periodic velocity oscillations, $\Delta U$, are based on hot-wire $y$ direction traverses $10 \mathrm{~mm}$ upstream of the test plate leading edge. For each test case, $\Delta U$, and $\bar{U}$ were verified to be uniform along the length of the traverse, which extended from the test plate surface level to the ceiling of the test section. As mentioned earlier, the tabulated values of $\Delta U / \bar{U}$ are similar to those experienced by blades passing through the wakes of upstream blade rows in axial turbomachinery. For a systematic study, such as the present experiments, it would have been desirable to maintain a constant value of $\Delta U / \bar{U}$, while varying the primary variables of the test matrix, i.e. free-stream turbulence intensity, frequency of unsteadiness, and flow Reynolds number. As listed in the table however, $\Delta U / \bar{U}$ decreases as the frequency of unsteadiness is increased from $S r=2.4$ to $S r=4.0$. A similar effect is caused by increasing the flow Reynolds number from $R e_{L}=350,000$ to $R e_{L}=470,000$ at $S r=2.4$. The results of the study must therefore be interpreted in the context of these variations in $\Delta U$. The TF parameter listed in the last column of Table 5.1 is Taylor's turbulence factor (Eq. 
Table 5.1: Free-stream conditions

\begin{tabular}{|c|c|c|c|c|c|}
\hline $\begin{array}{c}T u \\
(\%)\end{array}$ & $\begin{array}{c}R e_{L} \\
\left(\times 10^{3}\right)\end{array}$ & $S r$ & $\begin{array}{c}\Delta U / \bar{U} \\
(\%)\end{array}$ & $\begin{array}{c}\Lambda_{s} \\
(\mathrm{~mm})\end{array}$ & $\begin{array}{c}T F \\
(\%)\end{array}$ \\
\hline 0.6 & 350 & 0.0 & 0 & 7 & 1.7 \\
\hline 0.6 & 350 & 2.4 & 30 & 30 & 1.3 \\
\hline 0.6 & 350 & 4.0 & 4 & 31 & 1.3 \\
\hline 0.4 & 470 & 0.0 & 0 & 70 & 0.7 \\
\hline 0.4 & 470 & 2.4 & 8 & 110 & 0.6 \\
\hline 0.4 & 470 & 4.0 & 3 & 120 & 0.6 \\
\hline 4.2 & 350 & 0.0 & 0 & 28 & 8.9 \\
\hline 4.5 & 350 & 2.4 & 30 & 30 & 9.4 \\
\hline 3.8 & 350 & 4.0 & 4 & 28 & 8.1 \\
\hline 3.4 & 470 & 0.0 & 0 & 13 & 8.4 \\
\hline 3.5 & 470 & 2.4 & 8 & 19 & 8.0 \\
\hline 3.2 & 470 & 4.0 & 3 & 14 & 7.8 \\
\hline
\end{tabular}

2.12).

Streamwise distributions of time-averaged acceleration parameter $\bar{\eta}$ are shown for each of the test cases in Fig. 5.1. The ceiling configuration for the test cases presented in this chapter corresponds to the $C_{P 1}$ pressure distribution identified in Section 4.1. These $\bar{\eta}$ distributions are noted to be relatively insensitive to variations in $T u, R e_{L}$, and $S r$.

In the current unsteady free-stream test cases, the transition inception point is non-stationary due to the effects of the mean flow velocity fluctuations. Thus, unlike the results presented in Chapter 4, the first streamwise measurement station at which turbulent spots are observed is not a reliable indication of the time-averaged transition inception location. Therefore, in this chapter, $\overline{x_{t s}}$ is considered to be the first position at which the time-averaged intermittency rate $(d \bar{\gamma} / d x)$ is deemed, visually, to assume a distinctly positive slope. 


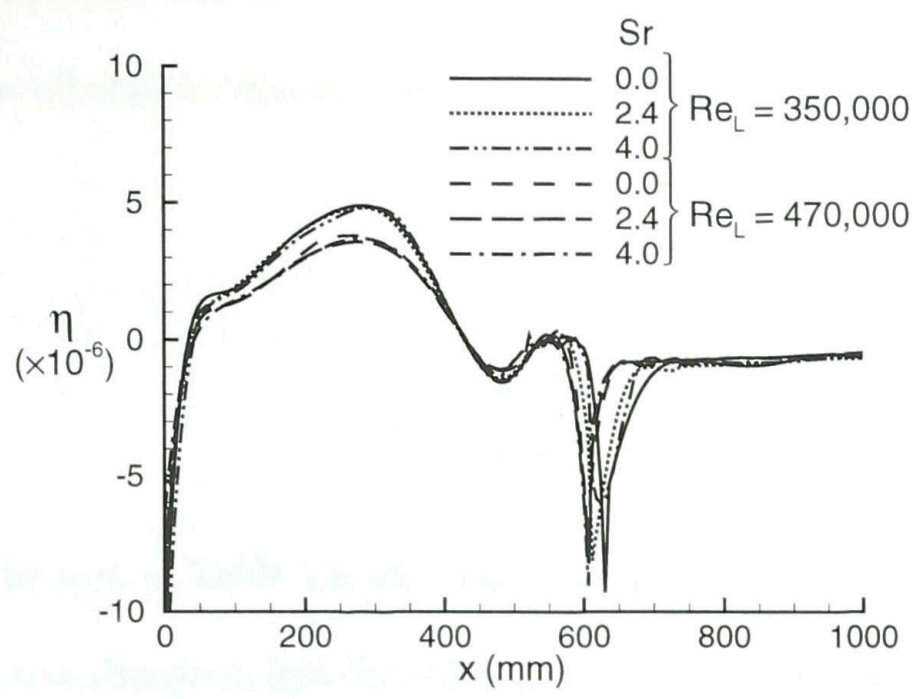

(a) $T u_{\text {ref }}=0.4-0.6 \%$

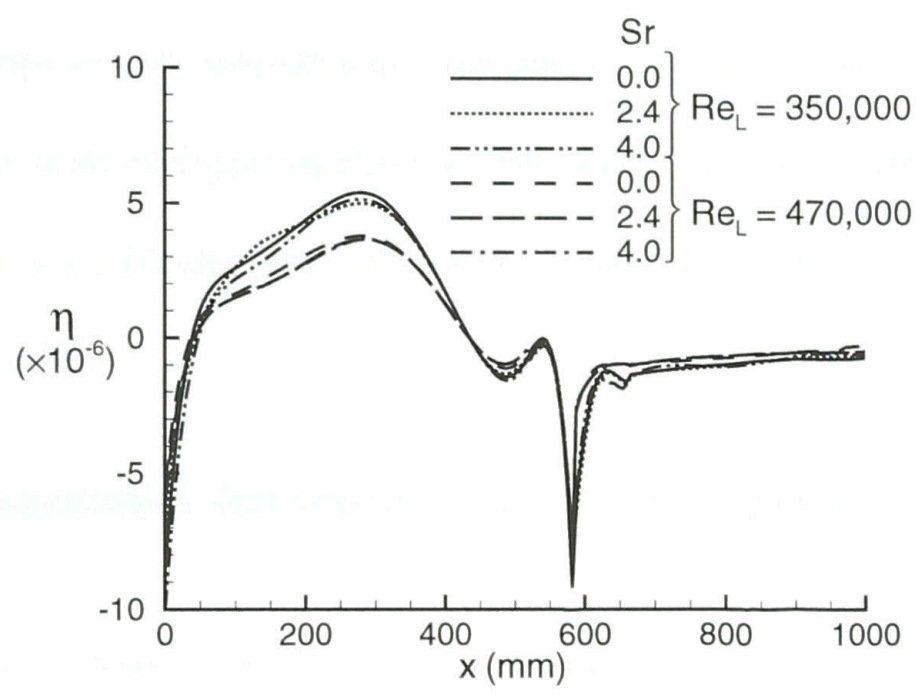

(b) $T u_{\text {ref }}=3.2-4.5 \%$

Figure 5.1: Streamwise variation in acceleration parameter

\subsection{Results and Discussion}

\subsubsection{Transition Inception}

The separation and reattachment points, the locations of transition inception and completion, and a number of flow parameters at the point of separation that 
are known to influence the development of the boundary layer are summarized in Table 5.2. The tabulated time-averaged pressure gradient parameter, $\overline{\lambda_{\theta}}$, is defined as follows:

$$
\overline{\lambda_{\theta}}=\frac{\bar{\theta}^{2}}{\nu} \frac{d \overline{U_{e}}}{d x}
$$

As can be seen in Table 5.2, the time-averaged transition inception point $\left(\overline{x_{t s}}\right)$ is not sensitive to a change in flow Reynolds number from 350,000 to 470,000. A small effect of Strouhal number on the transition inception location is noted, which is limited to the conditions of low free-stream turbulence. This movement of the transition inception point with Strouhal number is observed to be very small in comparison to that associated with an increase in turbulence intensity from $T u_{\text {ref }}=0.4-0.6 \%$ to $T u_{r e f}=3.2-4.5 \%$.

\subsubsection{Streamwise Intermittency Distributions}

The time-averaged intermittency distributions calculated at a distance of $1.25 \mathrm{~mm}$ from the surface of the test plate are presented in Fig. 5.2. The results in the figure are presented in groups corresponding to the same flow Reynolds number and turbulence intensity. Thus, the differences seen in the intermittency distributions on each plot in this figure are due solely to the effects of the periodic unsteadiness of the free-stream.

In Fig. 5.2, the intermittency distributions are noted to be very similar for $S r=0.0$ and $S r=4.0$. The similarity in the transition process for these two Strouhal 


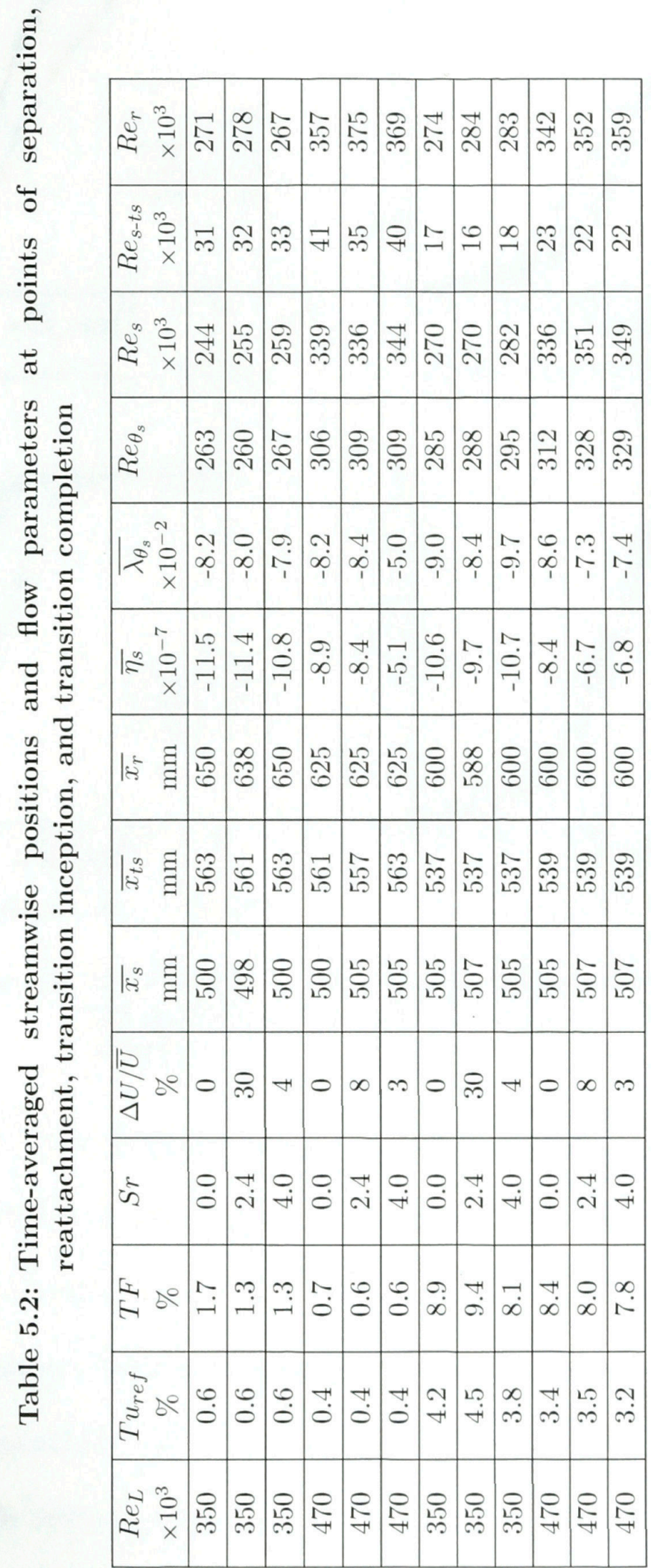




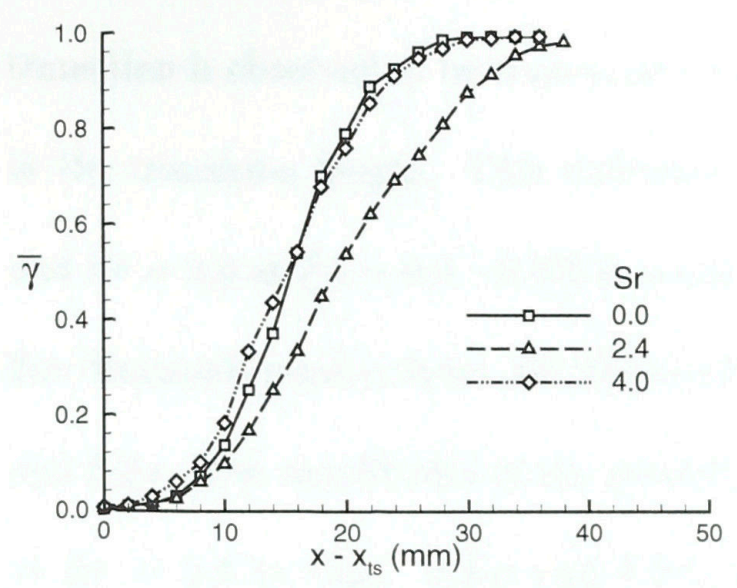

(a) $T u=0.4-0.6 \%, R e_{L}=350,000$

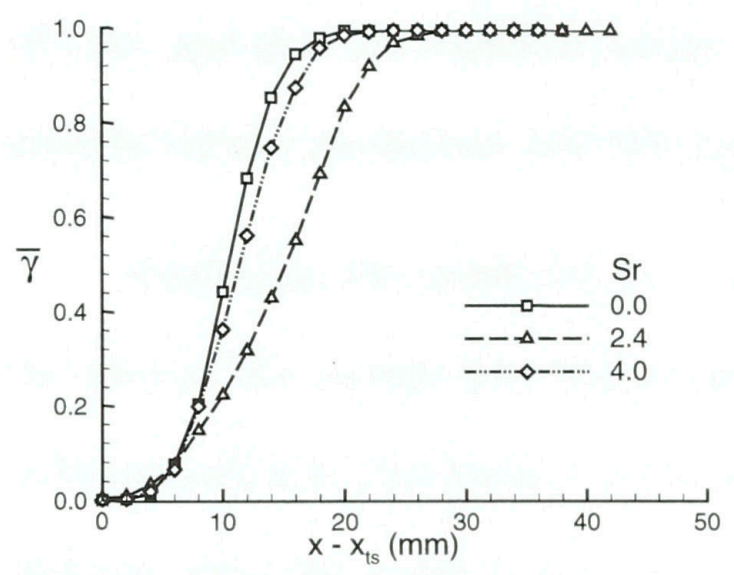

(c) $T u=0.4-0.6 \%, R e_{L}=470,000$

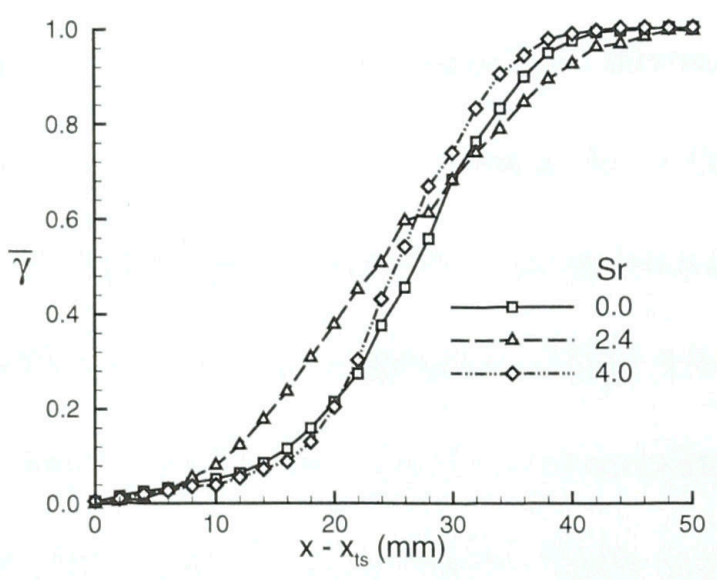

(b) $T u=3.2-4.5 \%, R e_{L}=350,000$

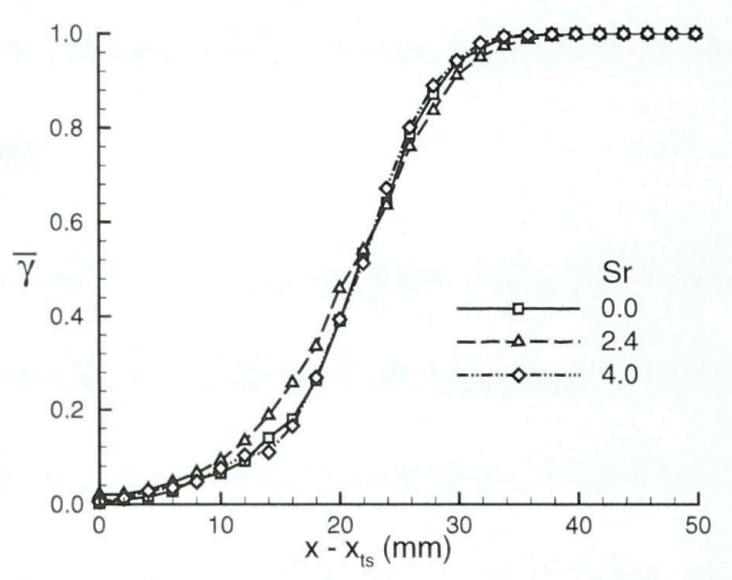

(d) $T u=3.2-4.5 \%, R e_{L}=470,000$

Figure 5.2: Variation in the time-averaged streamwise intermittency distribution with Strouhal number

numbers is expected for the higher turbulence case, since the amplitude of the periodic

flow oscillations (Table 5.1) is smaller than the rms of the velocity fluctuations due to free-stream turbulence. However, persistence of this similarity at low levels of free-stream turbulence suggests that periodic free-stream velocity unsteadiness at a frequency corresponding to $S r=4.0$ and peak-to-peak amplitude of about 3-4\% of $\bar{U}$ has a negligible effect on the transition process in the separation bubble. 
Comparing the trends for $S r=0.0$ and $S r=2.4$ in Fig. 5.2-a, the rate of transition is observed to be somewhat lower in the latter case, resulting in an increase in the transition length. This difference in the transition length between $S r=0.0$ and $S r=2.4$ at $T u=0.4-0.6 \%$ is noted to be relatively insensitive to an increase in flow Reynolds number from 350,000 to 470,000, as shown by comparison of Figs. 5.2-a and 5.2-c. The amplitudes of the periodic velocity oscillations $\Delta U / \bar{U}$, corresponding to $S r=2.4$ in Figs. 5.2-a and 5.2-c, are 30\% and 8\%, respectively (Table 5.1). Therefore, it may be concluded that the observed difference in the transition rate $(d \bar{\gamma} / d x)$, and thus the transition length, is also insensitive to the amplitude of the periodic velocity oscillations over this range.

Comparing the trends for $S r=0.0$ and $S r=2.4$ in Figs. 5.2-a and 5.2-b, the effect of the periodic free-stream unsteadiness on the rate of transition is noted to be insensitive to free-stream turbulence variations over a range from $0.5 \%$ to $4 \%$. However, when the trends in Figs. 5.2-a and 5.2-d are compared to each other, the difference in transition rate between $S r=0.0$ and $S r=2.4$ observed in Fig. 5.2-a diminishes significantly with an increase in both Reynolds number and turbulence intensity. This may be indicative of interactions between the effects of Reynolds number and free-stream turbulence on transition under periodic-unsteady conditions.

As shown in Fig. 5.3, turbulent spots develop periodically at the points of maximum rate of free-stream deceleration in each oscillation cycle for the $T u_{\text {ref }}=0.4-0.6 \%$ test cases at $S r=2.4$. This phenomenon, also reported by Lou \& Hourmouziadis (2000), was not observed for any of the test cases at 


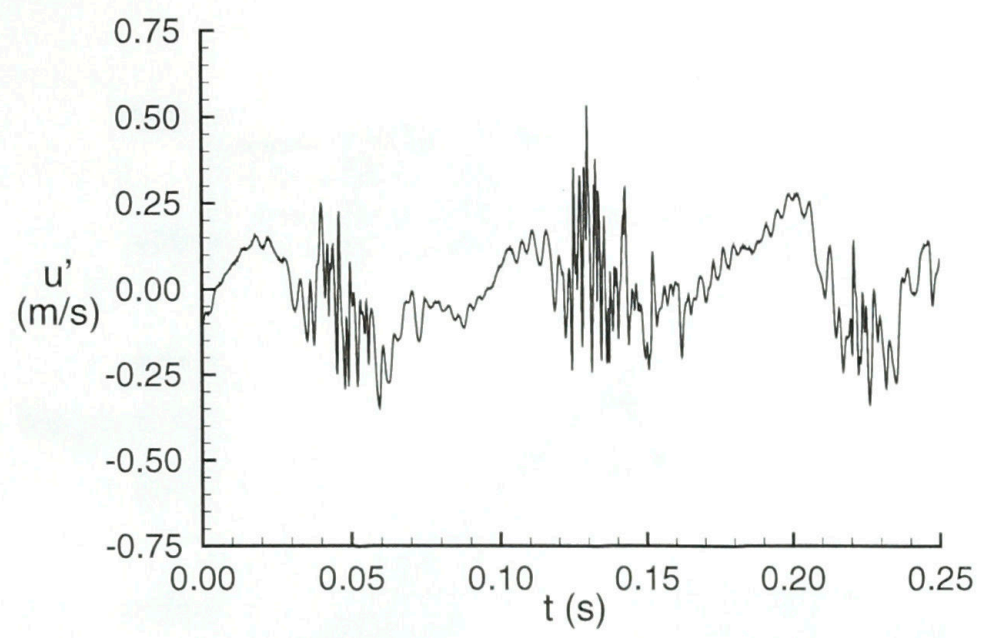

Figure 5.3: Periodic turbulent spot development in the boundary layer $\left(T u_{r e f}=0.4-0.6 \% ; S r=2.4\right)$

$T u_{\text {ref }}=3.2-4.5 \%$, or at $S r=4.0$. Since the amplitude of periodic velocity variations at $S r=4.0$ were notably lower than those at $S r=2.4$ (Table 5.1), it may also be concluded that the coupling between periodic velocity oscillations and transition inception is reduced with increased frequency and reduced amplitude of the periodic free-stream unsteadiness. Since the changes in the frequency and amplitude of the free-stream unsteadiness took place simultaneously, it is not possible to identify the relative significance of these two factors on the coupling between periodic unsteadiness and transition inception based on the present test cases.

\subsubsection{Comparison to Existing Models}

In Figure 5.4, the Reynolds numbers based on the distances of the transition inception and reattachment points from the leading edge are compared to the correlations of Hatman \& Wang (1999) and Yaras (2002). In this figure, the symbols filled in black represent the steady flow $(S r=0.0)$ measurements, and 


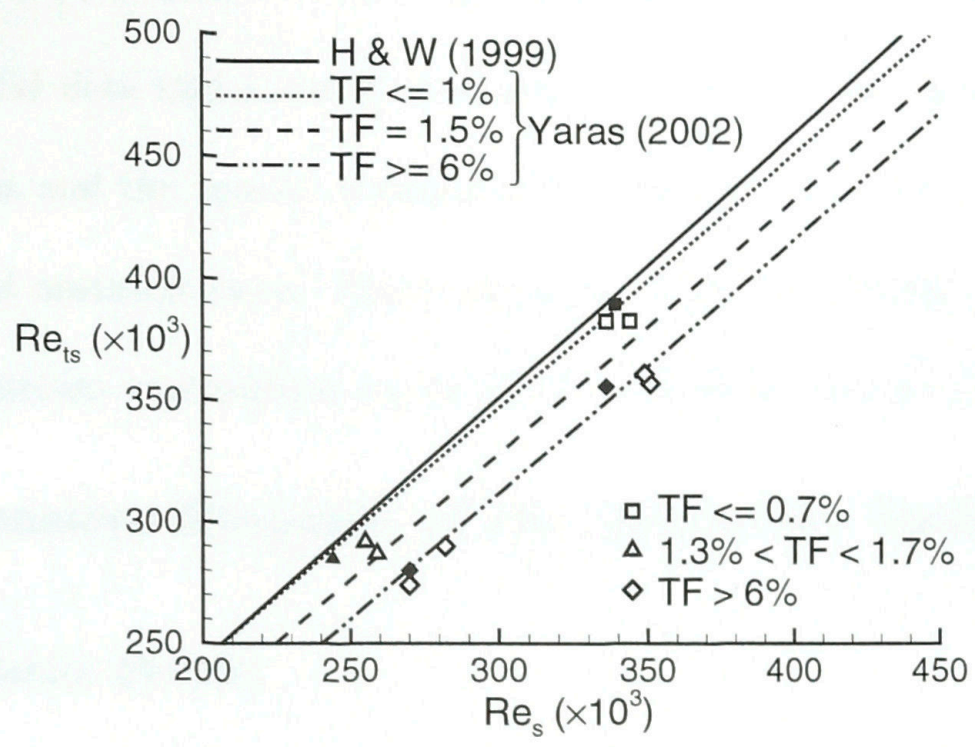

(a) $R e_{t s}$

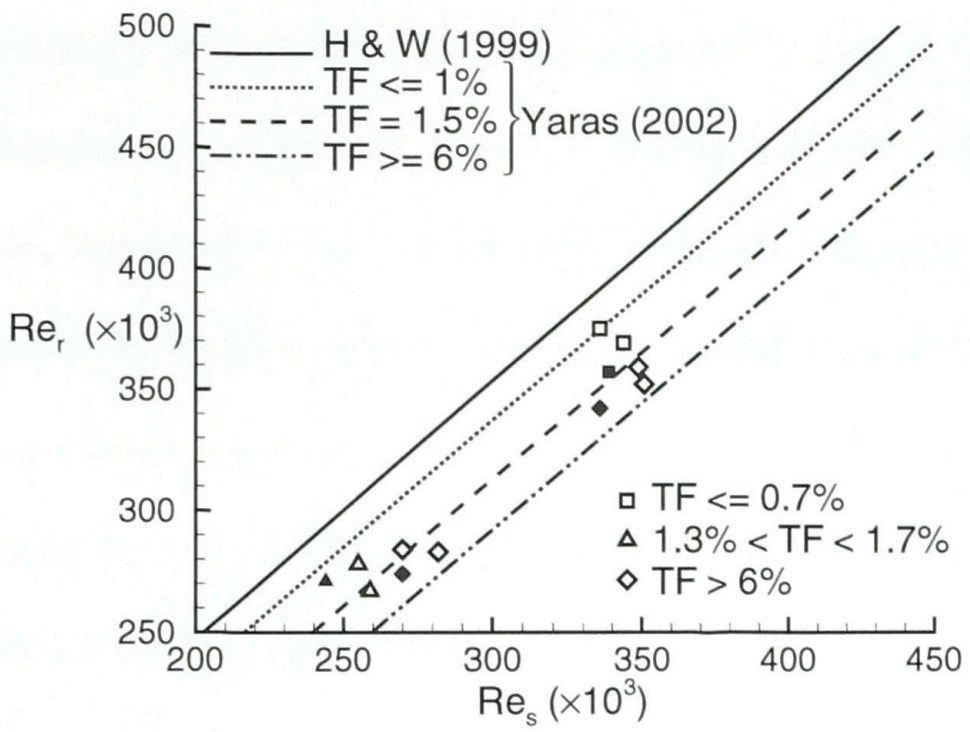

(b) $R e_{r}$

Figure 5.4: Observed experimental variations in $R e_{t s}$ and $R e_{r}$ with $R e_{s}$, compared to the predictions of Hatman \& Wang (H \& W, 1999) and Yaras (2002) (Hollow symbols: periodic-unsteady measurements; Filled symbols: steady-flow measurements) 
the hollow symbols represent the measurements taken under periodic-unsteady flow conditions $(S r=2.4$ and $S r=4.0)$. Although these models were developed based on experimental data under steady free-stream conditions, the agreement between the predictions and the present experimental results are observed to be similar for the steady and unsteady cases. This is consistent with the relatively small effects of periodic free-stream unsteadiness on the transition process noted in Fig. 5.2.

\subsubsection{Transient Response of the Separation Bubble}

\subsubsection{Velocity Profiles}

In Figs. 5.5 and 5.6, the ensemble-averaged velocity profiles near and within the separation bubble are presented for each of the test cases at the instances of minimum and maximum free-stream velocity (when applicable), with each figure representing measurements at one of the flow Reynolds numbers. The dividing streamline is traced by fitting a polynomial curve through the points in the velocity profiles where a notable increase in velocity is first measured. The reattachment point is estimated by the streamwise position at which the velocity profile most closely resembles Horton's (1968) "universal" reattachment profile. The trends in Figs. 5.5 and 5.6 clearly show a reduction in the thickness of the separation bubble with increasing turbulence intensity and Reynolds number. Comparing the results in Fig. 5.5 to those in Fig. 5.6, at low free-stream turbulence, an increase in Reynolds number from 350,000 to 470,000 reduces the thickness of the separation bubble by approximately one-third. Comparing the trends between columns of these figures, an increase in turbulence intensity from approximately 0.5 to $4 \%$ leads to a reduction in 


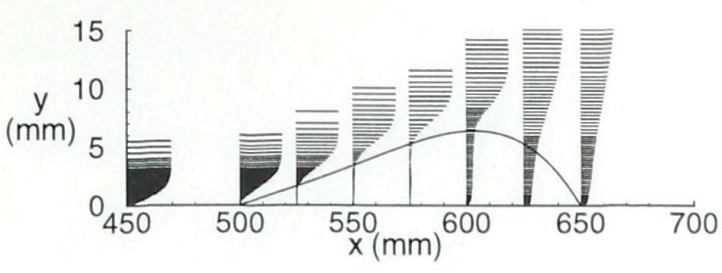

(a) $\mathrm{Tu}=0.4-0.6 \% ; \mathrm{Sr}=0.0$
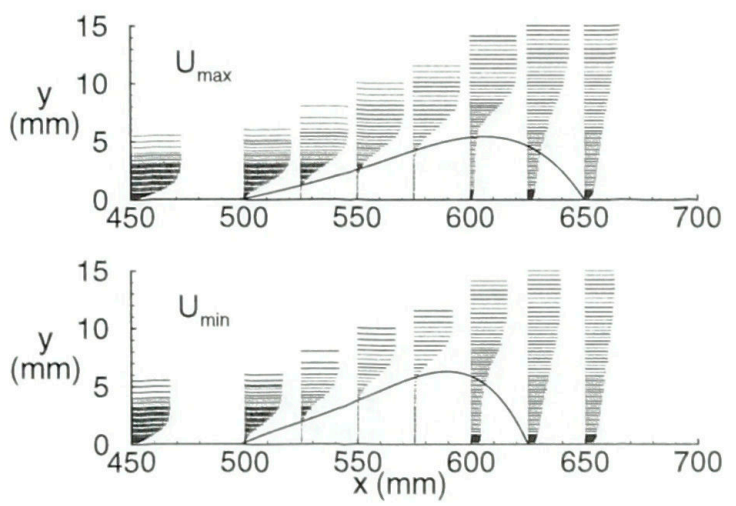

(b) $T u=0.4-0.6 \% ; S r=2.4$
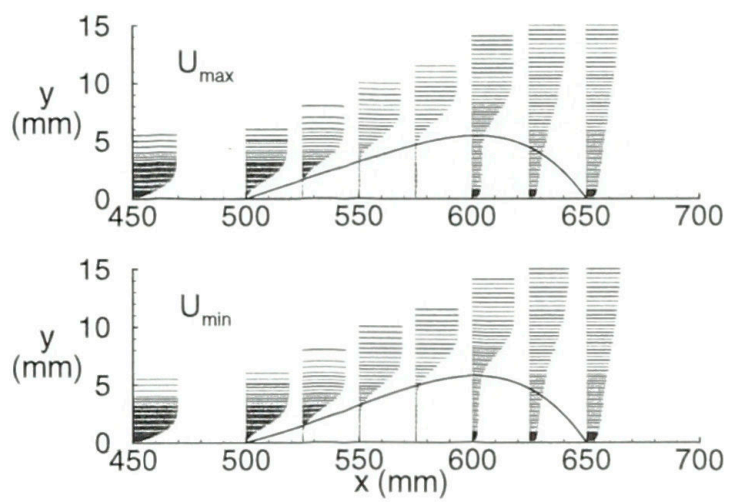

(c) $\mathrm{Tu}=0.4-0.6 \% ; \mathrm{Sr}=4.0$

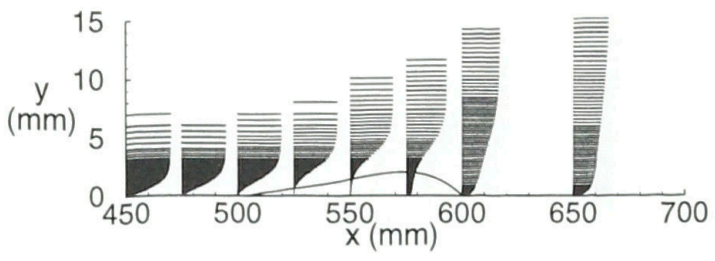

(d) $T u=3.2-4.5 \% ; S r=0.0$
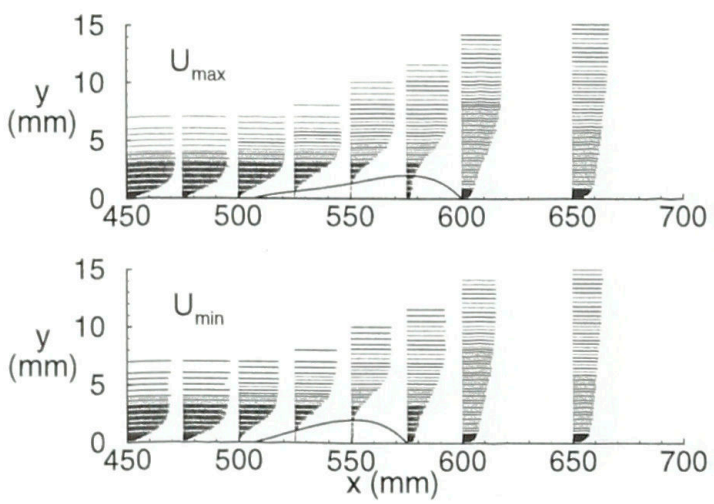

(e) $\mathrm{Tu}=3.2-4.5 \% ; \mathrm{Sr}=2.4$
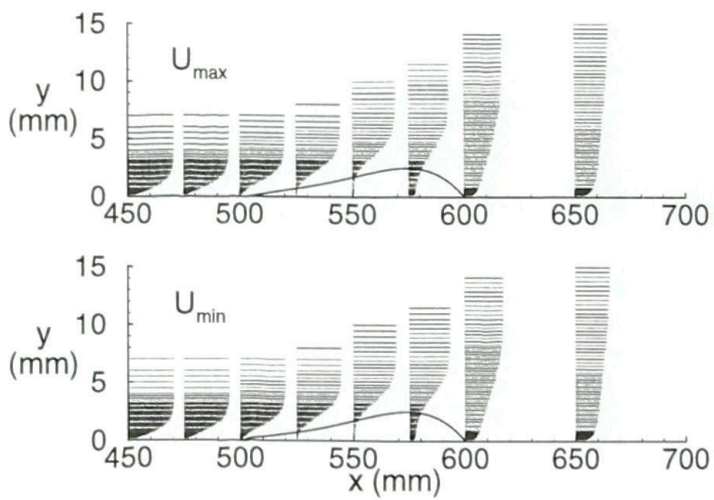

(f) $\mathrm{Tu}=3.2-4.5 \% ; \mathrm{Sr}=4.0$

Figure 5.5: Ensemble-averaged velocity profiles at the phases of maximum and minimum free-stream velocity $-R e_{L}=350,000$ (estimated separation bubble dimensions also shown)

the bubble thickness of approximately 50\%. However, there does not appear to be a significant change in the separation bubble thickness with Strouhal number, since the bubble heights are approximately the same for a given Reynolds number and turbulence intensity. In addition, there does not seem to be a significant effect of the periodic unsteadiness on the location of separation, as was also noted by Lou \& 


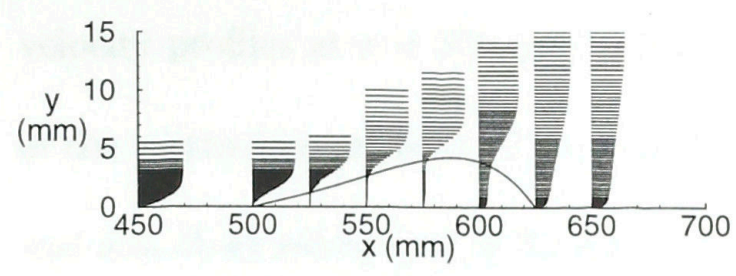

(a) $\mathrm{Tu}=0.4-0.6 \% ; \mathrm{Sr}=0.0$
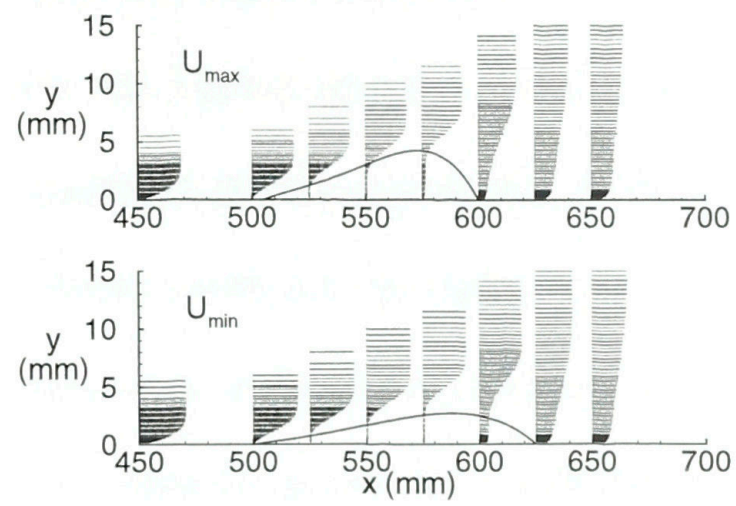

(b) $T u=0.4-0.6 \% ; S r=2.4$
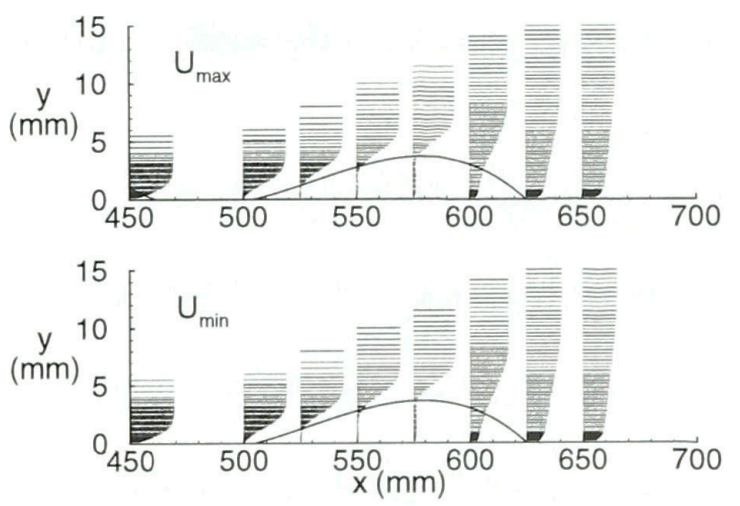

(c) $\mathrm{Tu}=0.4-0.6 \% ; \mathrm{Sr}=4.0$

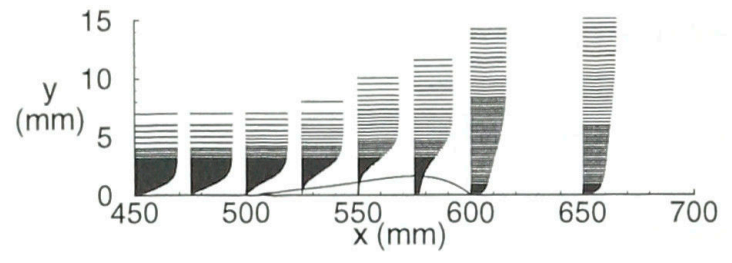

(d) $T u=3.2-4.5 \% ; S r=0.0$
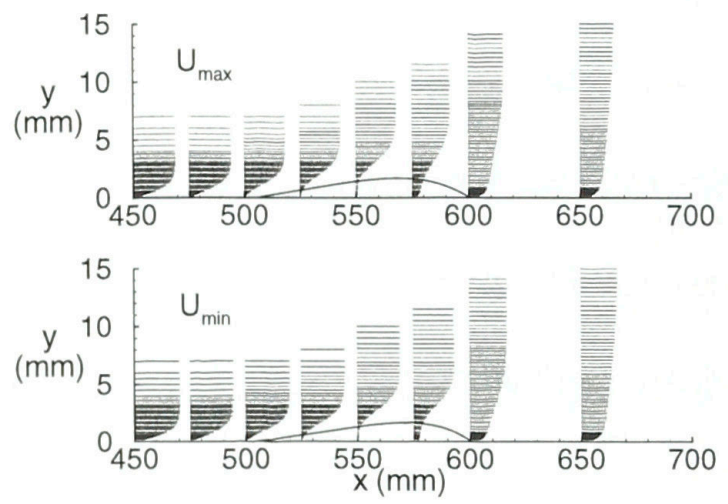

(e) $T u=3.2-4.5 \% ; S r=2.4$
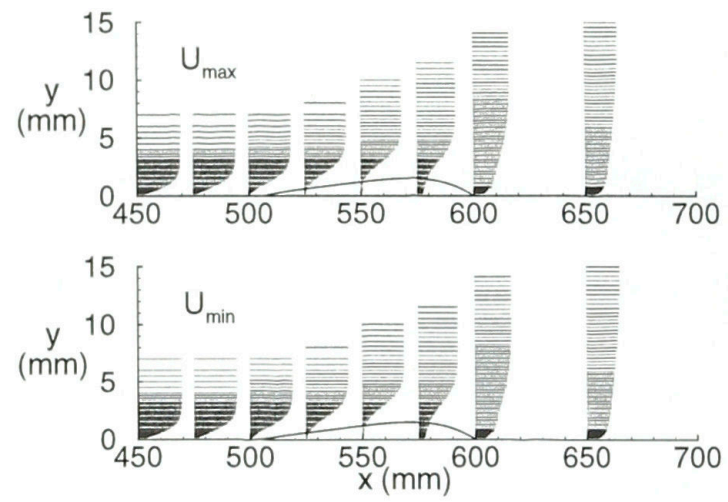

(f) $T u=3.2-4.5 \% ; S r=4.0$

Figure 5.6: Ensemble-averaged velocity profiles at the phases of maximum and minimum free-stream velocity $-R e_{L}=470,000$ (estimated separation bubble dimensions also shown)

Hourmouziadis (2000).

It may be concluded from these figures however, that the mean flow unsteadiness in some instances causes a periodic movement of the reattachment point, with the reattachment point moving upstream as the free-stream velocity is decreased during the oscillation cycle. This is most notable in Figs. 5.5-b and 5.5-e, although the 
velocity profiles at $x=600 \mathrm{~mm}$ in Fig. 5.6-b also suggest a small periodic movement of the reattachment point. This periodic movement of the reattachment point, which was also observed by Lou \& Hourmouziadis (2000), is seen to decrease with increasing Reynolds number and turbulence intensity, as evidenced by the trends in the figures. For the highest Strouhal number (Figs. 5.5-c, 5.5-f, 5.6-c and 5.6-f), there is no significant periodic movement of the reattachment point. This is evident from the velocity profiles at the maximum and minimum velocity phases of the oscillation cycle being very similar to each other, as well as to the velocity profiles measured under the respective steady flow conditions (Figs. 5.5-a \& -d and 5.6-a \& -d).

\subsubsection{Boundary Layer Integral Parameters}

The streamwise distributions of the ensemble-averaged displacement thickness corresponding to the velocity profiles presented in Figs. 5.5 and 5.6 are given in Figs. 5.7 and 5.8 for $R e_{L}=350,000$ and $R e_{L}=470,000$, respectively. The point of maximum displacement thickness occurs near the point of transition completion for the cases at low turbulence levels, and corresponds approximately to the midtransition point for the cases at higher turbulence intensity.

The distributions of $\delta^{*} U_{\min }$ and $\delta^{*}{ }_{\max }$ in Fig. 5.7-a, which correspond to the maximum and minimum free-stream velocity phases of the oscillation period respectively, indicate that the response of the separation bubble to free-stream unsteadiness is not quasi-steady. Upstream of the point of maximum displacement thickness, the ensemble-averaged displacement thickness of the separated boundary layer is notably greater at the instant of lower free-stream velocity. This is consistent 


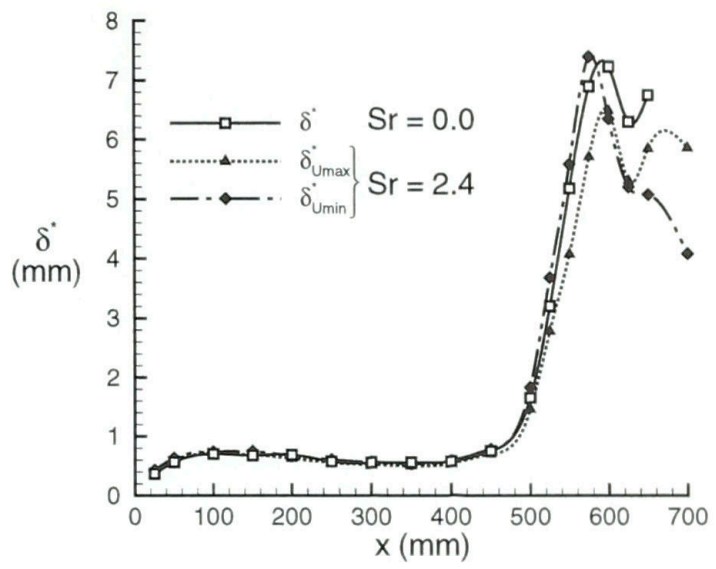

(a) $T u=0.4-0.6 \%$

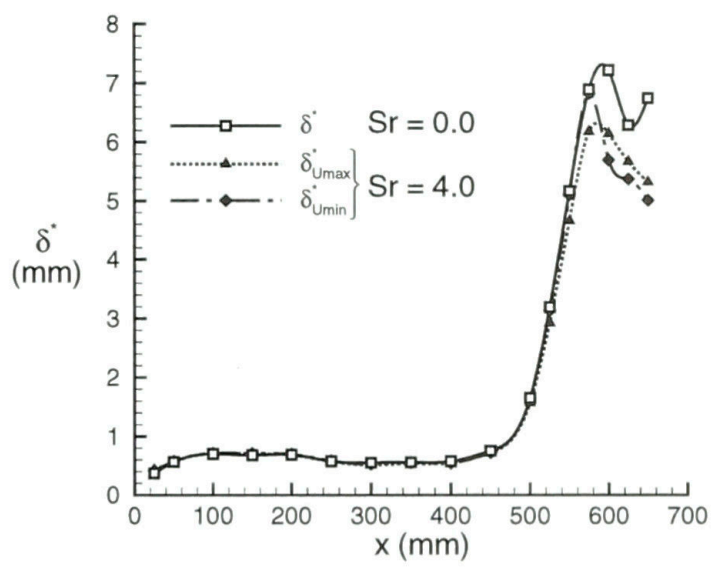

(c) $T u=0.4-0.6 \%$

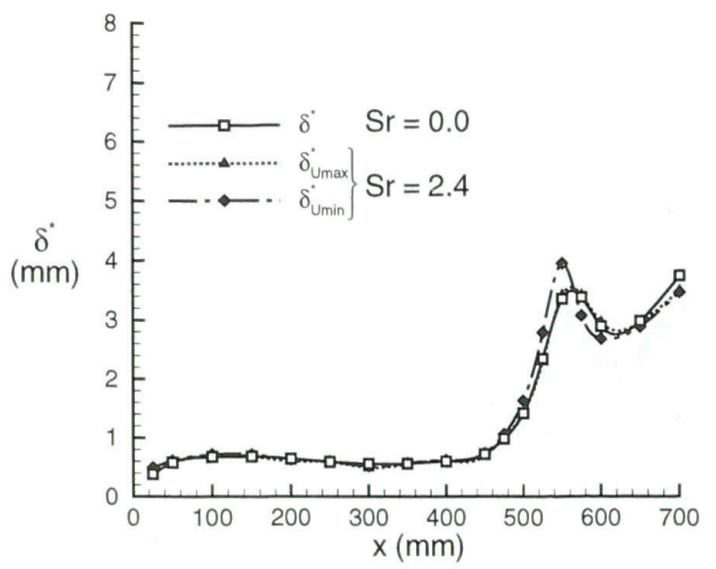

(b) $T u=3.2-4.5 \%$

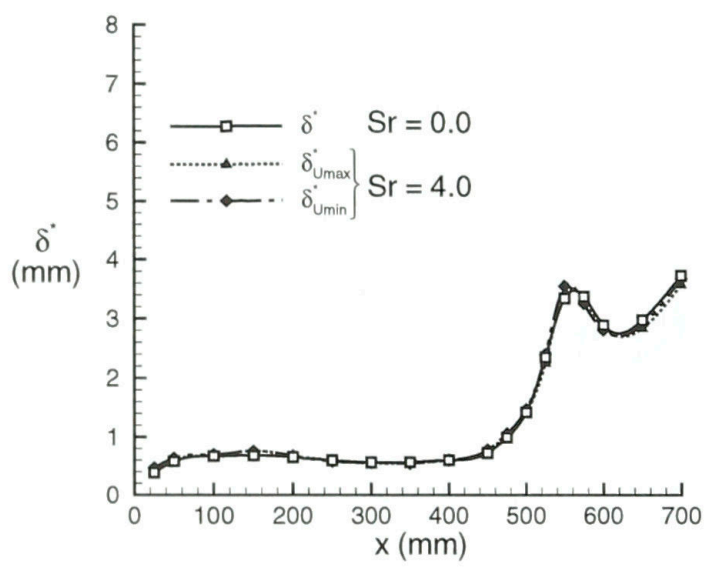

(d) $T u=3.2-4.5 \%$

Figure 5.7: Streamwise variation of steady $(S r=0.0)$ and ensemble-averaged $(S r=2.4 \& 4.0)$ displacement thickness at the maximum and minimum free-stream velocity phases $-R e_{L}=$ 350,000

with the trend in $\delta^{*}$ that would be expected if the flow Reynolds number were gradually varied. However, this consistency with quasi-steady behaviour is true only in a qualitative sense. This is because the relative magnitudes of $\delta^{*} U_{\min }$ and $\delta^{*} U_{\max }$ in comparison to the $\delta^{*}$ values for steady free-stream conditions, also shown in the figure, 


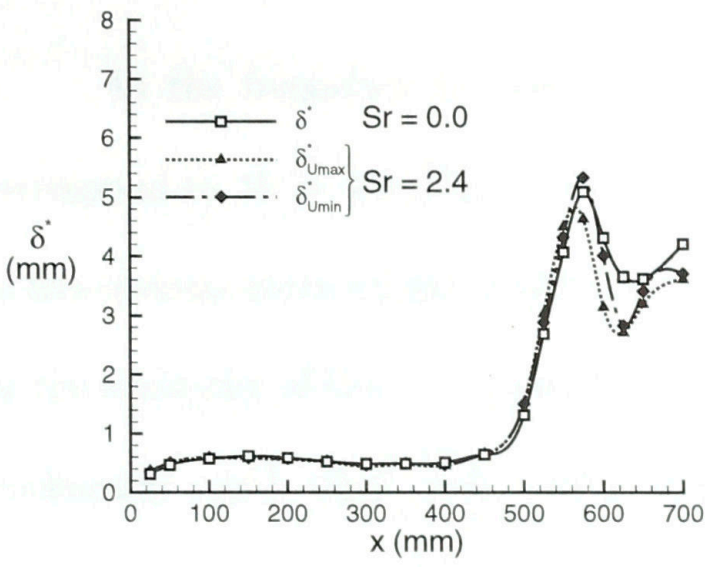

(a) $\mathrm{Tu}=0.4-0.6 \%$

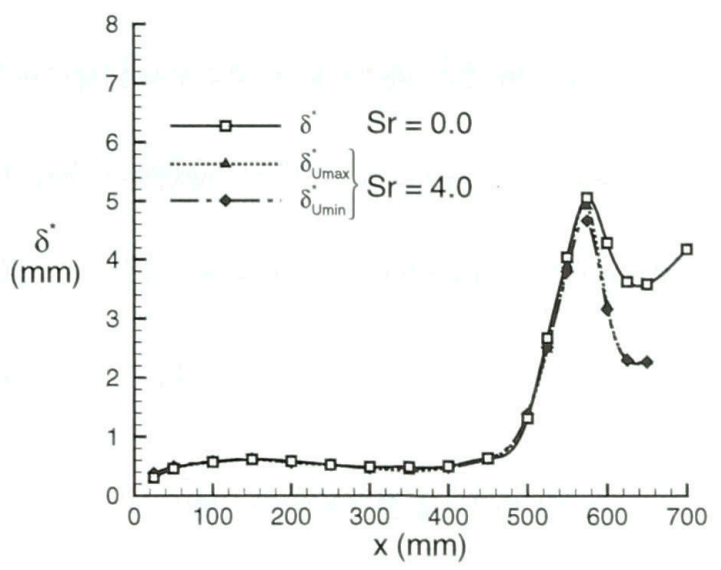

(c) $\mathrm{Tu}=0.4-0.6 \%$

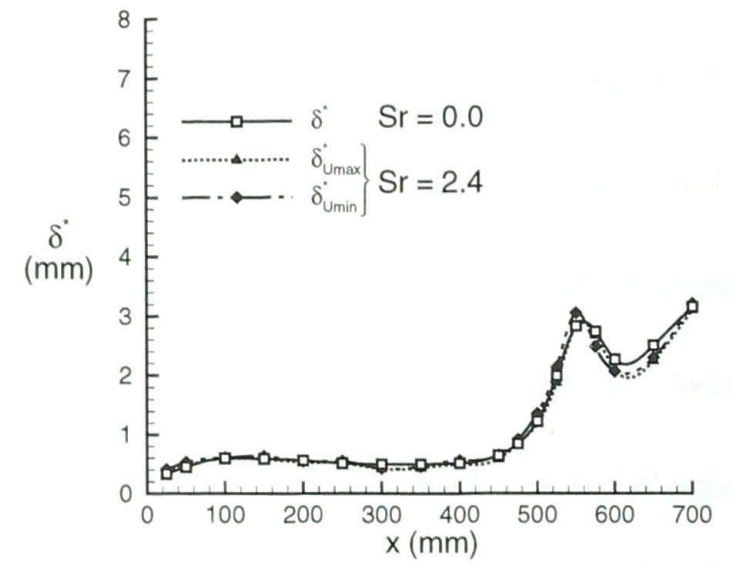

(b) $T u=3.2-4.5 \%$

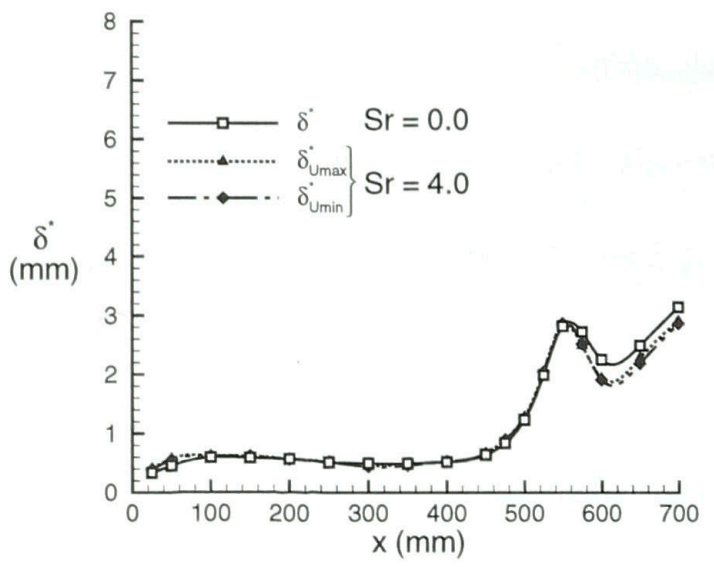

(d) $T u=3.2-4.5 \%$

Figure 5.8: Streamwise variation of steady $(S r=0.0)$ and ensemble-averaged $(S r=2.4 \& 4.0)$ displacement thickness corresponding to the maximum and minimum free-stream velocity phases $-\operatorname{Re}_{L}=470,000$

are not those that would be expected for quasi-steady flow. As noted earlier, the timeaveraged locations of separation are observed to correspond closely to the separation point under steady free-stream conditions. Downstream of the point of maximum $\delta^{*}$, the response of the separated shear layer to unsteady free-stream conditions is not quasi-steady even in a qualitative sense. This is demonstrated by the mutual crossing 
of the $\delta^{*} U_{\min }, \delta^{*}{ }_{U_{\max }}$, and steady flow $\delta^{*}$ trends.

As the frequency of free-stream periodic velocity variations is increased to correspond to $\mathrm{Sr}=4.0$ (Fig. 5.7-b), the transient response of the separation bubble to free-stream unsteadiness is observed to be less pronounced. This is demonstrated by the similarity of the $\delta^{*} U_{\min }$ and $\delta^{*}{ }_{U_{\max }}$ distributions up to the location where these thicknesses reach their peak, and a smaller difference between these distributions beyond the peaks, compared to that observed for $S r=2.4$ in Fig. 5.7-a. While it is expected that the separation bubble would follow low frequency free-stream fluctuations more readily than high frequency fluctuations, the diminished response of the bubble to free-stream oscillations observed in Fig. 5.7-b is also partly due to the notably lower amplitude of the free-stream velocity oscillations $(\Delta U / \bar{U}=4 \%$ vs. $\Delta U / \bar{U}=30 \%$ in Fig. 5.7-a).

As the free-stream turbulence is increased (Fig. 5.7-a vs. 5.7-c, and Fig. 5.7-b vs. 5.7-d), the differences between the $\delta^{*}{ }_{U_{\min }}, \delta^{*}{ }_{U_{\max }}$, and steady $\delta^{*}$ distributions diminish. This is particularly true in the case of $S r=4.0$ (Fig. 5.7-d), where the effects of the free-stream unsteadiness are nearly absent. This is expected since the peak-to-peak amplitude of the periodic free-stream variations in these instances are less than the rms velocity fluctuations associated with the free-stream turbulence.

The effect of increasing the flow Reynolds number from 350,000 to 470,000 on the separation bubble, in terms of the bubble's response to free-stream unsteadiness, is similar to the effect of increasing the free-stream turbulence from about $0.5 \%$ to $4 \%$ (Fig. 5.7 vs. Fig. 5.8). It is also observed that with the combined effects of 
increased flow Reynolds number and free-stream turbulence, the effect of free-stream unsteadiness on the separation bubble is not significant (Figs. 5.8-c and 5.8-d).

\subsection{Summary}

The trends in transitional separation bubbles presented in this chapter give an indication of the relative importance of periodic free-stream velocity fluctuations, free-stream turbulence intensity, and flow Reynolds number. The ranges considered for each of these parameters are similar to those of low pressure turbine blades periodically influenced by the wakes of upstream blade rows. The present results suggest that the amplitude and frequency of periodic velocity fluctuations are less critical to the transition process than the flow Reynolds number, and the variations in turbulence caused by these wakes.

The results demonstrate that the time-averaged locations of flow separation, transition inception, and reattachment are insensitive to periodic velocity oscillations over the ranges of frequency and amplitude considered. This is true for all values of the free-stream turbulence intensity and flow Reynolds numbers tested. In addition, the time-averaged rate of transition is found to be very similar for $S r=0.0$ and $S r=4.0$. For $S r=2.4$ however, the transition rate is lower. This difference between $S r=2.4$ and $S r=4.0$ diminishes when both the flow Reynolds number and freestream turbulence are increased, and the difference in $\Delta U / \bar{U}$ for the two Strouhal numbers becomes less pronounced.

The effects of periodic free-stream unsteadiness on the ensemble-averaged 
location of separation and thickness of the separation bubble are found to be negligible. In contrast, the location of re-attachment, and hence transition completion, is observed to oscillate at the lower of the non-zero Strouhal numbers. The extent of this periodic movement is noted to decrease with increasing flow Reynolds number and free-stream turbulence. Through examination of the ensembleaveraged displacement thickness, the response of the bubble to the periodic freestream unsteadiness is shown not to be quasi-steady. 


\section{Chapter 6}

\section{Effects of Surface Roughness and Free-Stream Turbulence on Boundary Layer Transition}

The effects of streamwise pressure gradient, free-stream turbulence, periodicunsteady free-stream velocity, and the interactions between these parameters as they affect transition on turbomachinery blades have been studied extensively. This is evident by the large number of studies dedicated to this subject, as reviewed in Chapter 2. Such studies have formed the basis for models that predict transition in attached flows (e.g. Abu-Ghannam \& Shaw, 1980; Johnson, 1994, 1998; Narasimha, 1985; Solomon et al., 1996), and in separation bubbles (e.g. Yaras, 2002; Hatman \& Wang, 1999; Horton, 1968). However, no models are yet available in the open literature that account for effects of surface roughness on transition in separation bubbles. This portion of the current study has been undertaken to supplement the limited data available on attached flow transition affected by surface roughness, and to establish the effect of surface roughness on separated-flow transition. 
In the present work, the location of inception and rate of transition are documented for seven surfaces with different roughness geometries. The study encompasses ranges of roughness height from 0.7 to $185 \mu \mathrm{m}$, skewness between -0.6 and +0.4 , and spacing from 490 to $2720 \mu \mathrm{m}$. Some of the surfaces were chosen to have the same roughness height but different roughness geometry, allowing for a systematic study of the effects of roughness spacing and skewness on the transition process. Results are presented for three flow Reynolds numbers, three free-stream turbulence levels, and two streamwise pressure distributions, to establish the sensitivity of roughness effects to these parameters.

\subsection{Geometry of the Rough Surfaces}

The test surfaces used in this portion of the study were described briefly in Chapter 3. The smoothest surface $\left(k_{r m s}=0.7 \mu \mathrm{m}\right)$ is the $25 \mathrm{~mm}$ thick CHARTMIC-6 aluminium plate used in the earlier phases of this research project, for which the effects of periodic unsteady flow conditions were investigated (Chapter 5). The leading edge of this test surface is machined to a $15^{\circ}$ knife-edge, with an elliptic tip of $15.9 \mathrm{~mm}$ and $3.2 \mathrm{~mm}$ major and minor axis dimensions. The remaining test surfaces were constructed by bonding materials with different roughness patterns to a $25.4 \mathrm{~mm}$ thick medium density fibre (MDF) board, to which a $50.8 \mathrm{~mm}$ (2 in.) long aluminium attachment with the same leading edge geometry as the smooth plate was fixed. These roughness layers consist of two countertop laminates $\left(k_{r m s}=31\right.$ and $33 \mu \mathrm{m})$, vinyl floor material $\left(k_{r m s}=48 \mu \mathrm{m}\right)$, tar paper $\left(k_{r m s}=53 \mu \mathrm{m}\right)$, fine asphalt shingle $\left(k_{r m s}=107 \mu \mathrm{m}\right)$, and coarse asphalt shingle $\left(k_{r m s}=185 \mu \mathrm{m}\right)$ surfaces. 
Table 6.1: Measured surface roughness parameters

\begin{tabular}{|l|c|c|c|}
\hline Surface & $\begin{array}{c}k_{\text {rms }} \\
(\mu \mathrm{m} \pm 7 \%)\end{array}$ & $\begin{array}{c}W \\
(\mu \mathrm{m} \pm 7 \%)\end{array}$ & $\begin{array}{c}S_{\text {sk }} \\
( \pm 0.1)\end{array}$ \\
\hline Smooth & $0.7^{*}$ & - & - \\
\hline Blue Laminate & 31 & 2720 & +0.4 \\
\hline White Laminate & 33 & 2270 & -0.1 \\
\hline Vinyl & 48 & 3500 & +0.2 \\
\hline Tar Paper & 53 & 490 & +0.4 \\
\hline Fine Shingle & 107 & 540 & 0.0 \\
\hline Coarse Shingle & 185 & 1610 & -0.6 \\
\hline
\end{tabular}

* Roughness height specified by the manufacturer

The geometry of the rough surfaces was measured by laser triangulation, as described in Section 3.1.3. The statistical roughness parameters deemed to have the strongest influence on the flow are summarized in Table 6.1, and are described below. The height of the roughness elements is quantified by the root-mean-square (RMS) roughness height, computed as

$$
k_{r m s}=\sqrt{\frac{1}{N} \sum_{i=1}^{N}\left(y_{i}-\mu\right)^{2}}
$$

where $\mu$ is the mean surface elevation, defined as

$$
\mu=\sum_{i=1}^{N} \frac{y_{i}}{N}
$$

and $N$ is the number of samples included in the surface scan.

The average spacing of roughness elements is quantified by

$$
W=\frac{2 N \Delta x}{n}
$$


where $\Delta x$ is the scanning resolution, and $n$ is the number of times the roughness distribution crosses the mean surface elevation.

The effects of protrusions and depressions on the boundary layer development are expected to be different. The tendency of the roughness pattern toward protrusions or depressions is identified by the skewness of the roughness height distribution:

$$
S_{s k}=\frac{1}{k_{r m s}{ }^{3} N} \sum_{i=1}^{N}\left(y_{i}-\mu\right)^{3}
$$

For positive values of $S_{s k}$, the peaks of the roughness elements lie on average further from the mean elevation compared to the depths of the indentations, and negative values indicate the inverse.

The chosen range of roughness conditions are consistent with the roughness patterns typically observed on low pressure turbine blades of in-service gas turbine engines. This is based on comparison of the values of $k_{r m s}$ documented by Bons et al. (2001) ranging between $2.4 \mu \mathrm{m}$ and $8.8 \mu \mathrm{m}$ for the mid-span/mid-chord region of turbine blades, and normalizing these values by typical blade chords $(c)$ for lowpressure turbines between 5 and $15 \mathrm{~cm}$. Because the Reynolds numbers and pressure distributions of the current study are representative of those of low-pressure turbines, the values of $\theta / L$ of the current study will be representative of values of $\theta / c$ in low pressure turbines. Thus, comparison of the roughness height based on $k_{r m s} / L$ or $k_{r m s} / c$ is justified. The range of $k_{r m s} / c$ between $16 \times 10^{-6}$ and $175 \times 10^{-6}$ based on 
the measurements of Bons et al. (2001) compares favourably to the present values of $k_{r m s} / L$ ranging from $25 \times 10^{-6}$ to $150 \times 10^{-6}$. It is noted that the blade chord, or length of the test surface, are not of direct relevance to roughness effects. However, the similarity of Reynolds numbers and pressure distributions of the present study to those found in low-pressure turbines justifies comparison of the roughness levels based on $k_{r m s} / c$ or $k_{r m s} / L$. The corresponding range of skewness measured by Bons et al. (2001) is $-0.11<S_{s k}<+0.72$ and the range of spacing is estimated from their data to be $40<W / k_{r m s}<150$. These also compare reasonably well with the ranges of the present study of $-0.6<S_{s k}<+0.4$, and $9<W / k_{r m s}<90$.

\subsection{Test Matrix}

As noted earlier, this portion of the study includes measurements for seven surfaces of different roughness (including a smooth surface), three Reynolds numbers, three turbulence levels, and two different pressure distributions. Since not all of the surfaces were tested for each of these turbulence, Reynolds number, and pressure distribution settings, the test matrix for the current portion of this study is summarized in Table 6.2, and described in the following section.

The surfaces with $k_{r m s}$ values of $0.7 \mu \mathrm{m}, 31 \mu \mathrm{m}, 53 \mu \mathrm{m}, 107 \mu \mathrm{m}$, and $185 \mu$ mwere tested for three levels of free-stream turbulence intensity, which were about $0.5,2.5$, and $4.5 \%$. This range of surface conditions was deemed adequate for establishing the sensitivity of roughness effects to free-stream turbulence. The remaining two surfaces $\left(k_{r m s}=33 \mu \mathrm{m}\right.$, and $\left.k_{r m s}=48 \mu \mathrm{m}\right)$ were tested only for the 
Table 6.2: Test matrix for rough surface measurements

\begin{tabular}{|c|c|c|c|}
\hline $\begin{array}{c}k_{r m s} \\
(\mu \mathrm{m})\end{array}$ & $\begin{array}{c}\text { Range of } \\
R e_{L}\left(\times 10^{3}\right)\end{array}$ & $\begin{array}{c}\text { Range of } \\
\text { Tu }(\%)\end{array}$ & $\begin{array}{c}\text { Range of } \\
C_{P}\end{array}$ \\
\hline 0.7 & $145,350,470$ & $0.5,2.5,4.5$ & $C_{P 1}, C_{P 4}$ \\
\hline 31 & 350,470 & $0.5,2.5,4.5$ & $C_{P 1}, C_{P 4}$ \\
\hline 33 & 350,470 & 0.5 & $C_{P 1}$ \\
\hline 48 & 350,470 & 0.5 & $C_{P 1}$ \\
\hline 53 & 350,470 & $0.5,2.5,4.5$ & $C_{P 1}$ \\
\hline 107 & 350,470 & $0.5,2.5,4.5$ & $C_{P 1}$ \\
\hline 185 & $145,350,470$ & $0.5,2.5,4.5$ & $C_{P 1}, C_{P 4}$ \\
\hline
\end{tabular}

low free-stream turbulence conditions $\left(T u_{\text {ref }} \approx 0.5 \%\right.$ ), and are primarily intended for establishing any possible effects of roughness geometry.

All surfaces were tested for two values of flow Reynolds number, which were 350,000 and 470,000, based on the plate length of $L=1220 \mathrm{~mm}$, and reference velocity values of about 4.5 and $6.0 \mathrm{~m} / \mathrm{s}$ (measured at $x=25 \mathrm{~mm}, y=25 \mathrm{~mm}$ ). For the $k_{r m s}=0.7 \mu \mathrm{m}$ and $185 \mu \mathrm{m}$ surfaces, measurements were also obtained at a flow Reynolds number of $145,000\left(U_{\text {ref }}=1.9 \mathrm{~m} / \mathrm{s}\right)$, for the $C_{P 4}$ pressure distribution.

Streamwise distributions of the acceleration parameter $\left(\eta=\left(\nu / U_{e}^{2}\right) d U e / d x\right)$ are shown in Fig. 6.1. These results are for the smooth-surface test cases at $R e_{L}=350,000$, and are similar for the remainder of the test cases. All of the surfaces were tested with the $C_{P 1}$ pressure distribution. The distribution designated as $C_{P 4}$ has an initial acceleration similar to the $C_{P 1}$ distribution, which is followed by a milder adverse pressure gradient. The milder adverse pressure gradient results in a considerably smaller separation bubble, as seen in Fig. 6.2. Measurements with the $C_{P 4}$ pressure distribution were limited to the $k_{r m s}=0.7 \mu \mathrm{m}, 31 \mu \mathrm{m}$, and $185 \mu \mathrm{m}$ surfaces. 


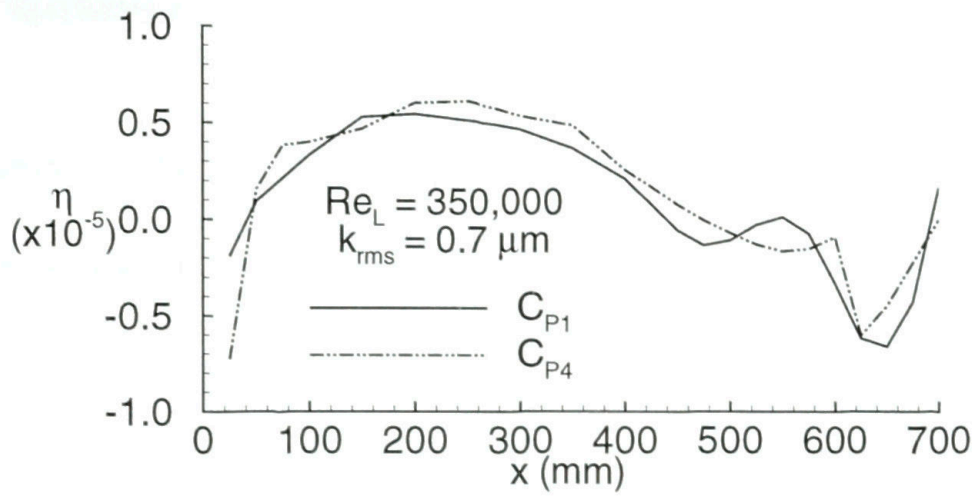

Figure 6.1: Streamwise distribution of the acceleration parameter
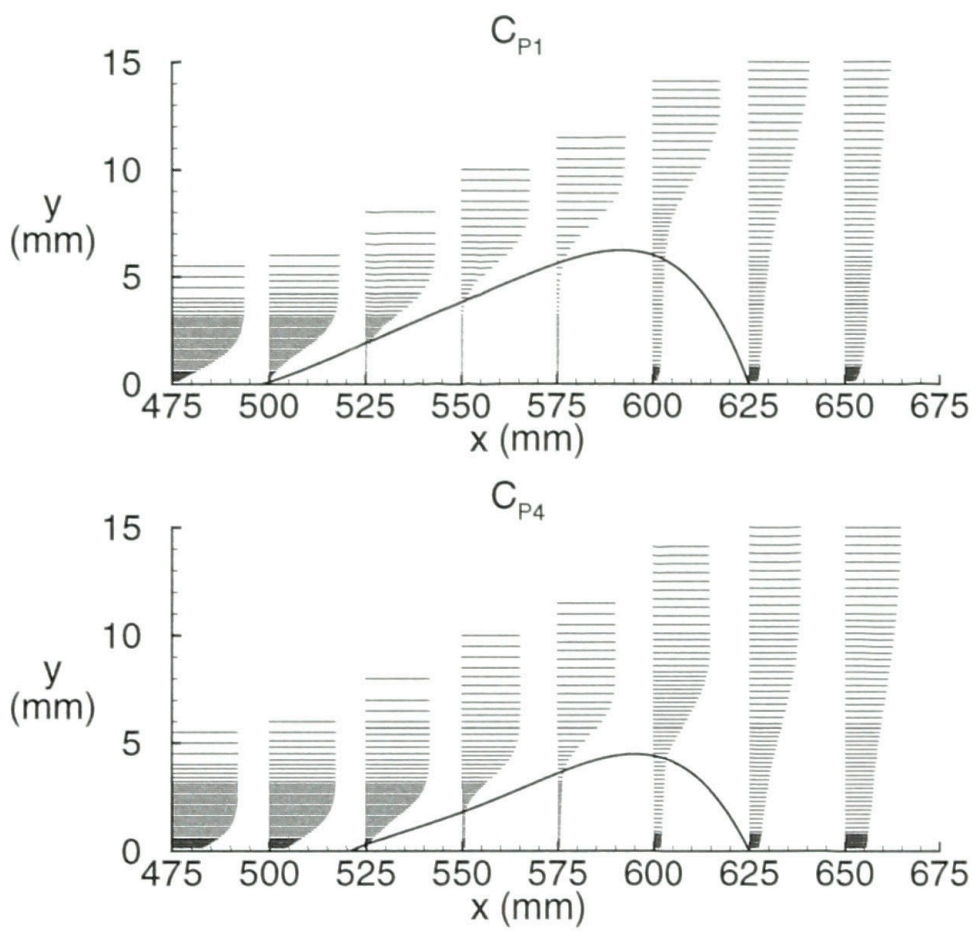

Figure 6.2: Variation of the time-averaged streamwise velocity field with pressure distribution - the separation-bubble contour is shown with the dividing streamline. 


\subsection{Results and Discussion}

\subsubsection{Effects of Surface Roughness Height and Free-Stream Turbulence}

For reference, the flow and surface parameters relevant to the discussions of this section are presented in Tables 6.3 and 6.4. For clarity, the results for two of the surfaces $\left(k_{r m s}=33 \mu \mathrm{m}\right.$ and $\left.48 \mu \mathrm{m}\right)$ and those for the $C_{P 4}$ pressure distribution, are omitted from these tables. These cases are discussed separately in Sections 6.3.3, in the context of the effects of roughness geometry and pressure gradient.

Streamwise distributions of boundary layer displacement thickness $\left(\delta^{*}\right)$ and shape factor $(H)$ are presented in Figures 6.3 and 6.4, respectively, for the surfaces for which measurements at elevated free-stream turbulence levels were obtained $\left(k_{r m s}=0.7 \mu \mathrm{m}, 31 \mu \mathrm{m}, 53 \mu \mathrm{m}, 107 \mu \mathrm{m}\right.$, and $\left.185 \mu \mathrm{m}\right)$. In these, and all other rough surface measurements, an effective location for $y=0$ needs to be established for use in the computation of $\delta^{*}$ and $H$. For the present study, $y=0$ is taken as the mean surface elevation, $\mu$ (Eq. 6.2), averaged over the test surface area. This choice is based on the argument that flow regions below this location are of very low momentum, and are unlikely to participate significantly in the dynamics of the boundary layer. The uncertainty in the position of the hot-wire sensor at the beginning of each boundary layer traverse with respect to the peaks of the roughness elements is $\pm 0.15 \mathrm{~mm}$. The resulting uncertainty in the displacement thickness is thus conservatively estimated to be $\pm 0.15 \mathrm{~mm}$. Uncertainty in probe positioning has a lesser effect on the calculation of momentum thickness $( \pm 0.05 \mathrm{~mm})$ than on $\delta^{*}$. With the present values of $\delta^{*}$ and 


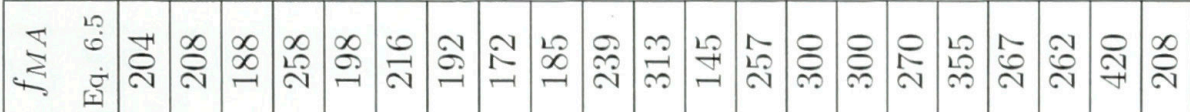

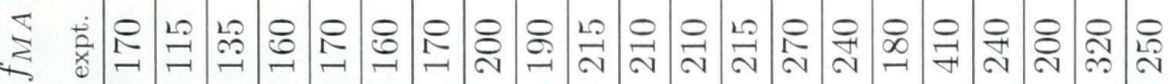

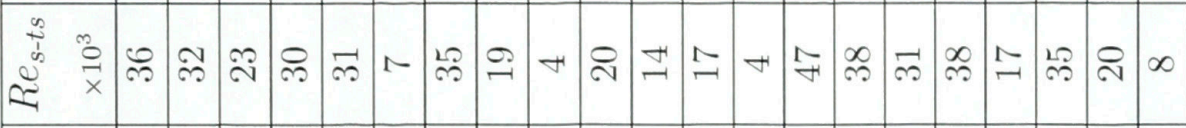

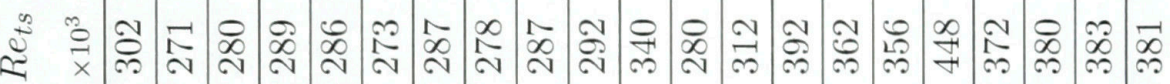
巳

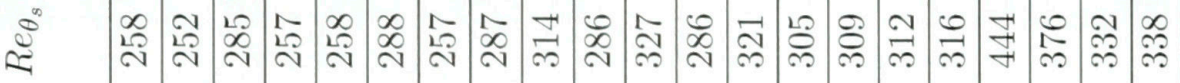

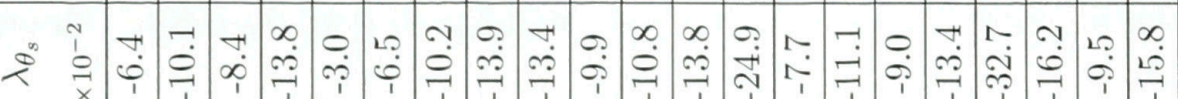

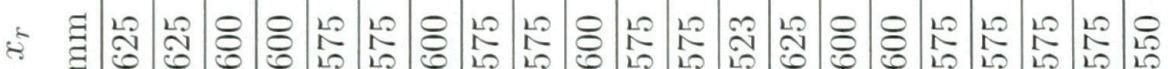
- lo la la $l 2$ la

䑻

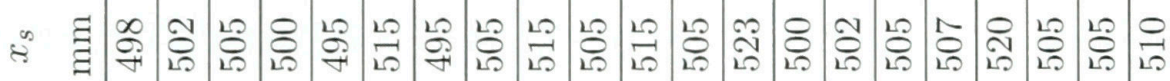

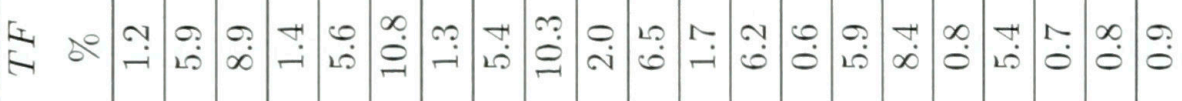

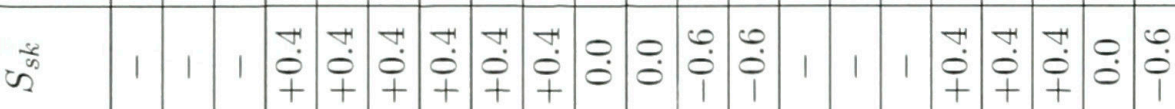

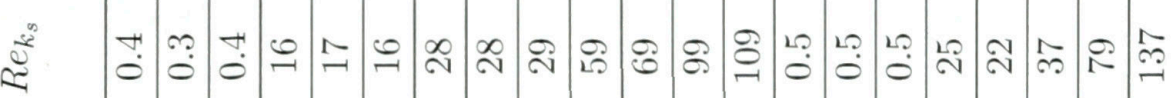

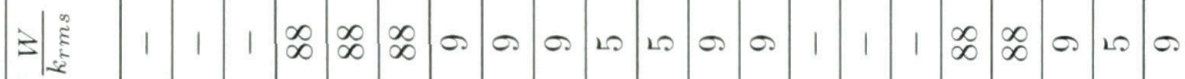

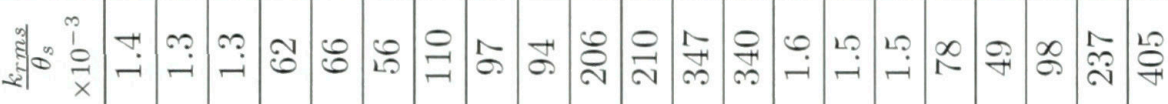
点

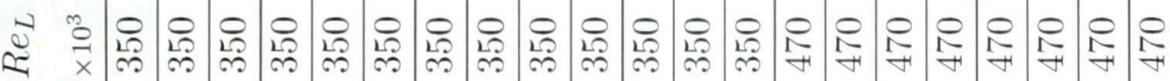


Table 6.4: Rough surface attached flow transition data ( $C_{P 1}$ pressure distribution)

\begin{tabular}{|c|c|c|c|c|c|c|c|c|c|c|c|c|}
\hline $\begin{array}{c}R e_{L} \\
\times 10^{3}\end{array}$ & $\begin{array}{c}k_{r m s} \\
\mu \mathrm{m}\end{array}$ & $\begin{array}{c}\frac{k_{r m s}}{\theta_{s}} \times 10^{-3} \\
k_{r m s}\end{array}$ & $\frac{R e_{k_{t s}}}{k_{r m}}$ & $\begin{array}{c}T u_{r e f} \\
\%\end{array}$ & $\begin{array}{c}T F \\
\%\end{array}$ & $\begin{array}{c}\lambda_{\theta_{t s}} \\
\times 10^{-2}\end{array}$ & $R e_{\theta_{t s}}$ & $\begin{array}{c}R e_{t s} \\
\times 10^{3}\end{array}$ & $\begin{array}{c}f_{M A} \\
\text { expt. }\end{array}$ & $\begin{array}{c}f_{M A} \\
\text { Eq. } \\
6.5\end{array}$ \\
\hline 350 & 107 & 188 & 5 & 57 & 0.0 & 4.8 & 11.9 & -26.8 & 304 & 279 & 350 & 311 \\
\hline 350 & 185 & 347 & 9 & 109 & -0.6 & 4.5 & 11.2 & -12.2 & 312 & 293 & 270 & 265 \\
\hline 470 & 31 & 89 & 88 & 18 & +0.4 & 4.1 & 10.3 & -3.1 & 245 & 317 & 255 & 262 \\
\hline 470 & 53 & 131 & 9 & 37 & +0.4 & 2.4 & 5.3 & 5.2 & 278 & 310 & 360 & 241 \\
\hline 470 & 53 & 141 & 9 & 33 & +0.4 & 4.1 & 10.2 & -1.7 & 286 & 342 & 270 & 245 \\
\hline 470 & 107 & 209 & 5 & 90 & 0.0 & 2.6 & 6.2 & -24.8 & 430 & 442 & 230 & 405 \\
\hline 470 & 107 & 235 & 5 & 79 & 0.0 & 4.8 & 11.9 & -26.0 & 336 & 375 & 385 & 414 \\
\hline 470 & 185 & 411 & 9 & 145 & -0.6 & 2.5 & 5.8 & -20.5 & 354 & 397 & 300 & 325 \\
\hline 470 & 185 & 568 & 9 & 136 & -0.6 & 4.5 & 11.2 & -8.1 & 266 & 368 & 385 & 365 \\
\hline
\end{tabular}

$\theta$ in the laminar boundary layer of approximately $0.5 \mathrm{~mm}$ and $0.25 \mathrm{~mm}$ respectively, the resulting uncertainty in the shape factor is \pm 0.2 . As the boundary layer thickness increases, the relative uncertainties in $\delta^{*}$ and $\theta$ are diminished. The shape factors at the location of separation, and in the turbulent boundary layer, are considered accurate within \pm 0.1 and \pm 0.05 , respectively.

In the laminar boundary layer, up to approximately $x=500 \mathrm{~mm}$, there is little effect of flow Reynolds number and surface roughness on the distributions of $\delta^{*}$ and $H$, within the noted uncertainties of $\delta^{*}$ and $H$. This suggests that the development of the laminar boundary layer prior to transition is largely unaffected by these parameters, at least within the ranges considered in this study. Thus, the variations in the transition onset and completion locations that will be discussed shortly are the result of changes in the transition process as the Reynolds number, surface roughness and turbulence intensity are varied.

The rapid growth and subsequent decrease in $\delta^{*}$ and $H$ seen between about 


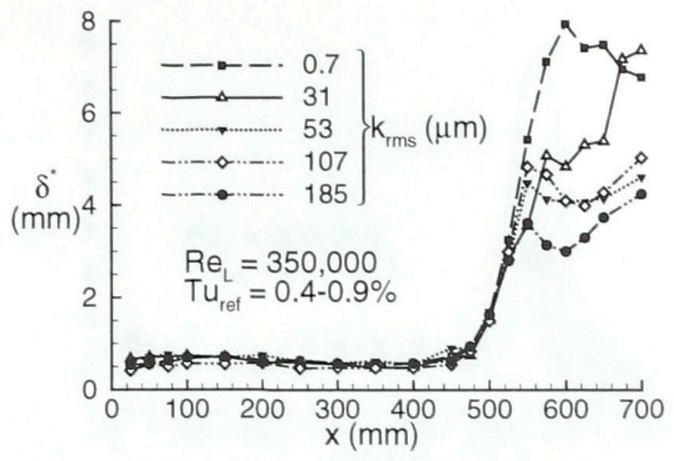

(a)

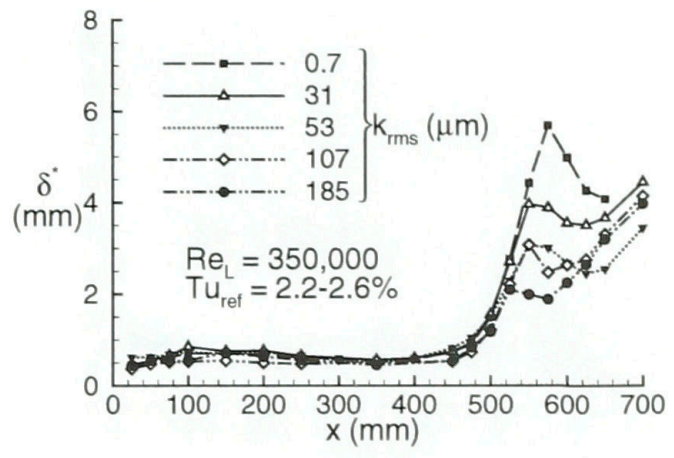

(b)

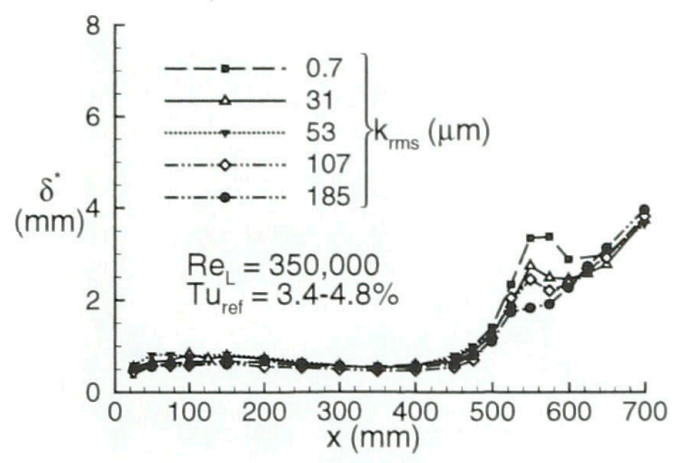

(c)

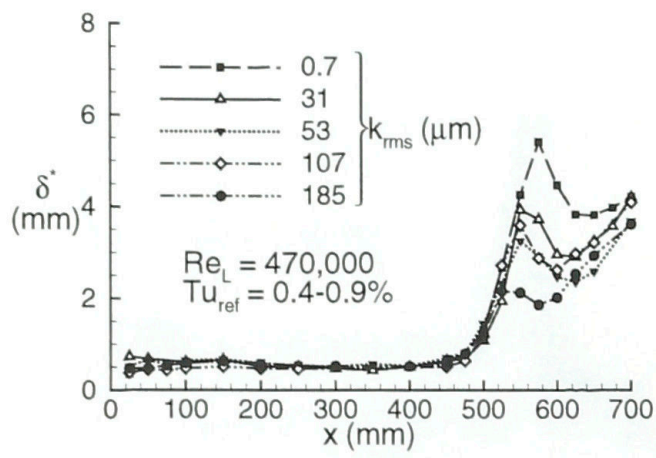

(d)

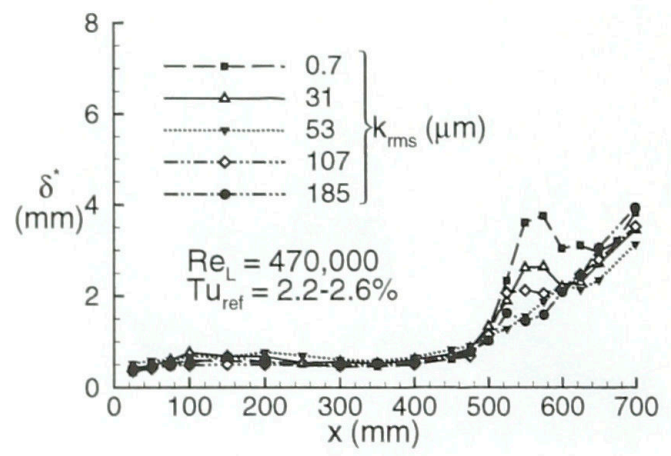

(e)

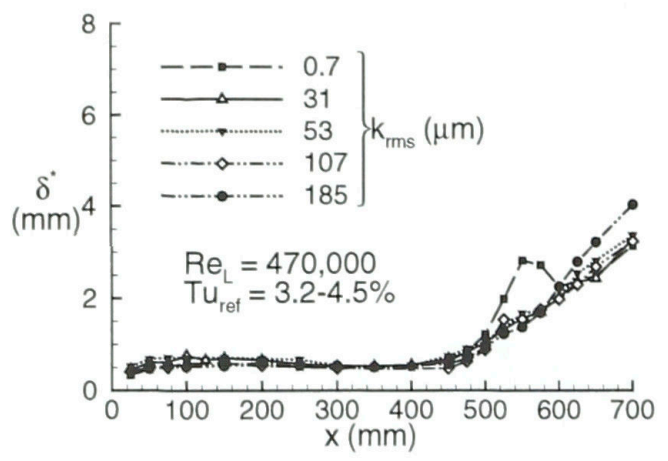

(f)

Figure 6.3: Distributions of boundary layer displacement thickness
(a)-(c): $R e_{L}=350,000$;
$(\mathrm{d})-(\mathrm{f}): R e_{L}=470,000$ 


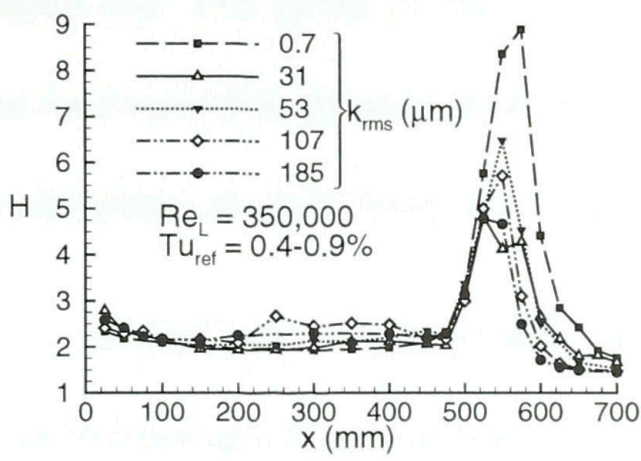

(a)

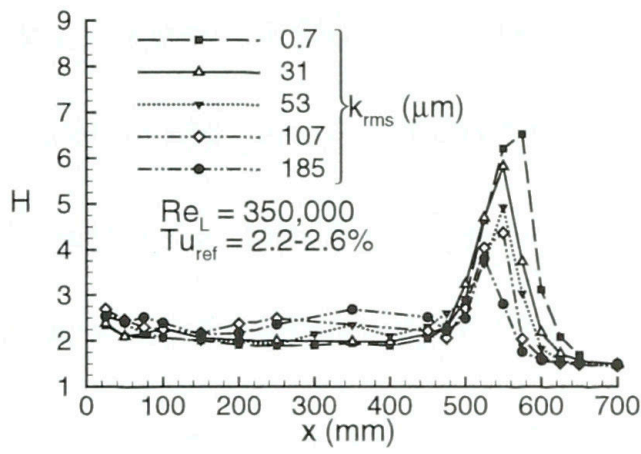

(b)

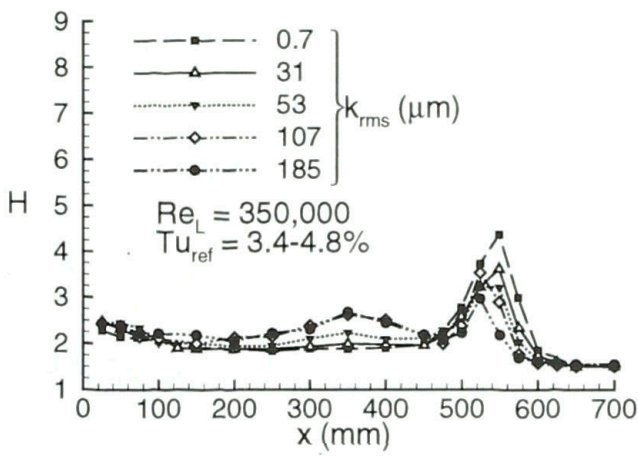

(c)

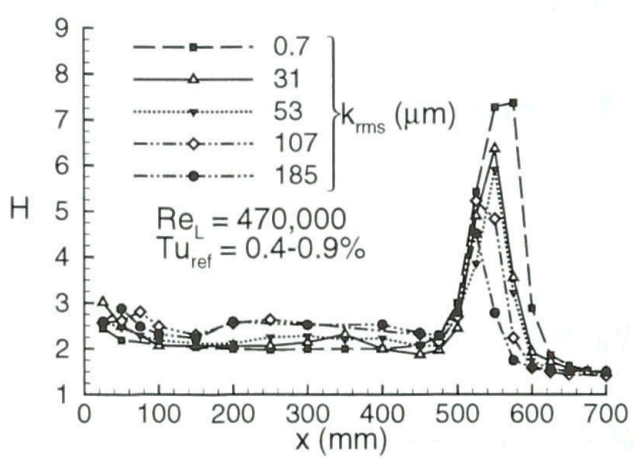

(d)

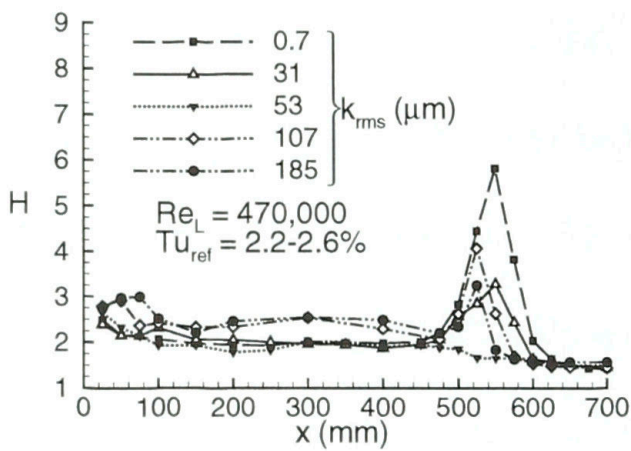

(e)

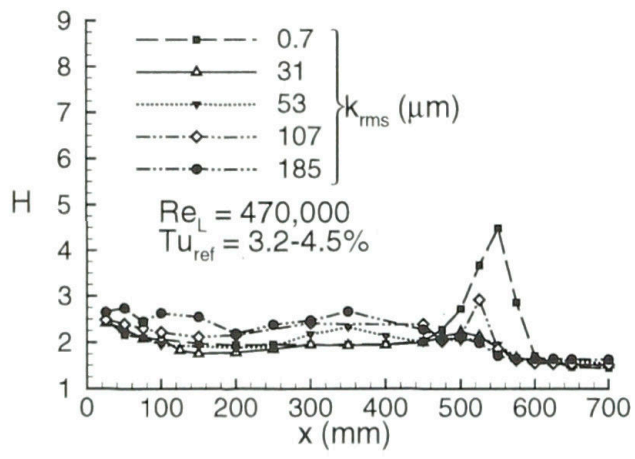

(f)

Figure 6.4: Distributions of boundary layer shape factor (a)-(c): $R e_{L}=350,000 ;(\mathrm{d})-(\mathrm{f}): R e_{L}=470,000$ 
$x=500 \mathrm{~mm}$ and $x=600 \mathrm{~mm}$ in many of the test cases is indicative of the presence of a separation bubble. This trend is most prominent in the distributions of $H$ in Figure 6.4. The peaks in these integral parameters near $x=550 \mathrm{~mm}$ coincide with the locations of maximum separation bubble thickness, with the magnitudes of these peaks giving an indication of the relative thickness of the bubble.

Examination of the velocity profiles reveals a reduction in the bubble thickness with increasing surface roughness. This trend is reflected in the peak values of the integral parameters in Figs. 6.3 and 6.4. In the case of the lowest turbulence level $\left(T u_{r e f}=0.4-0.9 \%\right)$, the effect of an increase in the RMS roughness height from $0.7 \mu \mathrm{m}$ to $185 \mu \mathrm{m}$ or roughly $20 \%$ of the boundary layer displacement thickness at separation, on the size of the separation bubble is seen to be similar to that of an increase in free-stream turbulence intensity from about $0.5 \%$ to $4.8 \%$ over the smooth surface. Thus, it may be concluded that surface roughness and free-stream turbulence are equally important to the transition process for ranges of these parameters typical of low-pressure turbine blades.

The trends for $T u_{r e f}=2.2-2.6 \%$ in Figures 6.3-e and 6.4-e suggest the presence of a small separation bubble for $k_{r m s}=185 \mu \mathrm{m}$ and $k_{r m s}=107 \mu \mathrm{m}$ but not for $k_{r m s}=53 \mu \mathrm{m}$. The average spacing of the roughness elements are very similar for the surfaces with $k_{r m s}=107 \mu \mathrm{m}(W=540 \mu \mathrm{m})$ and $k_{r m s}=53 \mu \mathrm{m}$ ( $W=490 \mu \mathrm{m}$ ), while there is a bias towards protrusions for the latter surface as is evident from the value of the skewness parameter $\left(S_{s k}=+0.42\right.$ for $k_{r m s}=53 \mu \mathrm{m}$, compared to -0.05 for $\left.k_{r m s}=107 \mu \mathrm{m}\right)$. These results demonstrate that geometric 
features of the roughness pattern other than $k_{r m s}$ are important in quantifying the effects of roughness on transition. In particular, a bias towards protrusions combined with an RMS height of $53 \mu \mathrm{m}$ appears to have been more effective in suppressing the separation bubble than an RMS roughness height of $107 \mu \mathrm{m}$ with a very small bias towards depressions. These issues will be discussed in greater detail in Section 6.3.3.

\subsubsection{Transition Inception}

The location of transition inception, $x_{t s}$ is presented for $R e_{L}=350,000$ and $R e_{L}=470,000$ in Tables 6.5 and 6.6, respectively. In the test cases with a separation bubble, this location falls into the range where the boundary layer was traversed with high resolution in the streamwise direction, hence is resolved within $\pm 2 \mathrm{~mm}$ in most cases. In the test cases with the highest turbulence and roughness levels, distinguishing turbulent spots from velocity fluctuations caused by the free-stream turbulence and roughness elements becomes more difficult, hence the uncertainty in $x_{t s}$ is judged to be $\pm 6 \mathrm{~mm}$. In the test cases where the flow remains attached, the uncertainty in $x_{t s}$ is dictated by the streamwise spacing of the measurements ( 25 to $50 \mathrm{~mm})$.

At the lowest free-stream turbulence level, transition inception is noted to occur further upstream on each of the rough surfaces, compared to the smooth surface. However, within the uncertainty in $x_{t s}$, the transition inception locations are nearly the same for the surfaces with $k_{r m s}=31 \mu \mathrm{m}$ and $k_{r m s}=53 \mu \mathrm{m}$ at both $R e_{L}=350,000\left(x_{t s}=559 \mathrm{~mm}\right.$ for $k_{r m s}=31 \mu \mathrm{m}$ vs. $\left.561 \mathrm{~mm} k_{r m s}=53 \mu \mathrm{m}\right)$ and $R e_{L}=470,000\left(x_{t s}=555 \mathrm{~mm}\right.$ for both surfaces). This similarity in the value of 
Table 6.5: Variation in transition inception locations $(\mathrm{mm})$ with surface roughness and free-stream turbulence: $R e_{L}=350,000, C_{P 1}$ pressure distribution

\begin{tabular}{|l|c|c|c|}
\hline$k_{\text {rms }} \backslash$ Tu $u_{\text {ref }}$ & $0.4-0.9 \%$ & $2.2-2.6 \%$ & $3.4-4.8 \%$ \\
\hline $0.7 \mu \mathrm{m}$ & 567 & 569 & 537 \\
\hline $31 \mu \mathrm{m}$ & 559 & 551 & 529 \\
\hline $53 \mu \mathrm{m}$ & 561 & 541 & 523 \\
\hline $107 \mu \mathrm{m}$ & 545 & 539 & $521^{*}$ \\
\hline $185 \mu \mathrm{m}$ & 537 & 529 & $499^{*}$ \\
\hline \multicolumn{4}{|c|}{$*$ denotes test case with attached flow transition }
\end{tabular}

Table 6.6: Variation in transition inception locations $(\mathrm{mm})$ with surface roughness and free-stream turbulence: $R e_{L}=470,000, C_{P 1}$ pressure distribution

\begin{tabular}{|l|c|c|c|}
\hline$k_{\text {rms }} \backslash T u_{\text {ref }}$ & $0.4-0.9 \%$ & $2.2-2.6 \%$ & $3.4-4.8 \%$ \\
\hline $0.7 \mu \mathrm{m}$ & 567 & 558 & 539 \\
\hline $31 \mu \mathrm{m}$ & 555 & 445 & $550^{*}$ \\
\hline $53 \mu \mathrm{m}$ & 555 & $450^{*}$ & $550^{*}$ \\
\hline $107 \mu \mathrm{m}$ & 535 & $525^{*}$ & $509^{*}$ \\
\hline $185 \mu \mathrm{m}$ & 521 & $505^{*}$ & $499^{*}$ \\
\hline \multicolumn{2}{|r|}{ * denotes test case with attached flow transition } \\
\hline
\end{tabular}

* denotes test case with attached flow transition

$x_{t s}$ despite the difference of approximately $70 \%$ in the RMS roughness height again suggests that aspects of the roughness geometry other than $k_{r m s}$ also influence the transition process. The roughness geometries of the two surfaces in question differ most notably in the average spacing of the roughness elements, which are much larger for the surface with $k_{r m s}=31 \mu \mathrm{m}(W=2720 \mu \mathrm{m})$ than for the surface with $k_{r m s}=53 \mu \mathrm{m}(W=490 \mu \mathrm{m})$. It appears that the larger spacing of the roughness elements of the $k_{r m s}=31 \mu \mathrm{m}$ surface are more effective at initiating the transition process than the more densely packed elements of the $k_{r m s}=53 \mu \mathrm{m}$ surface. This is not particularly surprising, since with increased density of the roughness elements, the wake region and effective frontal area of each element are reduced. Of course, 


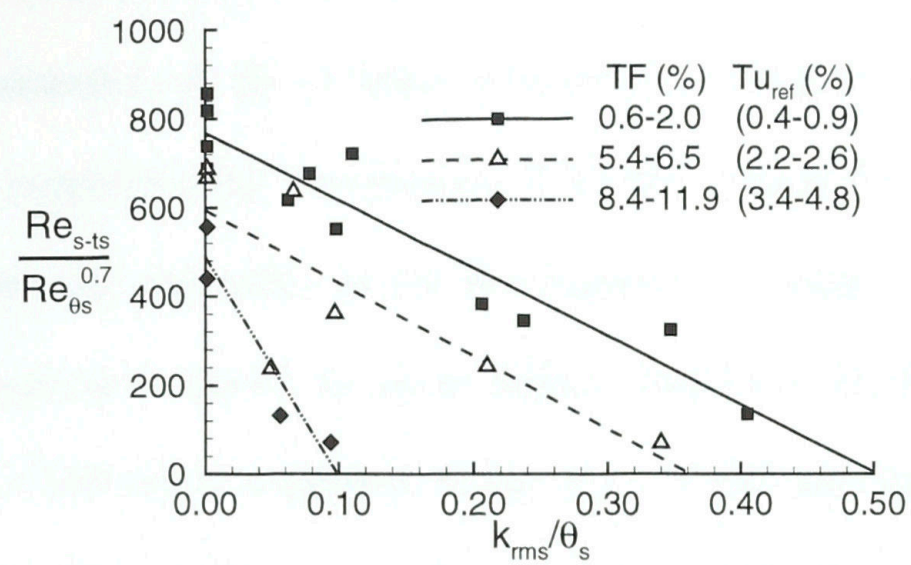

Figure 6.5: Sensitivity of the transition inception location in separation bubbles to surface roughness and free-stream turbulence

this trend would eventually reverse as the density of roughness elements is reduced further, since in the limit of both extremes a smooth surface condition would be reached.

The tabulated data also demonstrate that the transition inception location in the separation bubble remains sensitive to surface roughness as the free-stream turbulence intensity is increased. This observation is consistent for all turbulence and roughness levels tested, as illustrated in Fig. 6.5. From the trends in this figure, the sensitivity of the transition inception location to roughness height is noted to be essentially constant up to the intermediate turbulence level of $T u_{r e f}=2.2-2.6 \%$, but this sensitivity is notably increased for $T u_{r e f}=3.4-4.8 \%$. This trend is intuitive, since with increased free-stream turbulence, the transition inception location moves upstream in the separated shear layer, and therefore closer to the surface. Thus, with high free-stream turbulence, the separated shear layer is expected to respond more readily to changes in the surface conditions. 
In the attached flow transition cases, because of their limited number and the coarser resolution of the location of transition inception, the variation of $x_{t s}$ with surface roughness and free-stream turbulence cannot be identified with the same precision and generality as for the separation bubble cases. Nonetheless, transition inception is noted to occur further upstream for the $k_{r m s}=53 \mu \mathrm{m}$ surface $\left(x_{t s}=450 \mathrm{~mm}\right)$, compared to the $k_{r m s}=107 \mu \mathrm{m}$ and $k_{r m s}=185 \mu \mathrm{m}$ surfaces $\left(x_{t s}=525\right.$ and $\left.505 \mathrm{~mm}\right)$ at $T u_{r e f}=2.2-2.6 \%, R e_{L}=470,000$, and also compared to the $k_{r m s}=107 \mu \mathrm{m}$ surface at $T u_{r e f}=3.4-4.8 \%, R e_{L}=470,000$ $\left(x_{t s}=509 \mathrm{~mm}\right)$. These three surfaces all have similar values of the spacing parameter $W / k_{r m s}$. Apart from the roughness height, they differ most notably in their values of skew $\left(S_{s k}=+0.4,0.0\right.$, and -0.6 for $k_{r m s}=53 \mu \mathrm{m}, 107 \mu \mathrm{m}$, and $185 \mu \mathrm{m}$, Table 6.1). Thus, these results provide further support for the importance of the skewness of the roughness distribution on transition onset, which is discussed in the following section.

\subsubsection{Effects of Roughness Geometry}

The results presented thus far have shown the roughness height to have a significant influence on the transition process. This is the case even in separation bubbles, where the transition process is initiated away from the surface. These results also indicate there to be notable effects of other geometric properties of the surfaces, namely the spacing of the elements and skewness of the distribution. However, since the roughness heights of these surfaces are all significantly different, direct comparison between surfaces for the purpose of quantifying these effects is not 
possible. To clarify the importance of these effects, two additional rough surfaces were tested, having similar roughness heights as two of the previously tested surfaces, but with significantly different roughness geometry. The experimental results for these additional surfaces are presented in Table 6.7 and 6.8 for the $C_{P 1}$ and $C_{P 4}$ pressure distributions, respectively. For completeness, the results for the remaining test cases at the lowest turbulence level from Table 6.3 are repeated in Table 6.7.

\subsubsection{Effects of Roughness Element Spacing}

In the case of very close spacing of the roughness elements, the regions between the elements are unlikely to exchange significant mass and momentum with the exterior flow passing over the crests of the roughness elements. This situation of limited disturbance would also be present in the case of very large spacing owing to the relatively small number of roughness elements. Thus, there ought to exist an element spacing between these two extremes for which the extent of disturbances experienced by the boundary layer is maximized.

Two of the test surfaces listed in Table 6.7 with similar values of roughness height and skewness $\left(k_{r m s}=48 \mu \mathrm{m}, S_{s k}=+0.2\right.$ vs. $\left.k_{r m s}=53 \mu \mathrm{m}, S_{s k}=+0.4\right)$ provide an opportunity to determine the effect of changing the roughness element spacing from $W / k_{r m s}=73$ to $W / k_{r m s}=9$ on transition inception in separation bubbles. Based on the values of $x_{t s}-x_{s}$ in Table 6.7 for both flow Reynolds numbers tested, a slight upstream movement of the transition inception location with increased spacing of roughness elements is evident. This trend is also supported by the comparison of the results for the surfaces with $k_{r m s}=31 \mu \mathrm{m}\left(W / k_{r m s}=88, S_{s k}=+0.4\right)$ and 


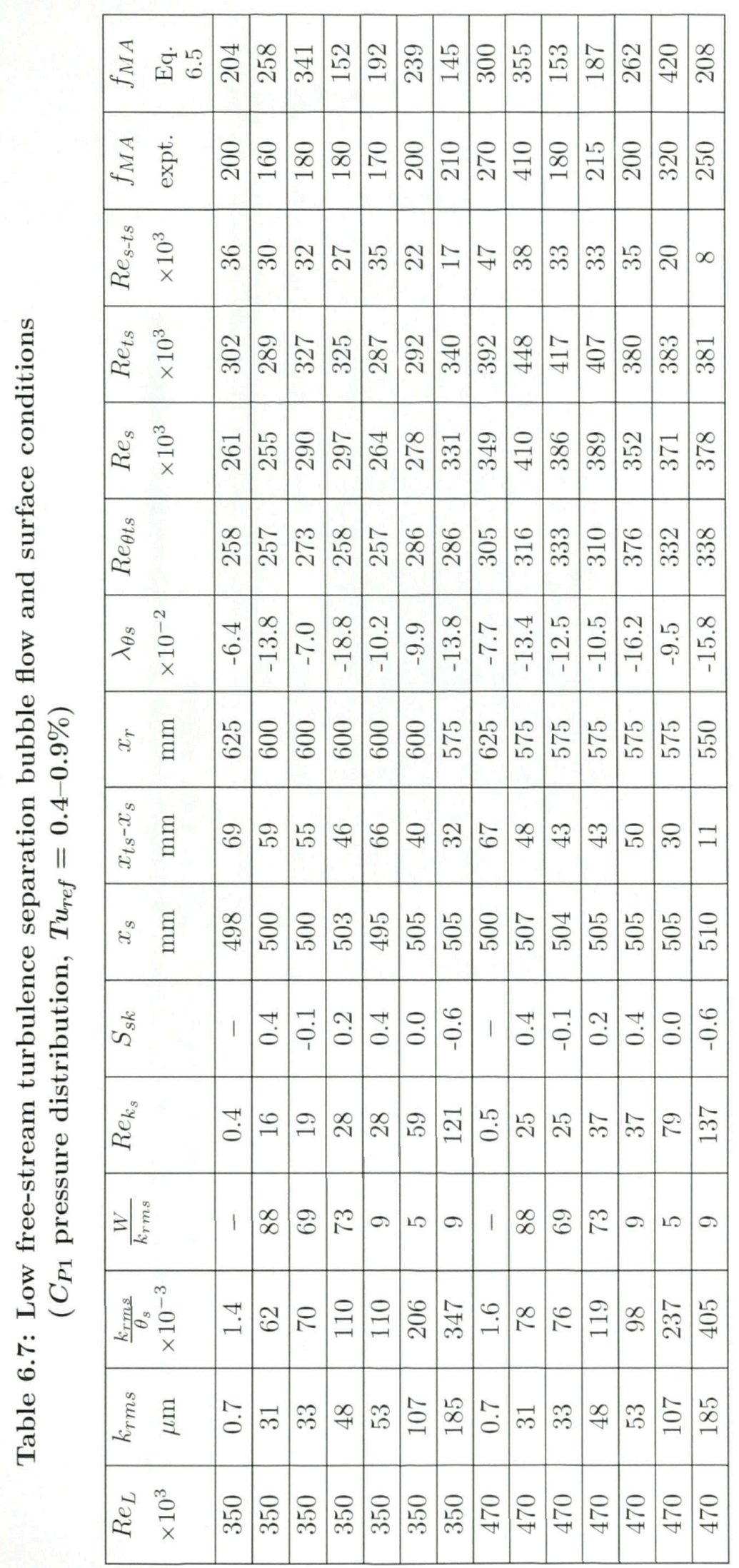




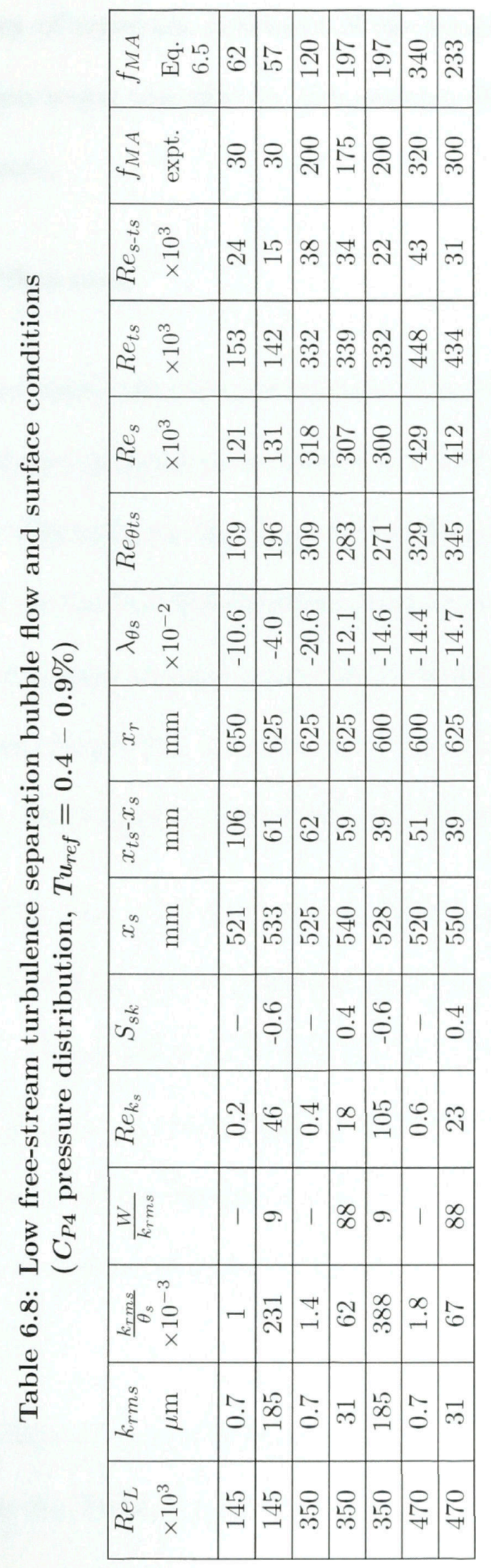


$k_{r m s}=53 \mu \mathrm{m}\left(W / k_{r m s}=9, S_{s k}=+0.4\right)$, discussed in the previous section. In these two cases, the tendency of upstream movement of the transition inception location with increased roughness height was offset by the opposing effect of increased spacing of the roughness elements.

\subsubsection{Effects of Skewness}

As described previously, the skewness represents the tendency of the roughness distribution toward sharper protrusions or depressions. Protrusions affect the local boundary layer flow by displacing the oncoming fluid and shedding vorticity, typically in an unsteady manner. In the case of depressions, disturbance of the local flow field is dependent on mass and momentum exchange between the fluid inside the depressions and the exterior boundary layer flow. In either case, the extent of the disturbance is expected to depend on the sharpness of the roughness feature.

The surfaces with $k_{r m s}=31$ and $33 \mu \mathrm{m}$ have similar roughness spacing $\left(W / k_{r m s}=88\right.$ vs. 69$)$ whereas their skewnesses are of opposite sign $\left(S_{s k}=+0.4\right.$ vs. - 0.1). For both of the tested flow Reynolds numbers, the locations of transition onset (quantified through $x_{t s}-x_{s}$ ) are slightly further upstream for the surface with negative skewness. This trend is somewhat counterintuitive, and is also inconsistent with the trend observed between the $k_{r m s}=53 \mu \mathrm{m}$ and $k_{r m s}=107 \mu \mathrm{m}$ surfaces, discussed in Section 6.3.1.

Although both the spacing and skewness of the roughness distribution are seen to have an influence on the transition location beyond the level of uncertainty in $x_{t s}$, 
these effects are secondary compared to the sensitivity of transition inception to the height of the roughness elements. For example, the effect of varying the spacing of roughness elements from $W / k_{r m s}=9$ to 88 results in considerably less movement of the transition onset location than that caused by varying $k_{r m s} / \theta_{s}$ from 0.7 to $185 \mu \mathrm{m}$. Since these ranges of $W / k_{r m s}$ and $k_{r m s} / \theta_{s}$ represent typical gas turbine roughness conditions, it is concluded that the effect of roughness on transition inception in turbomachinery blades is adequately quantified through $k_{r m s} / \theta_{s}$.

\subsubsection{Spectral Analysis}

\subsubsection{Pre-Transitional Growth of Disturbances}

Upstream movement of the transition inception location with increasing roughness height and changes in roughness geometry may be the result of changes in the frequency, initial amplitude, and/or amplification rate of disturbances at the most unstable frequencies. Spectral analysis of the present experimental results was performed to establish the relative importance of these factors.

In Fig. 6.6, Fourier power spectra of $u^{\prime} / U_{\text {ref }}$ are shown at various stages of development of the boundary layer for the $k_{r m s}=0.7 \mu \mathrm{m}$ and $k_{r m s}=107 \mu \mathrm{m}$ surfaces with the $C_{P 1}$ pressure distribution and $R e_{L}=350,000$. It is noted that the frequency at which the highest amplification rate of instability waves occurs $\left(f_{M A}\right)$ does not vary significantly between the two surface conditions. This result applies to the remainder of the tested surfaces and flow conditions as well (Tables $6.7 \& 6.8$ ). It is also noted that the measured value of $f_{M A}$ agrees closely with the prediction based on Walker's 


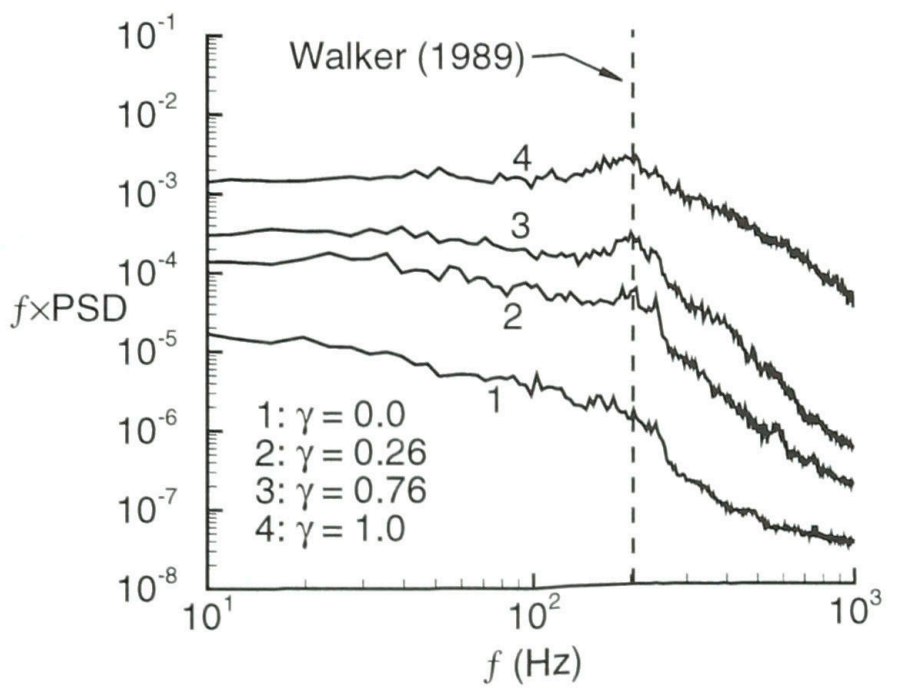

(a) $k_{r m s}=0.7 \mu \mathrm{m}$

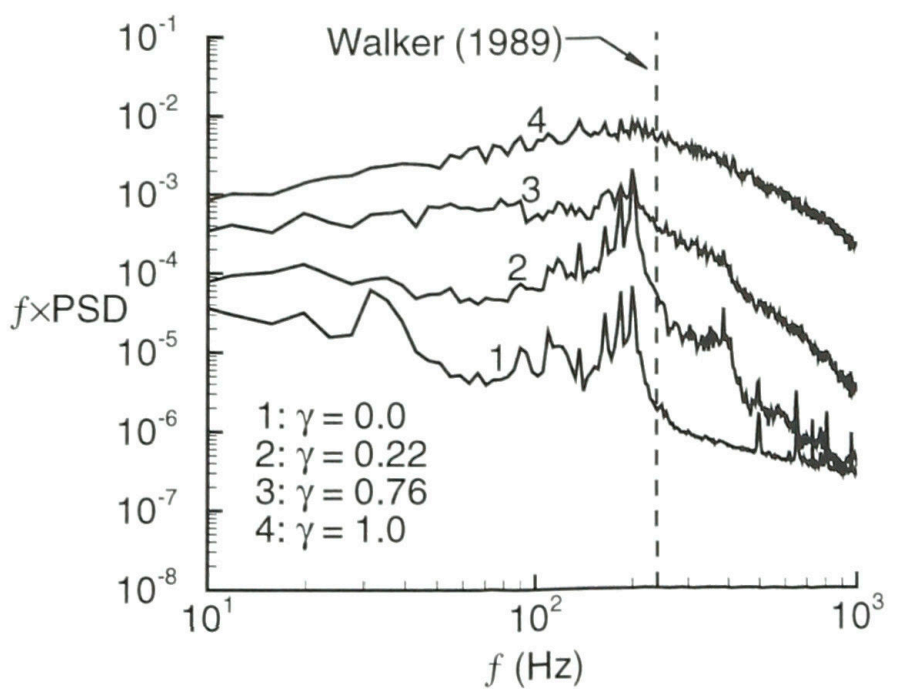

(b) $k_{r m s}=107 \mu \mathrm{m}$

Figure 6.6: Fourier power spectrum of $u^{\prime} / U_{\text {ref }}$ for $R e_{L}=350,000, C_{P 1}$ pressure distribution $(y=1.25 \mathrm{~mm})$ 
(1989) correlation developed for the growth of Tollmien Schlichting (T-S) waves in attached-flow boundary layer transition:

$$
f_{M A}=\frac{3.2 U_{e}^{2}}{2 \pi \nu R e_{\delta^{*}}^{3 / 2}}
$$

shown by a dashed vertical line in Fig. 6.6.

In cases of attached flow, the Reynolds number based on displacement thickness, $R e_{\delta^{*}}$, appearing in Eq. 6.5 is evaluated at the nearest measurement station upstream of the transition inception location. Since this expression was developed from an analysis of attached boundary layers, in cases of separation bubble transition, $R e_{\delta^{*}}$ is evaluated just prior to the separation point. The agreement between the observed peaks in the spectra and the predictions of Eq. 6.5 is similar in cases of attached flow and separation bubble transition. In the majority of experiments included in the current study (28 out of the 37 test cases), the agreement is also within the level of scatter noted by Walker while developing this correlation $( \pm 30 \%$, Tables 6.3, 6.4, 6.7, and 6.8, and summarized in Fig. 6.7). This suggests the dominant mechanism leading to transition in the majority of separation bubbles of the current study to be the growth of Tollmien-Schlichting waves. Additionally, the good agreement between the measured and predicted values of $f_{M A}$ for the various rough surfaces indicates that $f_{M A}$ is insensitive to the range of surface roughness considered in this study. 


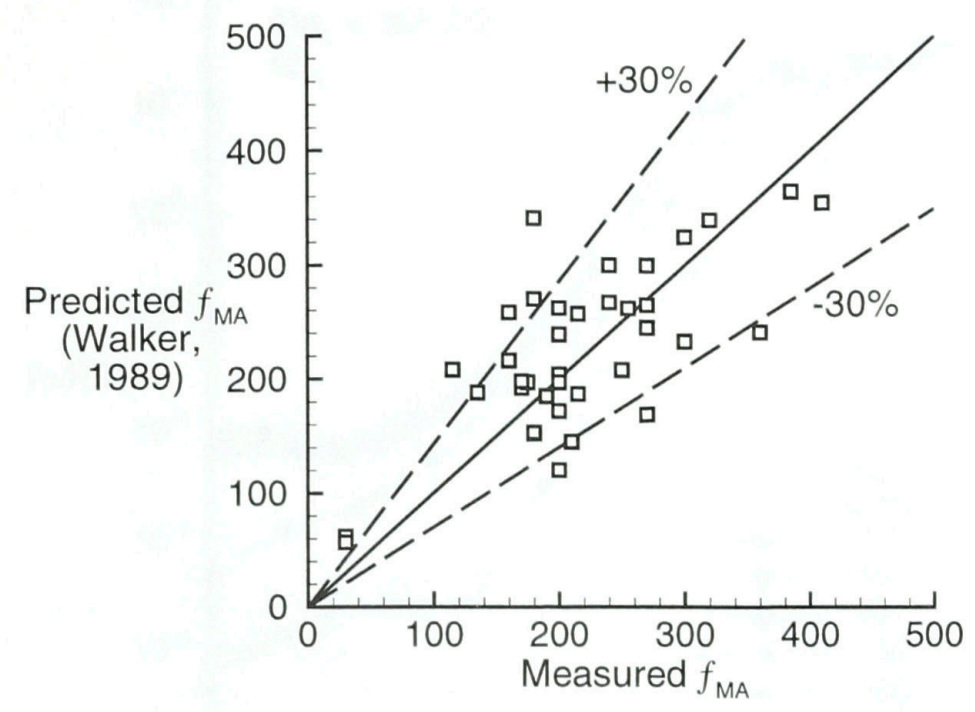

Figure 6.7: Comparison of observed dominant frequency to the correlation of Walker (1989) (Eq. 6.5)

\subsubsection{Growth Rates of Disturbances}

The streamwise growth of the energy of disturbances at the measured value of $f_{M A}$ is shown for several cases in Fig. 6.8 for $R e_{L}=350,000$. The trends in this figure were confirmed to be consistent with those for the remaining flow Reynolds numbers and surface roughness conditions. The power spectral density shown in this figure was determined by averaging the power spectrum of $u^{\prime} / U_{\text {ref }}$ within a frequency range of $\pm 15 \%$ of $f_{M A}$. Measurements for some of the surfaces are omitted from the figure for clarity. In this figure, the exponential growth rate observed shortly before transition onset is seen to be insensitive to the level of surface roughness. It is evident, however, that the initial magnitude of the disturbance at $f_{M A}$ is notably larger for the rough surfaces than for the smooth surface. This result suggests the larger initial amplitude of disturbances at $f_{M A}$ to be a significant factor in promoting earlier transition inception on rough surfaces. 


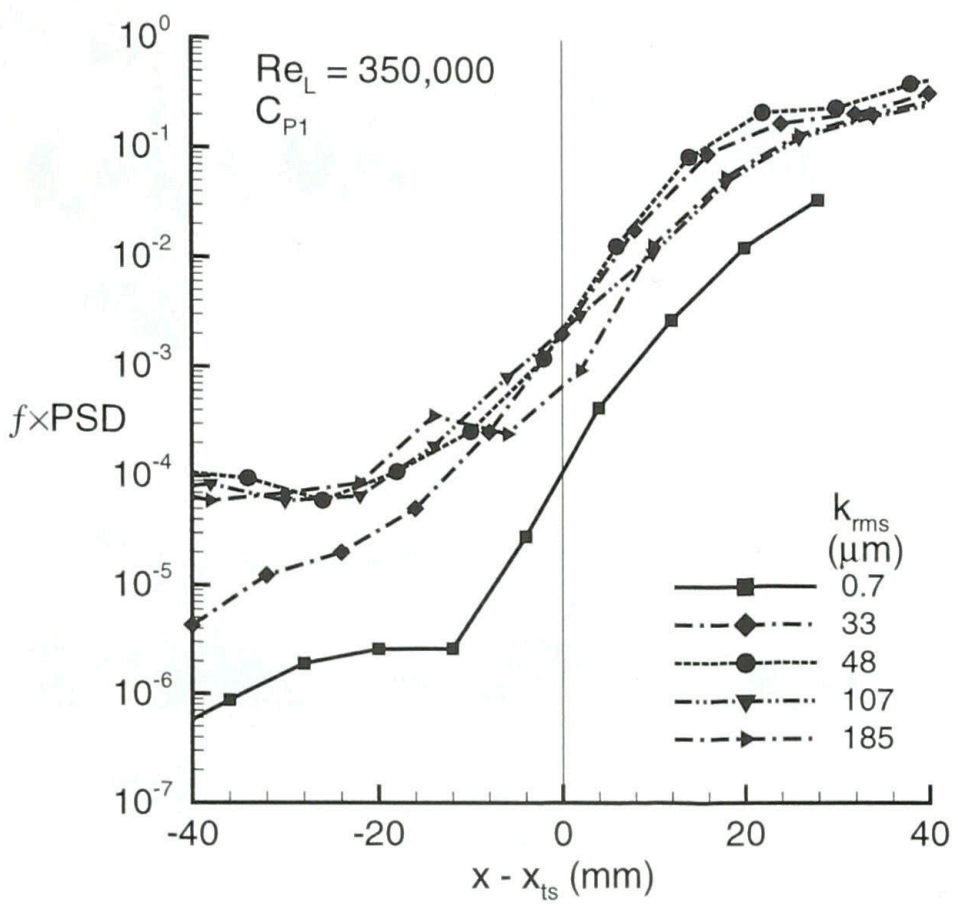

Figure 6.8: Growth rate of disturbance energy in the $u^{\prime} / U_{\text {ref }}$ spectrum at $f_{\mathrm{MA}}\left(R e_{L}=350,000, C_{P 1}\right.$ pressure distribution)

In the cases with the $C_{P 1}$ pressure distribution, the intermittent turbulence activity observed in the hot-wire velocity traces in the transition zone (Fig. 6.9-a) closely resembles the velocity traces previously observed in attached-flow transition. This, together with the presence of instability waves of dominant frequency and growth rate patterns typical of Tollmien-Schlichting waves, suggests that the transition process in these test cases is driven by viscous instability. For the test cases with the $C_{P 4}$ pressure distribution, no structures resembling turbulent spots could be identified in the hot-wire velocity traces. Instead, the transition process was characterized by large amplitude periodic velocity fluctuations at $f_{M A}$ just before transition (Fig. 6.9-b), and the breakdown to full turbulence was sudden (Fig. 6.9-c).

It is suggested that in the test cases with the $C_{P 4}$ pressure distribution viscous 


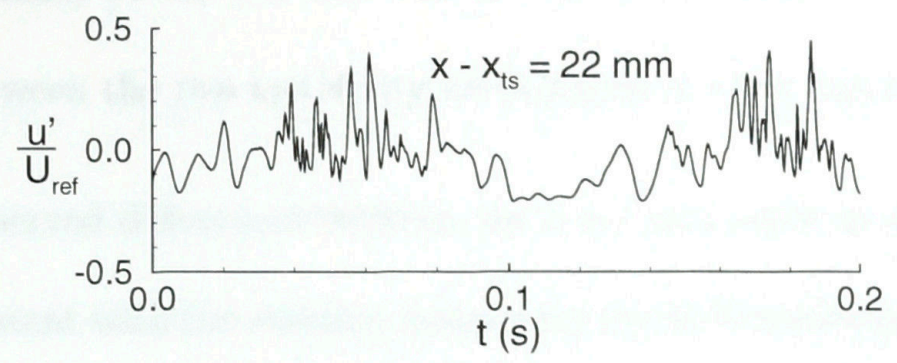

(a) $C_{P 1}$

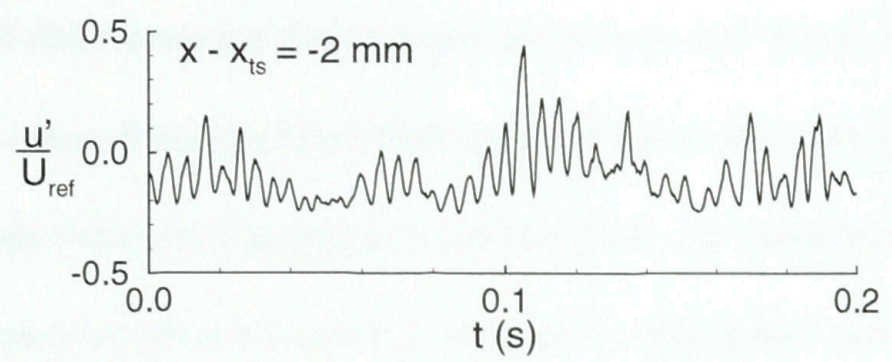

(b) $C_{P 4}$ (before transition)

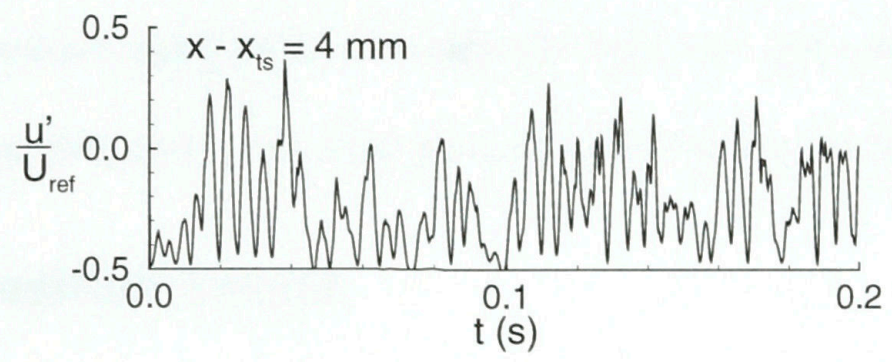

(c) $C_{P 4}$ (after transition)

Figure 6.9: Comparison of hot-wire signals in the separation bubble for the $C_{P 1}$ and $C_{P 4}$ pressure distributions

T-S instability is accompanied by the inviscid Kelvin-Helmholtz instability, and that the latter is responsible for the ultimate breakdown to turbulence. K-H instability is characterized by the formation, and often pairing, of large two-dimensional vortices in the separated shear layer (e.g. Estevadoral \& Kleis, 1999). The regular shedding of these vortical structures would likely result in large amplitude velocity fluctuations such as those seen in Fig. 6.9-b. The fact that the vorticity shedding occurs at the 
dominant frequency of the T-S waves in the separated shear layer demonstrates the interaction between the two instability mechanisms in these instances.

The observed differences between the transition patterns of the $C_{P 1}$ and $C_{P 4}$ cases are consistent with the stability bounds for the K-H mechanism. Chandrasekhar (1961) established the conditions for $\mathrm{K}-\mathrm{H}$ instability to be $0<\kappa d<1$, where $\kappa$ is the wave number of the dominant disturbance frequency, and $d$ is a shear-layer thickness parameter that arises from the hyperbolic tangent curve-fit to the local velocity profile. For the test cases with the $C_{P 4}$ pressure distribution, the parameter $\kappa d$ just upstream of transition varies between 0.4 and 0.5 , compared with values between 0.6 and 0.7 for the $C_{P 1}$ pressure distribution. The greater inviscid instability of the velocity profiles for the $C_{P 4}$ pressure distribution is consistent with the more dominant role of the $\mathrm{K}-\mathrm{H}$ mechanism observed in the transition process of these test cases.

\subsubsection{Transition Length}

Streamwise distributions of intermittency are presented for the separation bubble and attached flow test cases with the $C_{P 1}$ pressure distribution in Figures 6.10 and 6.11, respectively. For the separation bubble test cases, a notable effect of surface roughness on the transition rate is not evident in the majority of test cases, within the uncertainty of the computed intermittency distributions. This suggests that the production and spreading rates of turbulent spots are not affected by surface roughness. The lack of change in the spot propagation characteristics with surface roughness in these test cases is consistent with the observation that the portion of the free shear layer in which transition takes place is located above the peaks of the 
roughness elements.

Comparison of parts a, b and c of Figure 6.10 reveals an effect of turbulence intensity on the rate of transition in the separation bubble, with increased turbulence levels resulting in a longer transition length. This trend is consistent with the observed variations in spot production rates with free-stream turbulence in attached boundary layers (e.g. Solomon et al., 1996). The observed trend is the result of the effect of freestream turbulence on the boundary layer shape factor. As will be discussed further in Chapter 8, the transition rate correlates with the shape factor at the transition inception location $\left(H_{t s}\right)$.

A very small separation bubble appears to be present in both the $k_{r m s}=$ $107 \mu \mathrm{m}$ and $k_{r m s}=185 \mu \mathrm{m}$ test cases shown in Fig. 6.11, although these test cases have been categorized as attached flow transition in Table 6.6. It is noted that the transition rate in these two instances is similar to those of the separation bubble test cases at the same turbulence level and Reynolds number, shown in the bottom half of Fig. 6.10-b. Thus, while the separation and reattachment locations in these two cases cannot be reliably identified with the present spatial resolution of the measurements, clearly the presence of even a very short and shallow separated region is able to affect the transition rate. The different transition rates for the test cases shown in Fig. 6.11a are consistent with the variations in shape factor at transition inception between these three test cases $\left(H_{t s}=1.9,4.0\right.$, and 2.5 for the $k_{r m s}=53,107$, and $185 \mu \mathrm{m}$ surfaces in Fig. 6.11-a, respectively).

For the attached flow transition cases in Fig. 6.11, the rates of transition are 


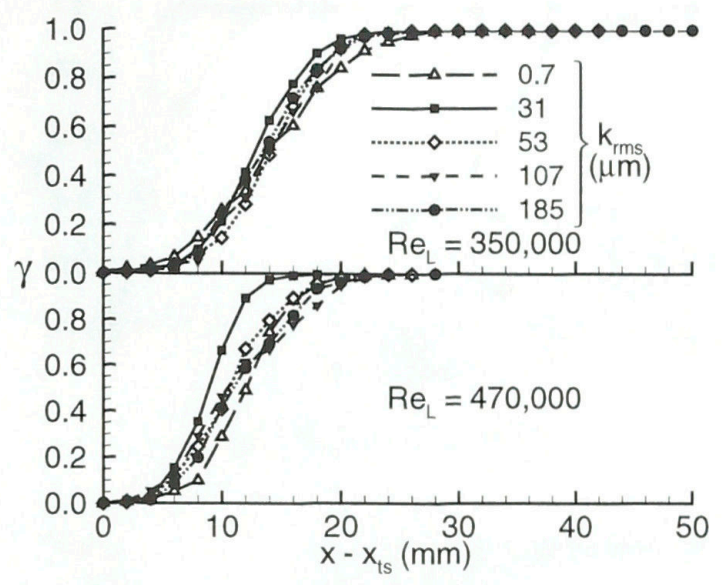

(a) $T u_{\text {ref }}=0.4-0.9 \%$

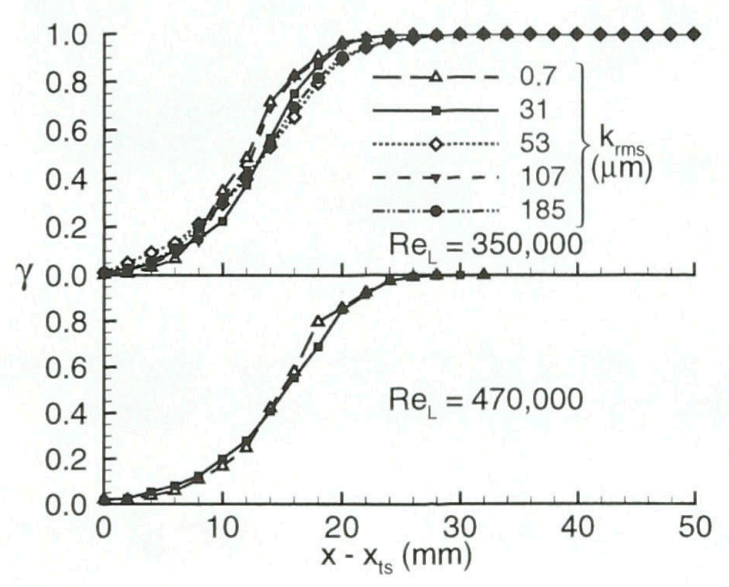

(b) $T u_{\text {ref }}=2.2-2.6 \%$

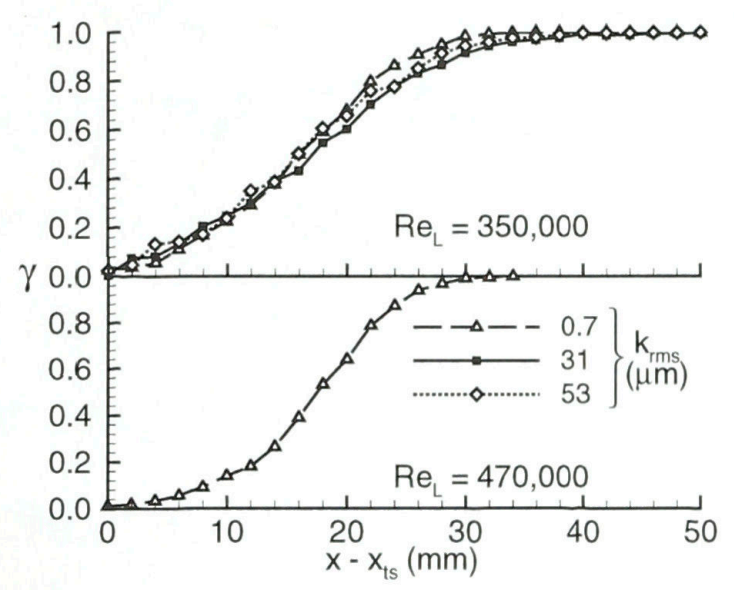

(c) $T u_{\text {ref }}=3.4-4.8 \%$

Figure 6.10: Streamwise distributions of intermittency separation bubble cases 


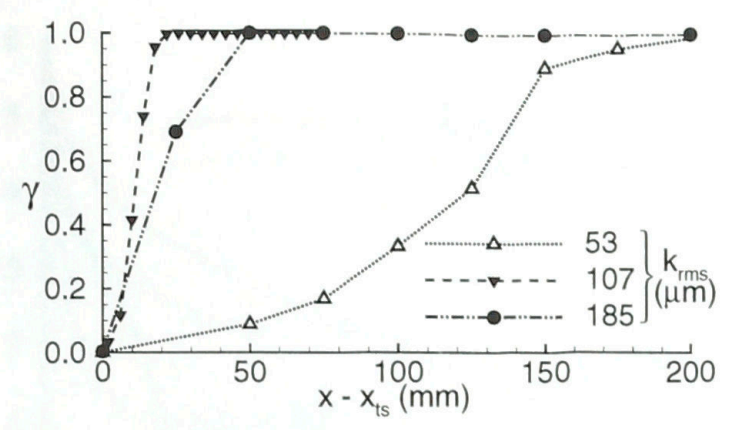

(a) $T u_{\text {ref }}=2.2-2.6 \%$

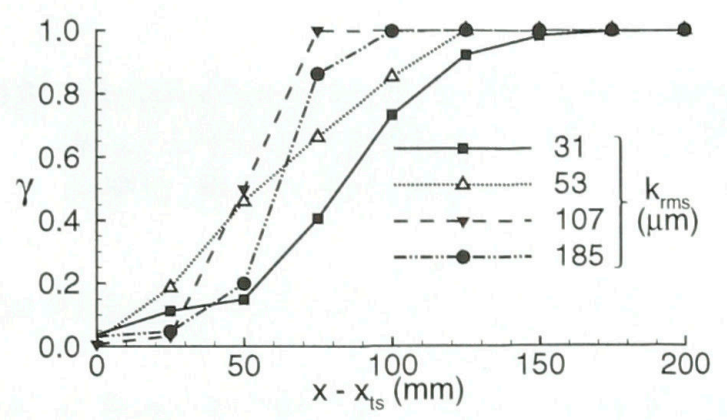

(b) $T u_{\text {ref }}=3.4-4.8 \%$

\section{Figure 6.11: Streamwise distributions of intermittency - attached flow cases $\left(R e_{L}=470,000\right)$}

observed to be lower for the $k_{r m s}=31 \mu \mathrm{m}$ and $k_{r m s}=53 \mu \mathrm{m}$ surfaces than for the surfaces with $k_{r m s}=107 \mu \mathrm{m}$ and $k_{r m s}=185 \mu \mathrm{m}$. At the lower turbulence intensity $\left(T u_{r e f}=2.2-2.6 \%\right.$ in Fig. $\left.6.11-a\right)$, this is at least partly due to differences in $H_{t s}$, since the locations of transition inception are different for these surfaces. However, the values of $H_{t s}$ are similar at $T u_{r e f}=3.4-4.8 \%$ for the $k_{r m s}=31$ $\mu \mathrm{m}, k_{r m s}=53 \mu \mathrm{m}$, and $k_{r m s}=185 \mu \mathrm{m}$ surfaces in Figure 6.11-b $\left(H_{t s}=2.0,2.0\right.$, and 2.1, respectively). Thus, an increase in RMS roughness height from $53 \mu \mathrm{m}$ to $185 \mu \mathrm{m}$ appears to affect the rate of spot production, the spot spreading rate, or both. Based on comparisons to the measurements of Chong \& Zhong (2003), the roughness elements of the surface with $k_{r m s}=185 \mu \mathrm{m}$ are expected to protrude 


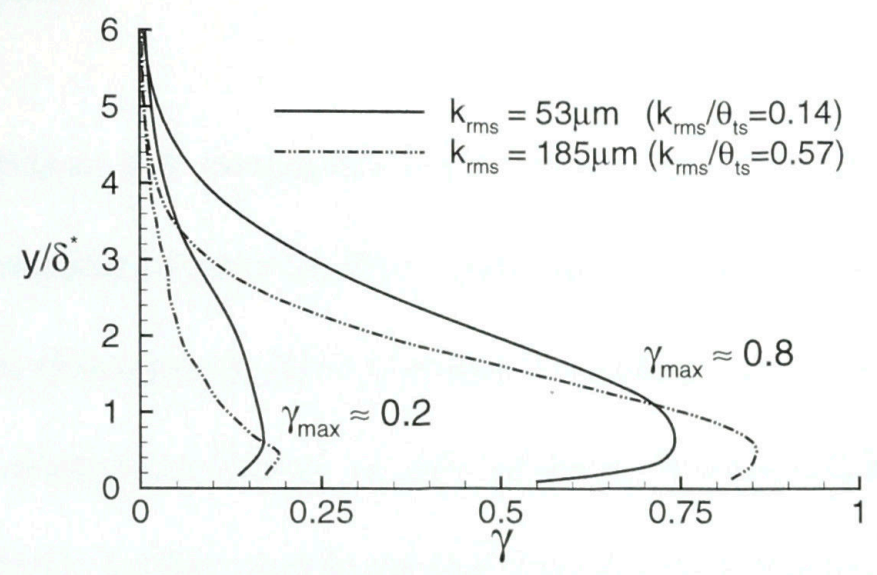

Figure 6.12: Cross-stream distributions of intermittency for $k_{r m s}=53 \mu \mathrm{m}$ and $k_{r m s}=185 \mu \mathrm{m}\left(T u_{r e f}=3.4-\right.$ $\left.4.8 \% ; R e_{L}=470,000\right)$

into the regions of the boundary layer where the turbulent spots are most active in turbulence production, at least during the earlier phases of the transition process. It is therefore plausible that the roughness elements affect the rate of production or the structure of turbulent spots. This hypothesis is supported by the visible differences in the cross-stream intermittency distributions between test cases. This is shown in Fig. 6.12 for the $k_{r m s}=53 \mu \mathrm{m}$ and $k_{r m s}=185 \mu \mathrm{m}$ surfaces at two stages in the transition process where the peak intermittencies were similar for the two surfaces $\left(\gamma_{\max } \approx 0.2\right.$, and $\left.\gamma_{\max } \approx 0.8\right)$. The more concentrated cross-stream intermittency profile for the $k_{r m s}=185 \mu \mathrm{m}$ surface, particularly at the earlier stage of transition $\left(\gamma_{\max } \approx 0.2\right)$, is likely caused by the existence of an increased number of turbulent spots that are at an earlier stage of their development and growth. 


\subsection{Summary}

This section has documented the effects of uniformly distributed surface roughness on boundary layer transition under low and elevated free-stream turbulence conditions. The development of the laminar boundary layer is found to be insensitive to roughness conditions typical of low pressure turbine blades of in-service gas turbines. However, surface roughness and free-stream turbulence are found to have comparable effects on the location of transition inception in separation bubbles. The sensitivity of the transition inception location to surface roughness is noted to increase with increased free-stream turbulence.

The rates of transition in separation bubbles are found to remain unaffected by the presence of rough surfaces, for all tested levels of free-stream turbulence. This is believed to be a result of the separated shear layer being located well above the peaks of the surface roughness elements in most instances. In the cases of attached flow transition, the roughness elements of the surface with the highest RMS roughness height considered protrude well into the region of the boundary layer where turbulent spot initiation occurs. This is observed to result in an increased transition rate.

The maximum amplification rate of instability waves occurs near the predicted fundamental Tollmien-Schlichting frequency in the attached flow test cases, and the majority of separation bubble test cases. The agreement between the observed and predicted instability frequencies provides further evidence that the mechanism of transition in these separation bubbles is dominantly of the Tollmien-Schlichting type. 
A significant increase in transition rate is observed for one of the pressure distributions tested. Evidence is provided to suggest that the transition process for this pressure distribution is driven by the inviscid Kelvin-Helmholtz instability mechanism. The very sudden transition process and large amplitude pre-transitional disturbances are consistent with the periodic shedding of vortical structures typical of the $\mathrm{K}-\mathrm{H}$ transition process. This shedding frequency matches the dominant frequency of the Tollmien-Schlichting waves prevailing in the separated shear layer. 


\section{Chapter 7}

\section{Large-Eddy Simulation of Transition in a Separation Bubble}

The majority of experimental studies on boundary layer transition have been based on single-point measurements using hot-wire anemometry (e.g. Schubauer \& Skramstad, 1948; Hatman \& Wang, 1999; Johnson, 1994; Solomon et al., 1996; Volino \& Simon, 1995), hot-film anemometry (e.g. Haueisen et al., 1997; Zhong et al., 2000; Anthony et al., 2004) and laser-doppler velocimetry (e.g. Stieger \& Hodson, 2004; Lang et al., 2004; Brendel \& Mueller, 1987). A common shortcoming of such measurement techniques is the absence of information regarding spatial correlation of the flow field. In separation bubbles, the unsteady development of the separated shear layer and the periodic shedding of vortical structures closely interact with the transition and reattachment processes. The availability of temporally synchronized information throughout the flow field would be beneficial to complete our understanding of this flow. Particle image velocimetry (PIV) is a relatively recent measurement technique that provides such information (Mills et al., 2003; Lang et al., 
2002; Bao \& Dallmann, 2004), but results with fine temporal resolution are yet to be produced with this technique.

The numerical study presented in this chapter has been undertaken with the objective of providing further insight into the physics of separation bubble transition, to complement the experimental studies of the present research program. To minimize any adverse effect of the issues associated with sub-grid modeling on this objective, the spatial and temporal resolution of the simulations are chosen to be significantly finer than those adopted in many published LES studies on transition flows, with the artificial dissipation inherent in the numerical algorithm providing the dissipation of turbulence energy at the level of unresolved scales. The level of resolution is similar to what is referred to as "Coarse DNS", or "Quasi-DNS" in the published literature (Dubois \& Bouchon, 1998; Spalart, 2000; Piomelli \& Balaras, 2002).

\section{$7.1 \quad$ Numerical Method}

\subsubsection{Computational Domain and Boundary Conditions}

The experiment that forms the basis of comparison for the present simulation is the low turbulence smooth surface test case conducted with the $C_{P 4}$ pressure distribution at $R e_{L}=350,000$ from Chapter 6. The computational domain used for the simulations is shown schematically in Fig. 7.1. The inflow boundary is placed $60 \mathrm{~mm}$ upstream of the separation location observed in the experiments. The mean velocity and turbulence intensity distributions specified on this boundary correspond to the experimental values. The very low free-stream turbulence intensity and the 


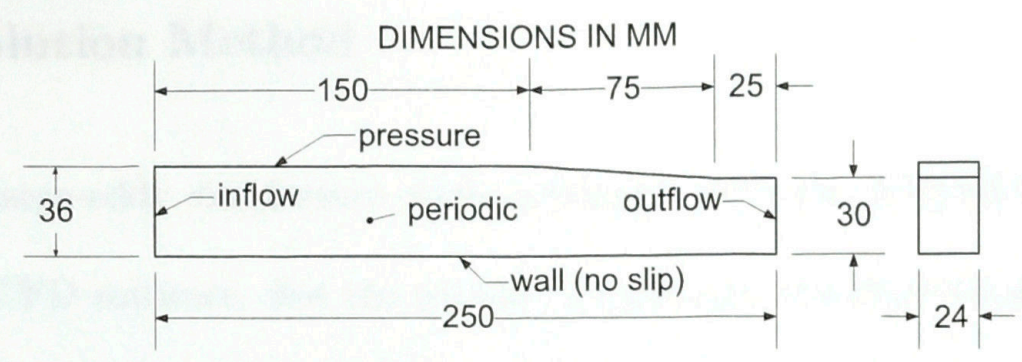

Figure 7.1: Schematic of the computational domain

laminar state of the boundary layer at this location eliminate the issues associated with the specification of transient inflow conditions typically required for large-eddy simulations.

The upper outflow boundary is placed well into the free-stream region of the flow field, and the static pressure distribution on this boundary is set to match the experimental values. The streamwise extent of the domain places the rear outflow boundary well downstream of the reattachment region. All flow properties are extrapolated to this boundary from the adjacent set of interior nodes with the restriction that the average static pressure on this boundary be consistent with the pressure specified along the upper outflow boundary.

In the spanwise direction, the domain is bounded by periodic boundaries, and is sized to be approximately four times the measured maximum thickness of the bubble. This spanwise extent is deemed sufficient to allow for natural development of large-scale three-dimensional transient features as the flow transitions to a turbulent state in the separated shear layer. 


\subsubsection{Solution Method}

The large-eddy simulations were performed with the ANSYS-CFX (v. 5.7) commercial CFD software. For the present simulations, the discretization of spatial derivatives was based on central differencing, and second-order accurate backward Euler integration was used to advance the solution in time. Higher order accuracy discretization schemes are preferable for large-eddy simulations, because the secondorder accuracy of the spatial and temporal discretizations may introduce excessive numerical dissipation, or possibly dispersion, into the solution. The temporal and spatial resolutions described in the next section are relatively fine for large-eddy simulation of the flow in question, and were chosen as such to help offset the dissipative effect of the discretization schemes. Convergence of the solution at each time increment was facilitated through inner iterations based on an algebraic multigrid scheme. Typically, 7 inner iterations were found to be sufficient to converge the solution by reducing the L2-norm of the residuals of the filtered continuity and momentum equations by three to four orders of magnitude.

\subsubsection{Computational Grid}

The spatial domain was discretized with a structured grid of 176, 52 and 17 nodes in the streamwise, wall-normal and spanwise directions, respectively. The nodes were evenly spaced in the spanwise and streamwise directions. In the wallnormal direction, the first node was placed to yield a $y^{+}$value of about 1 in the turbulent boundary layer downstream of the separation bubble, and the remaining nodes were distributed with an expansion factor of 1.1. These choices of node counts 
Table 7.1: Comparison of computational grids used in LES and DNS studies of transitional and turbulent flows

\begin{tabular}{|l|c|c|c|c|}
\hline Investigators & LES/DNS & $\Delta x^{+}$ & $\Delta z^{+}$ & $\begin{array}{c}\Delta y^{+} \\
\left(1^{\text {st }} \text { node }\right)\end{array}$ \\
\hline Current study & LES & 36 & 38 & $\sim 0.8$ \\
\hline Yang \& Voke & LES & $10-30$ & 9 & 1 \\
\hline Ducros et al. & LES & 44 & 41 & 1 \\
\hline ONERA $^{*}$ & LES & 60 & 25 & 2 \\
\hline FLUENT & LES & 1100 & 300 & 10 \\
\hline CERFACS* $^{*}$ & LES & 1500 & 100 & 200 \\
\hline Alam \& Sandham & DNS & $14-20$ & $\sim 1$ & $\sim 0.5$ \\
\hline Rai \& Moin & DNS & 28 & 10 & 1 \\
\hline
\end{tabular}

* included in the study of Mellen et al. (2002)

and distributions yielded reasonably isotropic grid cells in the separated shear layer, with $\Delta x / \Delta y$ and $\Delta z / \Delta y$ varying between 1.2 and 1.8 , hence avoiding excessive gridrelated distortion of the large-scale turbulence eddies.

In large-eddy simulations, the effect of the sub-grid scale turbulence model diminishes as the computational grid is refined, since in the limit all turbulence activity is resolved (DNS). In Table 1, the spatial grid of the present study is compared to published studies on LES and DNS of boundary layer transition (Rai \& Moin, 1993; Alam \& Sandham, 2000; Yang \& Voke, 2001; Ducros et al., 1996; Mellen et al., 2002). The current spatial grid is noted to correspond to a high resolution LES. In DNS, the node spacing of the grid should ideally be smaller than the Kolmogorov length scale, to allow adequate resolution of the smallest energy dissipating eddies. In practice however, accurate DNS has been achieved with node spacing as large as 2-3 times the Kolmogorov scale (e.g. Leonard, 1996; Yeung \& Pope, 1989). In the present study, the largest node spacing is approximately 20 times the Kolmogorov scale of the turbulent 
boundary layer downstream of the separation bubble. It can be argued that with most of the inertial subrange resolved by the computational grid, the specifics of the sub-grid scale turbulence model are of lesser importance. The authors have therefore opted to rely solely on the numerical dissipation of the algorithm in providing the energy drain from the smallest scale of resolved turbulence. The validity of this approach is demonstrated by the favourable comparison of the simulation results with experimental data presented in this chapter. It is worth noting that it is not an objective of the present work to evaluate the potential of using algorithm-specific numerical dissipation in LES of boundary layer transition. Rather, the computational grid was refined as much as the available computing resources permitted, to allow the use of LES as a numerical wind tunnel, without having to seek a precise sub-grid scale model.

A time-step size of $0.22 \mathrm{~ms}$ was chosen for the simulations, yielding a Courant number $(U \Delta t / \Delta x)$ of 1.7 based on the average node spacing and the free stream velocity at the inflow boundary. The chosen time-step size is smaller than the estimated Kolmogorov time scale, and was dictated by the numerical stability of the computations.

After convergence of the solution, the simulation was re-started and executed for an additional 1000 time steps after independently doubling the number of elements in each of the three grid directions, and halving the time-step. These four parametric studies confirmed insensitivity of the presented results to refinements in spatial and temporal resolution. 


\subsubsection{Initial Conditions}

The flow field in the computational domain was initialized with a velocity field matching the velocity distribution at the inflow boundary, and a streamwise pressure distribution matching the distribution fixed along the upper outflow boundary. Perturbations produced by the numerical algorithm resulted in a free-stream turbulence intensity of approximately $0.8 \%$. This is close to the experimental value of $0.5 \%$, and was sufficient to initiate the transition process without explicit introduction of background noise of a particular frequency spectrum into the computations.

The simulation was executed for 12,900 time steps corresponding to 2.84 seconds of temporal development of the flow field. With the noted initialization process, the results beyond the first 2000 time steps were established to be suitable for analysis of the flow field.

\subsection{Results and Discussion}

\subsubsection{Time-Averaged Flow Field}

The predicted time-averaged velocity in the separation bubble region is compared to the experimental results in Fig. 7.2. The streamwise location in this figure is referenced to the point of separation, $x_{s}$, to enable direct comparison of the results. The predicted location of separation is $21 \mathrm{~mm}$ downstream of the measured location (4 percent of the distance from the test-surface leading edge to the point of separation in the experiment). 15 percent variation in the local streamwise pressure gradient, which is within the precision of the experimental data, is sufficient to 


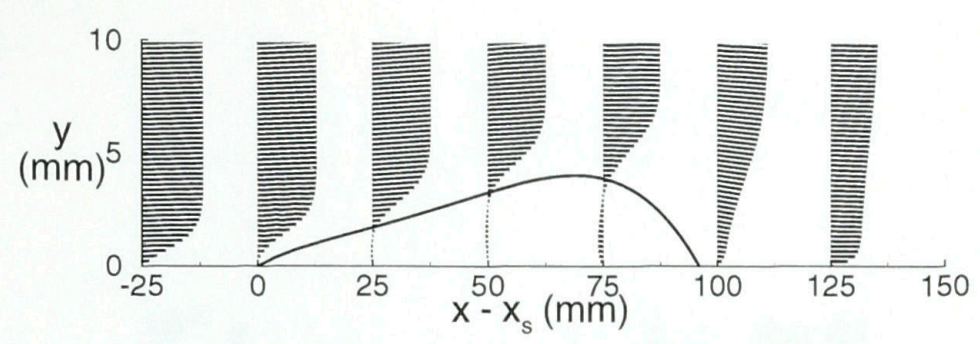

(a) LES

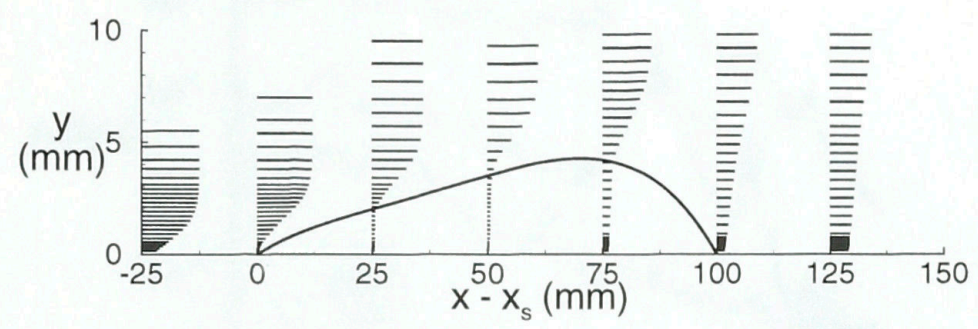

(b) Experiment

\section{Figure 7.2: Time-averaged velocity field in the region of the separation bubble (bubble contour shown by the dividing streamline)}

move the separation location by similar distances. This factor is judged to be the likely source of the noted discrepancy between the measured and predicted separation points.

As is evident from the path of the time-averaged dividing streamline, the length and thickness distribution of the separation bubble is captured accurately. The enhanced mass and momentum transfer resulting from transition to a turbulent state in the separated shear layer is key to the reattachment process. It is therefore unlikely to achieve the present level of prediction accuracy for the time-mean velocity field without capturing the main features of the transition process. The Fourier spectra of measured streamwise velocity fluctuations in the separated shear layer display a distinct peak at about $200 \mathrm{~Hz}$, starting near the separation location, and growing in amplitude with downstream distance (Fig. 7.3-a). The computed spectra 


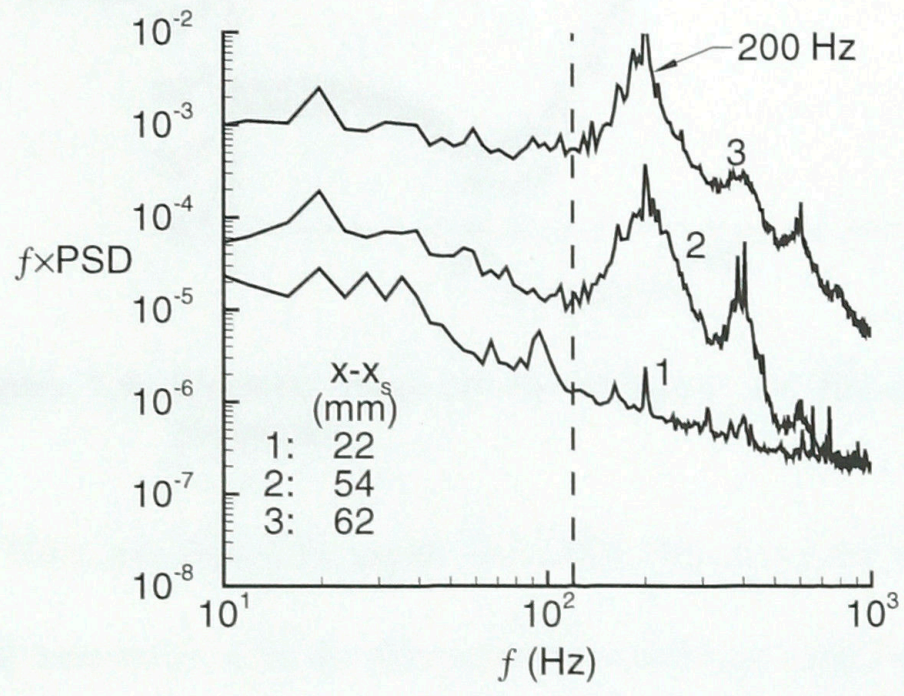

(a) Experiment

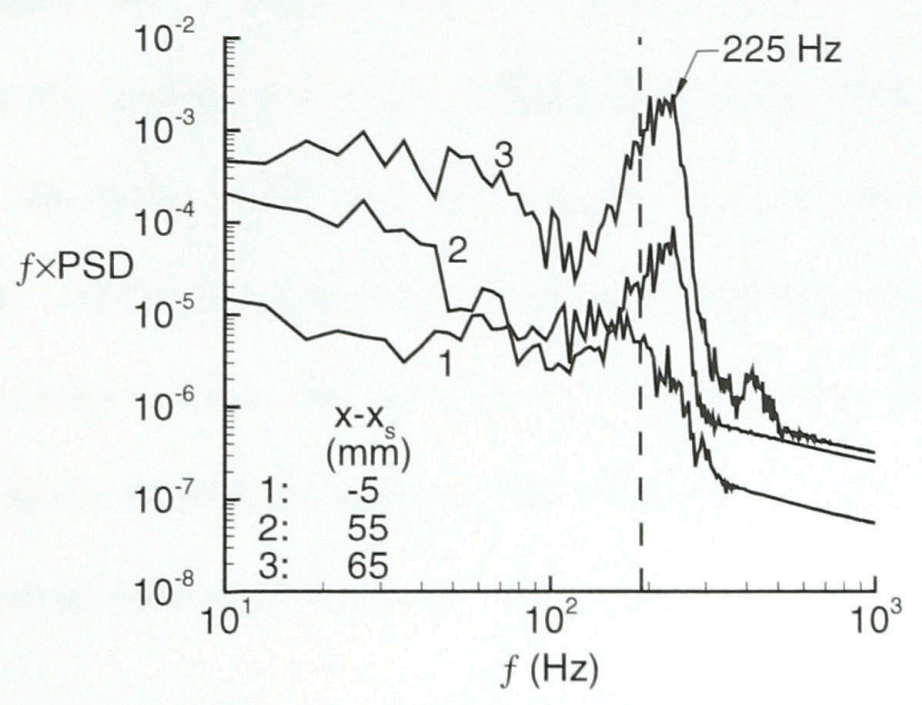

(b) LES

Figure 7.3: Power spectra of $u^{\prime} / U_{\text {ref }}$ at various locations in the separation bubble 


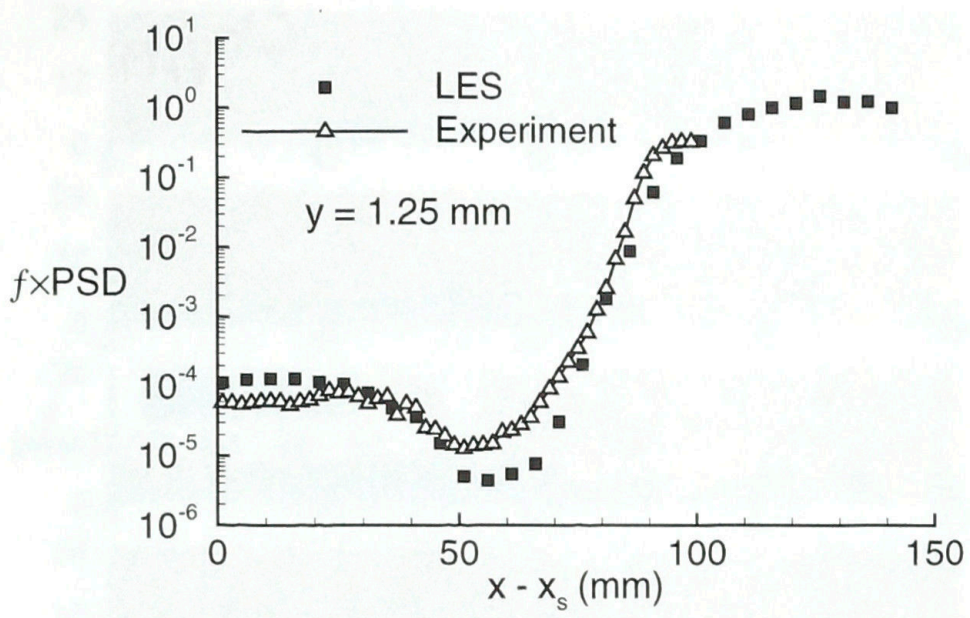

\section{Figure 7.4: Growth rates of disturbances at the dominant frequency}

given in Fig. 7.3-b are noted to agree well with the measured values. The present results are also consistent with the frequency of maximum amplification rate based on a correlation suggested by Walker (1989) for attached flow boundary layer transition, shown by a dashed line in Figs. 7.3-a \& 7.3-b. The peak observed in these $u^{\prime}$ spectra corresponds to the frequency at which Tollmien-Schlichting (T-S) waves grow at the highest rate. As shown in Fig. 7.4, this growth rate is predicted satisfactorily by the simulation. While the presence of Tollmien-Schlichting waves in the separated shear layer is clearly evident, the fact that no structures resembling turbulent spots are observed in the simulation suggests that breakdown occurs through a different instability, as is demonstrated in the next section.

\subsubsection{Transient Flow Development}

The temporal variation of the computed streamwise velocity field at mid-span of the computational domain is shown in Fig. 7.5 for one full period $(T=4.4 \mathrm{~ms})$ of the T-S cycle. The highly unsteady nature of the reattachment process is clearly 


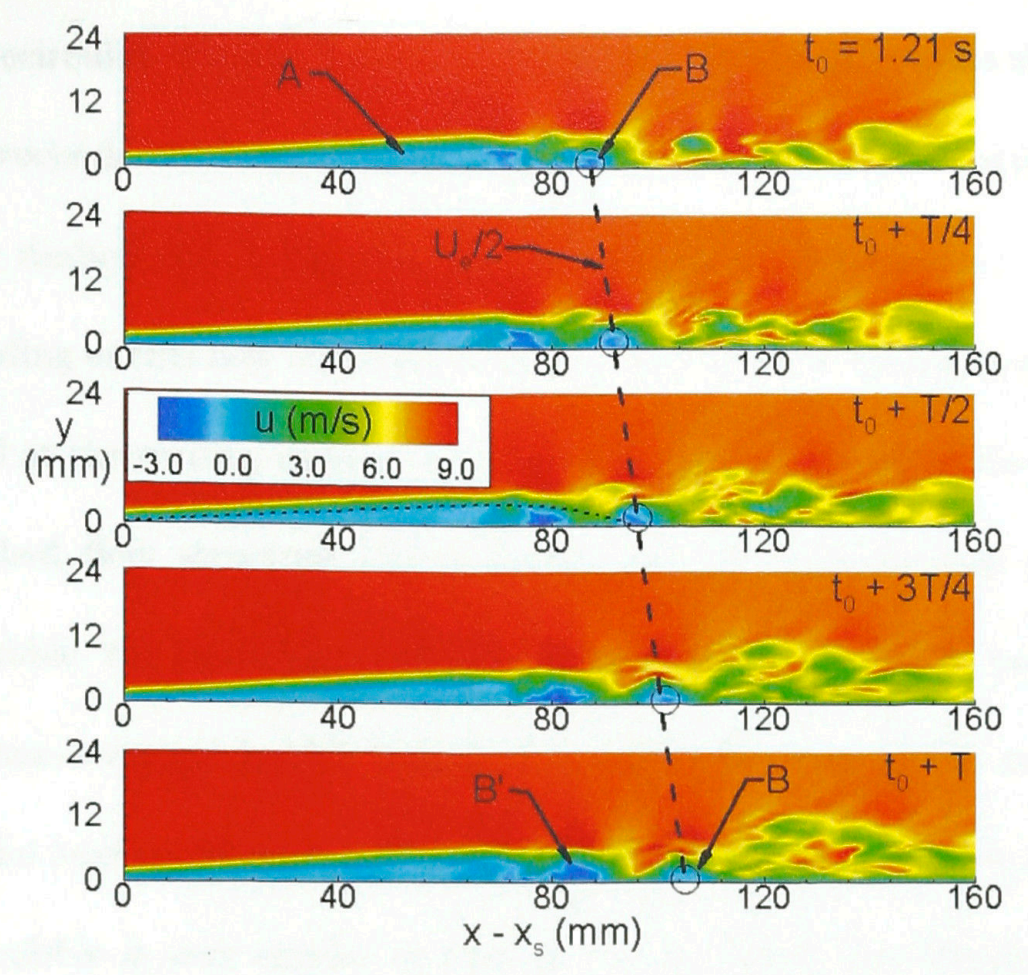

Figure 7.5: Streamwise velocity contours at mid-span during one full cycle of the dominant frequency (the dashed line at $t_{0}+T / 2$ represents the timeaveraged dividing streamline)

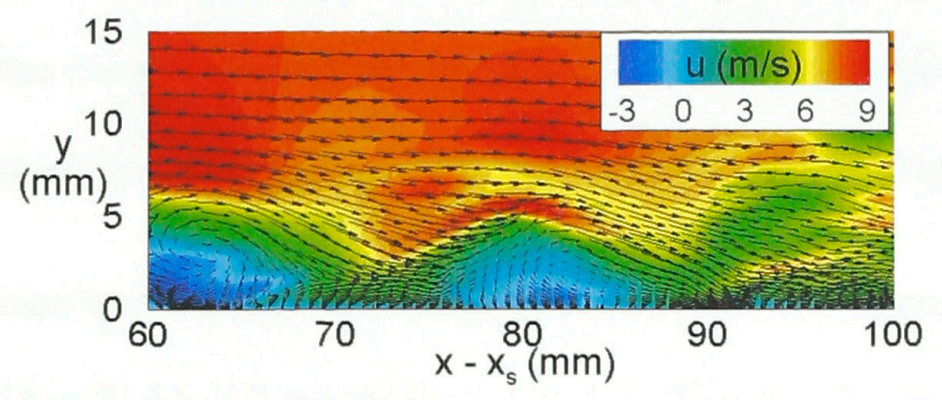

Figure 7.6: Magnified view of streamwise velocity contours at mid-span and $t=t_{0}+3 T / 4$ showing local reattachment of the flow

evident from the comparison of the velocity field in the five time planes shown.

The most obvious transient flow pattern is the periodic shedding of a small flow structure (B), containing reverse-flow from the main recirculation region (A) of the separation bubble. The most upstream location where this region B breaks from 
the primary recirculation zone A is $x-x_{s}=75 \mathrm{~mm}$. Subsequent to its shedding, this structure convects downstream at approximately half of the local free-stream velocity, shown by the dashed line in Fig. 7.5, and disappears at about $x-x_{s}=125 \mathrm{~mm}$. Periodic shedding of this flow structure results in two effective transient reattachment points located at the trailing ends of A and B (Fig. 7.6). If the one at the downstream end of the shed flow structure (B) is considered the reattachment point of the separation bubble, the shedding results in periodic movement of this point by about $30 \%$ of the time-averaged bubble length (Fig. 7.2). It is noted that at $t=t_{0}+T$, shedding of the next recirculating flow structure is about to take place $\left(\mathrm{B}^{\prime}\right)$, and the state of the bubble is very similar to that at $t=t_{0}$. Thus, the shedding frequency of this structure corresponds to the dominant frequency of the Tollmien-Schlichting waves prevailing in the separated shear layer. This result is consistent with the wavelength of the wavering developing in the streamlines of the separated shear layer prior to the formation and shedding of the structure labelled B (Fig. 7.7).

The instantaneous spanwise vorticity distribution corresponding to the time planes at $t_{0}$ and $t_{0}+T / 2$ in 7.5 are shown in Fig. 7.8. Figures 7.9 and 7.10 complement Fig. 7.8 by showing magnified views of the same vorticity field in the $x-y$ and $y-z$ planes, focusing on the region containing the recirculating flow structure (B) at time $t_{0}$. This structure consists of small vortices near the surface, with a concentrated region of vorticity located just above them (Fig. 7.10). The smaller vortices are most intense in the rear portion of the structure, and are highly three dimensional, as is evident from Fig. 7.9. 


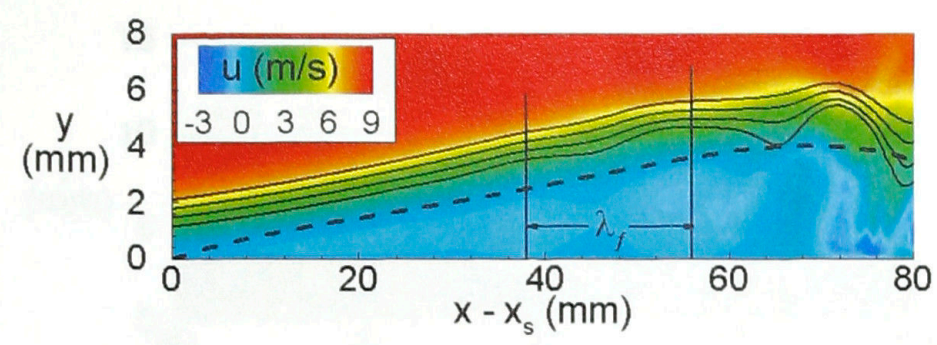

Figure 7.7: Instantaneous streamlines demonstrating shear layer wavering at the T-S wavelength $\left(\lambda_{f}\right)$

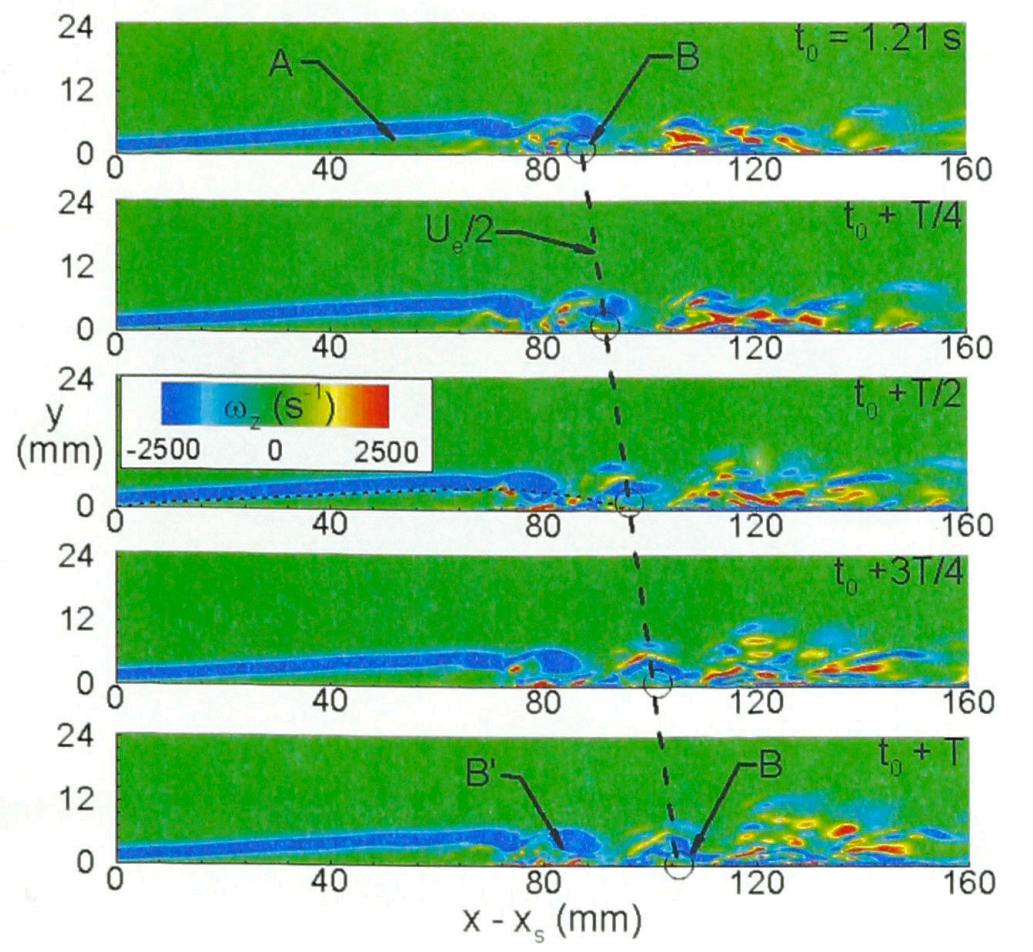

Figure 7.8: Contours of spanwise vorticity at mid-span at $t_{0}$ and $t_{0}+T / 2$

(the dashed line at $t_{0}+T / 2$ represents the timeaveraged dividing streamline)

Based on these results, the periodic shedding taking place at the trailing edge of the separation bubble is summarized schematically in Fig. 7.11-a. Wavering of the shear layer $(i)$ precedes the transient roll-up of vorticity in the separated shear layer, which begins upstream of the location of maximum bubble thickness (ii). The resultant vortex grows as it is convected along a path following the time-averaged diving streamline, and is shed at the point of maximum bubble thickness (iii). The 


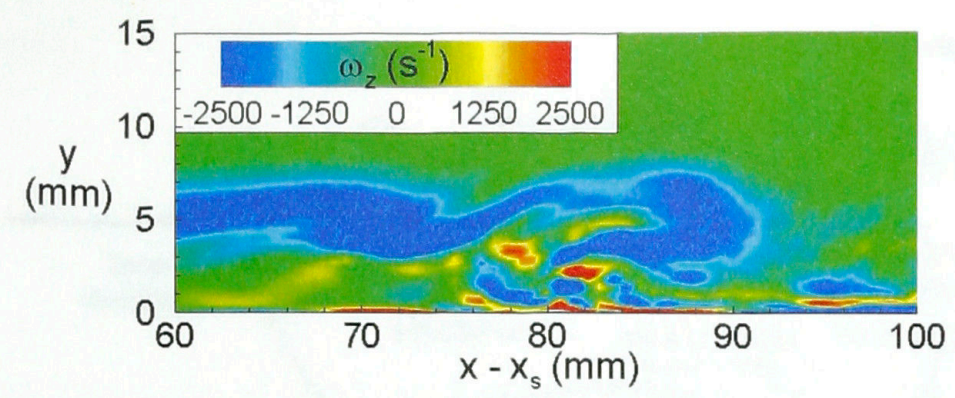

Figure 7.9: Magnified view of spanwise vorticity at midspan and $t=t_{0}$ (the dashed vertical line represents the location of the $x-z$ plane shown in Fig. 7.10)

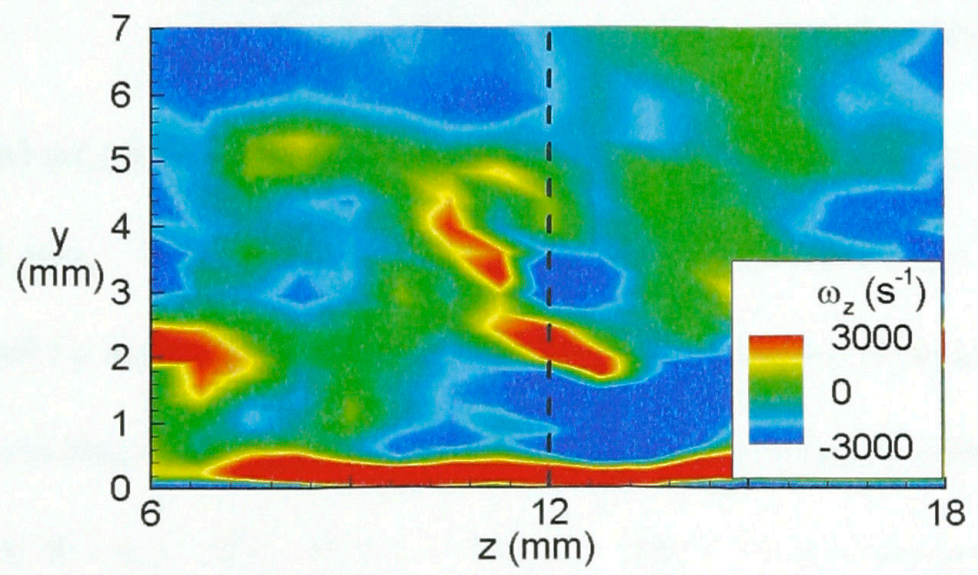

Figure 7.10: Spanwise vorticity at $x-x_{s}=81 \mathrm{~mm}$ and $t=t_{0}$ (the dashed vertical line represents the location of the $x-y$ plane shown in Fig. 7.9)

shedding process is accompanied by entrainment of small three-dimensional vortical structures from within the bubble $(i v)$. This flow development is somewhat different from an earlier proposal by Hatman \& Wang $(1998,1999)$, in which the shed vortex originates from within the bubble, and the entrained vortices seen near the surface in the current results are replaced by vortices ejected through the separated shear layer under the influence of the shed vortex (Fig. 7.11-b).

Examination of the velocity fluctuations in the $x, y$, and $z$ directions suggests that breakdown to turbulence occurs in the lower portion of the shear layer, shortly 


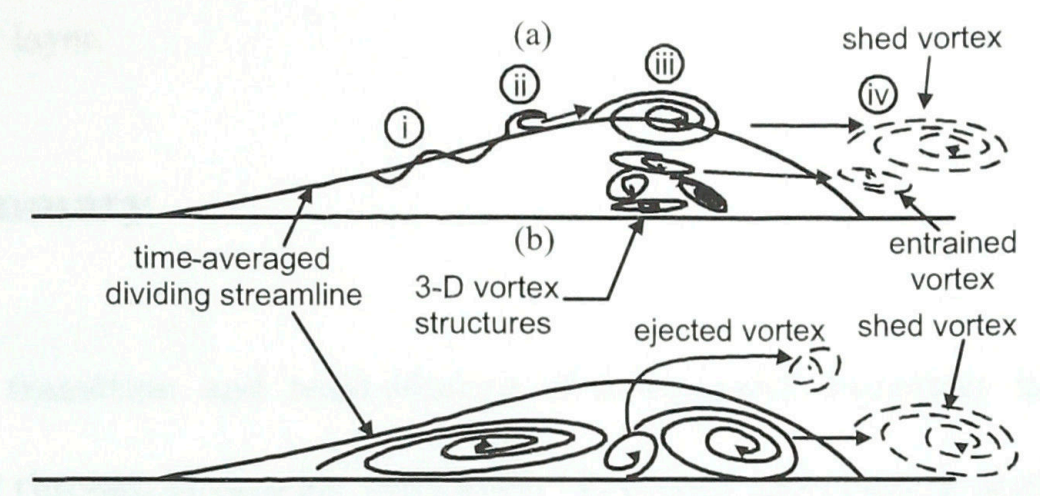

Figure 7.11: Schematic representation of the vortex shedding process superimposed on the timeaveraged dividing streamline: (a) Present results; (b) Hatman \& Wang (1999) model

before the shed structure (B) breaks from the main recirculation region (A) at about $x-x_{s}=70 \mathrm{~mm}$. The roll-up of the separated shear layer into a concentrated vortex, followed by breakdown to turbulence in this structure, is typical for transition driven by Kelvin-Helmholtz (K-H) instability (Spalart \& Strelets, 2000; Yang \& Voke, 2001; Bernal \& Roshko, 1986; Moser \& Rogers, 1993). In the present case, the timeaveraged velocity profile just upstream of the location of breakdown to turbulence is well represented by a hyperbolic tangent function. For this shape of velocity profile, Chandrasekhar (1961) computed the range for K-H instability to be $0<\kappa d<1$, where $\kappa$ is the wave number of the dominant disturbance frequency, and $d$ is a length scale determined by the hyperbolic tangent curve fit to the velocity distribution (equal to approximately $1 / 4$ of the shear layer thickness). For the present case, $\kappa d$ is determined to be about 0.5 , with $\kappa$ being the wave number of the T-S waves developing in the separated shear layer. The present case therefore demonstrates that even in separated shear layers where transition to turbulence occurs due to $\mathrm{K}-\mathrm{H}$ instability, the transition process can remain significantly affected by the T-S waves developing 
in the shear layer.

\subsection{Summary}

The transition and reattachment of a separated boundary layer has been investigated through large-eddy simulation. Excellent agreement is obtained between the experimental hot-wire measurements and the simulation in terms of the timeaveraged length and thickness of the separation bubble and frequency spectra of the velocity fluctuations. The computational study complements the experimental results by identifying certain phenomena that were not possible to observe directly in the experiments. Specifically, in this particular case, breakdown to turbulence is shown to take place through the Kelvin-Helmholtz instability. Periodic shedding of vorticity associated with this instability is shown to occur at a frequency that matches the dominant Tollmien-Schlichting frequency in the separated shear layer. The streamwise oscillation in the reattachment location caused by periodic vortex shedding is determined to be a significant fraction of the time-averaged bubble length. 


\section{Chapter 8}

\section{Modeling Boundary Layer Transition}

Since the concept of the turbulent spot was initially proposed by Emmons (1951), extensive experimental research efforts have been dedicated to uncovering the mechanisms leading to transition inception (e.g. Abu-Ghannam \& Shaw, 1980; Johnson \& Dris, 2000; Kachanov, 2000; Klebanoff et al., 1961), and the process of growth and merging of turbulent spots into a fully turbulent boundary layer (e.g. Gostelow et al., 1996; Johnson \& Fashifar, 1994; Narasimha, 1985). Studies such as these have increased our understanding of the transition process, and have identified the flow parameters that significantly affect this process, namely free-stream turbulence intensity (e.g. Blair, 1982; Volino \& Hultgren, 2001) and turbulence length scale (e.g. Johnson \& Ercan, 1997, and see also Chapter 4), streamwise pressure gradients (Fraser et al., 1994; Gostelow \& Blunden, 1989; Sharma et al., 1988), streamline curvature (Kim et al., 1992; Schultz \& Volino, 2001), periodic impingement of wakes (Stieger \& Hodson, 2004; Dong \& Cumpsty, 1990-b; Schobeiri \& Radke, 
1994), and surface roughness (Cummings \& Bragg, 1996; Pinson \& Wang, 1997).

As was discussed in Chapter 2, the computing requirements of DNS and LES are still beyond the reach of present-day technology, particularly for complex, three-dimensional, unsteady flows of practical engineering interest. Furthermore, transition models based on low Reynolds number turbulence models have only met with moderate success in predicting transitional flows. Thus, for the foreseeable future, semi-empirical tools appear to be the most accurate and time efficient means for modeling turbulent spot inception and propagation. This portion of the present study proposes models that account for the effects of a broader range of flow and geometric parameters, calibrated against an extensive set of experimental data.

\subsection{Description of Experiments}

The data compiled for this portion of the study have been extracted mostly from the experiments presented in Chapters 4, 5, and 6, which are identified by the abbreviation RY. In order to confirm the absence of any trends in these results that may be unique to the wind tunnel and instrumentation used, results from several other research facilities have been included in the data set. The additional data are those published by Volino and co-workers (Volino, 2002-b; Volino \& Hultgren, 2001; Volino, Schultz, \& Pratt, 2001), denoted by the abbreviations V, VH \& VSP, respectively, and Devasia (1974), abbreviated as DV. The data set encompasses a range of flow Reynolds numbers, pressure gradients, free-stream turbulence levels, and surface roughness conditions that are typical of gas turbine applications, and 
includes cases with attached flow (Chapter 4, Volino \& Hultgren, 2001; Volino et al., 2001; Devasia, 1974, and separation bubble transition (Chapters 5 \& 6 and Volino, 2002-b).

With the exception of the most recent experiments of Volino (V) (2002-b), the noted wind-tunnel transition studies were performed using a flat test surface, with a well defined location for the beginning of the boundary layer development. In each case, streamwise pressure distributions were imposed upon the test surface through the use of a contoured test-section ceiling. The ceiling was configured to yield pressure gradients that are favourable (Volino \& Hultgren, 2001; Devasia, 1974; Volino et al., 2001), adverse (Chapter 4 and Devasia (1974)), or resemble those typically encountered on the suction surface of turbine blades (Chapters 4, 5, and 6, and Volino (2002-b)). The test surfaces were wide enough to ensure two-dimensional flow development at the spanwise locations of the boundary layer measurements. Variations in free-stream turbulence were achieved by the use of turbulence-generating grids, placed sufficiently upstream of the test surface to yield isotropic and homogeneous turbulence. Surface roughness variations, which are limited to the test cases of the present study (Chapter 6) of the present authors, were achieved through the use of commercially available materials that provided random roughness patterns.

The experiments of Volino (V) were performed in a single-passage rectilinear cascade test-section. Through comparison of the transition trends prevailing on this blade to those observed on flat surfaces in the remainder of the studies considered 
Table 8.1: Summary of experimental configurations

\begin{tabular}{|l|l|c|c|c|c|c|}
\hline Study & $\begin{array}{l}\text { Test section } \\
\text { config. }\end{array}$ & $\begin{array}{c}R e_{L} \\
\times 10^{5}\end{array}$ & $\begin{array}{c}T u_{\text {ref }} \\
\%\end{array}$ & $\begin{array}{c}\text { Rough } \\
\text { surface }\end{array}$ & $\begin{array}{c}C_{P} \\
\text { dist. }\end{array}$ & $\begin{array}{c}\text { Hot-wire } \\
\text { type }\end{array}$ \\
\hline RY & Flat plate & $3.5-9.3$ & $0.5-9.0$ & $\checkmark$ & Adv., Blade & Single \\
\hline VSP & Flat plate & 3.5 & 8.7 & $\times$ & Fav. & X-wire \\
\hline V & Cascade & $1.0-3.0$ & 8.7 & $\times$ & Blade & X-wire \\
\hline VH & Flat plate & $0.5-3.0$ & 7 & $\times$ & Fav. & Single \\
\hline DV & Flat plate & $13.9-18.3$ & $1.4-3.9^{\mathrm{a}}$ & $\times$ & Fav., Adv. & Single \\
\hline
\end{tabular}

a: Estimated from turbulence grid and test section geometry

here, the effects of convex surface curvature on boundary layer transition may be inferred. Only three of the ten test cases published by Volino are included in the current study, namely those with high free-stream turbulence and Reynolds numbers based on the suction surface length of 100,000 to 300,000 . In the remaining seven test cases, the transition process occurred too rapidly to yield intermittency distributions with sufficient streamwise resolution for the purposes of the present study.

The noted experimental data sets are summarized in Table 8.1. The relevant flow parameters from previous chapters of this document are repeated in Tables 8.2 and 8.3. Four different pressure distributions were investigated in these studies: the pressure distributions identified as $C_{P 1}, C_{P 2}$, and $C_{P 4}$ are similar to those prevailing on the suction side of low-pressure turbine blades. The initial accelerations for these three pressure distributions are approximately the same, but the subsequent adverse pressure gradient is strongest in the case of the $C_{P 1}$ distribution, and weakest in the case of the $C_{P 4}$ pressure distribution. The designation $C_{P 3}$ corresponds to a nearly constant adverse pressure gradient that begins at the leading edge of the test surface, and is milder than that encountered in the downstream portions of the $C_{P 1}, C_{P 2}$, and 
$C_{P 4}$ pressure distributions.

It should be noted that in the experiments with the $C_{P 4}$ pressure distribution, a Kelvin-Helmholtz transition process occurred (as discussed in Chapters 6 \& 7). Since the transition length model presented in this section is based on the hypothesis of production and growth of turbulent spots, these test cases should not be used in the development of such a model. However, this does not prevent the use of these test cases in developing a model for predicting transition inception in separation bubbles, and the relevant experimental data are therefore included separately in Table 8.4.

\subsection{Proposed Model}

\subsubsection{Location of Transition Inception}

\subsubsection{Attached Flow}

As shown in Fig. 8.1, the transition inception locations from earlier phases of the present research, covering a range of favourable and adverse pressure gradients, and free-stream turbulence levels between $1.7 \%$ and $3.3 \%$, are predicted reasonably well by the model of Abu-Ghannam \& Shaw (1980), given by Eq. 2.5 (repeated below), and shown by the solid lines in Fig. 8.1.

$$
R e_{\theta_{t s}}=163+e^{F\left(\lambda_{\theta}\right)\left(1-\frac{\overline{T u} \%}{6.91}\right)}
$$


Table 8.2: Flow parameters at transition inception attached flow test cases

\begin{tabular}{|c|c|c|c|c|c|c|c|c|}
\hline $\begin{array}{c}C_{P} \\
\text { dist. }\end{array}$ & $\begin{array}{l}R e_{L} \\
\times 10^{3}\end{array}$ & $\begin{array}{l}\frac{k_{r m s}}{\theta_{t s}} \\
\times 10^{-3}\end{array}$ & $\begin{array}{c}\text { Tu ref } \\
\%\end{array}$ & $\begin{array}{c}\text { TF } \\
\%\end{array}$ & $\begin{array}{c}\lambda_{\theta_{t s}} \\
\times 10^{-2}\end{array}$ & $R e_{\theta_{t s}}$ & $H_{t s}$ & $\log (N)$ \\
\hline 1 & 350 & 2.0 & 6.4 & 14.0 & 9.5 & 167 & 1.7 & -4.1 \\
\hline 1 & 350 & 347 & 4.5 & 11.2 & -12.2 & 312 & 2.2 & -0.1 \\
\hline 1 & 470 & 2.3 & 4.4 & 11.2 & 11.4 & 191 & 1.8 & -3.6 \\
\hline 1 & 470 & 1.6 & 6.8 & 15.7 & 12.8 & 199 & 1.7 & -3.8 \\
\hline 1 & 470 & 131 & 2.4 & 5.3 & 5.2 & 278 & 1.9 & -3.5 \\
\hline 1 & 470 & 89 & 4.1 & 10.3 & -3.1 & 245 & 2.0 & -2.4 \\
\hline 1 & 470 & 141 & 4.1 & 10.2 & -1.7 & 286 & 2.0 & -2.4 \\
\hline 1 & 470 & 411 & 2.5 & 5.8 & -20.5 & 354 & 2.5 & -0.2 \\
\hline 1 & 470 & 568 & 4.5 & 11.2 & -8.1 & 266 & 2.1 & -1.2 \\
\hline 2 & 350 & 1.9 & 8.9 & 20.8 & 10.1 & 177 & 1.7 & -3.8 \\
\hline 2 & 470 & 2.2 & 6.8 & 15.1 & 11.1 & 171 & 1.8 & -3.9 \\
\hline 2 & 470 & 1.8 & 9.0 & 21.0 & 13.7 & 185 & 1.6 & -3.8 \\
\hline 3 & 350 & 0.9 & 2.3 & 6.7 & -3.4 & 227 & 2.6 & -2.4 \\
\hline 3 & 350 & 0.9 & 3.7 & 8.0 & -4.6 & 226 & 2.6 & -2.7 \\
\hline 3 & 350 & 1.1 & 4.3 & 11.3 & -3.4 & 176 & 2.3 & -3.0 \\
\hline 3 & 350 & 1.8 & 6.2 & 13.2 & -1.4 & 122 & 2.2 & -3.8 \\
\hline 3 & 470 & 1.2 & 2.2 & 6.0 & -4.3 & 239 & 2.6 & -2.8 \\
\hline 3 & 470 & 2.9 & 3.9 & 9.4 & -5.6 & 258 & 2.4 & -3.0 \\
\hline 3 & 470 & 1.9 & 4.4 & 11.4 & -1.3 & 141 & 2.2 & -3.6 \\
\hline 3 & 470 & 2.6 & 6.1 & 13.1 & -0.8 & 98 & 2.1 & -3.8 \\
\hline 3 & 650 & 0.9 & 0.7 & 0.9 & -6.6 & 364 & 2.9 & -1.7 \\
\hline 3 & 650 & 1.7 & 2.5 & 6.3 & -3.3 & 216 & 2.4 & -2.9 \\
\hline 3 & 650 & 2.0 & 3.8 & 9.0 & -3.2 & 226 & 2.2 & -2.8 \\
\hline 3 & 650 & 2.7 & 4.6 & 11.9 & -0.7 & 139 & 2.1 & -3.6 \\
\hline 3 & 930 & 1.5 & 0.5 & 0.6 & -5.1 & 351 & 2.8 & -1.5 \\
\hline 3 & 930 & 1.6 & 3.8 & 9.6 & -0.6 & 175 & 2.1 & -3.4 \\
\hline 3 & 930 & 3.2 & 6.5 & 14.0 & -1.3 & 168 & 1.9 & -3.7 \\
\hline
\end{tabular}

$$
\begin{array}{cc}
F\left(\lambda_{\theta}\right)=6.91+12.75 \lambda_{\theta}+63.64 \lambda_{\theta}{ }^{2} & \left\{\lambda_{\theta} \leq 0\right\} \\
F\left(\lambda_{\theta}\right)=6.91+2.48 \lambda_{\theta}+12.27 \lambda_{\theta}{ }^{2} & \left\{\lambda_{\theta}>0\right\}
\end{array}
$$

This agreement is achieved without having to adjust the value of $R e_{\theta_{t s}}$ predicted 
Table 8.3: Flow parameters at separation and transition inception - separation bubble test cases (all test cases are for the $C_{P 1}$ pressure distribution)

\begin{tabular}{|c|c|c|r|r|r|r|r|r|r|}
\hline $\begin{array}{c}R e_{L} \\
\times 10^{3}\end{array}$ & $\begin{array}{c}\frac{k_{r m s}}{\theta_{s}} \times 10^{-3} \\
\times 3 u_{\text {ref }}\end{array}$ & $\begin{array}{c}\text { TF } \\
\%\end{array}$ & $\begin{array}{c}\lambda_{\theta_{t s}} \\
\times 10^{-2}\end{array}$ & $R e_{\theta_{t s}}$ & $\begin{array}{c}R e_{s-t s} \\
\times 10^{3}\end{array}$ & $H_{s}$ & $H_{t s}$ & $\log (N)$ \\
\hline 350 & 1.3 & 0.6 & 1.7 & -3.3 & 263 & 34 & 3.1 & 8.1 & 0.5 \\
\hline 350 & 1.4 & 0.6 & 1.2 & -6.4 & 258 & 36 & 3.2 & 8.7 & 0.3 \\
\hline 350 & 1.3 & 2.2 & 5.9 & -10.1 & 252 & 32 & 3.0 & 6.4 & 0.6 \\
\hline 350 & 1.3 & 4.2 & 8.9 & -8.4 & 285 & 23 & 3.0 & 4.3 & 0.1 \\
\hline 350 & 62 & 0.7 & 1.4 & -13.8 & 257 & 30 & 3.0 & 4.2 & 0.9 \\
\hline 350 & 66 & 2.2 & 5.6 & -3.0 & 258 & 31 & 3.0 & 5.7 & 0.3 \\
\hline 350 & 56 & 4.4 & 10.8 & -6.5 & 288 & 7 & 3.0 & 3.3 & -0.6 \\
\hline 350 & 110 & 0.7 & 1.3 & -10.2 & 257 & 35 & 3.1 & 5.6 & 0.5 \\
\hline 350 & 97 & 2.2 & 5.4 & -13.9 & 287 & 19 & 3.0 & 4.5 & -0.2 \\
\hline 350 & 94 & 4.2 & 10.3 & -13.4 & 314 & 4 & 2.9 & 3.2 & -0.4 \\
\hline 350 & 347 & 0.7 & 1.7 & -13.8 & 286 & 17 & 3.5 & 4.7 & 0.2 \\
\hline 350 & 340 & 2.4 & 6.2 & -24.9 & 321 & 4 & 3.7 & 3.6 & -0.2 \\
\hline 470 & 1.6 & 0.4 & 0.7 & -5.8 & 306 & 45 & 3.1 & 6.8 & 0.8 \\
\hline 470 & 1.6 & 0.4 & 0.6 & -7.7 & 305 & 47 & 3.0 & 7.3 & 0.7 \\
\hline 470 & 1.5 & 2.3 & 5.9 & -11.1 & 309 & 38 & 3.0 & 5.2 & 0.6 \\
\hline 470 & 1.5 & 3.4 & 8.4 & -9.0 & 312 & 31 & 2.9 & 4.4 & 0.3 \\
\hline 470 & 78 & 0.6 & 0.8 & -13.4 & 316 & 38 & 3.1 & 5.7 & 0.9 \\
\hline 470 & 49 & 2.3 & 5.4 & -32.7 & 444 & 17 & 2.8 & 3.2 & 0.3 \\
\hline 470 & 98 & 0.5 & 0.7 & -16.2 & 376 & 35 & 3.0 & 5.3 & 0.3 \\
\hline 470 & 405 & 0.6 & 0.9 & -15.8 & 338 & 8 & 3.5 & 4.2 & -0.1 \\
\hline
\end{tabular}

Table 8.4: Flow parameters at separation and transition inception - separation bubble test cases (all test cases are for the $C_{P 4}$ pressure distribution)

\begin{tabular}{|c|c|c|c|c|c|c|}
\hline $\begin{array}{c}R e_{L} \\
\times 10^{3}\end{array}$ & $\begin{array}{c}\frac{k_{r m s}}{\theta_{s}} \\
\times 10^{-3}\end{array}$ & $\begin{array}{c}\text { Tu } u_{\text {ref }} \\
\%\end{array}$ & $\begin{array}{c}\text { TF } \\
\%\end{array}$ & $\begin{array}{c}\lambda_{\theta_{t s}} \\
\times 10^{-2}\end{array}$ & $R e_{\theta_{t s}}$ & $\begin{array}{c}R e_{s-t s} \\
\times 10^{3}\end{array}$ \\
\hline 145 & 1.0 & 0.6 & 1.1 & -10.6 & 169 & 24 \\
\hline 145 & 231 & 0.9 & 1.3 & -4.0 & 196 & 15 \\
\hline 350 & 1.4 & 0.8 & 1.7 & -20.6 & 309 & 38 \\
\hline 350 & 62 & 0.8 & 1.7 & -12.1 & 283 & 34 \\
\hline 350 & 388 & 0.7 & 1.2 & -14.6 & 271 & 22 \\
\hline 470 & 1.8 & 0.6 & 0.9 & -14.4 & 329 & 43 \\
\hline 470 & 67 & 0.5 & 1.0 & -14.7 & 345 & 31 \\
\hline
\end{tabular}




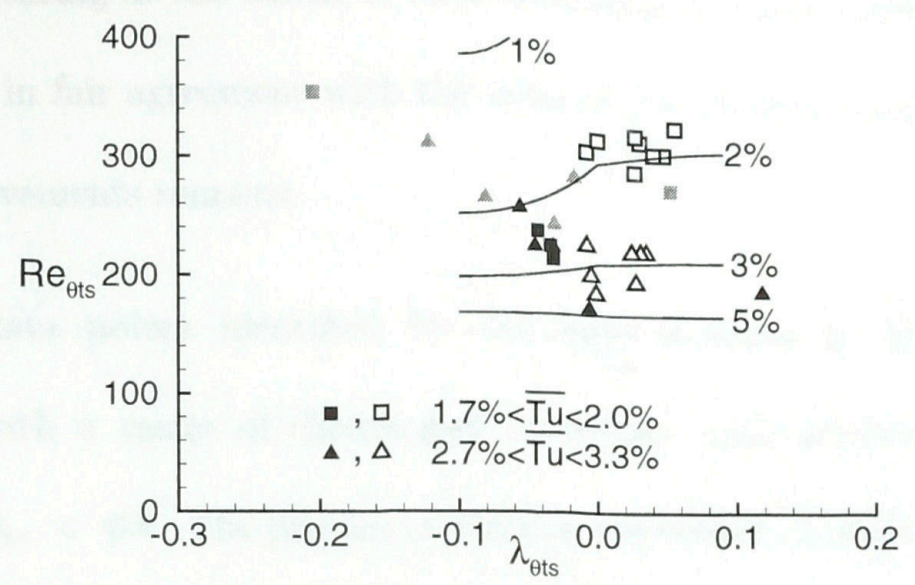

Figure 8.1: Comparison of present experimental results (filled symbols) to the experimental data (hollow symbols) and correlation (lines) of AbuGhannam and Shaw (the present rough surface test cases are shown in grey)

by the model to account for a bias created by the measurement technique of AbuGhannam \& Shaw (1980), as suggested by Fraser et al. (1994) and Dey \& Narasimha (1988). It must be recognized however, that the scatter in the data observed in Fig. 8.1, which is comparable to the scatter of the original data set of Abu-Ghannam \& Shaw (1980), translates into an uncertainty in the predicted $R e_{\theta}$ at transition onset of 15 to 20 percent. This level of precision may be inadequate in certain instances. For example, for the separation bubble transition measurements of Chapters 5 and 6, where the streamwise pressure distribution is typical of the suction side of a turbine blade, the model of Abu-Ghannam \& Shaw (1980) would predict attached flow transition instead. Such a difference could be critical in estimating profile losses and heat transfer characteristics of a turbine blade. The transition process in separation bubbles was shown by Yaras (2001) to be sensitive to the pressure gradient history of the boundary layer prior to separation. Similar effects are also likely present in instances of attached flow transition that cannot be accounted for with the parameters 
currently appearing in the model of Abu-Ghannam \& Shaw (1980). Thus, although this model is in fair agreement with the data of the present study, the potential for further improvements remains.

The data points identified by the grey symbols in Fig. 8.1 correspond to surfaces with a range of distributed roughness with heights of approximately $0.1<k_{r m s} / \theta_{t s}<0.6$. The physics of the observed effect of surface roughness on the location of transition inception is discussed in Chapter 6. Although a significant effect of roughness on $R e_{\theta_{t s}}$ is evident, the relative sparseness of the data set prevents reliable improvements to the model of Abu-Ghannam \& Shaw (1980). Further measurements are needed before attempting such improvements that would make the model more relevant for the performance prediction of in-service gas turbine blades.

\subsubsection{Separation Bubbles}

For transition in separation bubbles, the correlation of Yaras (2002, Eq. 2.15) provides reliable estimates of the location of transition inception. This model captures the trend of reduced sensitivity of $R e_{t s}$ to free-stream turbulence as $T u$ increases. However, $R e_{x}$ typically does not vary significantly between separation and transition inception, due to the decreasing free-stream velocity partially offsetting the increase in the distance from the leading edge. As a result, it is difficult to capture slight upstream movements of the transition inception point through a correlation based on $R e_{t s}$. This can be better accomplished if the dependent parameter $R e_{t s}$ is replaced with the Reynolds number based on the distance between the separation and transition inception locations $\left(R e_{s-t s}\right)$. The proposed correlation, shown in Fig. 8.2, is given by: 


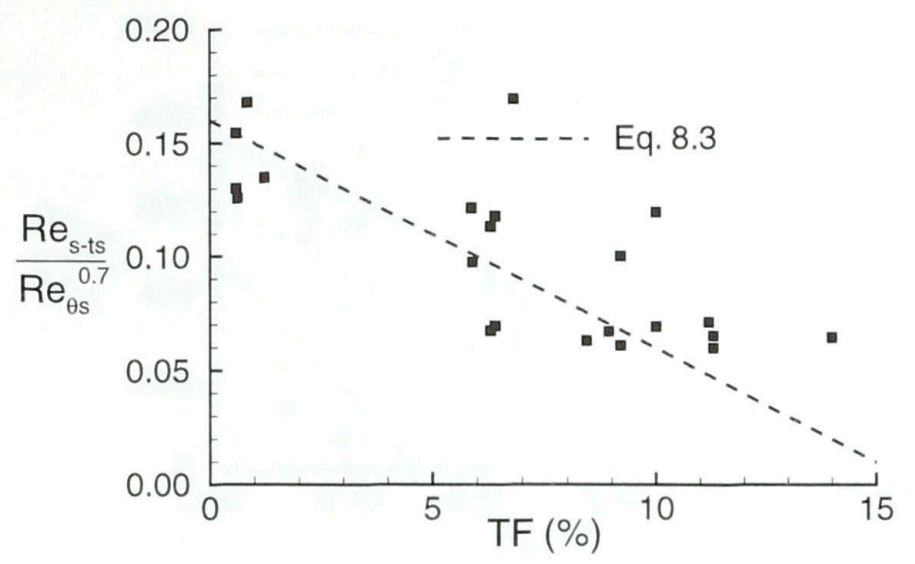

Figure 8.2: Sensitivity of the transition inception location in the separation bubble to free-stream turbulence (smooth surfaces)

$$
R e_{s-t s}=(785-30 T F(\%)) R e_{\theta_{s}}^{0.7}
$$

Although Eq. 8.3 may not appear to be the best fit to the smooth surface data shown in Fig. 8.2, this model was been developed in the context of both rough and smooth surface measurements, as explained below.

The effect of surface roughness height is demonstrated in terms of the parameters of the proposed model in Fig. 8.3. The upstream movement of the transition point with increased surface roughness height is clearly evident, and the rate of this movement appears to increase somewhat with free-stream turbulence. The proposed transition inception model (Eq. 8.3) can be made to account for surface roughness effects through the following modification:

$$
R e_{s-t s}=\left(785-30 T F(\%)-\left(1400+25 e^{0.45 T F(\%)}\right) \frac{k_{r m s}}{\theta_{s}}\right) R e_{\theta_{s}}^{0.7}
$$




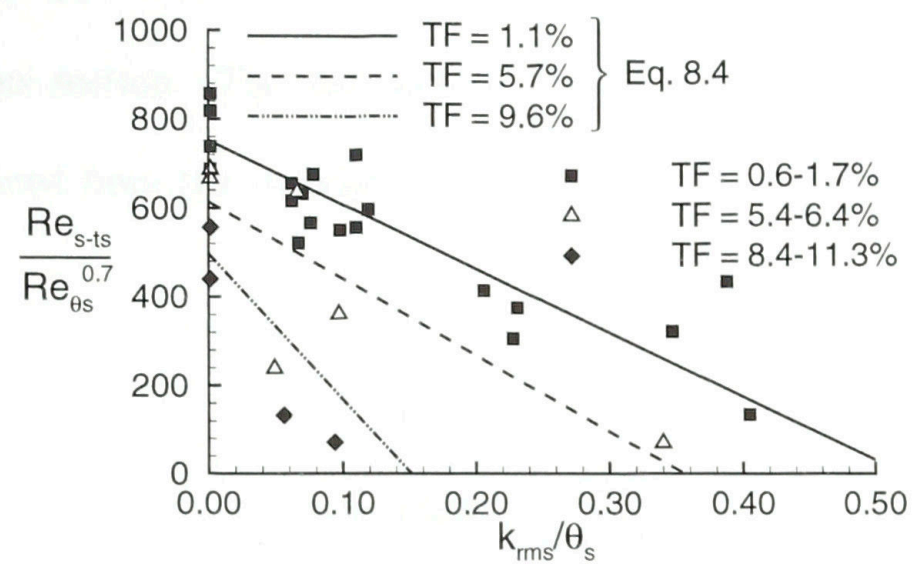

Figure 8.3: Sensitivity of the transition inception location to surface roughness and free-stream turbulence

\subsubsection{Length of Transition}

The transition length model is based on the expression for the intermittency distribution developed by Solomon et al. (1996, Eq. 2.17), repeated here for convenience:

$$
\gamma(x)=1-e^{-n \int_{x t s}^{x} \frac{\sigma}{\tan (\alpha) U} d x \int_{x_{t s}}^{x} \tan (\alpha) d x}
$$

As was noted in Section 2.4.2, this expression has its roots in the work of Emmons (1951), and has been brought to the present level of formulation through the studies of Narasimha (1957, 1985), Chen \& Thyson (1971), and Solomon et al. (1996) This formulation has been demonstrated to be consistent with the physics of the transition process, and is therefore adopted in the present model. The length of the transition zone is quantified through the distance over which Eq. 8.5 yields intermittency values greater than 0 and less than 1 . The location of transition 
inception, $x_{t s}$, appearing in this expression is obtained from the models proposed in the previous section. The parameters $\sigma$ and $\alpha$ representing the spot spreading rate are obtained from the correlations of D'Ovidio et al. (2001) and Solomon et al. (1996):

$$
\begin{gathered}
\sigma=0.024+\frac{0.604}{1+5 e^{66 \lambda_{\theta}}} \\
\alpha=4+\frac{22.14}{0.79+2.72 e^{47.63 \lambda_{\theta}}}
\end{gathered}
$$

The intermittency distributions based on the experimental results of the current study for a range of pressure gradients, free-stream turbulence levels and surface roughness conditions (Chapters 4,5 , and 6) have been found to be consistent with the dependence of $\alpha$ and $\sigma$ on only $\lambda_{\theta}$. This has been observed to be true in both attached flow and separated-flow transition. The remaining parameter required for estimating the intermittency distribution is the spot inception rate, $n$. This parameter has traditionally been extracted from the non-dimensional inception rate parameter, $N=\frac{n \sigma \theta_{t s}^{3}}{\nu}$ (Narasimha, 1957). The most recent model, proposed by Solomon et al. (1996), correlates this parameter to $\lambda_{\theta}$ and the free-stream turbulence intensity at the transition inception location $T u_{t s}$.

$$
\begin{array}{ll}
N=8.6 \times 10^{-4} e^{2.134 \lambda_{\theta_{t s}} \ln \left(T u_{t s}\right)-59.23 \lambda_{\theta_{t s}}-0.564 \ln \left(T u_{t s}\right)} & \left\{\lambda_{\theta t s} \leq 0\right\} \\
N=8.6 \times 10^{-4} e^{-0.564 \ln \left(T u_{t s}\right)-10 \sqrt{\lambda_{\theta_{t s}}}} & \left\{\lambda_{\theta t s}>0\right\}
\end{array}
$$




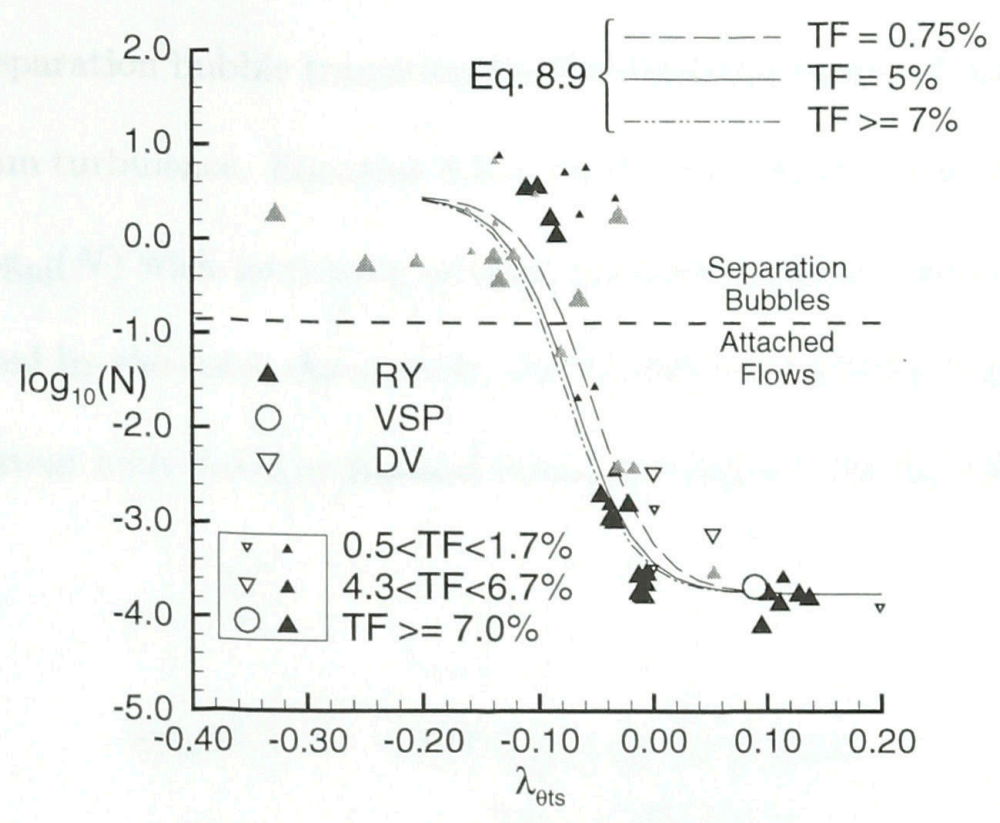

Figure 8.4: Variation of the spot inception parameter with $\lambda_{\theta_{t s}}$ and $T F$ (rough surface measurements are shown in grey)

However, as was discussed in Chapter 4, the accuracy of this model is sometimes inconsistent, and it is not intended to predict turbulent spot production rates in separation bubbles.

Figure 8.4 presents measured results for the variation of $N$ with $\lambda_{\theta}$ and free-stream turbulence level. The majority of the data points, as identified with filled symbols, are part of the present study for the $C_{P 1}, C_{P 2}$, and $C_{P 3}$ pressure distributions, and are observed to be consistent with the results of others. In the figure, the size of the symbols increases with increasing free-stream turbulence level. Unlike the earlier data sets used in the development of Eqs. 8.8-a and 8.8-b, the figure includes results from transition in separation bubbles. The attached and separated flow transition regimes are separated approximately by a straight dashed line. Despite 
somewhat larger scatter, the rate of spot production is observed to remain fairly constant in separation bubble transition for the displayed ranges of magnitudes of $\lambda_{\theta}$ and free-stream turbulence. Equation 8.8-a, on the other hand, would predict a linear increase in $\log _{10}(N)$ with increasing adverse pressure gradient, rather than levelling off as suggested by the data. As a result, the following correlation is proposed which is more consistent with the experimental results throughout the $\lambda_{\theta_{t s}}$ range of Fig. 8.4:

$$
\begin{aligned}
\log _{10}(N) & =-3.75+\frac{8.5}{2+35 e^{35\left(\lambda_{\theta}-f(T F)\right)}} \\
f(T F) & =\frac{2.9-0.25 T F(\%)}{100+1.4 T F(\%)(1+T F(\%))}
\end{aligned}
$$

This correlation is formulated such that it provides similar results to Eq. 8.8-a in the range of $\lambda_{\theta_{t s}}$ from -0.1 to 0.08 , for turbulence intensities varying from $0.5 \%$ to $6.5 \%$, since this earlier correlation was calibrated against an extensive data set in this range. The turbulence intensity, $T u$, appearing in the earlier expressions for $N$ is now replaced by $T F$. This was done in recognition of the fact that the effect of turbulence intensity on the transition process ought to be influenced by the length scale of the free-stream turbulence eddies. In the absence of length-scale information for free-stream turbulence, Eq. 8.9 may still be used to obtain estimates of $N$ by using $T u$ in place of $T F$. The data points shown with grey symbols in Fig. 8.4 correspond to a range of rough-surface conditions, and will be discussed shortly.

A more effective approach to estimating the spot production rate parameter, $N$, is evident from the results shown in Figure 8.5. Excluding the rough-surface 


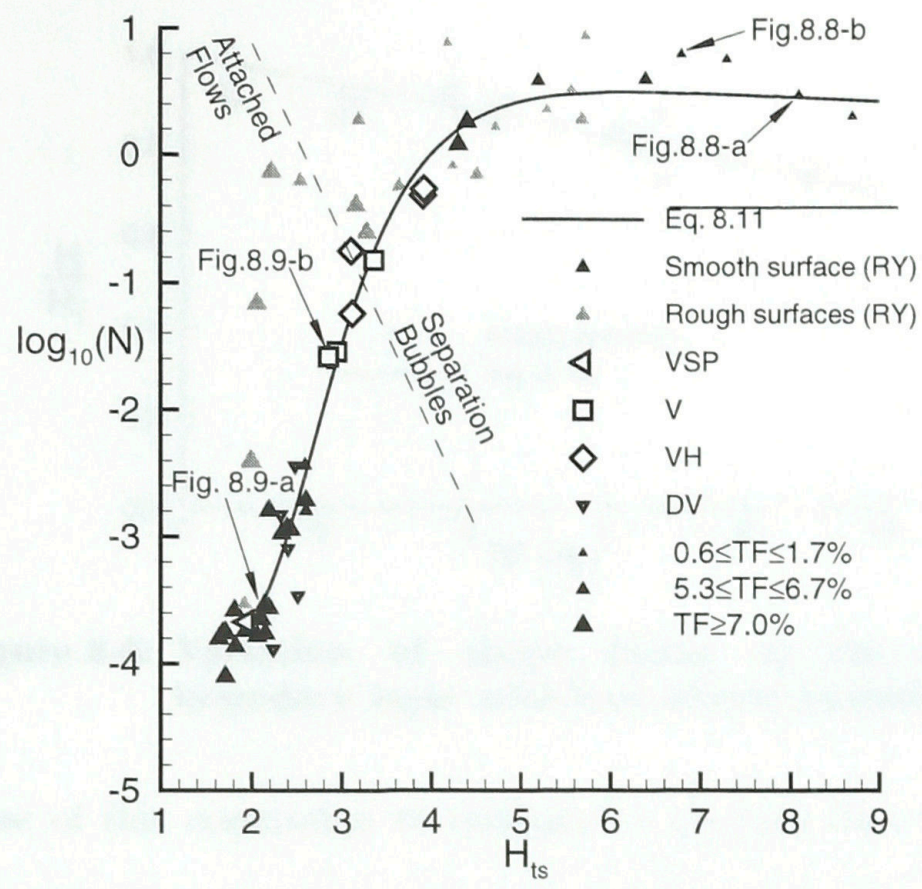

Figure 8.5: Variation of the spot production parameter with the shape factor at transition inception (rough surface measurements are shown in grey)

conditions identified by grey symbols, a strong dependence of $N$ on the boundary layer shape factor at the point of transition inception is observed. This is consistent with the established dependence of $N$ on the free-stream turbulence level and streamwise pressure gradient, for both factors are known to affect the shape factor. However, the notably lower scatter in Fig. 8.5 than that observed in Fig. 8.4 may suggest the boundary layer shape factor to be more directly relevant to the rate of turbulent spot production. The trends in Fig. 8.5 suggest the following correlation between $N$ and $H_{t s}:$

$$
\log _{10}(N)=\frac{0.55 H_{t s}-2.2}{1-0.63 H_{t s}+0.14 H_{t s}^{2}}
$$




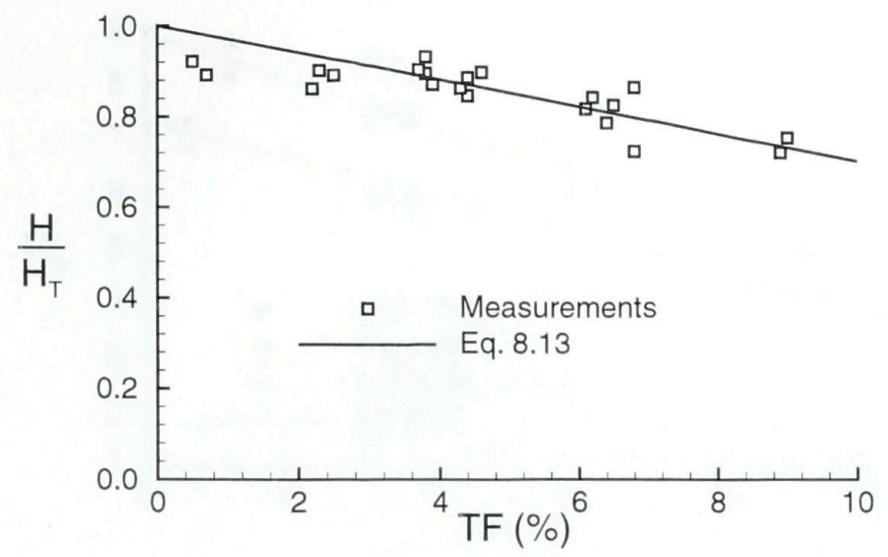

Figure 8.6: Variation of shape factor in the laminar boundary layer with free-stream turbulence

The use of this correlation to estimate $N$ requires that $H_{t s}$ be known. In instances of attached flow transition, this information can be obtained by the use of an integral method, such as the method of Thwaites (1949), for the development of the laminar boundary layer up to the point of transition inception. Based on the data published by White (1991), $H$ can be correlated to $\lambda_{\theta}$ through:

$$
H=\frac{2.61+20.3 \lambda_{\theta}}{1+9.43 \lambda_{\theta}+8.35 \lambda_{\theta}^{2}}
$$

The effect of free-stream turbulence on $H$ is not accounted for in this correlation, and can be estimated from the following expression, which is based on the current results, shown in Fig. 8.6.

$$
\frac{H}{H_{T}}=1-0.003 \operatorname{TF}(\%) \quad\{\text { TF }<11 \%\}
$$

where $H_{T}$ is the shape factor estimated from Eq. 8.12. 


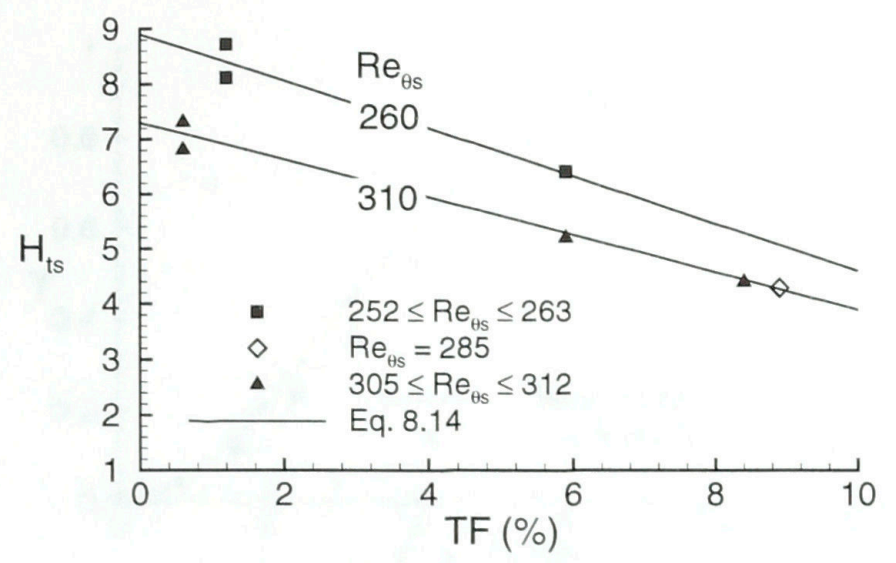

Figure 8.7: Variation of $H_{t s}$ with turbulence level and $R e_{\theta_{s}}$ for separation bubble transition (smooth surfaces)

For estimating the shape factor at the point of transition inception in separation bubbles, the following correlation is proposed for smooth surfaces, over the ranges of $250=R e_{\theta_{s}}=310$ and $T F \leq 10 \%$, based on the experimental data shown in Fig. 8.7.

$$
H_{t s}=\left(17.2-0.032 R e_{\theta_{s}}\right)-\left(0.9-0.0018 \operatorname{Re}_{\theta_{s}}\right) T F(\%)
$$

In summary, the proposed model for predicting the length of the transition zone, unified for attached and separated flow transition, consists of Eqs. 8.5, 8.7, 8.6, and 8.11. These are supplemented by Eq. 8.1 or 8.4 to predict the location of transition inception, and by Eqs. 8.12, 8.13, and 8.14 to predict the shape factor at this point, if necessary.

For two of the separated-flow transition cases labelled in Fig. 8.5, the predicted intermittency distribution, hence transition length, is compared to the measured 


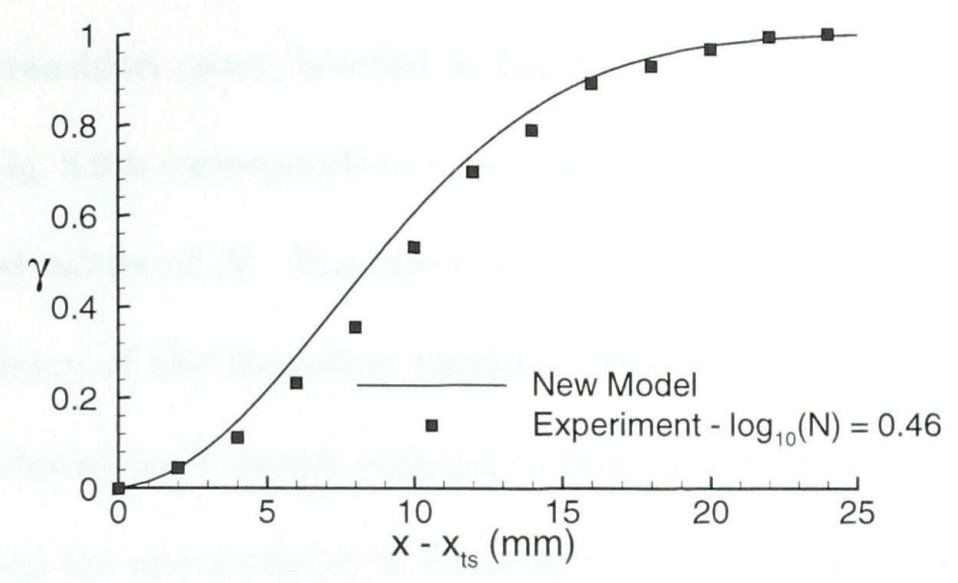

(a)

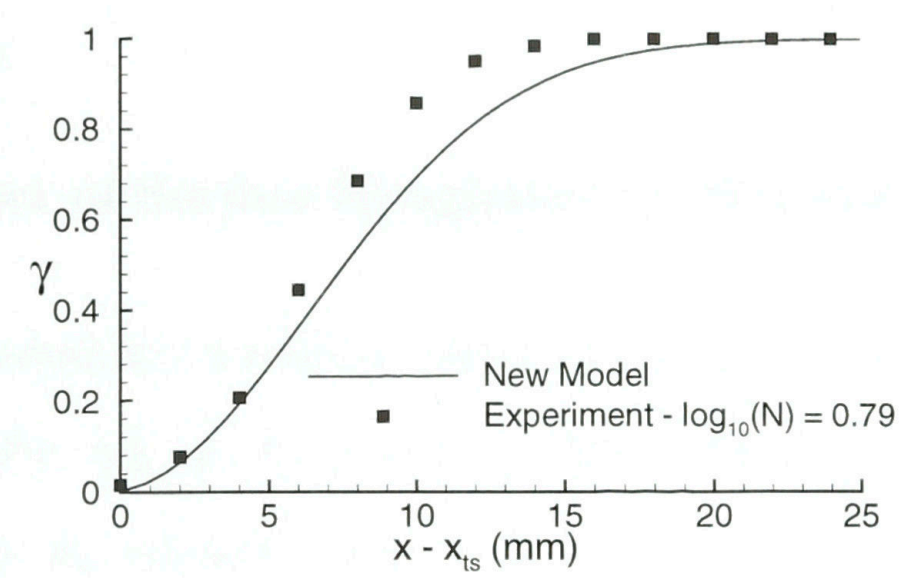

(b)

Figure 8.8: Intermittency distributions for two separated flow transition cases (as labeled in Fig. 8.5)

values in Figs. 8.8-a and 8.8-b. The spot production rate, $N$, of the test case corresponding to Fig. 8.8-b is off of the trend line (Eq. 8.11) in Fig. 8.5 by about one standard deviation. Thus, the difference in the extent of agreement of the predicted and experimental intermittency distributions in Figs. 8.8-a and 8.8-b provides an indication of the effect of the scatter in the measured values of $N$ on the prediction accuracy of the transition length. This effect is noted to be relatively small. 
Similarly, the measured and predicted intermittency distributions for two attached flow transition cases (labelled in Fig. 8.5) are compared in Figs. 8.9-a and 8.9-b. Again, Fig. 8.9-b corresponds to a test case that represents the extent of scatter in the measured values of $N$. The effect of the uncertainty in the $N$ values on the prediction accuracy of the transition length is observed to be small. For reference, the predictions based on $N$ values estimated using the original Solomon et al. (1996) model (Eq. 8.8-a) are also included in these figures. In general, the transition rate is predicted more accurately using the proposed spot production model (Eq. 8.11) than using Eq. 8.8-a.

\subsubsection{Effect of Surface Roughness on Transition Length}

For attached flow transition, surface roughness is noted to increase $N$ for a given $\lambda_{\theta_{t s}}$ (Fig. 8.4) or $H_{t s}$ (Fig. 8.5). This trend is particularly evident in Fig. 8.5, where $H_{t s}$ assumes a nearly constant value of 2 for the attached flow transition cases over rough surfaces. The rough surface data points shown in the figure for attached flow transition correspond to a range of roughness heights of about $0.1<k_{r m s} / \theta_{t s}<0.6$, with a trend toward increasing spot production rate with increasing roughness height. It therefore appears that while free-stream turbulence affects the spot production rate primarily through its influence on the shape factor, surface roughness has a more direct effect. Since turbulent spots are generated in the lower portion of the boundary layer (typically $0.15<y / \delta<0.25$ ), they can be more readily affected by the flow perturbations created by the roughness elements, compared to those generated in the free-stream. Further experiments are required 


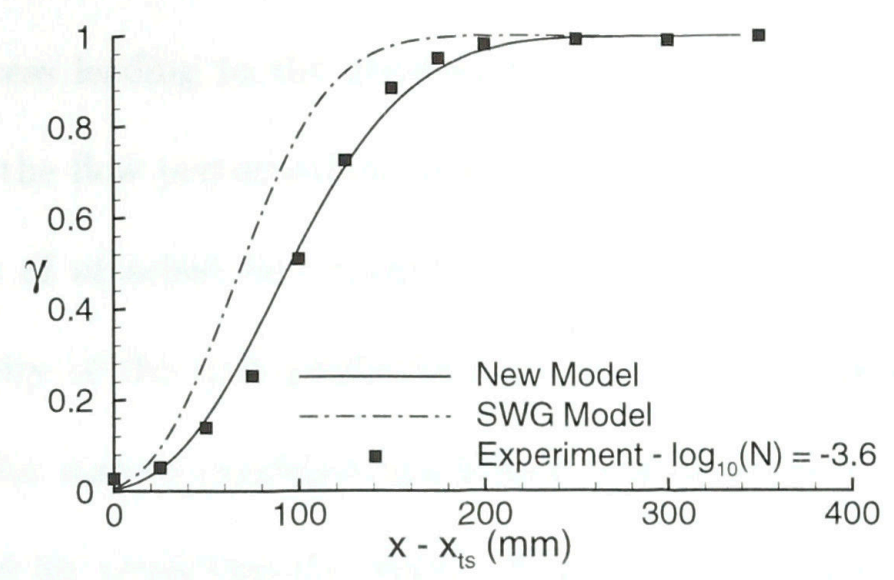

(a)

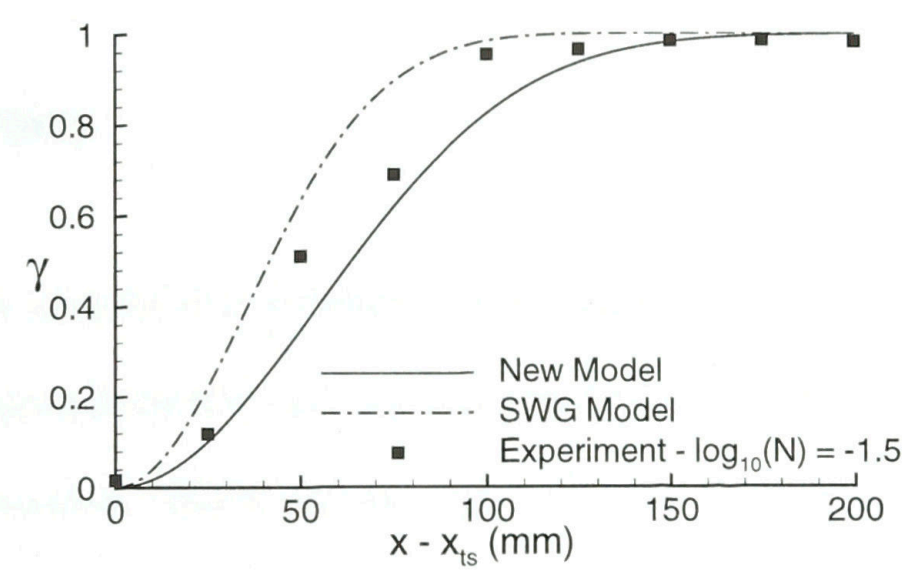

(b)

Figure 8.9: Intermittency distributions for two attached flow transition cases (as labeled in Fig. 8.5)

before an extension of the proposed correlation for $N$ may be attempted to include rough surface conditions in the attached flow transition regime.

Finally, as shown in Fig. 8.5, the spot production rate in instances of separation bubble transition does not appear to be sensitive to surface roughness, with the exception of two data points. In the majority of the separated flow transition cases shown in this figure, the separated shear layer was determined to be well above the 
crests of the roughness elements in the vicinity of the location of transition inception. Thus, the process leading to the generation of turbulent spots is not expected to be affected by the flow perturbations of the roughness elements to the same extent as in instances of attached flow transition. This may be the explanation for the lack of sensitivity of the spot production rate to surface roughness in these cases. Consequently, for surface roughness conditions typical of low pressure turbines, the proposed model for predicting the length of the transition zone remains applicable for the separated-flow regime.

\subsection{Summary}

Through analysis of an extensive experimental data set, a strong correlation of the turbulent spot production rate with the boundary layer shape factor at transition inception is identified. Based on this observation, a unified model is proposed for predicting the spot production rate in separated and attached flow regimes, for both favourable and adverse pressure gradients. The model is shown to maintain the prediction accuracy of an existing model for the attached flow regime, and is the first to allow prediction of the spot production rate in the separated regime. Additionally, in the separated flow transition regime, the model is shown to remain applicable for the majority of rough surface conditions typical of turbomachinery blades.

The model proposed for predicting the length of the transition zone is complemented by a model for locating the position of transition inception in separation bubbles. The proposed model provides improvements over existing 
alternatives both in terms of the precision of the predictions, and by accounting for the effects of free-stream turbulence and surface roughness. 


\section{Conclusions}

This thesis has presented measurements and simulations of the transition process in separation bubbles and attached flows, for a range of flow and surface conditions typical of low-pressure turbines of gas turbine engines. From these measurements, the relative importance to the transition process of flow Reynolds number, free-stream turbulence, periodic free-stream flow unsteadiness, roughness height, and roughness geometry has been established. The main conclusions of the study are summarized as follows:

- Variations in the free-stream turbulence level do not affect the transition rate in attached flows, while the transition rate in separation bubbles decreases slightly with increased turbulence level. For both attached and separated flows, the transition rate is not directly affected by the flow Reynolds number.

- The time-averaged locations of flow separation, transition inception, and reattachment are insensitive to periodic free-stream velocity oscillations, for the range of frequencies and amplitudes considered. This conclusion persists for all values of free-stream turbulence and flow Reynolds number considered in 
the study.

- Under periodic-unsteady free-stream conditions, the maximum bubble thickness is observed at the point of minimum free-stream velocity, which is the result expected based on a quasi-steady flow assumption. However, unsteady movement of the reattachment region is not quasi-steady, with the maximum bubble length observed at the point of maximum free-stream velocity.

- For conditions typical of low pressure turbine blades, surface roughness and freestream turbulence are found to be of equal importance in locating transition onset in separation bubbles. Additionally, the transition inception location is more sensitive to surface roughness at elevated free-stream turbulence levels.

- In the majority of separation bubble test cases, the rate of transition is not affected by the surface roughness. An increase in transition rate is observed in attached flows, and when the roughness peaks protrude significantly into the separated shear layer between separation and transition inception.

- In the majority of separation bubble experiments, the turbulent bursts in the separated shear layer were consistent with the hot-wire signals in attached flows. This provides evidence that transition occurred due to the viscous TollmienSchlichting instability in these instances.

- A reduction in the adverse pressure gradient resulted in a greater likelihood of separation bubble transition occurring via the inviscid Kelvin-Helmholtz mechanism, characterized by nearly instantaneous transition in the shear layer. 
This process is accompanied by the regular shedding of vortices from the bubble.

- Large-eddy simulation has been successfully used to model the transition process in a separation bubble. In the simulated test case, the Kelvin-Helmholtz instability is shown to be responsible for breakdown in the shear layer. However, the dominant frequency of vortex shedding matches that of the TollmienSchlichting waves observed upstream, demonstrating an interaction between these instability mechanisms.

- Finally, an improved transition model has been developed for use in engineering calculations. This model is the first to predict turbulent spot production rates in separation bubbles, and is also the first to predict the combined effects of surface roughness and free-stream turbulence on transition inception in separation bubbles. The accuracy of the model is demonstrated for a broad range of Reynolds number, pressure distributions, free-stream turbulence levels, and surface conditions. 


\section{References}

Abu-Ghannam, B. J. and Shaw, R., 1980, "Natural Transition of Boundary Layers

- The Effects of Turbulence, Pressure Gradient, and Flow History," J. Mech. Eng. Sci., 22, pp. 213-228.

Addison, J. S. and Hodson, H. P., 1990-a, "Unsteady Transition in an Axial Flow Turbine: Part 1 - Measurements on the Turbine Rotor," ASME J. Turbomach., 112, pp. 206-214.

Addison, J. S. and Hodson, H. P., 1990-b, "Unsteady Transition in an Axial Flow Turbine: Part 2 - Cascade Measurements and Modeling," ASME J. Turbomach., 112, pp. 215-221.

Alam, M. and Sandham, N. D., 2000, "Direct Numerical Simulation of 'Short' Laminar Separation Bubbles With Turbulent Reattachment," J. Fluid Mech., 410, pp. 1-28.

Anthony, R. J., Jones, T. V., and LaGraff, J., 2004, "High Frequency Surface Heat Flux Imaging of Bypass Transition," ASME Paper no. GT2004-54162.

Bao, F. and Dallmann, U. C., 2004, "Some Physical Aspects of Separation Bubble on a Rounded Backward-Facing Step," Aero. Sci. \& Tech., 8, pp. 83-91.

Belnap, B. J., van Rij, J. A., and Ligrani, P. M., 2002, "A Reynolds Analogy for Real Component Surface Roughness," Int. J. Heat \& Mass Transfer, 45, pp. 3089-3099. 
Bernal, L. P. and Roshko, A., 1986, "Streamwise Vortex Structure in Plane Mixing Layers," J. Fluid Mech., 170, pp. 499-525.

Blair, M. F., 1982, "Influence of Free-Stream Turbulence on Boundary Layer Transition in Favorable Pressure Gradients," ASME J. Eng. Power, 104, pp. 743750 .

Blair, M. F., 1994, "An Experimental Study of Heat Transfer in a Large Scale Turbine Rotor Passage," ASME J. Turbomach., 116, pp. 1-13.

Bons, J. P., 2002, "St and $c_{f}$ Augmentation for Real Turbine Roughness with Elevated Freestream Turbulence," ASME Paper no. GT2002-30198.

Bons, J. P. and McClain, S. T., 2003, "The Effect of Real Turbine Roughness with Pressure Gradient on Heat Transfer," ASME J. Turbomach., 126, pp. 385-394.

Bons, J. P., McClain, S. T., Taylor, R. P., and Rivir, R. B., 2001, "The Many Faces of Turbine Surface Roughness," ASME J. Turbomach., 123, pp. 739-748.

Boyle, R. J. and Senyitko, R. G., 2003, "Measurements and Predictions of Surface Roughness Effects on the Turbine Vane Aerodynamics," ASME Paper no. GT200338580 .

Boynton, J. L., Tabibzadeh, R., and Hudson, S. T., 1992, "Investigation of Rotor Blade Roughness Effects on Turbine Performance," ASME J. Turbomach., 115, pp. $614-620$.

Brendel, M. and Mueller, T. J., 1987, "Boundary Layer Measurements on an Airfoil at Low Reynolds Numbers," AIAA Paper no. 87-0495.

Butler, K. M. and Farrell, B. F., 1992, "Three-Dimensional Optimal Perturbations in Viscous Shear Flow," Phys. Fluids-A, 4, p. 1637.

Cabot, W., 1998, "Large Eddy Simulation of a Separated Boundary Layer," in: Annual Research Briefs - 1998, Center for Turbulence Research, Stanford University / NASA Ames, Stanford, CA. 
Chandrasekhar, S., 1961, Hydrodynamic and Hydromagnetic Stability, Clarendon Press, Oxford.

Chen, K. K. and Thyson, N. A., 1971, "Extension of Emmons' Spot Theory to Flows on Blunt Bodies," AIAA J., 9, pp. 821-825.

Chong, T. P. and Zhong, S., 2003, "On the Three-Dimensional Structure of Turbulent Spots," ASME Paper no. GT2003-38435.

Cummings, M. J. and Bragg, M. B., 1996, "Boundary-Layer Transition Due to Isolated Three-Dimensional Roughness on Airfoil Leading Edge," AIAA J., 34, pp. 1949-1952.

Davis, M. R., 1980, "Design of Flat Plate Leading Edges to Avoid Flow Separation," AIAA J., 18, pp. 598-600.

Davis, R. L., Carter, J. E., and Reshotko, E., 1987, "Analysis of Transitional Separation bubbles on Infinite Swept Wings," AIAA J., 25, pp. 421-428.

Devasia, K., 1974, A Study of the Effect of Pressure Gradients on Transition in the Boundary Layer on a Flat Plate, Master's thesis, Dept. Aeronaut. Eng., Indian Inst. Sci., Bangalore, India.

Dey, J. and Narasimha, R., 1988, "An Integral Method for the Calculation of 2-D Transitional Boundary Layers," Report 88-FM-7, Indian Inst. of Sci., Bangalore, India.

Dirling, R. B. J., 1973, "A Method for Computing Rough Wall Heat Transfer Rates on Reentry Nosetips," AIAA Paper no. 73-763.

Dong, Y. and Cumpsty, N. A., 1990-a, "Compressor Blade Boundary Layers: Part 1 - Test Faciltiy and Measurements with No Incident Wakes," ASME J. Turbomach., 112, pp. 222-230.

Dong, Y. and Cumpsty, N. A., 1990-b, "Compressor Blade Boundary Layers: Part 2 - Measurements with Incident Wakes," ASME J. Turbomach., 112, pp. 231-240. 
D'Ovidio, A., Harkins, J. A., and Gostelow, J. P., 2001, "Turbulent Spots in Strong Adverse Pressure Gradients - Part 2: Spot Propagation and Spreading Rates," ASME Paper no. 2001-GT-0406.

Dubois, T. and Bouchon, F., 1998, "Subgrid-Scale Models Based on Incremental Unknowns for Large Eddy Simulation," in: Annual Research Briefs - 1998, Center for Turbulence Research, Stanford University / NASA Ames, Stanford, CA, pp. $221-236$.

Ducros, F., Comte, P., and Lesieur, M., 1996, "Large-Eddy Simulation of Transition to Turbulence in a Boundary Layer Developing Spatially Over a Flat Plate," J. Fluid Mech., 326, pp. 1-36.

Dvorak, F. A., 1969, "Calculation of Turbulent Boundary Layers on Rough Surfaces in Pressure Gradient," AIAA J., 7, pp. 1752-1759.

Emmons, H. W., 1951, "The Laminar-Turbulent Transition in a Boundary Layer Part 1," J. Aeronaut. Sci., 18, pp. 490-498.

Estevadoral, J. and Kleis, S. J., 1999, "High-Resolution Measurements of TwoDimensional Instabilities and Turbulence Transition in Plane Mixing Layers," Exp. Fluids, 27, pp. 378-390.

Fraser, C. J., Higazy, M. G., and Milne, J. S., 1994, "End-Stage Boundary Layer Transition Models for Engineering Calculations," Proc. Inst. Mech. Eng., Part C: J. Mech. Eng. Sci., 208, pp. 47-58.

Frenkiel, F. N., 1948, "The Decay of Isotropic Turbulence," J. Appl. Mech., pp. $311-321$.

Fringer, O. B., 2004. http://fluid.stanford.edu/ fringer/movies/shear_convect/kh.html.

Gaster, R., 1967, "The Structure and Behavior of Laminar Separation-Bubbles," ARC R\&M 3595. 
Gibbings, J. C. and Al-Shukri, S. M., 1997, "Effect of Sandpaper Roughness and Stream Turbulence on the Laminar Layer and its Transition," Aeronaut. J., 101, pp. 17-24.

Gibbings, J. C., Goksel, O. T., and Hall, D. J., 1986-a, "The Influence of Roughness Trips Upon Boundary-layer Transition - Part 1 Characterstics of Wire Trips," Aeronaut. J., 90, pp. 289-301.

Gibbings, J. C., Goksel, O. T., and Hall, D. J., 1986-b, "The Influence of Roughness Trips upon Boundary-layer Transition - Part 2 Characteristics of Single Spherical Trips," Aeronaut. J., 90, pp. 357-367.

Gibbings, J. C., Goksel, O. T., and Hall, D. J., 1986-c, "The Influence of Roughness Trips upon Boundary-layer Transition - Part 3 Characteristics of Rows of Spherical Transition Strips," Aeronaut. J., 90, pp. 393-398.

Goldstein, M. E., 1985, "Scattering of Acoustic Waves into Tollmien-Schlichting Waves by Small Streamwise Variations in Surface Geometry," J. Fluid Mech., 154, pp. 509-529.

Görtler, H., 1940, "Uber ein Dreidimensionale Instabilitat Laminarer Grenzshichten an Konkaven Wanden," Math. Phys. K1.2:1; also NACA TM-1375 (1954) (in English).

Gostelow, J. P. and Blunden, A. R., 1989, "Investigations of Boundary Layer Transition in an Adverse Pressure Gradient," ASME J. Turbomach., 111, pp. $366-375$.

Gostelow, J. P., Blunden, A. R., and Walker, G. J., 1994, "Effects of Free-Stream Turbulence and Adverse Pressure Gradient on Boundary Layer Transition," ASME J. Turbomach., 116, pp. 392-404.

Gostelow, J. P., Hong, G., Melwani, N., and Walker, G. J., 1993, "Turbulent Spot Developement under a Moderate Adverse Pressure Gradient," ASME 93-GT-377. 
Gostelow, J. P., Melwani, N., and Walker, G. J., 1996, "Effects of Streamwise Pressure Gradient on Turbulent Spot Development," ASME J. Turbomach., 118, pp. 737743.

Gostelow, J. P. and Thomas, R. L., 2005, "Response of a Laminar Separation Bubble to an Impinging Wake," ASME J. Turbomach., 127, pp. 35-42.

Hall, A. A. and Hislop, G. S., 1938, "Experiments on the Transition of the Laminar Boundary Layer on a Flat Plate," ARC R \& M 1843.

Ham, F. E., Lien, F. S., Wu, X., Wang, M., and Durbin, P., 2000, "LES and Unsteady RANS of Boundary Layer Transition Induced by Periodically Passing Wakes," in: Proceedings of the Summer Program 2000, Center for Turbulence Research, Stanford University, Stanford, CA.

Harrar, D. L. and Osborne, M. R., 2003, "Computing Eigenvalues of Ordinary Differential Equations," ANZIAM J., 44(E), pp. C313-C334.

Hatman, A. and Wang, T., 1998, "Separated-Flow Transition Part 3 - Primary Modes and Vortex Dynamics," ASME Paper no. 98-GT-463.

Hatman, A. and Wang, T., 1999, "A Prediction Model for Separated Flow Transition," ASME J. Turbomach., 121, pp. 594-602.

Haueisen, V., Henneke, D. K., and Schröder, T., 1997, "Measurements with Surface Mounted Hot Film Sensors on Boundary Layer Transition in Wake Disturbed Flow," AGARD CP-598.

Hobson, G. V. and Weber, S., 2000, "Prediction of a Laminar Separation Bubble over a Controlled-Diffusion Compressor Blade," ASME Paper no. 2000-GT-277.

Hodson, H. P., 1991, "Aspects of Unsteady Blade-Surface Boundary Layers and Transition in Axial Turbomachines," in: VKI Lecture Series on Boundary Layers in Turbomachines, von Kármán Institute for Fluid Dynamics, Rhode-Saint-Genese, Belgium. 
Horton, H. P., 1968, "A Semi-Empirical Theory for the Growth and Bursting of Laminar Separation Bubbles," ARC CP-1073.

Hourmouziadis, J., 1989, "Aerodynamic Design of Low Pressure Turbines," AGARD Lecture Series no. 167.

Huai, X., Joslin, R. D., and Piomelli, U., 1997, "Large-Eddy Simulation of Transition to Turbulence in Boundary Layers," Theor. Comput. Fluid Dyn., 9, pp. 149-163.

Johnson, M. W., 1994, "A Bypass Transition Model for Boundary Layers," ASME J. Turbomach., 116, pp. 759-764.

Johnson, M. W., 1998, "Turbulent Spot Characteristics in Boundary Layers Subjected to Streamwise Pressure Gradient," ASME Paper no. 98-GT-124.

Johnson, M. W., 2003, "A Receptivity Based Transition Model," ASME Paper no. GT2003-38073.

Johnson, M. W. and Dris, A., 2000, "The Origin of Turbulent Spots," ASME J. Turbomach., 122, pp. 88-92.

Johnson, M. W. and Ercan, A. H., 1997, "Predicting Bypass Transition: A Physical Model versus Empirical Correlations," ASME Paper no. 97-GT-475.

Johnson, M. W. and Fashifar, A., 1994, "Statistical Properties of Turbulent Bursts in Transitional Boundary Layers," Int. J. Heat \& Fluid Flow, 5, pp. 283-290.

Kachanov, Y. S., 2000, "Three-Dimensional Receptivity of Boundary Layers," Eur. J. Mech. B/Fluids, 19, pp. 723-744.

Kaszeta, R. W., Simon, T. W., and Ashpis, D. P., 2001, "Experimental Investigation of Transition to Turbulence as Affected by Passing Wakes," ASME Paper no. 2001GT-0195.

Katz, Y., Seifert, A., and Wyganski, I., 1990, "On the Evolution of the Turbulent Spot in a Laminar Boundary Layer with a Favourable Pressure Gradient," J. Fluid Mech., 221, pp. 1-22. 
Kerho, M. F. and Bragg, M. B., 1997, "Airfoil Boundary-Layer Development and Transition with Large Leading-Edge Roughness," AIAA J., 35, pp. 75-84.

Kim, J., Simon, T. W., and Russ, S. G., 1992, "Free-Stream Turbulence and Concave Curvature Effects on Heated, Transitional Boundary Layers," ASME J. Heat Transfer, 114, pp. 338-347.

Kind, R. J., Serjak, P. J., and Abbott, M. W. P., 1998, "Measurements and Prediction of the Effects of Surface Roughness on Profile Losses and Deviation in a Turbine Cascade," ASME J. Turbomach., 120, pp. 20-27.

Klebanoff, P. S. and Tidstrom, K. D., 1982, "Mechanism by Which a TwoDimensional Roughness Element Induces Boundary-Layer Transition," Phys. Fluids, 15, pp. 1173-1188.

Klebanoff, P. S., Tidstrom, K. D., and Sargent, L. M., 1961, "The Three-dimensional Nature of Boundary-layer Transition," J. Fluid Mech., 12, pp. 1-42.

Lang, H., Mørck, T., and Woisetschläger, J., 2002, "Stereoscopic Particle Image Velocimetry in a Transonic Turbine Stage," Exp. Fluids, 32, pp. 700-709.

Lang, M., Rist, U., and Wagner, S., 2004, "Investigations on Controlled Transition Development in a Laminar Separation Bubble by Means of LDA and PIV," Exp. Fluids, 36, pp. 43-52.

Leipold, R., Boese, M., and Fottner, L., 2000, "The Influence of Technical Surface Roughness Caused by Precision Forging on the Flow Around a Highly Loaded Compressor Cascade," ASME J. Turbomach., 122, pp. 416-425.

Leonard, A., 1996, "Direct Numerical Simulation of Turbulent Flows," in: Simulation and Modeling of Turbulent Flows, T. Gatski, M. Hussaini, and J. Lumley, eds., Oxford University Press, New York.

Lesieur, M. and Métais, O., 1996, "New Trends in Large-Eddy Simulations of Turbulence," Annu. Rev. Fluid Mech., 28, pp. 45-82. 
Lou, W. and Hourmouziadis, J., 2000, "Separation Bubbles Under Steady and Periodic-Unsteady Main Flow Conditions," ASME J. Turbomach., 122, pp. 634643.

Mack, L. M., 1984, "Boundary Layer Linear Stability Theory," AGARD RN709.

Malkiel, E. and Mayle, R. E., 1996, "Transition in a Separation Bubble," ASME J. Turbomach., 118, pp. 752-759.

Martin, B. W., Brown, A., and Garrett, S. E., 1978, "Heat Transfer to a PVD Rotor at High Subsonic Passage Throat Mach Number," Proc. Inst. Mech. Eng., 192, pp. 225-235.

Maucher, U., Rist, U., Kloker, M., and Wagner, S., 1999, "DNS of Laminar-Turbulent Transition in Separation-Bubbles," in: High Performance Computing in Science and Engineering '99, E. Krause, and W. Jäger, eds., Springer, New York, pp. 279294.

Mayle, R. E., 1991, "The Role of Laminar-Turbulent Transition in Gas Turbine Engine," ASME J. Turbomach., 113, pp. 509-537.

Mellen, C. P., Frölich, J., and Rodi, W., 2002, "Lessons from the European LESFOIL Project on LES of Flow around an Airfoil," AIAA Paper no. 2002-0111.

Menter, F. R., Langtry, R. B., Likki, S. R., Suzen, Y. B., Huang, P. G., and Völker, S., 2004, "A Correlation-Based Transition Model Using Local Variables: Part I Model Formulation," ASME Paper no. GT2004-53452.

Mills, R., Sheridan, J., and Hourigan, K., 2003, "Particle Image Velocimetry and Visualization of Natural and Forced Flow Around Rectangular Cylinders," J. Fluid Mech., 478, pp. 299-323.

Morkovin, M. V., 1969, "On the Many Faces of Transition," in: Viscous Drag Reduction, C. S. Wells, ed., Plenum Press, New York, pp. 1-31. 
Morris, H. M., 1955, "A New Concept of Flow in Rough Conduits," Transactions ASCE, 120, pp. 373-398.

Moser, R. D. and Rogers, M. M., 1993, "Three-Dimensional Evolution of a Plane Mixing Layer: Pairing and Transition to Turbulence," J. Fluid Mech., 247, pp. 275-320.

Müller, M., Gallus, H. E., and Niehuis, R., 2000, "A Study on Models to Simulate Boundary Layer Transition in Turbomachinery Flows," ASME Paper no. 2000GT-274.

Narasimha, R., 1957, "On the Distribution of Intermittency in the Transition Region of a Boundary Layer," J. Aeronaut. Sci., 24, pp. 711-712.

Narasimha, R., 1985, "The Laminar-Turbulent Transition Zone in the Boundary Layer," Prog. Aero. Sci., 22, pp. 29-80.

Passchier, D. M., 1997, "Hot-Wire Measurements in the Neighbourhood of a Laminar Separation-Bubble," in: Boundary Layer Separation in Aircraft Dynamics, R. Henkes, and D. Bakker, eds., Delft University Press, Delft, NL, pp. 69-86.

Pfeil, H., Herbst, R., and Schröder, T., 1983, "Investigation of the Laminar-Turbulent Transition of Boundary Layers Disturbed by Wakes," ASME J. Eng. Power, 97, pp. 261-274.

Pinson, M. W. and Wang, T., 1997, "Effects of Leading-edge Roughness on Fluid Flow and Heat Transfer in the Transitional Boundary Layer over a Flat Plate," Int. J. Heat \& Mass Transfer, 40, pp. 2813-2823.

Piomelli, U. and Balaras, E., 2002, "Wall-Layer Models for Large Eddy Simulations," Annu. Rev. Fluid Mech., 34, pp. 349-374.

Pope, S. B., 2000, Turbulent Flows, Cambridge University Press, New York.

Pullan, G., 2004, "Secondary Flows and Loss Caused by Blade Row Interaction in a Turbine Stage," ASME 2004GT-53743. 
Rai, M. M. and Moin, P., 1993, "Direct Numerical Simulation of Transition and Turbulence in a Spatially Evolving Boundary Layer," J. Comput. Phys., 109, pp. 169-192.

Reshotko, E., 1976, "Boundary Layer Stability and Transition," Annu. Rev. Fluid Mech., 8, pp. 311-347.

Reshotko, E., 2001, "Transient Growth: A Factor in Bypass Transition," Phys. Fluids, 13, pp. 1067-1075.

Reynolds, O., 1883, "An Experimental Investigation of the Circumstances which Determine Whether the Motion of Water Shall Be Direct or Sinuous and of the Law of Resistance in Parallel Channel," Philo. Trans. of the Royal Soc., 174, pp. 935-982.

Rist, U. and Maucher, U., 2002, "Investigations of Time-Growing Instabilities in Laminar Separation Bubbles," Eur. J. Mech. B/Fluids, 21, pp. 495-509.

Roach, P. E., 1997, "The Generation of Nearly Isotropic Turbulence by Means of Grids," Int. J. Heat \& Fluid Flow, 8, pp. 82-92.

Roberts, W. B., 1975, "The Effect of Reynolds Number and Laminar Separation on Axial Cascade Performance," ASME J. Eng. Power, 97, pp. 261-274.

Roberts, W. B., 1980, "Calculation of Laminar Separation Bubbles and Their Effect on Airfoil Performance," AIAA J., 18, pp. 25-31.

Rodi, W., Ferziger, J. H., Breuer, M., and Pourquié, M., 1997, "Status of Large Eddy Simulation: Results of a Workshop," J. Fluids Eng., 119, pp. 248-262.

Rodi, W. and Sheuerer, G., 1985, "Calculation of Laminar-Turbulent Boundary Layer Transition on Turbine Blades," in: AGARD Conference Proceedings No. 390, pp. 181-18.13.

Ruban, A. I., 1985, "On the Generation of Tollmien-Schlichting Waves by Sound," Fluid Dynamics, 19, pp. 709-716. 
Sankaran, M., Chambers, A. J., and Antonia, R. A., 1986, "The Influence of A Favourable Pressure Gradient on the Growth of a Turbulent Spot," in: Proc. 9th Australasian Fluid Mech. Conf., Auckland, NZ, pp. 342-345.

Sanz, W. and Platzer, M. F., 1997, "On the Calculation of Transitional Separation Bubbles Using Different Models," ASME Paper no. 97-GT-453.

Savill, A. M., 1992, "Evaluating Turbulence Model Predictions of Transition - an ERCOFTAC SIG Project," Appl. Sci. Res., 51, p. 555.

Schlichting, H. and Gersten, K., 2000, Boundary Layer Theory, Springer-Verlag, Berlin.

Schmid, P. J. and Henningson, D. S., 2001, Stability and Transition in Shear Flows, Springer, New York.

Schmidt, R. C. and Patankar, S. V., 1991, "Simulating Boundary Layer Transition With Low-Reynolds-Number $k-\epsilon$ Turbulence Models: Part 1 - An Evaluation of Prediction Characteristics," ASME J. Turbomach., 113, pp. 10-17.

Schobeiri, M. T. and Radke, R. E., 1994, "Effects of Periodic Unsteady Wake Flow and Pressure Gradient on Boundary Layer Transition Along the Concave Surface of a Curved Plate," ASME Paper no. 94-GT-327.

Schobeiri, M. T., Read, K., and Lewalle, J., 1995, "Effect of Unsteady Wake Passing Frequency on Boundary Layer Transition, Experimental Investigation and Wavelet Analysis," ASME Paper no. 95-GT-437.

Schubauer, G. B. and Klebanoff, P. S., 1955, "Contributions on the Mechanics of Boundary-layer Transition," NACA TN 3489.

Schubauer, G. B. and Skramstad, H. K., 1948, "Laminar Boundary-Layer Oscillations and Transition on a Flat Plate," NACA TN 909.

Schultz, M. P. and Volino, R. J., 2001, "Effects of Concave Curvature on Boundary Layer Transition under High Free-Stream Turbulence Conditions," ASME Paper no. 2001-GT-0191. 
Sharma, O. P., Renaud, E., Butler, T. L., Milsaps, K. J., Dring, R. P., and Joslyn, H. D., 1988, "Rotor-Stator Interaction in Multi-Stage Axial-Flow Turbines," AIAA Paper no. 88-3013.

Sigal, A. and Danberg, J. E., 1990, "New Correlation of the Roughness Density Effect on the Turbulent Boundary Layer," AIAA J., 20, pp. 554-556.

Singer, B. A. and Joslin, R. D., 1994, "Metamorphosis of a Hairpin Vortex into a Young Turbulent Spot," Phys. Fluids, 6, pp. 3724-3736.

Solomon, W. J. and Walker, G. J., 1995, "Observations of Wake Induced Transition on an Axial Compressor Blade," ASME Paper no. 95-GT-381.

Solomon, W. J., Walker, G. J., and Gostelow, J. P., 1996, "Transition Length Prediction for Flows with Rapidly Changing Pressure Gradients," ASME J. Turbomach., 118, pp. 744-751.

Spalart, P. R., 2000, "Strategies for Turbulence Modeling and Simulations," Int. J. Heat \& Fluid Flow, 21, pp. 252-263.

Spalart, P. R. and Allmaras, S. R., 1992, "A One-Equation Turbulence Model for Aerodynamic Flows," AIAA Paper no. 92-0439.

Spalart, P. R. and Strelets, M. K., 2000, "Mechanisms of Transition and Heat Transfer in a Separation Bubble," J. Fluid Mech., 403, pp. 329-349.

Steelant, J. and Dick, E., 1996, "Modeling of Bypass Transition with Conditioned Navier-Stokes Equations Coupled to an Intermittency Transport Equation," Int. J. Numer. Methods Fluids, 23, pp. 193-220.

Stieger, R. D. and Hodson, H. P., 2004, "The Transition Mechanism of Highly-Loaded LP Turbine Blades," ASME J. Turbomach., 126, pp. 536-543.

Stripf, M., Schulz, A., and Wittig, S., 2005, "Surface Roughness Effects on External Heat Transfer of a HP Turbine Vane," ASME J. Turbomach., 127, pp. 200-208. 
Suder, K. L., Chima, R. V., Strazisar, A. J., and Roberts, W. B., 1995, "The Effect of Adding Roughness and Thickness to a Transonic Axial Compressor Rotor," ASME J. Turbomach., 117, pp. 491-505.

Suzen, Y. B. and Huang, P. G., 2000, "Modelling of Flow Transition Using an Intermittency Transport Equation," J. Fluids Eng., 122, pp. 273-284.

Taylor, G. I., 1939, "Some Recent Developments in the Study of Turbulence," in: Proc. 5th Intl. Cong. Appl. Mech, J. P. Den Hartog, and H. Peters, eds., Wiley, New York, pp. 294-310.

Taylor, R. P., 1990, "Surface Roughness Measurements on Gas Turbine Blades," ASME J. Turbomach., 112, pp. 175-180.

Thwaites, B., 1949, "Approximate Calculation of the Laminar Boundary Layer," Aeronaut. Q., 7, pp. 245-280.

Thwaites, B., (ed.) , 1960, Incompressible Aerodynamics, Oxford University Press, Oxford, UK.

Tollmien, W., 1929, "Über die Entstehung der Turbulenz," Nachr. Ges. Wiss. Göttingen Math.-Phys. Kl. 1929, pp. 21-44.

Turner, A. B., 1971, "Local Heat Transfer Measurements on a Gas Turbine Blade," J. Mech. Eng. Sci., 13, pp. 1-12.

van Hest, B. F. A., Pesschier, D. M., and van Ingen, J. L., 1994, "The Development of a Turbulent Spot in an Adverse Pressure Gradient Boundary Layer," in: Proc. IUTAM Symposium, Sendai, JP.

Volino, R. J., 2002-a, "An Investigation of the Scales in Transitional Boundary Layers Under High Free-Stream Turbulence Conditions," ASME Paper no. GT2002-30233.

Volino, R. J., 2002-b, "Separated Flow Transition under Simulated Low-Pressure Turbine Airfoil Conditions: Part 1 - Mean Flow and Turbulence Statistics," ASME J. Turbomach., 124, pp. 645-655. 
Volino, R. J., 2002-c, "Separated Flow Transition under Simulated Low-Pressure Turbine Airfoil Conditions: Part 2 - Turbulence Spectra," ASME J. Turbomach., 124, pp. 656-664.

Volino, R. J. and Bohl, D. G., 2004, "Separated Flow Transition Mechanism and Prediction with High and Low Freestream Turbulence under Low Pressure Turbine Conditions," ASME Paper no. GT2004-53360.

Volino, R. J. and Hultgren, L. S., 2001, "Measurements in Separated and Transitional Boundary Layers Under Low-Pressure Turine Airfoil Conditions," ASME J. Turbomach., 123, pp. 189-197.

Volino, R. J., Schultz, M. P., and Pratt, C. M., 2001, "Conditional Sampling in a Transitional Boundary Layer under High Freestream Turbulence Conditions," ASME Paper no. 2001-GT-0192.

Volino, R. J. and Simon, T. W., 1995, "Bypass Transition in Boundary Layers Including Curvature and Favorable Pressure Gradient Effects," ASME J. Turbomach., 117, pp. 166-174.

Waigh, D. R. and Kind, R. J., 1998, "Improved Aerodynamic Characterization of Regular Three-Dimensional Roughness," AIAA J., 36, pp. 1117-1119.

Walker, G. J., 1989, "Transitional Flow on Axial Compressor Blading," AIAA J., 27, pp. 595-602.

Walker, G. J., Subroto, P. H., and Platzer, M. F., 1988, "Inviscid Interaction Analysis of Low Reynolds Number Airfoil Flows Involving Laminar Separation Bubbles," ASME Paper no. 88-GT-32.

Wang, T. and Rice, M. C., 2003, "Effect of Elevated Free-Stream Turbulence on Transitional Heat Transfer over Dual-Scaled Rough Surfaces," ASME Paper no. GT2003-38835. 
Wang, T. and Simon, T. W., 1987, "Heat Transfer and Fluid Mechanics Measurements in a Boundary Layer Undergoing Transition on a Convex-Curved Wall," ASME J. Turbomach., 109, pp. 443-452.

Watmuff, J. H., 1999, "Evolution of a Wave Packet into Vortex Loops in a Laminar Separation Bubble," J. Fluid Mech., 397, pp. 119-139.

White, F. M., 1991, Viscous Fluid Flow, McGraw-Hill, New York, 2 ed.

Wilcox, D. C., 1977, "A Model for Transitional Flows," AIAA Paper no. 77-126.

Würz, W., Herr, S., Wörner, A., Rist, U., Wagner, S., and Kachanov, Y. S., 2003, "Three-Dimensional Acoustic-Roughness Receptivity of a Boundary Layer on an Airfoil: Experiment and Direct Numerical Simulations," J. Fluid Mech., 478, pp. 135-163.

Wyganski, I., 1981, "The Effect of Reynolds Number and Pressure Gradient on the Transitional Spot in a Laminar Boundary Layer," Lecture Notes in Physics: The Role of Coherent Structures in Modelling Turbulence and Mixing, 136, p. 304.

Wyganski, I., Zilberman, M., and Haritonidis, J. H., 1982, "On the Spreading of a Turbulent Spot in the Absence of a Pressure Gradient," J. Fluid Mech., 123, pp. 69-90.

Yang, Z. and Voke, P. R., 2001, "Large-Eddy Simulation of Boundary-Layer Separation and Transition at a Change of Surface Curvature," J. Fluid Mech., 493, pp. 305-333.

Yaras, M. I., 2001, "Measurements of the Effects of Pressure-Gradient History on Separation-Bubble Transition," ASME Paper no. 2001-GT-0193.

Yaras, M. I., 2002, "Measurements of the Effects of Freestream Turbulence on Separation-Bubble Transition," ASME Paper no. GT2002-30232.

Yeung, P. K. and Pope, S. B., 1989, "Lagrangian Statistics from Direct Numerical Simulations of Isotropic Turbulence," J. Fluid Mech., 207, pp. 531-586. 
Zhong, S., Chong, T. P., and Hodson, H. P., 2002, "On the Spreading Angle of Turbulent Spots in Non-Isothermal Boundary Layers with Favourable Pressure Gradients," ASME Paper no. GT2002-30222.

Zhong, S., Kittichaikarn, C., Hodson, H. P., and Ireland, P. T., 2000, "Visualisation of Turbulent Spots under the Influence of Adverse Pressure Gradients," Exp. Fluids, 28, pp. 385-393. 


\section{Appendix A}

\section{Test Section Ceiling Geometry}

In this appendix, the test section ceiling geometry settings are summarized for each of the four pressure distributions. The geometric data are given in Tables A.1 and A.2, which is explained briefly with the aid of Fig. A.1.

In this figure, the adjustment bars are represented by open circles, and are mounted on threaded rods to facilitate adjustment of the ceiling geometry. The angles in Table A.2 are measured by placing a digital protractor on top of the adjustment bars, and are adjusted to within $0.1^{\circ}$. The filled circle at the rear of the test section (left in the figure) is a fixed bar upon which the aft portion of the flexible ceiling rests. Downstream of this fixed bar, the ceiling is maintained in its position by the inflexible ceiling in the downstream portion of the test section. The arrangement of this part of the test section however is not crucial, since it is downstream of the end of the test plate. At the inlet to the test section, the front portion of the flexible ceiling is fixed to the upper front corner of the test section by a hinged connection. The height of the first adjustment bar is set such that the flexible ceiling is horizontal up 


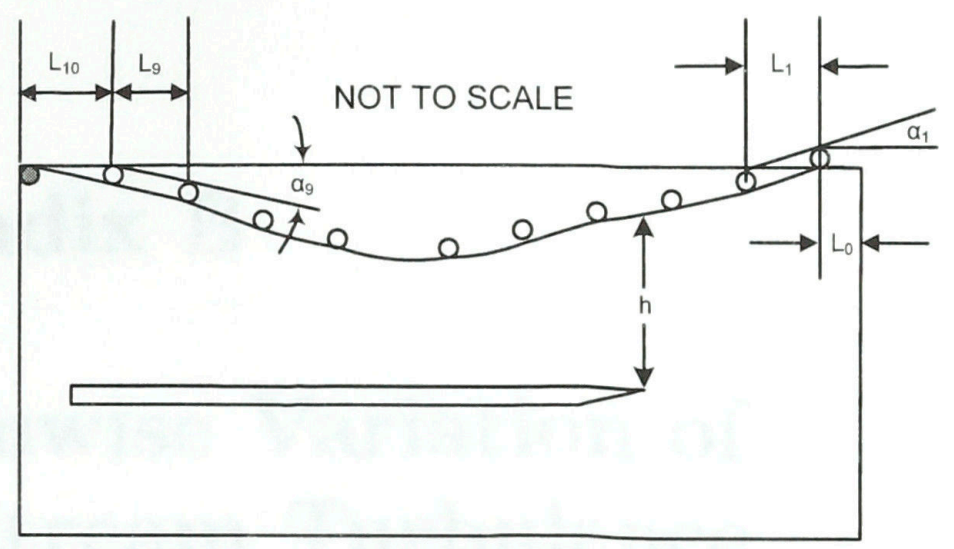

Figure A.1: Schematic of test section ceiling geometry

Table A.1: Test section ceiling geometry adjustment bar spacing

\begin{tabular}{|l|c|c|c|c|c|c|c|c|c|c|c|}
\hline Bar & $L_{0}$ & $L_{1}$ & $L_{2}$ & $L_{3}$ & $L_{4}$ & $L_{5}$ & $L_{6}$ & $L_{7}$ & $L_{8}$ & $L_{9}$ & $L_{10}$ \\
\hline spacing (in.) & 3.0 & 5.5 & 5.5 & 6.0 & 6.0 & 6.0 & 9.0 & 6.0 & 6.0 & 6.0 & 6.25 \\
\hline
\end{tabular}

Table A.2: Test section ceiling geometry settings (angles in degrees)

\begin{tabular}{|l|c|c|c|c|}
\hline $\begin{array}{l}\text { Pressure } \\
\text { distribution }\end{array}$ & $C_{P 1}$ & $C_{P 2}$ & $C_{P 3}$ & $C_{P 4}$ \\
\hline$h(\mathrm{~mm})$ & 169 & 172 & 170 & 169 \\
\hline$\alpha_{0}$ & 0.0 & 0.0 & 0.0 & 0.0 \\
\hline$\alpha_{1}$ & 19.2 & 19.0 & 19.0 & 19.6 \\
\hline$\alpha_{2}$ & 6.0 & 6.0 & 6.0 & 5.7 \\
\hline$\alpha_{3}$ & 0.3 & 1.6 & 0.0 & 0.2 \\
\hline$\alpha_{4}$ & 14.9 & 14.2 & -7.0 & 15.0 \\
\hline$\alpha_{5}$ & 13.1 & 11.7 & -2.5 & 13.4 \\
\hline$\alpha_{6}$ & -12.2 & -10.0 & -2.5 & -7.6 \\
\hline$\alpha_{7}$ & -9.7 & -10.1 & -2.0 & -7.7 \\
\hline$\alpha_{8}$ & -11.7 & -12.0 & -2.0 & -10 \\
\hline$\alpha_{9}$ & -17.5 & -12.0 & -2.0 & -15.3 \\
\hline
\end{tabular}

to this point. For reference, the tip of the test plate leading edge is located $500 \mathrm{~mm}$ downstream of the beginning of the contoured ceiling, between adjustment bars 3 and 4. 


\section{Appendix B}

\section{Streamwise Variation of Free-Stream Turbulence}

The correlations presented in this thesis are based on the free-stream turbulence quantities at the inlet to the test section, because turbulence quantities at transition inception are not often known during the design stages. However, to allow comparison of data from other wind tunnel facilities to the present test section, it is often necessary to compare the variation of free-stream turbulence as the flow develops along the test surface. Therefore, for completeness of the current work, this variation of free-stream turbulence intensity is documented in this appendix. The data are presented in Figs. B.1-B.12 below, for each of the pressure distributions, turbulence grids, and Reynolds numbers for which experiments were conducted in the present study. The surface roughness has no effect on the variation of free-stream turbulence. Therefore, to avoid cluttering the figures, only the data for the smooth surface are presented. 


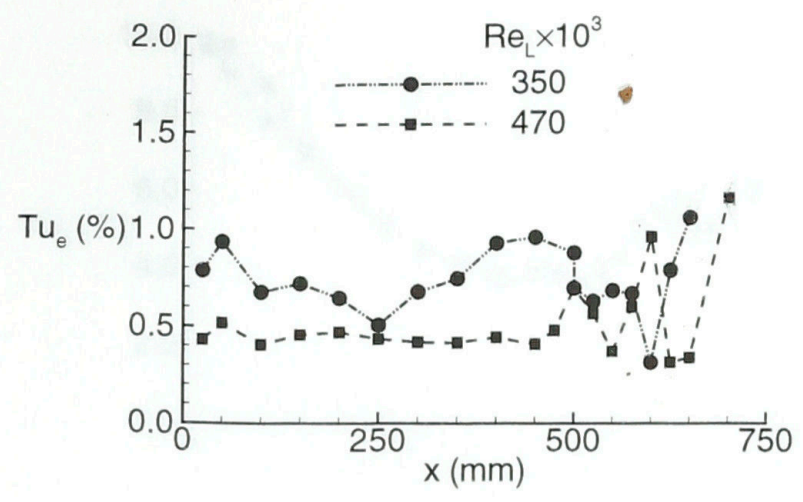

Figure B.1: Variation of free-stream turbulence intensity $C_{P 1}$ pressure distribution; no grid installed

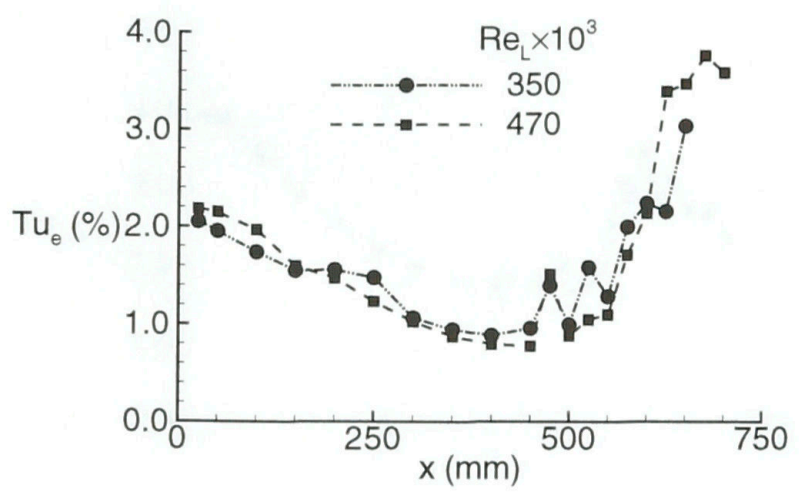

Figure B.2: Variation of free-stream turbulence intensity $C_{P 1}$ pressure distribution; turbulence grid \#1

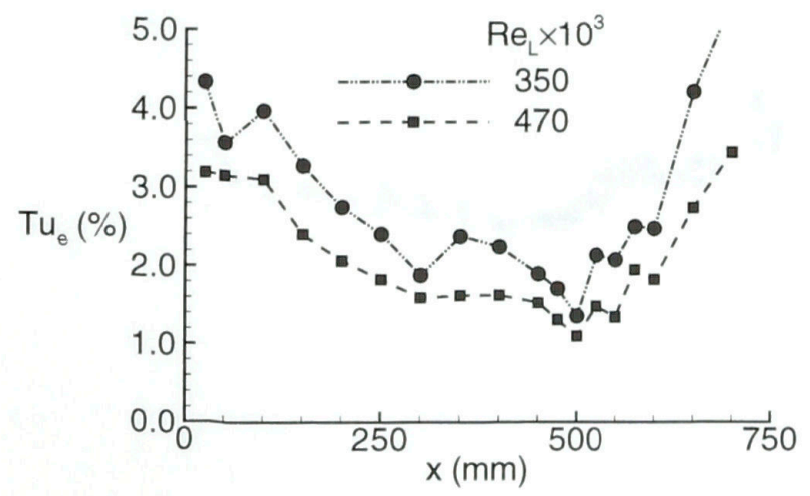

Figure B.3: Variation of free-stream turbulence intensity $C_{P 1}$ pressure distribution; turbulence grid \#2 


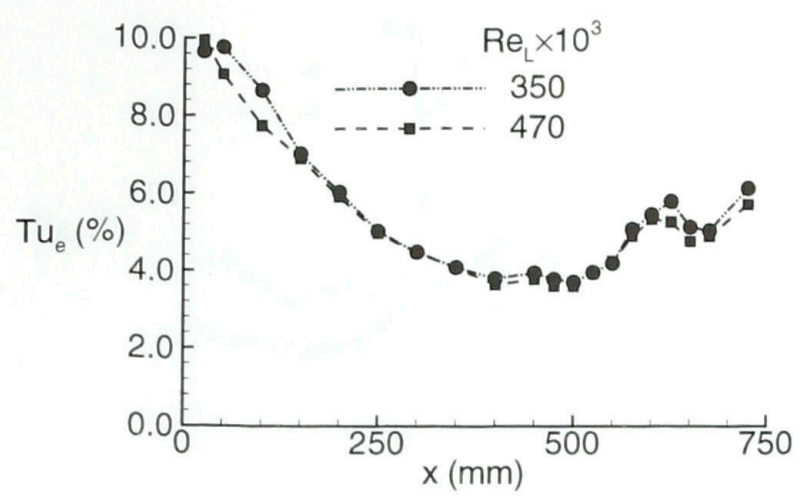

Figure B.4: Variation of free-stream turbulence intensity $C_{P 1}$ pressure distribution; turbulence grid \#4

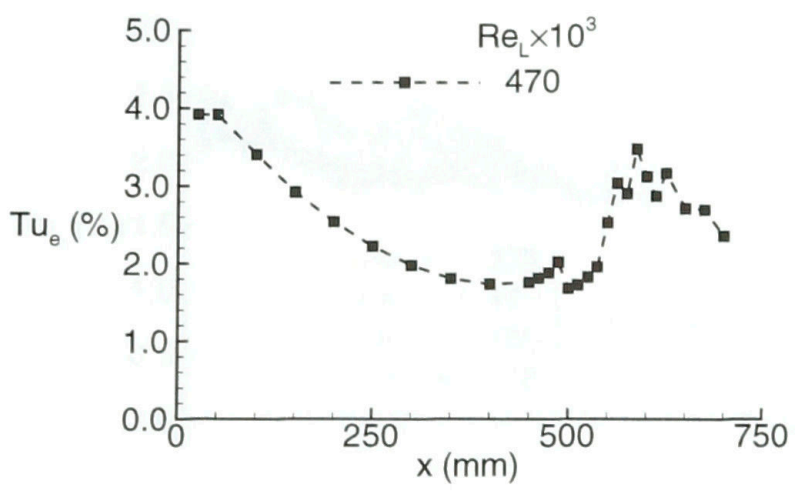

Figure B.5: Variation of free-stream turbulence intensity $C_{P 2}$ pressure distribution; turbulence grid \#4

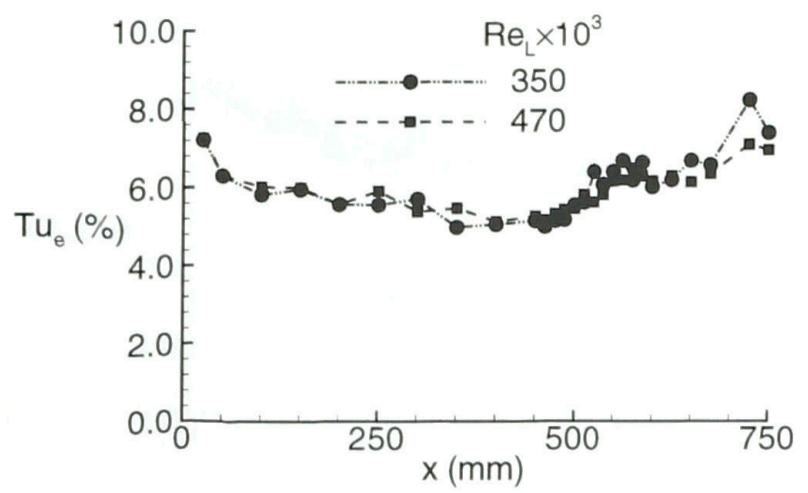

Figure B.6: Variation of free-stream turbulence intensity $C_{P 2}$ pressure distribution; turbulence grid $\# 5$ 


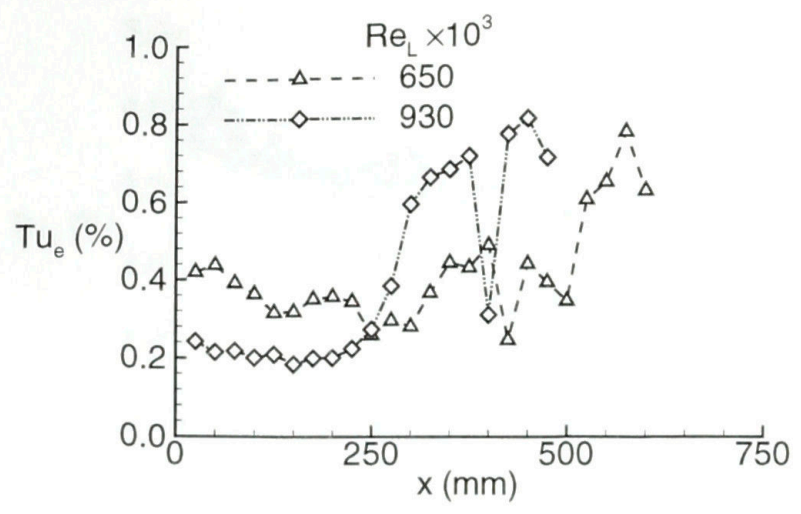

Figure B.7: Variation of free-stream turbulence intensity $C_{P 3}$ pressure distribution; no turbulence grid

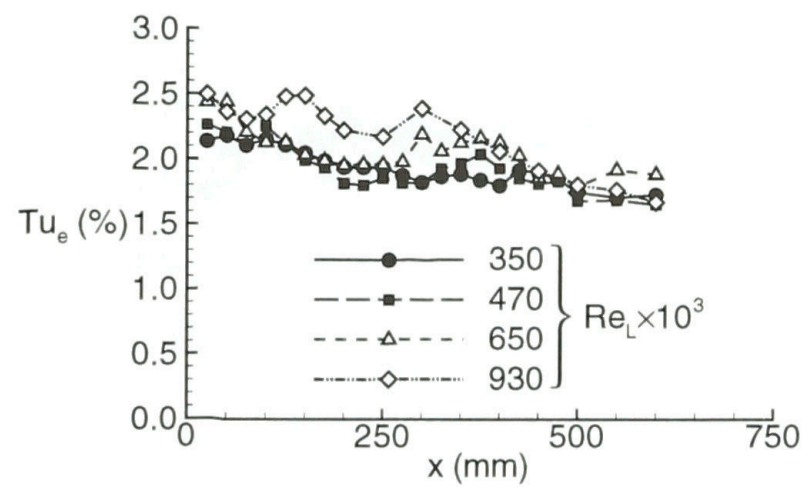

Figure B.8: Variation of free-stream turbulence intensity $C_{P 3}$ pressure distribution; turbulence grid \#1

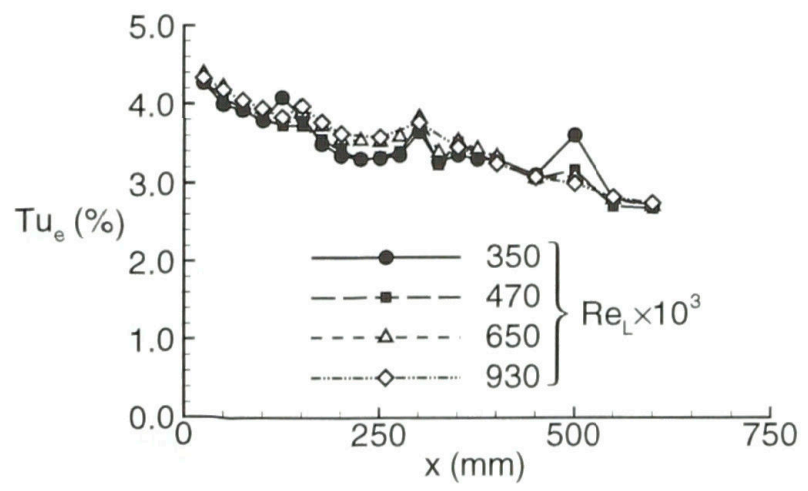

Figure B.9: Variation of free-stream turbulence intensity $C_{P 3}$ pressure distribution; turbulence grid \#2 


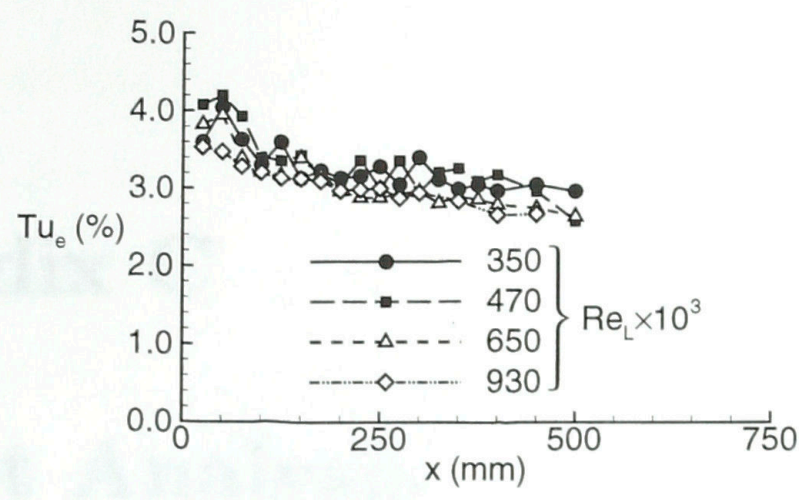

Figure B.10: Variation of free-stream turbulence intensity - $C_{P 3}$ pressure distribution; turbulence grid \#3

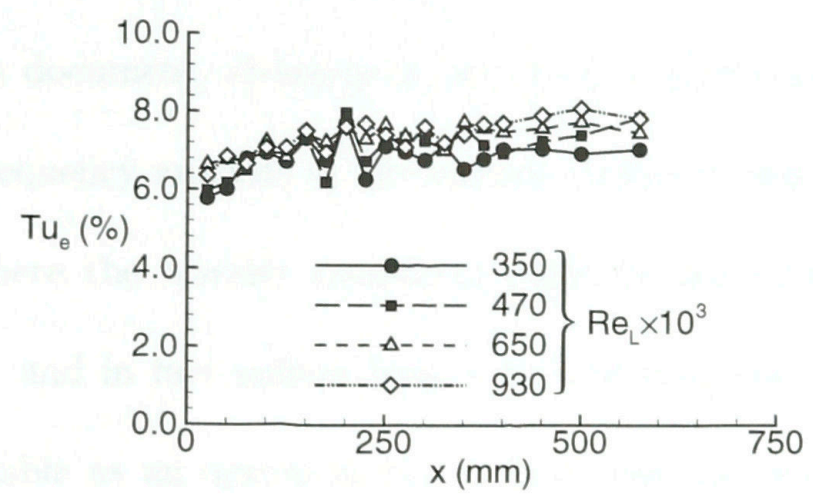

Figure B.11: Variation of free-stream turbulence intensity - $C_{P 3}$ pressure distribution; turbulence grid \#4

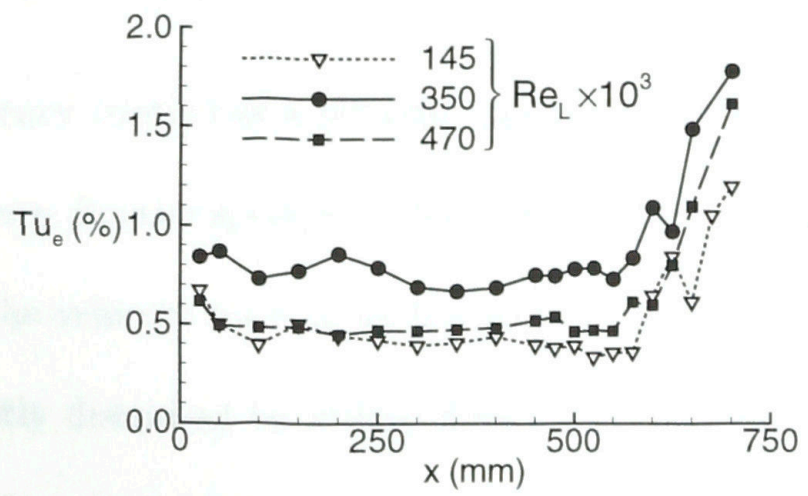

Figure B.12: Variation of free-stream turbulence intensity $-C_{P 4}$ pressure distribution; no grid installed 


\section{Appendix C}

\section{Wavelet Analysis}

The wavelet transform analysis has not been included in the any of the results presented in this document, although it was used in publications derived from this research. The frequency analysis of the current results is limited to pre-transitional disturbances, where the wavelet transform offers no advantage over the standard Fourier analysis, and in fact suffers from a lack of frequency resolution. However, this tool is available as an option in the data processing program provided on the compact disc in the examination copies of this thesis. At some point in the future it may prove useful in the analysis of growth rates of disturbances during the transition process, and it therefore warrants a brief description.

The frequency content of a periodic signal is often analyzed using the Fourier transform. However, Fourier spectra are less applicable to the analysis of non-periodic signals, such as the velocity trace of an intermittently turbulent flow, or a boundary layer intermittently disturbed by wakes shed from upstream components. In these instances, it is often desirable to differentiate the flow into periods of interest, and 
study the frequency content of these subsets of the signal separately. Although this can be achieved through the use of a windowed Fourier transform, the frequency resolution will be poor if the window is of short duration. To overcome the limitations of the Fourier analysis, the wavelet transform is used by a growing number of research groups (e.g. Volino, 2002-a; Schobeiri et al., 1995) in their studies of intermittent flow phenomena. A description of the method used in the present study is presented below.

The primary advantage of the wavelet transform is that, unlike the Fourier transform, it differentiates not only the frequencies present in a signal, but also the times at which these frequencies dominate. This is achieved by correlation of the signal with a test function, or wavelet, which simulates the waveform of the original signal within a localized area. The test function used in the present study is the Marr, or "Mexican hat", wavelet:

$$
g(t)=\sqrt{c}\left((c t)^{2}-1\right) e^{-\frac{(c t)^{2}}{2}}
$$

where $c=2 \pi f \sqrt{2 / 5}$ is the dilation factor for the Marr wavelet that simulates a signal at the frequency $f$. This wavelet function is commonly used in the analysis of fluid flow, since, among other reasons, it provides a good approximation to a single period of a sinusoidal function. By varying the dilation factor $c$, and shifting the position of the wavelet in time, as shown schematically in Fig. C.1, the correlation between the wavelet and the signal can be computed for a number of frequencies, and at different points in time. Mathematically, this is equivalent to computing the 


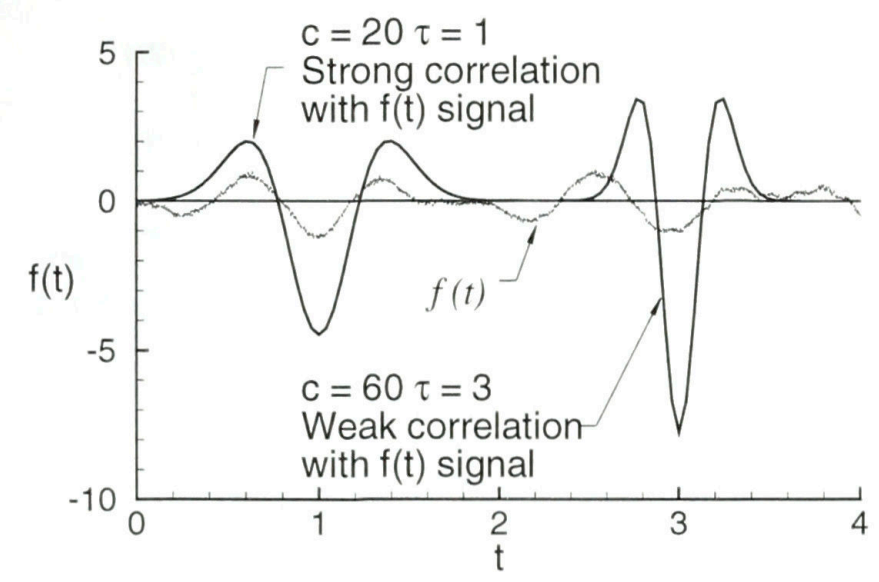

\section{Figure C.1: Schematic representation of the wavelet transform process}

convolution of the wavelet with the signal over a range of frequencies of interest. In the current study, the wavelet transform coefficients are calculated in a discretized time-frequency domain as follows:

$$
h\left(f, t_{i}\right)=\frac{2}{\sqrt{\pi} N} \sum_{j=1}^{N} u^{\prime}\left(t_{j}\right) \times g\left(f, t_{j-1}\right)
$$

In Eq. C.2, the factor $2 / \sqrt{\pi}$ ensures that the power spectrum and total energy calculated on the basis of the wavelet transform of the u' signal (Eqs. C.3 and C.4 below) are consistent with those based on the Fourier transform of $u^{\prime}$ and the energy of the actual signal, respectively.

$$
\begin{gathered}
P S D(f)=\frac{1}{N} \sum_{i=1}^{N}\left(h\left(f, t_{i}\right)\right)^{2} \\
\overline{u^{\prime}}=\sqrt{\sum_{f} P S D(f) \Delta f}
\end{gathered}
$$




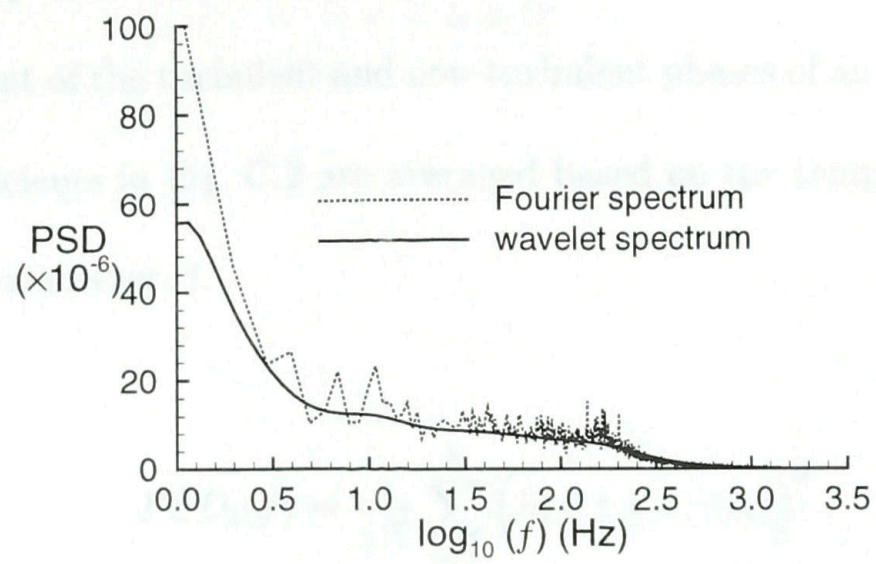

\section{Figure C.2: Comparison of wavelet-averaged and Fourier power spectra}

In the present work, the wavelet transform is computed at 60 discrete frequencies, ranging from $4 \mathrm{~Hz}$ to $4 \mathrm{kHz}$, equally spaced on a logarithmic scale. This range of frequencies has been found to provide adequate frequency resolution, at a reasonable computational expense. The computed energy from Eq. C.4 is typically 2-3\% higher than the energy obtained directly from the time variation of $u^{\prime}$, compared to a negligible difference between the latter and the integrated Fourier spectrum. This implies that computation of the inverse wavelet transform does not result in an exact reproduction of the original signal. However, the good agreement between the power spectrum computed using Eq. C.3 and the corresponding Fourier power spectrum, as demonstrated in Fig. C.2, confirms the validity of the wavelet analysis for the purposes of the present study.

Thus far, the discussion of the wavelet power spectrum has been limited to the time-averaged case. Computation of the power spectrum during a particular event, or series of events, is accomplished by selectively averaging the wavelet transform 
coefficients in Eq. C.2. In the current context, we wish to observe the differences in the frequency content of the turbulent and non-turbulent phases of an intermittent signal. Thus, the coefficients in Eq. C.2 are averaged based on the temporal distribution of the flow state parameter $\beta$.

$$
\begin{gathered}
P S D_{t}(f)=\frac{1}{\gamma N} \sum_{i=1}^{N}\left(h\left(f, t_{i}\right) \times \beta\left(t_{i}\right)\right)^{2} \\
P S D_{l}(f)=\frac{1}{(1-\gamma) N} \sum_{i=1}^{N}\left(h\left(f, t_{i}\right) \times\left(1-\beta\left(t_{i}\right)\right)\right)^{2}
\end{gathered}
$$

The subscripts $t$ and $l$ in Eqs. C.5 \& C.6 denote the turbulent and non-turbulent phases of the flow, respectively. Through the use of this method, frequencies that may dominate during events of short duration are not attenuated by the averaging process inherent in the Fourier transform, and thus further insight into the intermittent behaviour of the flow may be gained. 